\title{
Submillimeter T2 weighted BOLD fMRI of human visual cortex
}

Citation for published version (APA):

Kemper, V. G. (2016). Submillimeter T2 weighted BOLD fMRI of human visual cortex. [Doctoral Thesis, Maastricht University]. Maastricht University. https://doi.org/10.26481/dis.20160421vk

Document status and date:

Published: 01/01/2016

DOI:

10.26481/dis.20160421vk

Document Version:

Publisher's PDF, also known as Version of record

\section{Please check the document version of this publication:}

- A submitted manuscript is the version of the article upon submission and before peer-review. There can be important differences between the submitted version and the official published version of record.

People interested in the research are advised to contact the author for the final version of the publication, or visit the DOI to the publisher's website.

- The final author version and the galley proof are versions of the publication after peer review.

- The final published version features the final layout of the paper including the volume, issue and page numbers.

Link to publication

\footnotetext{
General rights rights.

- You may freely distribute the URL identifying the publication in the public portal. please follow below link for the End User Agreement:

www.umlib.nl/taverne-license

Take down policy

If you believe that this document breaches copyright please contact us at:

repository@maastrichtuniversity.nl

providing details and we will investigate your claim.
}

Copyright and moral rights for the publications made accessible in the public portal are retained by the authors and/or other copyright owners and it is a condition of accessing publications that users recognise and abide by the legal requirements associated with these

- Users may download and print one copy of any publication from the public portal for the purpose of private study or research.

- You may not further distribute the material or use it for any profit-making activity or commercial gain

If the publication is distributed under the terms of Article $25 \mathrm{fa}$ of the Dutch Copyright Act, indicated by the "Taverne" license above, 


\title{
Submillimeter $\mathrm{T}_{2}$ weighted BOLD fMRI of human visual cortex
}

\author{
Valentin G. Kemper
}

(C) Valentin G. Kemper, Maastricht 2016

All rights reserved. No part of this thesis may be reproduced, stored in a retrieval system, or transmitted in any form, or by any means, electronic, mechanical, photocopying, recording, or otherwise, without prior written permission of the author.

Printed by CPI-Koniklijke Wöhrmann - Zutphen

ISBN: 978-94-6328-029-7 


\section{Submillimeter $\mathrm{T}_{2}$ weighted BOLD fMRI of human visual cortex}

\section{Dissertation}

to obtain the degree of Doctor at Maastricht University, on the authority of Rector Magnificus, Prof.dr. L.L.G. Soete, in accordance with the decision of the Board of Deans, to be defended in public on Thursday, April 21, 2016, at 16:00 hours by Valentin Gereon Kemper 
Supervisor:

Prof.dr. Rainer Goebel

Co-supervisors:

Prof.dr. Essa Yacoub, University of Minnesota

Dr. Federico De Martino

Assessment Committee:

Prof.dr. Elia Formisano (Chairman) Dr. Jonathan R. Polimeni, Harvard University Prof.dr. Kâmil Uğurbil, University of Minnesota

Dr. Kâmil Uludağ

The work presented in this thesis was funded by the European Research Council (ERC grant number 269853). 



\section{Table of contents}

Table of contents 5

$\begin{array}{ll}\text { Abbreviations, acronyms, variables } & 7\end{array}$

Chapter 1 Introduction 9

Chapter 2 Submillimeter $\mathrm{T}_{2}$ weighted $\mathrm{fMRI}$ at $7 \mathrm{~T}$ : comparison of 3D-GRASE and 2D SE-EPI 51

Chapter 3 Variable flip angle 3D-GRASE for high resolution $\mathrm{fMRI}$ at $7 \mathrm{~T}$

Chapter $4 \quad$ 9.4 T anatomical and functional investigation of human primary and secondary visual cortex

Chapter 5 Summary and discussion

Knowledge Valorization 160

Acknowledgements 165

Curriculum Vitae 166

$\begin{array}{ll}\text { Publication List } & 167\end{array}$

$\begin{array}{ll}\text { References } & 170\end{array}$ 



\section{Abbreviations, acronyms, variables}

$\begin{array}{ll}\mathrm{B}_{0} & \text { Static magnetic field } \\ \mathrm{B}_{1}{ }^{+/} & \text {Transmission (+)/receive (-) radio frequency magnetic field } \\ \text { BOLD } & \text { Blood oxygen level dependent signal } \\ \text { CBF } & \text { cerebral blood flow } \\ \text { CBV } & \text { cerebral blood volume } \\ \text { CMRO2 } & \text { cerebral metabolic rate of oxygen } \\ \text { CNR } & \text { Contrast-to-noise ratio } \\ \text { CSF } & \text { Cerebrospinal fluid } \\ \text { EPI } & \text { Echo planar imaging } \\ \text { fMRI } & \text { functional magnetic resonance imaging } \\ \text { FoV } & \text { Field of View } \\ \text { FSE } & \text { Fast spin echo (RARE equivalent) } \\ \text { FWHM } & \text { Full width at half maximum } \\ \text { GE-/SE- } & \text { Gradient-echo / spin-echo } \\ \text { GLM } & \text { General linear model } \\ \text { GRASE } & \text { gradient and spin-echo } \\ \text { GRE } & \text { Gradient (recalled) echo } \\ \text { HASTE } & \text { Half Fourier acquisition single-shot turbo spin-echo } \\ \text { hMT+ } & \text { human homologue of middle temporal complex (also V5) } \\ \text { LGN } & \text { lateral geniculate nucleus } \\ \text { MB } & \text { Multiband } \\ \text { MION } & \text { monocrystalline iron oxide nanoparticles } \\ \text { MPRAGE } & \text { Magnetization-prepared rapid gradient-echo } \\ \text { MRI } & \text { Magnetic resonance imaging } \\ \text { MTF } & \text { Modulation transfer function } \\ \text { NMR } & \text { nuclear magnetic resonance } \\ \text { ODC } & \text { ocular dominance column } \\ \text { PET } & \text { positron emission tomography } \\ \text { RARE } & \text { Rapid acquisition with relaxation enhancement } \\ \text { RF } & \text { Radiofrequency } \\ \text { ROI } & \text { Region of interest } \\ \text { PSF } & \text { Point-spread function } \\ \text { SAR } & \text { Specific absorption rate } \\ \text { SMS } & \text { Simultaneous multi slice } \\ \text { SNR } & \text { Signal-to-noise ratio } \\ \text { (b)SSFP } & \text { (balanced) steady-state free precession } \\ \mathrm{T}_{1} & \text { Longitudinal relaxation time } \\ \mathrm{T}_{2}{ }_{2}{ }^{*} & \text { Transversal relaxation time } \\ \text { TE } & \text { Effective transversal relaxation time } \\ \text { TI } & \text { Echo time } \\ & \text { Inversion time } \\ & \end{array}$


Repetition time

TSE

Turbo spin echo (RARE equivalent)

tSNR

temporal signal-to-noise ratio

V1

primary visual cortex

V2 secondary visual cortex

VASO

Vascular space occupancy

VFA variable flip angle 
Chapter 1

Introduction 
Magnetic resonance imaging (MRI) employs a combination of static and fast alternating magnetic fields to obtain information about the inner structure of the studied object. Not only is MRI a mainstay in medical imaging, it can also be used to trace neuronal activity in the brain indirectly by detecting dynamic signal changes associated with changes in blood oxygenation, which are induced by the changed metabolic demand of the involved neurons. Over the past two decades, this fascinating technique called functional MRI (fMRI) has developed to become one of the most important methods in cognitive neuroscience, and $\mathrm{fMRI}$ experiments have yielded extensive insight to the functional organization of the living brain. Most fMRI experiments are conducted at temporal resolution of two to three seconds and spatial resolution of several tens of microliters to assess which brain regions are involved in a given task and to what extent. However, key mechanisms of cognitive processes are organized at a finer spatial scale, e.g. the inner layout of a brain region tangentially to the cortical sheet or the laminarization across cortical depth. Investigation of such fine-grained functional structures requires advanced techniques. This thesis deals with such high spatial resolution fMRI techniques. For the successful application in neuroscientific experiments, a solid foundation is required of both the methodological background, and the subject of study, i.e. functional organization at the scale of cortical layers and columns (mesoscale). Therefore, in the following introduction we set out to outline both appropriately. First, the milestones of the development of modern magnetic resonance imaging will be highlighted. Then an account of blood oxygenation level dependent $\mathrm{fMRI}$ and neurovascular coupling is given. Advantages of $T_{2}$ weighted BOLD fMRI follow. A brief review of the architecture of cortical gray matter at the scale of layers and columns is given with special interest in early visual cortex, and particularly primary visual cortex (V1). Then, aspects of prime relevance for high resolution imaging are considered. The point spread function of echo-train sequences is described mathematically, and finally, the 3D-GRASE sequence will be described briefly. An overview of the following chapters is given at the end of this introduction.

\section{Beginnings of Magnetic Resonance Imaging}

An electric current causes a magnetic field. This classical electro-magnetic observation is paralleled in quantum mechanics: charged elementary 
particles like electrons or the constituents of a proton possess a magnetic field caused by a constant rotation about its own axis, its spin. The fact that atomic nuclei possess such a magnetic moment was first described by Isidor Isaac Rabi in 1938, who thereby extended the findings of Otto Stern and Walther Gerlach on the quantization of electron spins. Rabi used molecular beams traversing homogeneous and opposing inhomogeneous magnetic fields. Under the influence of radiofrequency (RF) waves at specific frequencies, the trajectory of the beam was altered, because the magnetic state of the atoms had been manipulated by the RF field. More specifically, if the Larmor frequency ${ }^{1}$ of the nuclei in the beam were matched by the transmitted RF field, transitions of energetic levels associated with the orientation of the nuclear spin moment with regard to the external magnetic field were facilitated, and the altered magnetic configuration with respect to the external magnetic field caused deflection of the beam. Felix Bloch and independently Edward Mills Purcell experimented later, in 1946, with nuclear magnetic resonance (NMR) in solids and liquids. Bloch also provided great parts of the theoretical description of the precession of the nuclear magnetic moments in the external magnetic field. Although in comparison to nuclear beams, the separation of magnetic states is much weaker in solids, the abundance of atoms in solids compared to molecular beams allowed studying the resonance effects with more simple methods. It should be noted that the so-called Zeeman splitting (the difference of energetic states of magnetic levels) of protons at room temperature and 1 Tesla field strength is only $1.8 \times 10^{-7}$ electron volts ${ }^{2}$, and that as a consequence of the Fermi-Dirac distribution, the higher energetic state is only approximately $10^{-6}$ less occupied than the lower state. In other words, the observable magnetization stems from only one millionth of the total nuclear magnetic moment. The original saturation method was improved by the application of pulsed methods, to generate both free induction decays (Hahn, 1950a) and spin-echoes (Hahn, 1950b). This way, NMR spectroscopy could reveal information about the compounds of solids and their chemical environment, e.g. in which molecules hydrogen is bound.

1 Named after Joseph Lamor (1857-1942). The frequency at which a magnetic dipole precesses in an external magnetic field.

21 Electron volt (eV) equals $1.602 \times 10^{-19}$ Joule. 
The usage of static field gradients for spatial encoding of the signal source was introduced by Paul Lauterbur. This way, a projection of the object spin density onto the axis of the gradient could be obtained (Lauterbur, 1973; 1974). Peter Mansfield identified the possibility to sample multiple echoes after a single excitation (Mansfield, 1977), which laid the foundation for echo planar imaging (EPI). This technique could significantly increase the acquisition speed. Additional important contributions to modern (nuclear) magnetic resonance imaging were provided by Richard R. Ernst and colleagues (Kumar et al., 1975), and Donald Twieg (Twieg, 1983). They made more explicit that in MRI one could acquire different spatial frequencies of the object in its dual space, k-space. The relevance of NMR and MRI to life sciences was showcased in the fact that the longitudinal relaxation time $\left(T_{1}\right)$ and transverse relaxation time $\left(T_{2}\right)$ differ between tissue types (Damadian, 1971), and further in what can be considered the first human body MRI image (Damadian et al., 1977). In the following years many improvements and developments in the domains of hardware, theoretical concepts, and imaging pulse sequences made MRI more accessible to neuroscientists and clinicians.

\section{The BOLD effect and neuro-vascular coupling}

Functional MRI (fMRI) started in the early 1990's. Functional imaging of the human brain was demonstrated using the external susceptibility contrast agent gadolinium-diethylenetriamine pentaacetic acid (Gd-DTPA, Belliveau et al., 1991). Meanwhile, it could be shown, that also deoxyhemoglobin could serve as a natural, endogenous contrast agent (Ogawa et al., 1990). Hemoglobin in red blood cells contains iron atoms, which lead to an overall diamagnetic behavior when oxygenated (oxyhemoglobin), and to paramagnetic behavior without the oxygen (deoxyhemoglobin). The paramagnetic state causes faster loss of phase-coherence of the magnetization in the vicinity of the molecules, i.e. shorter effective transverse relaxation time, $\mathrm{T}_{2}{ }^{*}$, and hence reduced signal intensity in $\mathrm{T}_{2}{ }^{*}$ weighted images. In other words, the oxygen concentration itself in blood vessels yields a differential signal and can be used to register the location of neuronal activity in the brain. Three groups were in close competition to publish the first experimental human $\mathrm{fMRI}$ results using the newly established contrast mechanism (Bandettini et al., 1992; Kwong et al., 1992; Ogawa et al., 1992), followed by Blamire et al. (1992), Frahm et 
al. (1992), and Turner et al. (1993). The name for the new method given by Ogawa (Ogawa et al., 1990) stuck: Blood oxygenation level dependent contrast, BOLD contrast. Ogawa et al. (1992) employed a 4 Tesla scanner and an unspoiled gradient-echo imaging sequence (FISP), but most sites quickly adapted to using EPI (at any field strength). These first experiments used visual ("photic") stimulation or finger tapping for motor cortex activation in block designs consisting of on/off periods of multiple tens of seconds duration. Several reasons quickly motivated more research groups to study the cerebral functional organization using fMRI. Above all, BOLD imaging is non-invasive and allows application in healthy human volunteers and in patients. Measurements can be repeated virtually infinitely without harming the subject. Also, $\mathrm{fMRI}$ is not limited to the cortical surface or areas which can be easily exposed in animal models for optical imaging. This makes other brain areas accessible to the technique.

An important prerequisite for the interpretation of the BOLD effect is the understanding of vascular coupling to neuronal activity, i.e. where and how the blood oxygenation changes due to neuronal activity. The close spatial link of neuronal activity and increased provision of oxygen and metabolites had been hypothesized for over one-hundred years at that time then (Roy and Sherrington, 1890), and refined over the preceding decades (e.g. Roland and Larsen, 1976; Roland et al., 1980). At the time, it was evident from positron emission tomography (PET) studies that this spatial correspondence was given at least to the scale of cortical areas. In animals, this correspondence was already used at an even finer scale in optical imaging (Grinvald et al., 1986). More recent evidence shows the control of blood flow also at the capillary level (Duong et al., 2001; Attwell et al., 2010; Hall et al., 2014).

At the site of neuronal activity, the metabolic demand increases and more oxygen is extracted from the blood, i.e. the cerebral metabolic rate of oxygen $\left(\mathrm{CMRO}_{2}\right)$ is increased. Although the shorter $\mathrm{T}_{2}{ }^{*}$ of deoxygenated blood should lead to signal reduction, an increase in the order of a few percent is observed. The reason is that the BOLD effect is the result of several mechanisms happening in close temporal relation: The increase of $\mathrm{CMRO}_{2}$ is accompanied by an increase of cerebral blood flow (CBF) and cerebral blood volume (CBV). Both are triggered by a complex cascade of biochemical events (Uludag et al., 2005), which is still not fully understood. Increased CBV also attenuates the signal because of the shorter $\mathrm{T}_{2}{ }^{*}$ of 
blood than in brain parenchyma. Increased CBF increases the signal, because deoxygenated blood is exchanged for oxygen-rich blood. The exact timing and amplitude changes relative to their baselines determine the resultant dynamic signal evolution. All three aspects can be assembled in the hemodynamic response function (Boynton et al., 1996; Friston et al., 1998). The main concomitant BOLD response is positive because the increase in CBF over-compensates the increase in both $\mathrm{CMRO}_{2}$ and $\mathrm{CBV}$ (Fox and Raichle, 1986). The "initial dip", a small negative signal change immediately follows neuronal activity. Although it is not observed everywhere, and the exact underlying mechanism are not perfectly clear (Grinvald et al., 1991; Ernst and Hennig, 1994; Buxton, 2001; Uludag, 2010), it seems that the initial dip signal yields better spatial specificity than the positive response (Duong et al., 2000; Yacoub et al., 2001; but see also Hu and Yacoub, 2012; Watanabe et al., 2013; Siero et al., 2015). When increased $\mathrm{CMRO}_{2}$ and $\mathrm{CBF}$ have decayed back to baseline, the overall $\mathrm{CBV}$ is still above its baseline resulting in a so-called post-stimulus undershoot (Buxton et al., 1998). Again, the exact physiological mechanisms of the post-stimulus undershoot are not clear, and contributions of a CBF undershoot or elevated transient $\mathrm{CMRO}_{2}$ (Donahue et al., 2009) are discussed (Buxton, 2012). However, the post-stimulus undershoot also holds the potential to yield higher spatial specificity than the positive response (Yacoub et al., 2006). An important distinction in this context must be made between the origin of the BOLD signal, which can be extravascular (meaning stemming from the surrounding tissue of a blood vessel) or intravascular. The BOLD response is generated in both compartments, but their temporal dynamics and implications for specificity differ (Yacoub et al., 2006; Bianciardi et al., 2011). Additionally, a distinction between arterial and venous signals is being made, and the main BOLD response is attributed to venous signals (intra- and extravascular).

\section{Higher functional specificity by high-field spin-echo BOLD fMRI}

Shortly after the discovery of the BOLD effect it was found that BOLD imaging could also be done employing $T_{2}$ weighted spin-echo sequences (Bandettini et al., 1994). At first, this finding is counterintuitive because the magnetization near paramagnetic deoxyhemoglobin dephases more quickly than without its presence. In spin-echo imaging, this very dephasing 
effect is reversed by the refocusing RF pulse and when the echo forms, the magnetization is in phase again. However, this is only true for static spins. The water molecules in the brain constantly fulfill a diffusing motion. Hence, an individual spin experiences different static field strengths in the presence of a field gradient caused by deoxyhemoglobin. While the macroscopic dephasing is reversed by the spin-echo, the dephasing due to this diffusion motion remains. The source of the field inhomogeneity has to be sufficiently small so that a diffusing spin can sample enough field variations along its trajectory in the time between excitation and readout. Only then can the $T_{2}$ weighted BOLD effect take place. Importantly, the critical spatial scale is in the order of a few micrometers, the size of cortical capillaries, arteriols and venules (Duvernoy et al., 1981), rendering the extravascular $T_{2}$ weighed BOLD effect sensitive to these blood vessels, but to a lesser extent to larger ones. This difference in sensitivity to small and large vessels increases with increasing field strength. The intravascular $T_{2}$ weighted BOLD contributes the majority of the overall signal at lower field, whereas this contribution is diminished at higher fields because of the short $T_{2}$ of blood itself compared to tissue $T_{2}$ (Boxerman et al., 1995a; Boxerman et al., 1995b; Duong et al., 2003; Uludag et al., 2009)3. In $\mathrm{T}_{2}{ }^{*}$ weighted BOLD imaging, the extravascular signal from both large and small vessels increases with field strength. Therefore, $\mathrm{T}_{2}{ }^{*}$ weighted BOLD imaging is not spatially more specific at high field than at lower field strength (Uludag et al., 2009; Geißler et al., 2013) despite the early optimistic view (Gati et al., 1997). In summary, $T_{2}$ weighted BOLD at high field yields a better spatial specificity than $\mathrm{T}_{2}{ }^{*}$ weighted BOLD (at any field strength) (Yacoub et al., 2005).

The difference in spatio-temporal signal spread between $T_{2}$ and $\mathrm{T}_{2}{ }^{*}$ weighted BOLD can be parametrized by their functional point-spread functions. Besides numerical simulation studies, two main groups of experimental designs to assess functional point spread of a given imaging sequence may be distinguished. In one group, experiments followed the strategy of using response patterns from functional paradigms with spatially well-known functional (e.g. retinotopic) organizations. By contrasting two conditions with neighboring cortical responses,

\footnotetext{
${ }^{3}$ Assuming that the echo time is equal to tissue $T_{2}$.
} 
conclusions about the spread of information can be drawn ${ }^{4}$. It could be shown that at $7 \mathrm{~T}$, the $\mathrm{T}_{2}{ }^{*}$ weighted BOLD contrast has a spread of about $2 \mathrm{~mm}$ (full width at half maximum) and that the spread of $T_{2}$ weighted BOLD is narrower (Engel et al., 1997; Parkes et al., 2005; Shmuel et al., 2007; Panchuelo et al., 2015). In cat visual cortex at 9.4 T, $T_{2}$ weighted BOLD point spread was found to be about $1.0 \mathrm{~mm}$ (Park et al., 2005). This type of experiments does not necessarily require submillimeter resolution. However, the applicability of the results to high resolution $\mathrm{fMRI}$ is somewhat problematic, because an implicit assumption in the model is an isotropic, shift-invariant hemodynamic response. At high spatial resolution the details of the vascular organization (e.g. distribution across the cortical depth or varying density of intracortical veins) contravene this assumption, which may have a strong effect on the specificity (Norris, 2012). Hence, another group of experiments assesses the specificity by investigating laminar differences in activation patterns. It is well known (see below) that cortical area $\mathrm{V} 1$ receives most of its input in layer IV, and hence, most activation is expected there rather than at the cortical surface for a strong bottom-up visual stimulus. Experiments have shown that $T_{2}$ weighted BOLD imaging reflects this behavior more closely than $\mathrm{T}_{2}{ }^{*}$ weighted BOLD (Zhao et al., 2004; Goense and Logothetis, 2006b; Harel et al., 2006; De Martino et al., 2013). Given the anisotropic differences in vascularization tangential or radial to the cortex, and moreover also depth-dependent differences, Polimeni et al. (2010) suggested distinguishing also directional (tangential and radial), and positional (depth-dependent) aspects of the BOLD pointspread function (see also Discussion chapter).

It must be mentioned that also other, more direct measures exist to assess the components of the BOLD effect individually for high resolution fMRI. Specifically, it could be shown that CBF (Zappe et al., 2008; Goense et al., 2012) and CBV (Harel et al., 2006; Jin and Kim, 2008a; Jin and Kim, 2008b; Goense et al., 2012) yielded cortical activity depth profiles more in line with the expectation described above. These CBV experiments performed on animals employed monocrystalline iron oxide nanoparticles (MION) to increase the CBV contrast. This is not possible in the human. However, the vascular space occupancy dependent (VASO, Lu et al., 2003) technique has

\footnotetext{
${ }^{4}$ In the model, neuronal spread by lateral excitatory or inhibitory connections is ignored, and the spread is attributed solely to vasculature.
} 
recently been developed further for application in humans without exogenous contrast agents (Hua et al., 2013a; Huber et al., 2013; Huber et al., 2015a).

The functional point-spread function takes signal spread into account which is attributed to the vascularization and the contrast mechanisms of the imaging sequence. The signal spread due to the imaging process itself is ignored in numerical simulations of the functional point spread function and only implicitly accounted for in experiments (because it is unavoidable). It must, however, be considered because different contrast mechanisms require the use of different imaging sequences, and the imaging point spread functions may differ. The explicit formulation of the imaging point spread function follows further below.

\section{Mesoscopic organization of the cerebral cortex}

The structural and functional organization of the human cerebral cortex can be broadly divided into three spatial scales: Macroscopic, mesoscopic and microscopic. In-vivo, the macro- and mesoscale are nowadays accessible by MRI approaches, but the microscale remains the domain of other methods (although microscopic composition can be inferred from MR data, e.g. spectroscopic, relaxometric or diffusion data). At the macroscale, one distinguishes four different lobes (frontal, temporal, occipital, and parietal) in the two hemispheres, which accommodate multiple cortical gray matter areas. These can be distinguished based on their microscopic properties. First systematic accounts of this organization were given by the study of post-mortem specimen (Brodmann, 1909), in which different neurons and neuronal cell densities as well as different connectivity patterns were observed. Brodmann distinguished about 52 different areas across the brain by the means of histological staining techniques (Ramón y Cajal, 1899), which were based on the method of Camillo Golgi (1843-1926). To the contemporaries, it was already evident that the different anatomical structures must also serve different functional, behavioral purposes. Such functional localization in different brain areas could also be concluded from lesion case studies, e.g. (Broca, 1865; Harlow, 1868; Wernicke, 1874; Damasio et al., 1994). Since these early days, a multitude of methods including lesion studies, but also fMRI, has detailed the knowledge of functional specialization, and many areas with involvement in specific tasks could be identified. Prominent examples 
from the visual domain include visual areas V1-V4, V5/hMT+, the fusiform face area (Kanwisher et al., 1997), the extrastriate body area (Downing et al., 2001), and the parahippocampal place area (Epstein and Kanwisher, 1998). To what extent macroanatomical folding patterns are respected by the functional areas, and whether their boundaries are strictly linked to microanatomical differences is still topic of current research (Zilles and Amunts, 2009; Wilms et al., 2010; Frost and Goebel, 2012). Across the brain, maps can be found which are large enough to be considered also macroscopic: Retinotopy in visual areas (Engel et al., 1994; DeYoe et al., 1996; Wandell et al., 2007; Silver and Kastner, 2009), tonotopy in auditory cortex (Formisano et al., 2003), (gross) somatotopy in motor areas and somatosensory areas (Lotze et al., 2000; Buccino et al., 2001), while finer bodily differences (e.g. single digits, Besle et al., 2014) also have more finegrained representations.

The mesoscale of the cerebral cortex can best be understood when mentally unfolding the macroscopic gyri and sulci to obtain a flat sheet of the thickness of the cortical gray matter. Perpendicular to this sheet, along the cortical depth, a generic model of six cortical layers has proven useful (Brodmann, 1909). These are numbered from the outer (pial) surface increasing towards the inside, towards the white matter. In various brain areas, the neuronal organization within the layers and the intracortical connectivity between layers is similar (Vogt and Vogt, 1919; Nieuwenhuys, 2013). In a simplified model, feed-forward connections from earlier stages of processing, e.g. visual information entering the cortex in primary visual cortex, V1, from the lateral geniculate nucleus (LGN, a pulvinar thalamic nucleus, which relays the information from the retina of the eye), terminate mostly in distinct sublayers of cortical layer 4. Within V1, the information transverses across the layers, and feed-forward connections to higher areas start from cortical layers 2, 3, 5 and 6. Feedback connections from higher areas terminate in layers 2, 3 and 5 . The full connectivity is more complex (Gilbert, 1983; Douglas and Martin, 1991; Felleman and Van Essen, 1991; Douglas and Martin, 2004; Sincich and Horton, 2005).

fMRI studies probing human cortical depth dependent activation have become possible in recent years e.g. (Ress et al., 2007; Koopmans et al., 2010; Polimeni et al., 2010; Koopmans et al., 2011; Zimmermann et al., 2011; Olman et al., 2012; De Martino et al., 2013; Huber et al., 2015a). In the light of the extensive knowledge about laminar structure from other 
methods, their impact to neuroscience is to date rather limited. Rather, thus far, they are informative about possibilities and limitations of the method itself. Applications mostly focused on visually evoked response profiles across cortical depth. Nevertheless, the potential of cortical depth dependent $\mathrm{fMRI}$ studies is exemplified in studies targeting different signal pathways such as magno- and parvocellular pathways (Olman et al., 2012), or feedforward or feedback connections (Muckli et al., 2015).

Within brain areas, tuning of the neurons parallel to the cortical sheet is not uniform, nor are neurons with similar tuning properties scattered across the area at random. Instead, a more fine-grained organization exists. Based on micro-electrode recordings in cat somatosensory cortex, Mountcastle (1957) postulated the presence of elementary processing units with highly similar tuning properties, which span the entire cortical depth forming a cortical column. This pioneering work was followed up by Hubel and Wiesel, who found also a columnar organization in visual cortex of cats and primates (Hubel and Wiesel, 1962; 1965; 1968; 1974) using also micro-electrode recordings, lesion studies in the LGN, or the injection of $[3 \mathrm{H}]$ proline into one eye (Wiesel et al., 1974). Across these studies, the insight emerged that the primary visual cortex consists of domains of eye preferences, termed ocular dominance columns (ODCs). Using autoradiography, the complete pattern of ocular dominance columns could be presented in the macaque monkey (LeVay et al., 1985). These patches resemble zebra-stripes and are of several hundreds of micrometers in thickness. In the following years, multiple additional tuning characteristics of V1 neurons were observed: Preference for stimulus orientation, direction of motion, spatial and temporal frequency (Hubel and Wiesel, 1963; Bonhoeffer and Grinvald, 1991; Blasdel, 1992; Weliky et al., 1996; Shoham et al., 1997). These are closely linked to specific parallel thalamocortical and cortico-cortical connections to, from, and within V1, and their distinct projection zones in V2 (see (Sincich and Horton, 2005; Shapley and Hawken, 2011) for review).

In the human brain, ODCs have been shown in post-mortem samples using cytochrome oxidase staining in monocular blind (Horton and HedleyWhyte, 1984; Adams et al., 2007), after it had been validated that cytochrome oxidase staining and $[3 \mathrm{H}]$ proline injections yielded the same ODC patterns in animals (Horton, 1984). Using fMRI, ODCs could be visualized also in-vivo in the human brain (Menon et al., 1997; Dechent and 
Frahm, 2000; Cheng et al., 2001; Goodyear and Menon, 2001; Yacoub et al., 2007). With the exceptions of orientation columns in V1 (Yacoub et al., 2008), temporal frequency (Sun et al., 2007) and axis-of-motion selective columns in hMT/V5 (Zimmermann et al., 2011; De Martino et al., 2013), no further columnar structures could be reliably mapped using fMRI in humans thus far (but informational content could be inferred, see Discussion chapter). This circumstance likely reflects conceptual controversies (Horton and Adams, 2005; Adams and Horton, 2009; Ts'o et al., 2009; da Costa and Martin, 2010), but more importantly technical and methodological limitations.

\section{Imaging considerations for high-resolution fMRI}

BOLD imaging is perfectly suitable to study cognitive processes in the human brain at the temporal scale of seconds and spatial scale of several cubic millimeters, probing of the involvement of macroscopic brain areas in a given task. Coverage of the entire brain is feasible at increasingly higher temporal and spatial resolutions thanks to various fast (Feinberg et al., 1986; Frahm et al., 1986; Margosian et al., 1986) and ultra-fast imaging techniques (Mansfield, 1977; Poser et al., 2010) and accelerated imaging techniques (Pruessmann et al., 1999; Griswold et al., 2002; Breuer et al., 2006). Most recently, the development of simultaneous multi-slice (SMS, also multiband, $\mathrm{MB}$ ) imaging has significantly increased imaging speed and/or spatial resolution further (Larkman et al., 2001; Feinberg et al., 2010; Moeller et al., 2010; Setsompop et al., 2012). This development was made possible and further nourished by the introduction of multiple receive $\mathrm{RF}$ coil elements with spatially independent receive profiles (Sodickson and Manning, 1997; Wiggins et al., 2009). When aiming for high spatial resolution, special care must be taken to maintain sufficient sensitivity to identify functionally induced signal changes. Some means for higher sensitivity are the same as at lower resolution, but others differ markedly.

For high sensitivity, the contrast-to-noise ratio (CNR) has to be high. CNR is proportional to the effect size (i.e. BOLD signal change) and the temporal signal-to-noise ratio (tSNR). tSNR, in turn, scales with the signal-to-noise ratio (SNR) of the individual images in a time series. At low resolution, physiological noise dominates the tSNR. That is, signal fluctuations induced by physiological processes such as respiration and cardiac pulsation limit 
the tSNR more strongly than the thermal noise affecting the acquisition (Triantafyllou et al., 2005; Triantafyllou et al., 2011). A considerable number of acquisition techniques (Zhang et al., 2006; Kundu et al., 2012) and post-processing techniques (Hu et al., 1995; Glover et al., 2000; Birn et al., 2008; Chang and Glover, 2009) have been successfully employed to reduce the effect of physiological noise. Ironically, the presence of physiological noise alleviates the effect of sub-optimal imaging at low resolution, because the thermal noise plays a subordinate role altogether (Gonzalez-Castillo et al., 2011). This is, however, not the case at higher resolution. At high resolution, any reduction of SNR, e.g. due to suboptimal flip angles, reduces tSNR approximately proportionally.

Among others, SNR is proportional to the voxel volume (Edelstein et al., 1986). Thus, the increase of spatial resolution from $1 \mathrm{~mm}$ isotropic $\left(1 \mathrm{~mm}^{3}\right)$ to $0.8 \mathrm{~mm}$ isotropic $\left(0.512 \mathrm{~mm}^{3}\right)$ costs almost a factor of 2 in terms of SNR. But high resolution causes additional sensitivity reduction, because the image acquisition takes longer. This may reduce the temporal sampling rate and thus the number of sampling points per unit time, but a minimum number of independent observations is required to discriminate a real activation from spontaneous noisy fluctuations (Murphy et al., 2007). Additionally, it can cause sub-optimal choices of imaging parameters such as TE in the case of echo-train imaging sequences such as EPI. Moreover, partial Fourier or higher acceleration factors may be required to counteract the delay. This, however, comes at an SNR cost of the undersampling factor and an additional "geometry factor" (g-factor), which accounts for the introduction of noise from the imperfect disentanglement of spatial locations in the aliased, under-sampled images. Improvements in coil element decoupling and image reconstruction (Roemer et al., 1990; Triantafyllou et al., 2011; Sotiropoulos et al., 2013) as well as improved volumetric distribution of the aliases (Breuer et al., 2006; Bilgic et al., 2015) can reduce the g-factor penalty. These improvements can be particularly relevant to high resolution acquisitions because the distance between two aliased voxels is smaller if the voxel size is smaller (given a fixed matrix size). Two mechanisms can help to recover sensitivity in high resolution fMRI: 1) In smaller voxels, partial volume effects are reduced and distinct signals might emerge, which would otherwise be clouded by adjacent noise sources or cancel out with opposite signals (De Martino et al., 2011). 2) Small voxels reduce intra-voxel dephasing caused by strong macroscopic 
static field gradients (Young et al., 1988; Reichenbach et al., 1997). Using high resolution may therefore recover signal from areas with strong susceptibility differences, e.g. orbitofrontal cortex or near the ear canals (Weiskopf et al., 2006; Weiskopf et al., 2007)(but see also other approaches: z-shimming, e.g. Gu et al. (2002), and tailored RF pulses, e.g. Cho and Ro (1992)).

Long EPI echo-trains bring about another difficulty, namely image distortions. k-space lines are acquired at different echo times in an EPI train leading to differences in the accumulated phase of the signal depending on local offresonance frequency (Reichenbach et al., 1997). The image distortions hinder the precise co-registration with anatomical reference images which are typically acquired with a $T_{1}$ weighted 3D magnetizationprepared rapid gradient-echo imaging sequence (MPRAGE, Mugler and Brookeman, 1990) or more recent sequence variant such as MP2RAGE (Marques et al., 2010) or MEMPR (van der Kouwe et al., 2008). The latter variants are more apt to counter RF inhomogeneity (but see also Van de Moortele et al., 2009), which is problematic at ultra-high field ${ }^{5}$. The mismatch between structural images and fMRI data can severely limit the interpretability of high-resolution $\mathrm{fMRI}$ results because the spatial extent of the investigated structures may only be a few voxels. For example, if the cortical thickness is assumed to be $3 \mathrm{~mm}$, a misplacement of one $0.75 \mathrm{~mm}$ voxel will misattribute the cortical depth by $25 \%$, which is the thickness of at least one cortical layer. Susceptibility induced image distortions can be alleviated in the image-reconstruction or by post-processing techniques. An additional $B_{0}$ field map (Hutton et al., 2002; Cusack et al., 2003), a reversed or alternated phase-encoding scheme (Andersson et al., 2003; Smith et al., 2004), or reference scans (Zaitsev et al., 2004; Chung et al., 2011; Dragonu et al., 2013) are required. Recent developments in this field are further discussed in the discussion chapter.

Finally, it must be reiterated that ultra-high field scanners afford higher image SNR (Uğurbil et al., 2003 and references therein; Pohmann et al., 2015), because more spins contribute to the available magnetization. This

\footnotetext{
${ }^{5}$ The term ultra-high field refers to field strengths at which the Larmor frequency of hydrogen falls into the range of ultra-high frequency (UHF), $300 \mathrm{Mhz}-$ $3000 \mathrm{MHz}$, which is the case at $7 \mathrm{~T}$ and above.
} 
is the case because less spins occupy the state anti-parallel to the external magnetic field. BOLD imaging receives additional gains because the effect size increases with field strength, so that overall, a supra-linear increase of CNR with field strength is observable for both $T_{2}$ and $T_{2}{ }^{*}$ weighted BOLD, irrespective of their changes in sensitivity with field strength, which are described above. Since physiological noise ultimately limits the achievable tSNR, the increased SNR from ultra-high field is well invested to counter the SNR reduction by high spatial resolution.

\section{Point-spread function in the presence of signal decay during echo- train sequences}

High spatial resolution acquisitions using EPI require long echo trains to sample all k-space lines. The acceleration techniques mentioned in the previous section can reduce the required number of lines. However, the detrimental effect on image SNR has also been mentioned. Besides distortions, long echo trains can also have a detrimental effect on the image point spread function. The latter will be formally introduced in the following section.

In the most common imaging technique in MRI, k-space signals $s(\vec{k})$ are acquired and Fourier transformed to obtain an image,

$$
\begin{gathered}
s(\vec{k})=\int \rho(\vec{r}) \exp (i \vec{k} \vec{r}) d \vec{r} \\
I(\vec{r})=\mathcal{F}(s(\vec{k}))=\frac{1}{2 \pi} \int\left(\int \rho\left(\vec{r}^{\prime}\right) \exp \left(i \vec{k} \vec{r}^{\prime}\right) d \vec{r}^{\prime}\right) \exp (-i \vec{k} \vec{r}) d \vec{k} \\
=\rho(\vec{r}),
\end{gathered}
$$


where $\rho(\vec{r})$ is the measurable spin-density ${ }^{6}$ at a given point $\vec{r}$, and the integral is evaluated in all space (be it $2 \mathrm{D}$ or $3 \mathrm{D}$ encoding). If the k-space signal is modulated by a filter (modulation transfer function, MTF),

$$
s^{\prime}(\vec{k})=m(\vec{k}) s(\vec{k})
$$

the resultant image is as the convolution of the Fourier transform of $\mathrm{k}$ space data and the Fourier transform of the filter function ${ }^{7}$ (convolution theorem),

$$
\begin{aligned}
I(\vec{r})=\mathcal{F}\left(s^{\prime}(\vec{k})\right)= & \mathcal{F}(s(\vec{k}) m(\vec{k}))=\mathcal{F}(s(\vec{k})) * \mathcal{F}(m(\vec{k})) \\
& =\rho(\vec{r}) * \mathcal{F}(m(\vec{k})) .
\end{aligned}
$$

This is the defining equation for the point spread function (PSF): The PSF is the function, which is convolved with the ideal measurable spin-density yielding the resultant image (Rossmann, 1969; Robson et al., 1997),

$$
I(\vec{r})=\int \rho\left(\vec{r}^{\prime}\right) \operatorname{PSF}\left(\vec{r}-\vec{r}^{\prime}\right) d \vec{r}^{\prime} .
$$

For example, the image resolution is limited by the maximum extent of sampled k-space, which can be formalized by the application of a boxcar filter $m_{\text {trunc }}\left(k_{x}, k_{y}\right)$ truncating the sampling of the infinite $\mathrm{k}$-space in a $2 \mathrm{D}$ Cartesian grid:

$$
m_{\text {trunc }}\left(k_{x}, k_{y}\right)= \begin{cases}1 & \text { if }\left|k_{x}\right|<k_{x, \max },\left|k_{y}\right|<k_{y, \max }, \\ 0 & \text { otherwise }\end{cases}
$$

The Fourier transform of $m_{\text {trunc }}\left(k_{x}, k_{y}\right)$ is a sinc function, and hence, any edge or peak in the image has attenuated side lobes. This phenomenon is known as Gibbs ringing and is exemplified qualitatively in Figure 1-1 a.

\footnotetext{
${ }^{6}$ The measurable spin-density takes contrast preparation (e.g. $\mathrm{T}_{2}{ }^{*}$ weighting) into consideration, but constant, global scaling factors are neglected here for simplicity. In the literature, such scaling factors may also be attributed to the point spread function.

${ }^{7}$ Leaving more complex dependencies aside. I.e., it is assumed that the image is composed of isoplanatic patches, that is areas that are approximately shiftinvariant, see (Rossmann, 1969) and (Robson et al., 1997).
} 
When k-space lines are acquired consecutively in echo train sequences, attenuation due to signal decay occurs. In the case of an EPI sequence with linear phase-encoding, the modulation is

$$
\begin{aligned}
m_{T_{2}^{*}}\left(k_{x}, k_{y}\right)= & \exp \left(-\frac{t_{e s} n \Delta k_{y}+t_{s} m \Delta k_{x}+\text { const. }}{T_{2}^{*}}\right) \\
& =m_{T_{2}^{*}}\left(k_{x}(m), k_{y}(n)\right)
\end{aligned}
$$

where $t_{e s}$ is the echo spacing and $t_{s}$ is the sampling time within one readout. $\Delta k_{y}$ and $\Delta k_{x}$ denote the sampling step sizes and $n$ and $m$ denote sampling indices in the respective direction. The constant term stems from a non-zero starting time and may be ignored further, because it does not modulate the sampling differentially. Since the sampling time is typically much smaller than $\mathrm{T}_{2}{ }^{*}$ and the echo spacing $\left(t_{s} \ll t_{e s}, t_{s} \ll T_{2}^{*}\right)$ its effect can be ignored in an approximation. Then, the modulation occurs only in phase-encoding direction $\mathrm{y}$. In this approximation, the effect of $\mathrm{T}_{2}{ }^{*}$ decay on the phase-encoding direction can be treated analogously to the effect of $\mathrm{T}_{2}{ }^{*}$ decay on the read-direction in a simple gradient-echo imaging sequence (with a long read-out) and the combined effect of truncation and $\mathrm{T}_{2}{ }^{*}$ decay is (Haacke et al., 1999 eq. 13.54; Qin, 2012 Eq. 17)

$$
P S F_{\text {trunc }+T_{2}^{*}}(y) \propto \frac{\exp \left(-i \pi \frac{y}{\Delta y}\right)-\exp \left(i \pi \frac{y}{\Delta y}\right) \exp \left(-\frac{T_{s}}{T_{2}^{*}}\right)}{\frac{1}{2 \pi} \frac{T_{s}}{T_{2}^{*}}-i \frac{y}{\Delta y}}
$$

In the above equation any constant scaling factors were omitted for the sake of clarity. As can be seen, the asymmetry of the $T_{2}{ }^{*}$ decay during the acquisition time $T_{S}$ with linear ordering yields a complex PSF (Figure 1-1 b). The expression yields the pure sinc-modulation of the truncation when $\lim \left(T_{s} / T_{2}^{*}\right) \rightarrow 0$.

Equation $\left({ }^{* *}\right)$ is also applicable to the case of $\mathrm{T}_{2}$ decay along a multi-echo spin echo sequence like RARE or HASTE with linear ordering when replacing $T_{2}$ for $T_{2}{ }^{*} . T_{s}$ then stands for the time between consecutive spin-echoes (often labeled $2 \tau$ ). Finally, with these replacements, this filter is also appropriate for the partition direction of the 3D-GRASE sequence employed in this thesis when using linear ordering. When using center-out ordering (that is $k_{0}, k_{-1}, k_{1}, k_{-2}, k_{2}, \ldots$ ), the modulation is nearly symmetric 
about the k-space center and the resulting PSF can be approximated by Qin, 2012 (Qin, 2012 Eq. 22)

$$
P S F_{\text {trunc }+T_{2}^{*}}(y) \propto \frac{\frac{1}{\pi} \frac{T_{s}}{T_{2}^{*}}+\exp \left(-\frac{T_{s}}{T_{2}^{*}}\right)\left[\frac{y}{\Delta y} \sin \left(\pi \frac{y}{\Delta y}\right)-\frac{1}{\pi} \frac{T_{s}}{T_{2}^{*}} \cos \left(\pi \frac{y}{\Delta y}\right)\right]}{\left(\frac{1}{\pi} \frac{T_{s}}{T_{2}^{*}}\right)^{2}+\left(\frac{y}{\Delta y}\right)^{2}}
$$

Last, the case of a spin-echo or a spin-echo EPI shall be considered. It should be noted that typical depictions of the signal evolution around the spin-echo can be misleading because they might suggest a symmetric envelop due to $\mathrm{T}_{2}{ }^{*}$ decay around the echo. Rather, the signal amplitude is as follows (Haacke et al., 1999 Eq. 8.18):

$$
s(t)=s(0) \begin{cases}\exp \left(-\frac{t}{T_{2}}\right) \exp \left(-\frac{T E-t}{T_{2}^{\prime}}\right) & \text { for } \tau<t<2 \tau=T E \\ \exp \left(-\frac{t}{T_{2}}\right) \exp \left(-\frac{t-T E}{T_{2}^{\prime}}\right) & \text { for } t>2 \tau=T E \\ =\exp \left(-\frac{t}{T_{2}^{*}}\right) \exp \left(-\frac{T E}{T_{2}^{\prime}}\right) & \end{cases}
$$

where $\tau$ is the delay between excitation and refocusing pulse, and

$$
\frac{1}{T_{2}^{*}}=\frac{1}{T_{2}}+\frac{1}{T_{2}^{\prime}}
$$

The approximation of a symmetric signal evolution is only justified when $T_{2}^{*} \ll T_{2}$, and the signal drops off quickly on the sides. For brain parenchyma, this is not the case, because $T_{2}^{*} \approx 0.5 T_{2}$. In that case, the branch before the spin-echo is almost constant in signal amplitude. In the example in Figure 1-1 c, $T_{2}^{*} / T_{2}=1 / 3$ (blue) and $T_{2}^{*} / T_{2}=1 / 6$ were chosen.

Again, in consequence of the asymmetric decay, the resulting PSF is complex. The complex nature of the PSF is somewhat difficult to grasp, which is why often in the literature and also in this thesis (but see Huber et al., 2015b), a simplified and more practical approach using the magnitude of the PSF is used. A complex PSF entails that the effect on adjacent voxels in the (magnitude) image must also be considered as a vector sum in the complex plane. 
Although analytical expressions for the above PSFs may be derived using Fourier transforms and the convolution theorem, a numerical treatment of these effects is convenient, because it flexibly allows studying e.g. different ordering schemes or the effect of random noise contributions. Further, it is well suitable to account for the discrete sampling. Also, the analytical expressions can quickly become complicated diminishing their elegance and clarity. The truncation effect can be mimicked easily by performing the calculations on a much higher spatial resolution than the actual imaging resolution, e.g. $1 \mu \mathrm{m}$. Then, only the central part of k-space is filled. This is important when considering multiple filters at once. For example, partial Fourier acquisition using zero-filling misses up to half of the k-space required for the imaging resolution (Feinberg et al., 1986; Margosian et al., 1986). This fraction, however, becomes significantly smaller when working in the high resolution ( $1 \mu \mathrm{m}-)$ space. In advanced image reconstruction, the (idealized) conjugate symmetry of k-space of a real object is exploited and the missing k-space information is filled by known data from the opposite site (Haacke et al., 1991; Noll et al., 1991; McGibney et al., 1993). In theory, these techniques could recover the full nominal resolution, but because of the experimental imperfections in the acquired data, the effect on the PSF is not easily quantifiable.

Finally, it may be noted that the same framework is used to assess and correct for image distortions in PSF mapping techniques for EPI (Robson et al., 1997; Zeng and Constable, 2002; Zaitsev et al., 2004). In this approach, the spatially dependent off-resonance contributes a complex phase in the integral in equation $(*)$ in addition to the intended $\vec{k}$ encoding:

$$
s(\vec{k})=\int \rho(\vec{r}) \exp \left(i\left(\vec{k}+\overrightarrow{k^{\prime}}\right) \vec{r}\right) d \vec{r}
$$

In consequence, the signal is misplaced in the resulting image (Reichenbach et al., 1997). The off-resonance has a stronger effect on the phase-encoding direction than on read-direction because of the different time-scales at which they are sampled. The fact that the PSF has a finite spread is disregarded in these distortion-correction methods because the shift is modelled only by the shift of the peak intensity in the PSF mapping data. 

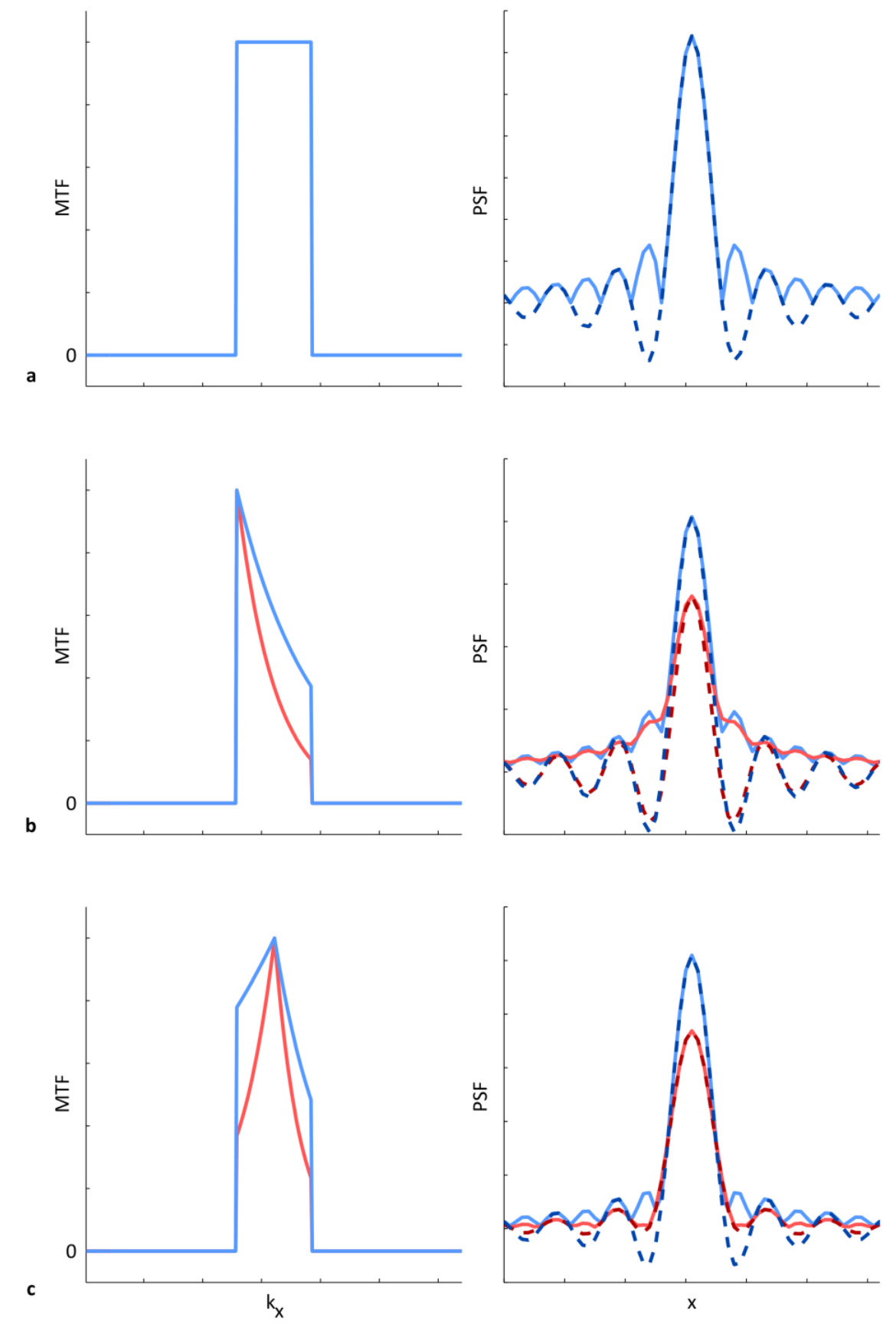
Figure 1-1: Qualitative examples of MTFs (left) and corresponding PSFs (right) for different filters. The PSF plots are zoomed in $x$ to the center. a) truncation without any decay, b) truncation and asymmetric exponential decay (e.g. phase-encoding direction in EPI, partition direction in 3D-GRASE with linear ordering), c) phase-encoding of spin-echo EPI experiment. Solid lines indicate magnitudes, dashed, darker lines indicate the real part of the PSFs.

\section{D-GRASE}

The superior functional specificity of $T_{2}$ weighted BOLD compared to $\mathrm{T}_{2}{ }^{*}$ weighted BOLD as well as the detrimental effect of long echo trains on image distortions and blurring have been described above. Besides accelerated imaging and partial Fourier acquisitions, reduced field of view (FoV) acquisitions can be employed to reduce the duration of the echo train. Various techniques to achieve reduced fields of view exist and are discussed in Chapter 2, but because of its high relevance to this thesis, the $3 D$ inner volume gradient and spin-echo (3D-GRASE) sequence (Feinberg et al., 2008) will be described in more detail in this section. GRASE combines two different fast imaging techniques, EPI and rapid acquisition with relaxation enhancement (RARE) (Hennig et al., 1986), nowadays better known as turbo-spin echo (TSE) or fast spin echo (FSE). In RARE, multiple pairs of refocusing $\mathrm{RF}$ pulses and readouts are applied after a given excitation instead of a single pair. This approach reduces the acquisition time of $\mathrm{T}_{2}$ weighted images immensely. In GRASE, multiple echoes are acquired after each refocusing pulse in an EPI fashion (Feinberg and Oshio, 1991) accelerating the acquisition even further because less refocusing pulses are needed than in RARE. Volumetric acquisition can be achieved in a 2D multi-slice or 3D approach. In anatomical imaging, the EPI factors are typically held modest, and a small, odd number of gradient-echoes (e.g. 3 or 5) are acquired between consecutive refocusing pulses (Trampel et al., 2013). Each gradient-echo receives its own phase-encoding via pre-phaser gradients and phase-encoding gradient blips. While the signal phase is refocused at the central spin-echoes throughout the echo train, this is not the case for the non-central gradient-echoes in the case of off-resonance (Figure 1-2 b). Also, the $\mathrm{T}_{2}$ and $\mathrm{T}_{2}{ }^{*}$ weighting varies between the echoes (see Figure 1-2 a, c). Many phase-encoding strategies have been suggested to address these issues, see (Bernstein et al., 2004) for details. 
a

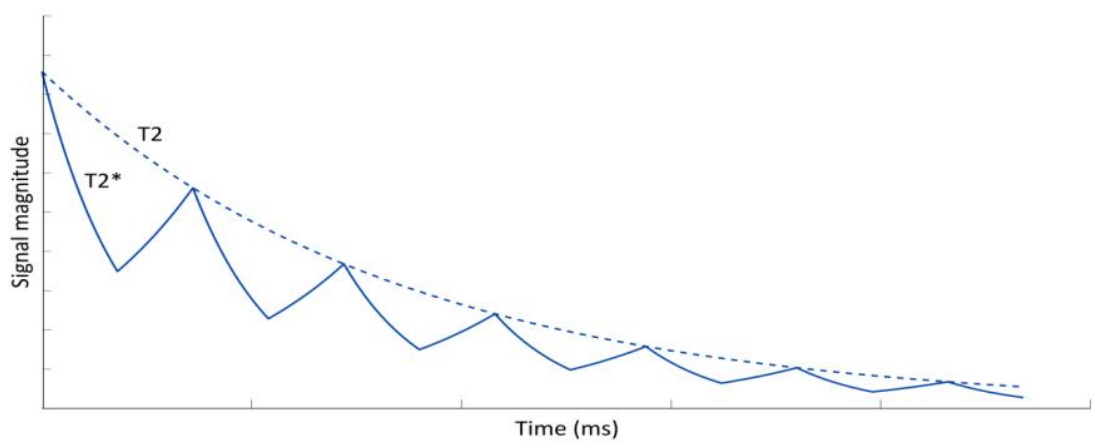

b
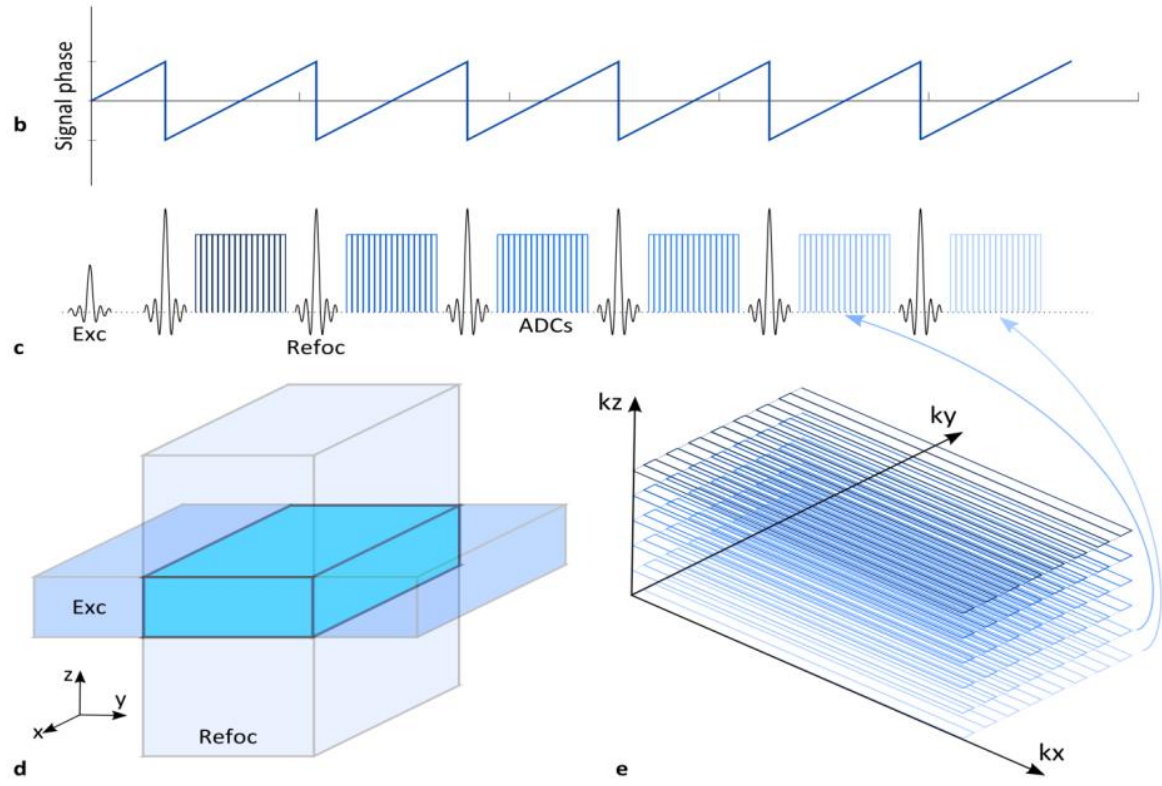

Figure 1-2: 3D-GRASE sequence diagrams (not to scale). a) Signal attenuation due to $T_{2}$ (dashed line) and combined effect of $T_{2}$ and $T_{2}{ }^{*}$ (solid line) throughout the readout. b) Phase evolution in the presence due to off-resonance. c) Simplified pulse diagram only displaying the excitation (Exc) and refocusing pulses (Refoc) and readouts (ADCs) without gradient activity shown. Diagram is not to scale. d) Inner volume selection mechanism: The selected volume is the mutual volume of excited and refocused slabs. e) Exemplary 3D k-space readout. Linear ordering in kzdirection is shown. Color-coding corresponds to the temporal order in $\mathbf{c}$.

Two unique features separate the 3D-GRASE technique employed in this thesis from most anatomical acquisition techniques and those for perfusion imaging, in which GRASE is also often utilized. First, the 3D-GRASE is a single-shot technique in which the entire volume is read out after a single 
excitation pulse. Second, in order to keep the duration of the acquisition short, it uses inner volume selective refocusing pulses to reduce the field of view in the phase encoding direction. The latter is done as follows: For excitation, a slab-selective $90^{\circ} \mathrm{RF}$ pulse is used. The slab-selective refocusing pulses are then executed orthogonally to the excitation pulse plane. In consequence, only the mutual volume receives both excitation and refocusing pulses and produces echoes when the read-out is performed (Figure 1-2 d). Two of the three spatial dimensions are thus limited because any tissue outside the mutual volume is either not excited or not refocused. These two imaging directions are well suited for phaseencoding (primary and secondary phase-encoding direction, or in-plane direction and partition direction phase-encoding), while the third direction is sampled as the read-direction (frequency encoding direction, $x$-direction in Figure 1-2 e). The in-plane phase encoding is linear as in an EPI whereas the partition direction is encoded in center-out or linear fashion. In either case, the signal magnitude and phase do not experience any discontinuities due to off-resonance or different decay due to $T_{2}$ and $T_{2}{ }^{*}$ but a smooth evolution along the two directions. The effect of these modulations on the imaging point spread function is described in the section above. Figure 1-3 shows a more detailed sequence pulse diagram including gradients. 
Chapter 1

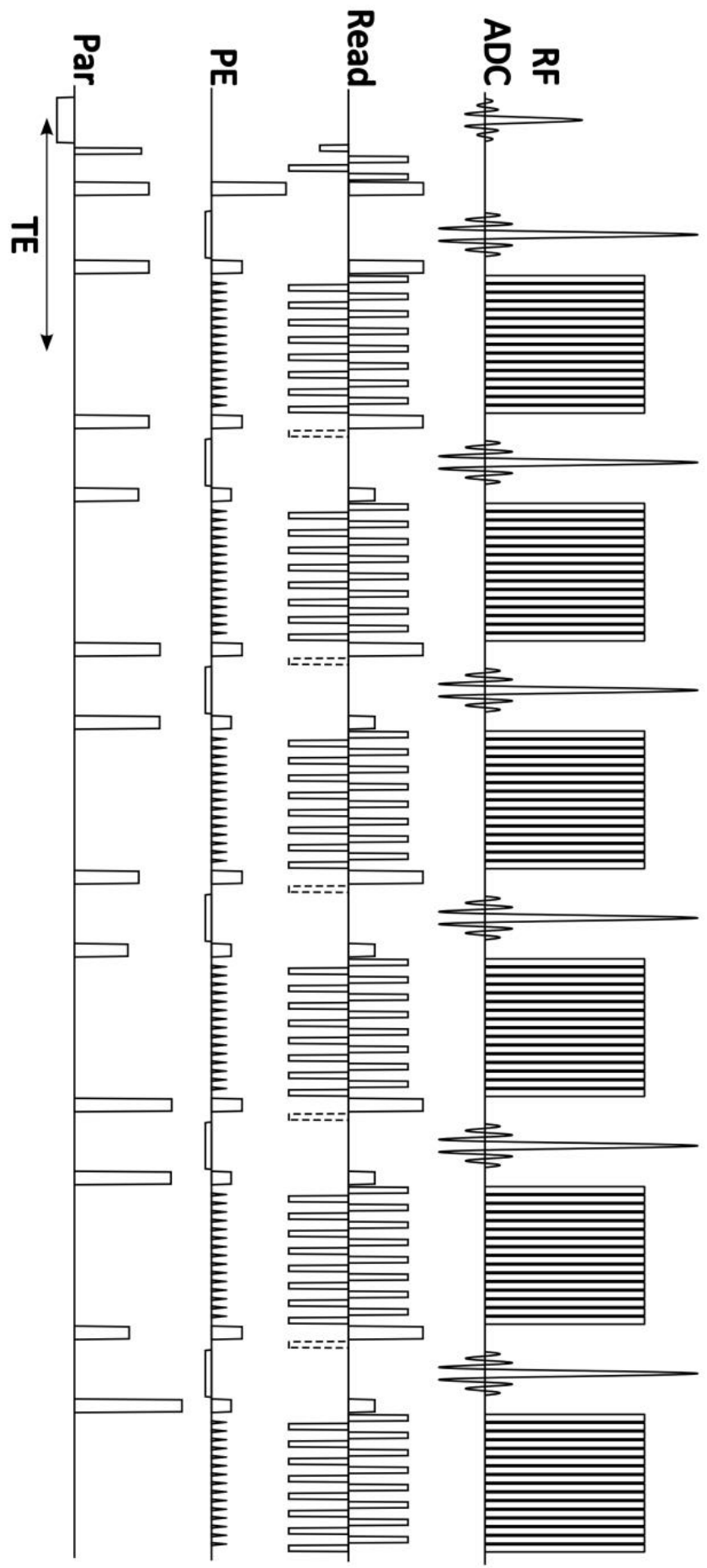


Figure 1-3: 3D-GRASE sequence pulse diagram (not to scale). Note that slab-selective gradients for excitation and refocusing are executed in partition (Par) and phase-encoding (PE) direction, respectively. For $\mathrm{T}_{2}$ weighting, a short delay time is required after each EPI readout, indicated by the dashed additional read-out gradients. Crusher and pre/post-phaser gradients were combined for increased speed. Three phasecorrection scans are acquired immediately after the excitation. Center-out partition encoding is displayed.

\section{Chapter overview}

The introduction outlined the scientific background for the following chapters of this thesis including the basics of MRI, the BOLD effect, neurovascular coupling, the functional cerebral organization at the mesoscale, imaging considerations for high resolution fMRI, the image point spread function, and the 3D-GRASE imaging pulse sequence. All following results refer to high resolution human $\mathrm{MRI}$ at ultra-high field. In Chapter 2 we report the results of a comparison of two $T_{2}$ weighted imaging sequences for application in high resolution $\mathrm{FMRI}$, spin-echo EPI and 3D-GRASE, at $7 \mathrm{~T}$. The main findings are that 3D-GRASE offers better functional sensitivity and specificity than SE-EPI while its spatial coverage and imaging acuity are limited. For these reasons, 3D-GRASE was further developed employing variable flip-angle refocusing pulses to increase coverage and improve the imaging PSF. The results are reported in Chapter 3. In Chapter 4, these methodological advances are put into practice in a high-resolution 9.4 $\mathrm{T}$ experiment. We performed population receptive field mapping demonstrating the capability to perform routine visual experiments with high quality results at 9.4 T. High-resolution $\mathrm{T}_{2}{ }^{*}$ weighted anatomical imaging showed differences in cortical depth profiles between visual areas V1 and V2. These results are paired with preliminary results of ocular dominance using 3D-GRASE. Finally, a few aspects of high-resolution fMRI acquisition and analysis are discussed in Chapter 5, partly incorporating the results of the reported studies. 


\section{References}

Adams, D.L., and Horton, J.C. (2009). Ocular dominance columns: enigmas and challenges. Neuroscientist 15, 62-77. doi: $10.1177 / 1073858408327806$.

Adams, D.L., Sincich, L.C., and Horton, J.C. (2007). Complete pattern of ocular dominance columns in human primary visual cortex. Journal of Neuroscience 27, 10391-10403. doi: 10.1523/jneurosci.292307.2007.

Andersson, J.L., Skare, S., and Ashburner, J. (2003). How to correct susceptibility distortions in spin-echo echo-planar images: application to diffusion tensor imaging. Neuroimage 20, 870-888. doi: 10.1016/s1053-8119(03)00336-7.

Attwell, D., Buchan, A.M., Charpak, S., Lauritzen, M., Macvicar, B.A., and Newman, E.A. (2010). Glial and neuronal control of brain blood flow. Nature 468, 232-243.

Bandettini, P.A., Wong, E.C., Hinks, R.S., Tikofsky, R.S., and Hyde, J.S. (1992). Time course EPI of human brain function during task activation. Magn Reson Med 25, 390-397. doi: 10.1002/mrm.1910250220.

Bandettini, P.A., Wong, E.C., Jesmanowicz, A., Hinks, R.S., and Hyde, J.S. (1994). Spin-Echo and Gradient-Echo Epi of Human Brain Activation Using Bold Contrast - a Comparative-Study at $1.5 \mathrm{~T}$. Nmr in Biomedicine 7, 12-20. doi: 10.1002/nbm.1940070104.

Belliveau, J.W., Kennedy, D.N., Jr., Mckinstry, R.C., Buchbinder, B.R., Weisskoff, R.M., Cohen, M.S., Vevea, J.M., Brady, T.J., and Rosen, B.R. (1991). Functional mapping of the human visual cortex by magnetic resonance imaging. Science 254, 716-719.

Bernstein, M.A., King, K.F., and Zhou, X.J. (2004). Handbook of MRI pulse sequences. Elsevier.

Besle, J., Sanchez-Panchuelo, R.M., Bowtell, R., Francis, S., and Schluppeck, D. (2014). Event-related fMRI at 7T reveals overlapping cortical representations for adjacent fingertips in $\mathrm{S} 1$ of individual subjects. Hum Brain Mapp 35, 2027-2043. doi: 10.1002/hbm.22310.

Bianciardi, M., Fukunaga, M., Van Gelderen, P., De Zwart, J.A., and Duyn, J.H. (2011). Negative BOLD-fMRI signals in large cerebral veins. J Cereb Blood Flow Metab 31, 401-412. doi: 10.1038/jcbfm.2010.164.

Bilgic, B., Gagoski, B.A., Cauley, S.F., Fan, A.P., Polimeni, J.R., Grant, P.E., Wald, L.L., and Setsompop, K. (2015). Wave-CAIPI for highly accelerated 3D imaging. Magnetic Resonance in Medicine 73, 21522162. 
Birn, R.M., Smith, M.A., Jones, T.B., and Bandettini, P.A. (2008). The respiration response function: the temporal dynamics of $\mathrm{fMRI}$ signal fluctuations related to changes in respiration. Neuroimage 40, 644-654. doi: 10.1016/j.neuroimage.2007.11.059.

Blamire, A.M., Ogawa, S., Ugurbil, K., Rothman, D., Mccarthy, G., Ellermann, J.M., Hyder, F., Rattner, Z., and Shulman, R.G. (1992). Dynamic mapping of the human visual cortex by high-speed magnetic resonance imaging. Proceedings of the National Academy of Sciences 89, 11069-11073.

Blasdel, G.G. (1992). Differential imaging of ocular dominance and orientation selectivity in monkey striate cortex. J Neurosci 12, 3115-3138.

Bonhoeffer, T., and Grinvald, A. (1991). Iso-orientation domains in cat visual cortex are arranged in pinwheel-like patterns. Nature 353, 429-431.

Boxerman, J.L., Bandettini, P.A., Kwong, K.K., Baker, J.R., Davis, T.L., Rosen, B.R., and Weisskoff, R.M. (1995a). The Intravascular Contribution to Fmri Signal Change - Monte-Carlo Modeling and DiffusionWeighted Studies in-Vivo. Magnetic Resonance in Medicine 34, 410. doi: DOI 10.1002/mrm.1910340103.

Boxerman, J.L., Hamberg, L.M., Rosen, B.R., and Weisskoff, R.M. (1995b). $\mathrm{Mr}$ Contrast Due to Intravascular Magnetic-Susceptibility Perturbations. Magnetic Resonance in Medicine 34, 555-566. doi: 10.1002/mrm.1910340412.

Boynton, G.M., Engel, S.A., Glover, G.H., and Heeger, D.J. (1996). Linear systems analysis of functional magnetic resonance imaging in human V1. The journal of neuroscience 16, 4207-4221.

Breuer, F.A., Blaimer, M., Mueller, M.F., Seiberlich, N., Heidemann, R.M., Griswold, M.A., and Jakob, P.M. (2006). Controlled aliasing in volumetric parallel imaging (2D CAIPIRINHA). Magn Reson Med 55, 549-556. doi: 10.1002/mrm.20787.

Broca, P. (1865). Sur le siège de la faculté du langage articulé. Bulletins de la Société d'Anthropologie de Paris 6, 377-393.

Brodmann, K. (1909). Vergleichende Lokalisationslehre der Groshirnrinde. Leipzig: Barth.

Buccino, G., Binkofski, F., Fink, G.R., Fadiga, L., Fogassi, L., Gallese, V., Seitz, R.J., Zilles, K., Rizzolatti, G., and Freund, H.J. (2001). Action observation activates premotor and parietal areas in a somatotopic manner: an fMRI study. European journal of neuroscience 13, 400404.

Buxton, R.B. (2001). The elusive initial dip. Neuroimage 13, 953-958. 
Buxton, R.B. (2012). Dynamic models of BOLD contrast. Neurolmage 62, 953-961. doi: 10.1016/j.neuroimage.2012.01.012.

Buxton, R.B., Wong, E.C., and Frank, L.R. (1998). Dynamics of blood flow and oxygenation changes during brain activation: the balloon model. Magnetic resonance in medicine 39, 855-864.

Chang, C., and Glover, G.H. (2009). Relationship between respiration, endtidal CO2, and BOLD signals in resting-state fMRI. Neuroimage 47, 1381-1393. doi: 10.1016/j.neuroimage.2009.04.048.

Cheng, K., Waggoner, R.A., and Tanaka, K. (2001). Human ocular dominance columns as revealed by high-field functional magnetic resonance imaging. Neuron 32, 359-374. doi: 10.1016/s08966273(01)00477-9.

Cho, Z., and Ro, Y. (1992). Reduction of susceptibility artifact in gradientecho imaging. Magnetic resonance in medicine 23, 193-200.

Chung, J.Y., In, M.H., Oh, S.H., Zaitsev, M., Speck, O., and Cho, Z.H. (2011). An improved PSF mapping method for EPI distortion correction in human brain at ultra high field (7T). MAGMA 24, 179-190. doi: 10.1007/s10334-011-0251-1.

Cusack, R., Brett, M., and Osswald, K. (2003). An evaluation of the use of magnetic field maps to undistort echo-planar images. Neuroimage $18,127-142$.

Da Costa, N.M., and Martin, K.A. (2010). Whose Cortical Column Would that Be? Front Neuroanat 4, 16. doi: 10.3389/fnana.2010.00016.

Damadian, R. (1971). Tumor detection by nuclear magnetic resonance. Science 171, 1151-1153.

Damadian, R., Minkoff, L., and Goldsmith, M. (1977). NMR in cancer: XXI. FONAR scan of the live human abdomen. Physiological chemistry and physics 10, 561-563.

Damasio, H., Grabowski, T., Frank, R., Galaburda, A.M., and Damasio, A.R. (1994). The return of Phineas Gage: clues about the brain from the skull of a famous patient. Science 264, 1102-1105.

De Martino, F., Esposito, F., Van De Moortele, P.F., Harel, N., Formisano, E., Goebel, R., Ugurbil, K., and Yacoub, E. (2011). Whole brain highresolution functional imaging at ultra high magnetic fields: an application to the analysis of resting state networks. Neuroimage 57, 1031-1044. doi: 10.1016/j.neuroimage.2011.05.008.

De Martino, F., Zimmermann, J., Muckli, L., Ugurbil, K., Yacoub, E., and Goebel, R. (2013). Cortical depth dependent functional responses in humans at 7T: improved specificity with 3D GRASE. PLoS One 8, e60514. doi: 10.1371/journal.pone.0060514. 
Dechent, P., and Frahm, J. (2000). Direct mapping of ocular dominance columns in human primary visual cortex. Neuroreport 11, 32473249.

Deyoe, E.A., Carman, G.J., Bandettini, P., Glickman, S., Wieser, J., Cox, R., Miller, D., and Neitz, J. (1996). Mapping striate and extrastriate visual areas in human cerebral cortex. Proceedings of the National Academy of Sciences 93, 2382-2386.

Donahue, M.J., Stevens, R.D., De Boorder, M., Pekar, J.J., Hendrikse, J., and Van Zijl, P.C. (2009). Hemodynamic changes after visual stimulation and breath holding provide evidence for an uncoupling of cerebral blood flow and volume from oxygen metabolism. Journal of Cerebral Blood Flow \& Metabolism 29, 176-185.

Douglas, R.J., and Martin, K. (1991). A functional microcircuit for cat visual cortex. The Journal of Physiology 440, 735-769.

Douglas, R.J., and Martin, K.A. (2004). Neuronal circuits of the neocortex. Annu. Rev. Neurosci. 27, 419-451.

Downing, P.E., Jiang, Y., Shuman, M., and Kanwisher, N. (2001). A cortical area selective for visual processing of the human body. Science 293, 2470-2473. doi: 10.1126/science.1063414.

Dragonu, I., Lange, T., Baxan, N., Snyder, J., Hennig, J., and Zaitsev, M. (2013). Accelerated point spread function mapping using signal modeling for accurate echo-planar imaging geometric distortion correction. Magn Reson Med 69, 1650-1656. doi: 10.1002/mrm.24396.

Duong, T.Q., Kim, D.S., Ugurbil, K., and Kim, S.G. (2001). Localized cerebral blood flow response at submillimeter columnar resolution. Proc Natl Acad Sci $U$ S A 98, 10904-10909. doi: 10.1073/pnas.191101098.

Duong, T.Q., Kim, D.S., Uğurbil, K., and Kim, S.G. (2000). Spatiotemporal dynamics of the BOLD fMRI signals: toward mapping submillimeter cortical columns using the early negative response. Magnetic resonance in medicine 44, 231-242.

Duong, T.Q., Yacoub, E., Adriany, G., Hu, X., Ugurbil, K., and Kim, S.G. (2003). Microvascular BOLD contribution at 4 and $7 \mathrm{~T}$ in the human brain: gradient-echo and spin-echo $\mathrm{fMRI}$ with suppression of blood effects. Magn Reson Med 49, 1019-1027. doi: 10.1002/mrm.10472.

Duvernoy, H.M., Delon, S., and Vannson, J. (1981). Cortical blood vessels of the human brain. Brain research bulletin 7, 519-579.

Edelstein, W.A., Glover, G.H., Hardy, C.J., and Redington, R.W. (1986). The intrinsic signal-to-noise ratio in NMR imaging. Magn Reson Med 3, 604-618. doi: 10.1002/mrm.1910030413. 
Engel, S.A., Glover, G.H., and Wandell, B.A. (1997). Retinotopic organization in human visual cortex and the spatial precision of functional MRI. Cerebral cortex 7, 181-192.

Engel, S.A., Rumelhart, D.E., Wandell, B.A., Lee, A.T., Glover, G.H., Chichilnisky, E.J., and Shadlen, M.N. (1994). fMRI of human visual cortex. Nature 369, 525. doi: 10.1038/369525a0.

Epstein, R., and Kanwisher, N. (1998). A cortical representation of the local visual environment. Nature 392, 598-601.

Ernst, T., and Hennig, J. (1994). Observation of a fast response in functional MR. Magnetic resonance in medicine 32, 146-149.

Feinberg, D.A., Hale, J.D., Watts, J.C., Kaufman, L., and Mark, A. (1986). Halving MR imaging time by conjugation: demonstration at $3.5 \mathrm{kG}$. Radiology 161, 527-531. doi: 10.1148/radiology.161.2.3763926.

Feinberg, D.A., Harel, N., Ramanna, S., Ugurbil, K., and Yacoub, E. (2008). Sub-millimeter Single-shot 3D GRASE with Inner Volume Selection for T2 weighted fMRI applications at 7 Tesla. 16th Annual Meeting International Society for Magnetic Resonance in Medicine, 2373.

Feinberg, D.A., Moeller, S., Smith, S.M., Auerbach, E., Ramanna, S., Gunther, M., Glasser, M.F., Miller, K.L., Ugurbil, K., and Yacoub, E. (2010). Multiplexed echo planar imaging for sub-second whole brain FMRI and fast diffusion imaging. PLoS One 5, e15710. doi: 10.1371/journal.pone.0015710.

Feinberg, D.A., and Oshio, K. (1991). GRASE (gradient- and spin-echo) MR imaging: a new fast clinical imaging technique. Radiology 181, 597602. doi: 10.1148/radiology.181.2.1924811.

Felleman, D.J., and Van Essen, D.C. (1991). Distributed hierarchical processing in the primate cerebral cortex. Cereb Cortex 1, 1-47.

Formisano, E., Kim, D.S., Di Salle, F., Van De Moortele, P.F., Ugurbil, K., and Goebel, R. (2003). Mirror-symmetric tonotopic maps in human primary auditory cortex. Neuron 40, 859-869.

Fox, P.T., and Raichle, M.E. (1986). Focal physiological uncoupling of cerebral blood flow and oxidative metabolism during somatosensory stimulation in human subjects. Proceedings of the National Academy of Sciences 83, 1140-1144.

Frahm, J., Bruhn, H., Merboldt, K.D., and Hänicke, W. (1992). Dynamic MR imaging of human brain oxygenation during rest and photic stimulation. Journal of Magnetic Resonance Imaging 2, 501-505.

Frahm, J., Haase, A., and Matthaei, D. (1986). Rapid three-dimensional MR imaging using the FLASH technique. Journal of computer assisted tomography 10, 363-368. 
Friston, K.J., Fletcher, P., Josephs, O., Holmes, A., Rugg, M.D., and Turner, R. (1998). Event-related fMRI: characterizing differential responses. Neuroimage 7, 30-40. doi: 10.1006/nimg.1997.0306.

Frost, M.A., and Goebel, R. (2012). Measuring structural-functional correspondence: spatial variability of specialised brain regions after macro-anatomical alignment. Neuroimage 59, 1369-1381. doi: 10.1016/j.neuroimage.2011.08.035.

Gati, J.S., Menon, R.S., Uğurbil, K., and Rutt, B.K. (1997). Experimental determination of the BOLD field strength dependence in vessels and tissue. Magnetic resonance in medicine 38, 296-302.

Geißler, A., Fischmeister, F.P.S., Grabner, G., Wurnig, M., Rath, J., Foki, T., Matt, E., Trattnig, S., Beisteiner, R., and Robinson, S.D. (2013). Comparing the microvascular specificity of the 3-and 7-T BOLD response using ICA and susceptibility-weighted imaging. Frontiers in human neuroscience 7.

Gilbert, C.D. (1983). Microcircuitry of the visual cortex. Annual review of neuroscience 6, 217-247.

Glover, G.H., Li, T.Q., and Ress, D. (2000). Image-based method for retrospective correction of physiological motion effects in $\mathrm{fMRI}$ : RETROICOR. Magnetic Resonance in Medicine 44, 162-167.

Goense, J., Merkle, H., and Logothetis, N.K. (2012). High-resolution fMRI reveals laminar differences in neurovascular coupling between positive and negative BOLD responses. Neuron 76, 629-639. doi: 10.1016/j.neuron.2012.09.019.

Goense, J.B.M., and Logothetis, N.K. (2006b). Laminar specificity in monkey V1 using high-resolution SE-fMRI. Magnetic Resonance Imaging 24, 381-392. doi: 10.1016/j.mri.2005.12.032.

Gonzalez-Castillo, J., Roopchansingh, V., Bandettini, P.A., and Bodurka, J. (2011). Physiological noise effects on the flip angle selection in BOLD fMRI. Neuroimage 54, 2764-2778. doi: 10.1016/j.neuroimage.2010.11.020.

Goodyear, B.G., and Menon, R.S. (2001). Brief visual stimulation allows mapping of ocular dominance in visual cortex using fMRI. Human Brain Mapping 14, 210-217. doi: 10.1002/hbm.1053.

Grinvald, A., Frostig, R.D., Siegel, R.M., and Bartfeld, E. (1991). Highresolution optical imaging of functional brain architecture in the awake monkey. Proceedings of the National Academy of Sciences 88, 11559-11563.

Grinvald, A., Lieke, E., Frostig, R.D., Gilbert, C.D., and Wiesel, T.N. (1986). Functional architecture of cortex revealed by optical imaging of intrinsic signals. 
Griswold, M.A., Jakob, P.M., Heidemann, R.M., Nittka, M., Jellus, V., Wang, J., Kiefer, B., and Haase, A. (2002). Generalized autocalibrating partially parallel acquisitions (GRAPPA). Magn Reson Med 47, 1202-1210. doi: 10.1002/mrm.10171.

Gu, H., Feng, H., Zhan, W., Xu, S., Silbersweig, D.A., Stern, E., and Yang, Y. (2002). Single-shot interleaved z-shim EPI with optimized compensation for signal losses due to susceptibility-induced field inhomogeneity at 3 T. Neuroimage 17, 1358-1364.

Haacke, E., Lindskogj, E., and Lin, W. (1991). A fast, iterative, partial-Fourier technique capable of local phase recovery. Journal of Magnetic Resonance (1969) 92, 126-145.

Haacke, E.M., Brown, R., Thompson, M., and Venkatesan, R. (1999). Magnetic resonance imaging: physical principles and sequence design. 1999. New York: A John Wiley and Sons.

Hahn, E. (1950a). Nuclear induction due to free Larmor precession. Physical Review 77, 297.

Hahn, E.L. (1950b). Spin echoes. Physical review 80, 580.

Hall, C.N., Reynell, C., Gesslein, B., Hamilton, N.B., Mishra, A., Sutherland, B.A., O'farrell, F.M., Buchan, A.M., Lauritzen, M., and Attwell, D. (2014). Capillary pericytes regulate cerebral blood flow in health and disease. Nature 508, 55-60. doi: 10.1038/nature13165.

Harel, N., Lin, J., Moeller, S., Ugurbil, K., and Yacoub, E. (2006). Combined imaging-histological study of cortical laminar specificity of fMRI signals. Neuroimage 29, 879-887. doi: 10.1016/j.neuroimage.2005.08.016.

Harlow, J.M. (1868). Recovery from the passage of an iron bar through the head. Publications of the Massachusetts Medical Society 2, $327-$ 246.

Hennig, J., Nauerth, A., and Friedburg, H. (1986). RARE imaging: a fast imaging method for clinical MR. Magn Reson Med 3, 823-833. doi: 10.1002/mrm.1910030602.

Horton, J., and Hedley-Whyte, E.T. (1984). Mapping of cytochrome oxidase patches and ocular dominance columns in human visual cortex. Philosophical Transactions of the Royal Society of London. Series B, Biological Sciences, 255-272.

Horton, J.C. (1984). Cytochrome oxidase patches: a new cytoarchitectonic feature of monkey visual cortex. Philosophical Transactions of the Royal Society of London. Series B, Biological Sciences, 199-253.

Horton, J.C., and Adams, D.L. (2005). The cortical column: a structure without a function. Philos Trans R Soc Lond B Biol Sci 360, 837-862. doi: $10.1098 /$ rstb.2005.1623. 
Hu, X., Le, T.H., Parrish, T., and Erhard, P. (1995). Retrospective estimation and correction of physiological fluctuation in functional MRI. Magnetic resonance in medicine 34, 201-212.

$\mathrm{Hu}, \mathrm{X}$. , and Yacoub, E. (2012). The story of the initial dip in fMRI. Neurolmage 62, 1103-1108. doi: 10.1016/j.neuroimage.2012.03.005.

Hua, J., Jones, C.K., Qin, Q., and Van Zijl, P.C. (2013a). Implementation of vascular-space-occupancy MRI at 7T. Magn Reson Med 69, 10031013. doi: 10.1002/mrm.24334.

Hubel, D., and Wiesel, T. (1963). Shape and arrangement of columns in cat's striate cortex. The Journal of physiology 165, 559-568.

Hubel, D.H., and Wiesel, T.N. (1962). Receptive fields, binocular interaction and functional architecture in the cat's visual cortex. The Journal of physiology 160, 106.

Hubel, D.H., and Wiesel, T.N. (1965). Receptive fields and functional architecture in two nonstriate visual areas (18 and 19) of the cat. Journal of neurophysiology 28, 229-289.

Hubel, D.H., and Wiesel, T.N. (1968). Receptive fields and functional architecture of monkey striate cortex. The Journal of physiology 195, 215-243.

Hubel, D.H., and Wiesel, T.N. (1974). Sequence regularity and geometry of orientation columns in the monkey striate cortex. Journal of Comparative Neurology 158, 267-293.

Huber, L., Goense, J., Kennerley, A.J., Trampel, R., Guidi, M., Reimer, E., Ivanov, D., Neef, N., Gauthier, C.J., Turner, R., and Moller, H.E. (2015a). Cortical lamina-dependent blood volume changes in human brain at 7 T. Neuroimage 107, 23-33. doi: 10.1016/j.neuroimage.2014.11.046.

Huber, L., Guidi, M., Goense, J., Mildner, T., Trampel, R., Schulz, J., Eichner, C., Turner, R., and Möller, H.E. (2015b). The magnitude point spread function is an inadequate measure of T2*-blurring in EPI. 23nd Annual Meeting of ISMRM 23, 2056.

Huber, L., Ivanov, D., Krieger, S.N., Streicher, M.N., Mildner, T., Poser, B.A., Moller, H.E., and Turner, R. (2013). Slab-selective, BOLD-corrected VASO at 7 tesla provides measures of cerebral blood volume reactivity with high signal-to-noise ratio. Magn Reson Med. doi: 10.1002/mrm.24916.

Hutton, C., Bork, A., Josephs, O., Deichmann, R., Ashburner, J., and Turner, R. (2002). Image distortion correction in fMRI: A quantitative evaluation. Neuroimage 16, 217-240. doi: 10.1006/nimg.2001.1054. 
Jin, T., and Kim, S.-G. (2008a). Cortical layer-dependent dynamic blood oxygenation, cerebral blood flow and cerebral blood volume responses during visual stimulation. Neuroimage 43, 1-9. doi: 10.1016/j.neuroimage.2008.06.029.

Jin, T., and Kim, S.G. (2008b). Improved cortical-layer specificity of vascular space occupancy fMRI with slab inversion relative to spin-echo BOLD at 9.4 T. Neuroimage 40, 59-67. doi: 10.1016/j.neuroimage.2007.11.045.

Kanwisher, N., Mcdermott, J., and Chun, M.M. (1997). The fusiform face area: a module in human extrastriate cortex specialized for face perception. The Journal of Neuroscience 17, 4302-4311.

Koopmans, P.J., Barth, M., and Norris, D.G. (2010). Layer-Specific BOLD Activation in Human VI. Human Brain Mapping 31, 1297-1304. doi: 10.1002/hbm.20936.

Koopmans, P.J., Barth, M., Orzada, S., and Norris, D.G. (2011). Multi-echo fMRI of the cortical laminae in humans at 7 T. Neuroimage 56, 1276-1285. doi: 10.1016/j.neuroimage.2011.02.042.

Kumar, A., Welti, D., and Ernst, R. (1975). Imaging of macroscopic objects by NMR Fourier zeugmatography. Naturwissenschaften 62, 34-34.

Kundu, P., Inati, S.J., Evans, J.W., Luh, W.M., and Bandettini, P.A. (2012). Differentiating BOLD and non-BOLD signals in fMRI time series using multi-echo EPI. Neuroimage 60, 1759-1770. doi: 10.1016/j.neuroimage.2011.12.028.

Kwong, K.K., Belliveau, J.W., Chesler, D.A., Goldberg, I.E., Weisskoff, R.M., Poncelet, B.P., Kennedy, D.N., Hoppel, B.E., Cohen, M.S., Turner, R., and Et Al. (1992). Dynamic magnetic resonance imaging of human brain activity during primary sensory stimulation. Proc Natl Acad Sci U S A 89, 5675-5679. doi: 10.1073/pnas.89.12.5675.

Larkman, D.J., Hajnal, J.V., Herlihy, A.H., Coutts, G.A., Young, I.R., and Ehnholm, G. (2001). Use of multicoil arrays for separation of signal from multiple slices simultaneously excited. Journal of Magnetic Resonance Imaging 13, 313-317.

Lauterbur, P.C. (1973). Image formation by induced local interactions: examples employing nuclear magnetic resonance. Nature 242, 190191.

Lauterbur, P.C. (1974). Magnetic resonance zeugmatography. Pure and Applied Chemistry 40, 149-157.

Levay, S., Connolly, M., Houde, J., and Van Essen, D. (1985). The complete pattern of ocular dominance stripes in the striate cortex and visual field of the macaque monkey. The Journal of neuroscience $5,486-$ 501. 
Lotze, M., Erb, M., Flor, H., Huelsmann, E., Godde, B., and Grodd, W. (2000). $\mathrm{fMRI}$ evaluation of somatotopic representation in human primary motor cortex. Neuroimage 11, 473-481.

Lu, H., Golay, X., Pekar, J.J., and Van Zijl, P. (2003). Functional magnetic resonance imaging based on changes in vascular space occupancy. Magnetic Resonance in Medicine 50, 263-274.

Mansfield, P. (1977). Multi-planar image formation using NMR spin echoes. Journal of Physics C: Solid State Physics 10, L55. doi: 10.1088/00223719/10/3/004.

Margosian, P., Schmitt, F., and Purdy, D. (1986). Faster MR imaging: imaging with half the data. Health Care Instrum 1, 195.

Marques, J.P., Kober, T., Krueger, G., Van Der Zwaag, W., Van De Moortele, P.F., and Gruetter, R. (2010). MP2RAGE, a self bias-field corrected sequence for improved segmentation and T1-mapping at high field. Neuroimage 49, 1271-1281. doi: 10.1016/j.neuroimage.2009.10.002.

Mcgibney, G., Smith, M., Nichols, S., and Crawley, A. (1993). Quantitative evaluation of several partial Fourier reconstruction algorithms used in MRI. Magnetic resonance in medicine 30, 51-59.

Menon, R.S., Ogawa, S., Strupp, J.P., and Ugurbil, K. (1997). Ocular dominance in human V1 demonstrated by functional magnetic resonance imaging. Journal of Neurophysiology 77, 2780-2787.

Moeller, S., Yacoub, E., Olman, C.A., Auerbach, E., Strupp, J., Harel, N., and Ugurbil, K. (2010). Multiband multislice GE-EPI at 7 tesla, with 16fold acceleration using partial parallel imaging with application to high spatial and temporal whole-brain fMRI. Magn Reson Med 63, 1144-1153. doi: 10.1002/mrm.22361.

Mountcastle, V.B. (1957). Modality and topographic properties of single neurons of cat's somatic sensory cortex. J Neurophysiol 20, 408434.

Muckli, L., De Martino, F., Vizioli, L., Petro, L.S., Smith, F.W., Ugurbil, K., Goebel, R., and Yacoub, E. (2015). Contextual Feedback to Superficial Layers of V1. Current Biology.

Mugler, J.P., and Brookeman, J.R. (1990). Three-dimensional magnetization-prepared rapid gradient-echo imaging (3D MP RAGE). Magnetic Resonance in Medicine 15, 152-157.

Murphy, K., Bodurka, J., and Bandettini, P.A. (2007). How long to scan? The relationship between $\mathrm{fMRI}$ temporal signal to noise ratio and necessary scan duration. Neurolmage 34, 565-574. doi: 10.1016/j.neuroimage.2006.09.032.

Nieuwenhuys, R. (2013). "The myeloarchitectonic studies on the human cerebral cortex of the Vogt-Vogt school, and their significance for 
the interpretation of functional neuroimaging data," in Microstructural Parcellation of the Human Cerebral Cortex. Springer), 55-125.

Noll, D.C., Nishimura, D.G., and Macovski, A. (1991). Homodyne detection in magnetic resonance imaging. IEEE Trans Med Imaging 10, 154163. doi: $10.1109 / 42.79473$.

Norris, D.G. (2012). Spin-echo fMRI: The poor relation? Neuroimage 62, 1109-1115. doi: 10.1016/j.neuroimage.2012.01.003.

Ogawa, S., Lee, T.M., Kay, A.R., and Tank, D.W. (1990). Brain magnetic resonance imaging with contrast dependent on blood oxygenation. Proc Natl Acad Sci U S A 87, 9868-9872.

Ogawa, S., Tank, D.W., Menon, R., Ellermann, J.M., Kim, S.G., Merkle, H., and Ugurbil, K. (1992). Intrinsic signal changes accompanying sensory stimulation: functional brain mapping with magnetic resonance imaging. Proc Natl Acad Sci U S A 89, 5951-5955.

Olman, C.A., Harel, N., Feinberg, D.A., He, S., Zhang, P., Ugurbil, K., and Yacoub, E. (2012). Layer-specific fMRI reflects different neuronal computations at different depths in human V1. PLoS One 7, e32536. doi: 10.1371/journal.pone.0032536.

Panchuelo, R.M.S., Schluppeck, D., Harmer, J., Bowtell, R., and Francis, S. (2015). Assessing the Spatial Precision of SE and GE-BOLD Contrast at 7 Tesla. Brain topography 28, 62-65.

Park, J., Ronen, I., Kim, D., and Ugurbil, K. (2005). Spatial specificity of high resolution gradient echo (GE) BOLD and spin echo (SE) BOLD fMRI in cat visual cortex at 9.4 T. 13th Annual Meeting of ISMRM 31.

Parkes, L.M., Schwarzbach, J.V., Bouts, A.A., Deckers, R.H., Pullens, P., Kerskens, C.M., and Norris, D.G. (2005). Quantifying the spatial resolution of the gradient echo and spin echo BOLD response at 3 Tesla. Magn Reson Med 54, 1465-1472. doi: 10.1002/mrm.20712.

Pohmann, R., Speck, O., and Scheffler, K. (2015). Signal-to-noise ratio and MR tissue parameters in human brain imaging at 3, 7, and 9.4 tesla using current receive coil arrays. Magn Reson Med. doi: 10.1002/mrm.25677.

Polimeni, J.R., Fischl, B., Greve, D.N., and Wald, L.L. (2010). Laminar analysis of 7T BOLD using an imposed spatial activation pattern in human V1. Neuroimage 52, 1334-1346. doi: 10.1016/j.neuroimage.2010.05.005.

Poser, B.A., Koopmans, P.J., Witzel, T., Wald, L.L., and Barth, M. (2010). Three dimensional echo-planar imaging at 7 Tesla. Neuroimage 51, 261-266. doi: 10.1016/j.neuroimage.2010.01.108. 
Pruessmann, K.P., Weiger, M., Scheidegger, M.B., and Boesiger, P. (1999). SENSE: sensitivity encoding for fast MRI. Magnetic resonance in medicine 42, 952-962.

Qin, Q. (2012). Point spread functions of the T2 decay in k-space trajectories with long echo train. Magn Reson Imaging 30, 11341142. doi: 10.1016/j.mri.2012.04.017.

Ramón Y Cajal, S. (1899). Comparative study of the sensory areas of the human cortex.

Reichenbach, J.R., Venkatesan, R., Yablonskiy, D.A., Thompson, M.R., Lai, S., and Haacke, E.M. (1997). Theory and application of static field inhomogeneity effects in gradient-echo imaging. Journal of Magnetic Resonance Imaging 7, 266-279. doi: 10.1002/jmri.1880070203.

Ress, D., Glover, G.H., Liu, J., and Wandell, B. (2007). Laminar profiles of functional activity in the human brain. Neurolmage 34, 74-84. doi: 10.1016/j.neuroimage.2006.08.020.

Robson, M.D., Gore, J.C., and Constable, R.T. (1997). Measurement of the point spread function in MRI using constant time imaging. Magn Reson Med 38, 733-740. doi: 10.1002/mrm.1910380509.

Roemer, P., Edelstein, W., Hayes, C., Souza, S., and Mueller, O. (1990). The NMR phased array. Magnetic resonance in medicine 16, 192-225.

Roland, E., and Larsen, B. (1976). Focal increase of cerebral blood flow during stereognostic testing in man. Arch Neurol 33, 551-558.

Roland, P.E., Skinhoj, E., Lassen, N.A., and Larsen, B. (1980). Different cortical areas in man in organization of voluntary movements in extrapersonal space. J Neurophysiol 43, 137-150.

Rossmann, K. (1969). Point Spread-Function, Line Spread-Function, and Modulation Transfer Function: Tools for the Study of Imaging Systems 1. Radiology 93, 257-272.

Roy, C.S., and Sherrington, C. (1890). On the regulation of the blood-supply of the brain. The Journal of physiology 11, 85.

Setsompop, K., Gagoski, B.A., Polimeni, J.R., Witzel, T., Wedeen, V.J., and Wald, L.L. (2012). Blipped-controlled aliasing in parallel imaging for simultaneous multislice echo planar imaging with reduced g-factor penalty. Magn Reson Med 67, 1210-1224. doi: 10.1002/mrm.23097.

Shapley, R., and Hawken, M.J. (2011). Color in the cortex: single-and double-opponent cells. Vision research 51, 701-717.

Shmuel, A., Yacoub, E., Chaimow, D., Logothetis, N.K., and Ugurbil, K. (2007). Spatio-temporal point-spread function of fMRI signal in human gray matter at 7 Tesla. Neuroimage 35, 539-552. doi: 10.1016/j.neuroimage.2006.12.030. 
Shoham, D., Hübener, M., Schulze, S., Grinvald, A., and Bonhoeffer, T. (1997). Spatio-temporal frequency domains and their relation to cytochrome oxidase staining in cat visual cortex. Nature 385, 529533.

Siero, J.C., Hendrikse, J., Hoogduin, H., Petridou, N., Luijten, P., and Donahue, M.J. (2015). Cortical depth dependence of the BOLD initial dip and poststimulus undershoot in human visual cortex at 7 Tesla. Magn Reson Med 73, 2283-2295. doi: 10.1002/mrm.25349.

Silver, M.A., and Kastner, S. (2009). Topographic maps in human frontal and parietal cortex. Trends Cogn Sci 13, 488-495. doi: 10.1016/j.tics.2009.08.005.

Sincich, L.C., and Horton, J.C. (2005). The circuitry of V1 and V2: integration of color, form, and motion. Annu. Rev. Neurosci. 28, 303-326.

Smith, S.M., Jenkinson, M., Woolrich, M.W., Beckmann, C.F., Behrens, T.E., Johansen-Berg, H., Bannister, P.R., De Luca, M., Drobnjak, I., Flitney, D.E., Niazy, R.K., Saunders, J., Vickers, J., Zhang, Y., De Stefano, N., Brady, J.M., and Matthews, P.M. (2004). Advances in functional and structural MR image analysis and implementation as FSL. Neuroimage 23 Suppl 1, S208-219. doi: 10.1016/j.neuroimage.2004.07.051.

Sodickson, D.K., and Manning, W.J. (1997). Simultaneous acquisition of spatial harmonics (SMASH): fast imaging with radiofrequency coil arrays. Magnetic Resonance in Medicine 38, 591-603.

Sotiropoulos, S.N., Moeller, S., Jbabdi, S., Xu, J., Andersson, J.L., Auerbach, E.J., Yacoub, E., Feinberg, D., Setsompop, K., Wald, L.L., Behrens, T.E., Ugurbil, K., and Lenglet, C. (2013). Effects of image reconstruction on fiber orientation mapping from multichannel diffusion MRI: reducing the noise floor using SENSE. Magn Reson Med 70, 1682-1689. doi: 10.1002/mrm.24623.

Sun, P., Ueno, K., Waggoner, R.A., Gardner, J.L., Tanaka, K., and Cheng, K. (2007). A temporal frequency-dependent functional architecture in human V1 revealed by high-resolution fMRI. Nat Neurosci 10, 1404-1406. doi: 10.1038/nn1983.

Trampel, R., Reimer, E., Huber, L., Ivanov, D., Heidemann, R.M., Schafer, A., and Turner, R. (2013). Anatomical brain imaging at 7T using twodimensional GRASE. Magn Reson Med. doi: 10.1002/mrm.25047.

Triantafyllou, C., Hoge, R.D., Krueger, G., Wiggins, C.J., Potthast, A., Wiggins, G.C., and Wald, L.L. (2005). Comparison of physiological noise at $1.5 \mathrm{~T}, 3 \mathrm{~T}$ and $7 \mathrm{~T}$ and optimization of fMRI acquisition parameters. Neuroimage 26, 243-250. doi: 10.1016/j.neuroimage.2005.01.007. 
Triantafyllou, C., Polimeni, J.R., and Wald, L.L. (2011). Physiological noise and signal-to-noise ratio in $\mathrm{fMRI}$ with multi-channel array coils. Neuroimage 55, 597-606. doi: 10.1016/j.neuroimage.2010.11.084.

Ts'o, D.Y., Zarella, M., and Burkitt, G. (2009). Whither the hypercolumn? J Physiol 587, 2791-2805. doi: 10.1113/jphysiol.2009.171082.

Turner, R., Jezzard, P., Wen, H., Kwong, K.K., Le Bihan, D., Zeffiro, T., and Balaban, R.S. (1993). Functional mapping of the human visual cortex at 4 and 1.5 tesla using deoxygenation contrast EPI. Magn Reson Med 29, 277-279.

Twieg, D.B. (1983). The k-trajectory formulation of the NMR imaging process with applications in analysis and synthesis of imaging methods. Medical Physics 10, 610-621.

Uğurbil, K., Adriany, G., Andersen, P., Chen, W., Garwood, M., Gruetter, R., Henry, P.-G., Kim, S.-G., Lieu, H., and Tkac, I. (2003). Ultrahigh field magnetic resonance imaging and spectroscopy. Magnetic resonance imaging 21, 1263-1281.

Uludag, K. (2010). To dip or not to dip: reconciling optical imaging and fMRI data. Proc Natl Acad Sci U S A 107, E23; author reply E24. doi: 10.1073/pnas.0914194107.

Uludag, K., Dubowitz, D.J., and Buxton, R.B. (2005). "Principles of functional imaging of the brain.," in Clinical Magnetic Resonance Imaging (3rd Ed), eds. R.R. Edelmann, J.R. Hesselink \& M.B. Zlatkin. (England).

Uludag, K., Muller-Bierl, B., and Ugurbil, K. (2009). An integrative model for neuronal activity-induced signal changes for gradient and spin echo functional imaging. Neuroimage 48, 150-165. doi: 10.1016/j.neuroimage.2009.05.051.

Van De Moortele, P.F., Auerbach, E.J., Olman, C., Yacoub, E., Ugurbil, K., and Moeller, S. (2009). T1 weighted brain images at 7 Tesla unbiased for Proton Density, T2* contrast and RF coil receive B1 sensitivity with simultaneous vessel visualization. Neuroimage 46, 432-446. doi: 10.1016/j.neuroimage.2009.02.009.

Van Der Kouwe, A.J., Benner, T., Salat, D.H., and Fischl, B. (2008). Brain morphometry with multiecho MPRAGE. Neuroimage 40, 559-569. doi: 10.1016/j.neuroimage.2007.12.025.

Vogt, C., and Vogt, O. (1919). Allgemeine ergebnisse unserer hirnforschung. JA Barth.

Wandell, B.A., Dumoulin, S.O., and Brewer, A.A. (2007). Visual field maps in human cortex. Neuron 56, 366-383.

Watanabe, M., Bartels, A., Macke, J.H., Murayama, Y., and Logothetis, N.K. (2013). Temporal jitter of the BOLD signal reveals a reliable initial dip and improved spatial resolution. Curr Biol 23, 2146-2150. doi: 10.1016/j.cub.2013.08.057. 
Weiskopf, N., Hutton, C., Josephs, O., and Deichmann, R. (2006). Optimal EPI parameters for reduction of susceptibility-induced BOLD sensitivity losses: a whole-brain analysis at $3 \mathrm{~T}$ and $1.5 \mathrm{~T}$. Neuroimage 33, 493-504. doi: 10.1016/j.neuroimage.2006.07.029.

Weiskopf, N., Hutton, C., Josephs, O., Turner, R., and Deichmann, R. (2007). Optimized EPI for fMRI studies of the orbitofrontal cortex: compensation of susceptibility-induced gradients in the readout direction. Magnetic Resonance Materials in Physics, Biology and Medicine 20, 39-49.

Weliky, M., Bosking, W.H., and Fitzpatrick, D. (1996). A systematic map of direction preference in primary visual cortex.

Wernicke, C. (1874). Der aphasische Symptomencomplex: eine psychologische Studie auf anatomischer Basis. Cohn.

Wiesel, T., Hubel, D., and Lam, D. (1974). Autoradiographic demonstration of ocular-dominance columns in the monkey striate cortex by means of transneuronal transport. Brain research 79, 273-279.

Wiggins, G.C., Polimeni, J.R., Potthast, A., Schmitt, M., Alagappan, V., and Wald, L.L. (2009). 96-Channel receive-only head coil for 3 Tesla: design optimization and evaluation. Magn Reson Med 62, 754-762. doi: 10.1002/mrm.22028.

Wilms, M., Eickhoff, S.B., Homke, L., Rottschy, C., Kujovic, M., Amunts, K., and Fink, G.R. (2010). Comparison of functional and cytoarchitectonic maps of human visual areas V1, V2, V3d, V3v, and V4(v). Neuroimage 49, 1171-1179. doi: 10.1016/j.neuroimage.2009.09.063.

Yacoub, E., Harel, N., and Ugurbil, K. (2008). High-field fMRI unveils orientation columns in humans. Proc Natl Acad Sci U S A 105, 10607-10612. doi: 10.1073/pnas.0804110105.

Yacoub, E., Shmuel, A., Logothetis, N., and Ugurbil, K. (2007). Robust detection of ocular dominance columns in humans using Hahn Spin Echo BOLD functional MRI at 7 Tesla. Neuroimage 37, 1161-1177. doi: 10.1016/j.neuroimage.2007.05.020.

Yacoub, E., Shmuel, A., Pfeuffer, J., Van De Moortele, P.F., Adriany, G., Ugurbil, K., and $\mathrm{Hu}, \mathrm{X}$. (2001). Investigation of the initial dip in $\mathrm{fMRI}$ at 7 Tesla. NMR Biomed 14, 408-412.

Yacoub, E., Ugurbil, K., and Harel, N. (2006). The spatial dependence of the poststimulus undershoot as revealed by high-resolution BOLD- and CBV-weighted fMRI. J Cereb Blood Flow Metab 26, 634-644. doi: 10.1038/sj.jcbfm.9600239.

Yacoub, E., Van De Moortele, P.F., Shmuel, A., and Ugurbil, K. (2005). Signal and noise characteristics of Hahn SE and GE BOLD fMRI at $7 \mathrm{~T}$ in 
humans. Neuroimage 24, 738-750. doi:

10.1016/j.neuroimage.2004.09.002.

Young, I., Cox, I., Bryant, D., and Bydder, G. (1988). The benefits of increasing spatial resolution as a means of reducing artifacts due to field inhomogeneities. Magnetic resonance imaging 6, 585-590.

Zaitsev, M., Hennig, J., and Speck, O. (2004). Point spread function mapping with parallel imaging techniques and high acceleration factors: fast, robust, and flexible method for echo-planar imaging distortion correction. Magn Reson Med 52, 1156-1166. doi: 10.1002/mrm.20261.

Zappe, A.C., Pfeuffer, J., Merkle, H., Logothetis, N.K., and Goense, J.B. (2008). The effect of labeling parameters on perfusion-based fMRI in nonhuman primates. Journal of Cerebral Blood Flow \& Metabolism 28, 640-652.

Zeng, H., and Constable, R.T. (2002). Image distortion correction in EPI: comparison of field mapping with point spread function mapping. Magn Reson Med 48, 137-146. doi: 10.1002/mrm.10200.

Zhang, W.T., Mainero, C., Kumar, A., Wiggins, C.J., Benner, T., Purdon, P.L., Bolar, D.S., Kwong, K.K., and Sorensen, A.G. (2006). Strategies for improving the detection of $\mathrm{fMRI}$ activation in trigeminal pathways with cardiac gating. Neuroimage 31, 1506-1512. doi: 10.1016/j.neuroimage.2006.02.033.

Zhao, F., Wang, P., and Kim, S.G. (2004). Cortical depth-dependent gradient-echo and spin-echo BOLD fMRI at 9.4 T. Magnetic resonance in medicine 51, 518-524.

Zilles, K., and Amunts, K. (2009). Receptor mapping: architecture of the human cerebral cortex. Current opinion in neurology 22, 331-339.

Zimmermann, J., Goebel, R., De Martino, F., Van De Moortele, P.F., Feinberg, D., Adriany, G., Chaimow, D., Shmuel, A., Ugurbil, K., and Yacoub, E. (2011). Mapping the organization of axis of motion selective features in human area MT using high-field fMRI. PLoS One 6, e28716. doi: 10.1371/journal.pone.0028716. 
Chapter 1 


\section{Chapter 2}

\section{Submillimeter $\mathbf{T}_{\mathbf{2}}$ weighted $\mathrm{fMRI}$}

\section{at $7 \mathrm{~T}$ : comparison of 3D-GRASE and 2D SE-EPI}

Corresponding publication: Kemper, Valentin G.; De Martino, Federico; Vu, An T.; Poser, Benedikt A.; Feinberg, David A.; Goebel, Rainer; Yacoub, Essa (2015). "Sub-Millimeter $\mathrm{T}_{2}$ Weighted $\mathrm{fMRI}$ at $7 \mathrm{~T}$ : Comparison of 3D-GRASE and 2D SE-EPI." Frontiers in Neuroscience 9. 


\section{Abstract}

Functional magnetic resonance imaging (fMRI) allows studying human brain function non-invasively up to the spatial resolution of cortical columns and layers. Most fMRI acquisitions rely on the blood oxygenation level dependent (BOLD) contrast employing $\mathrm{T}_{2}{ }^{*}$ weighted 2D multi-slice echo-planar imaging (EPI). At ultra-high magnetic field (i.e. $7 \mathrm{~T}$ and above), it has been shown experimentally and by simulation, that $T_{2}$ weighted acquisitions yield a signal that is spatially more specific to the site of neuronal activity at the cost of functional sensitivity. This study compared two $T_{2}$ weighted imaging sequences, inner-volume 3D Gradient-and-SpinEcho (3D-GRASE) and 2D Spin-Echo EPI (SE-EPI), with evaluation of their imaging point-spread function, functional specificity, and functional sensitivity at submillimeter resolution. Simulations and measurements of the imaging point-spread function revealed that the strongest anisotropic blurring in 3D-GRASE (along the second phase-encoding direction) was about $60 \%$ higher than the strongest anisotropic blurring in 2D SE-EPI (along the phase-encoding direction). In a visual paradigm, the BOLD sensitivity of 3D-GRASE was found to be superior due to its higher temporal signal-to-noise ratio. High resolution cortical depth profiles suggested that the contrast mechanisms are similar between the two sequences, however, 2D SE-EPI had a higher surface bias owing to the higher $\mathrm{T}_{2}{ }^{*}$ contribution of the longer in-plane EPI echo-train for full field of view compared to the reduced field of view of zoomed 3D-GRASE. 


\section{Introduction}

Since its discovery more than two decades ago (Bandettini et al., 1992; Kwong et al., 1992; Ogawa et al., 1992), non-invasive functional magnetic resonance imaging (fMRI) has evolved to become a primary method to study human brain function. The majority of $\mathrm{fMRI}$ experiments have been conducted using the blood oxygenation level dependent (BOLD) method (Ogawa et al., 1990), relying on $\mathrm{T}_{2}{ }^{*}$ changes in gradient-echo based echoplanar imaging (GE-EPI, Mansfield, 1977) acquisitions. However, it has been shown in simulations (Boxerman et al., 1995b; Uludag et al., 2009; Pflugfelder et al., 2011) and experiments at submillimeter (Duong et al., 2003; Yacoub et al., 2003; Yacoub et al., 2007) or lower resolution (Harmer et al., 2012; Boyacioglu et al., 2014; Panchuelo et al., 2015), that at high field strength (7 Tesla and above) imaging strategies utilizing $T_{2}$ weighted contrast yield higher spatial specificity than $\mathrm{T}_{2}{ }^{*}$ weighted contrast. This becomes particularly relevant when the investigated functional organization is on the order of $1 \mathrm{~mm}$ or smaller, requiring avoidance of pial vein effects that have been shown to be stronger in $\mathrm{T}_{2}{ }^{*}$ weighted acquisitions (Yacoub et al., 2005). Cerebral blood flow or blood volume based approaches present an alternative approach to high-resolution fMRI (see e.g. Jin and Kim, 2008a; Goense et al., 2012; Huber et al., 2015a).

By far, most $T_{2}$ weighted fMRI experiments have been conducted using $2 \mathrm{D}$ spin-echo EPI sequences ((Bandettini et al., 1994), see (Norris, 2012) for review). The EPI echo train increases the temporal and the specific absorption rate (SAR) efficiency as compared to purely $T_{2}$ weighted sequences such as RARE/FSE (Hennig et al., 1986; Constable et al., 1994; Poser and Norris, 2007) or to balanced and non-balanced steady-state free precession sequences (b-SSFP/nb-SSFP) (Auerbach et al., 2002; Bieri and Scheffler, 2007; Miller and Jezzard, 2008; Barth et al., 2010; Goa et al., 2014). However, the EPI echo train causes blurring in the phase-encoding direction due to $T_{2}{ }^{\prime}$ weighting (Constable and Gore, 1992) and increases the $\mathrm{T}_{2}{ }^{*}$ weighting as demonstrated by Goense et al. 2006.

As an alternative, inner-volume excitation approaches, such as 3D Gradient-and-Spin-Echo (3D-GRASE) (Oshio and Feinberg, 1991; Feinberg et al., 2008) or single slice inner volume 2D-SE EPI (Duong et al., 2002; Yacoub et al., 2003) have been proposed for $T_{2}$ weighted submillimeter $\mathrm{fMRI}$ in humans. The 3D-GRASE sequence combines EPI- 
readouts with fast spin-echo acquisition schemes (RARE/TSE) by acquiring multiple EPI readouts separated by refocusing pulses. Several secondary phase-encoding steps (referred to as "partitions" throughout this manuscript) are acquired after a single excitation pulse thus overcoming the single slice limitation of zoomed inner volume EPI. Slab-selective refocusing pulses are applied perpendicularly to the slab-selective excitation pulse to limit the required phase-encoding steps (Feinberg et al., 2008). This inner-volume selection allows for shorter EPI echo-trains, minimizing both $\mathrm{T}_{2}{ }^{*}$ contrast and blurring in the in-plane phase-encoding direction. At the same time, short EPI echo-trains keep the overall echo train length short to minimize blurring along the partition direction related to $\mathrm{T}_{2}$ decay. Centric ordering of the partitions is typically chosen to sample k-space center at maximal SNR (minimal TE). Compared to 2D spin-echo EPI sequences, 3D-GRASE offers a potential SAR advantage because it uses fewer radiofrequency (RF) pulses due to the necessity of only one excitation pulse for the entire $3 D$ volume and a reduced number of refocusing pulses due to partial Fourier acquisitions along the partition direction. The parsimony in SAR can be used to improve the excitation and refocusing slab profiles by increasing the bandwidth-time product of the RF pulses or to shorten the RF pulse duration in order to shorten the overall echo train length. Cortical depth-specific and columnar fMRI studies have been successfully conducted using 3D-GRASE (Zimmermann et al., 2011; Olman et al., 2012), and it has been shown that 3D-GRASE yields a more specific signal than GE-EPI (De Martino et al., 2013). However, thus far, a systematic comparison of 3D-GRASE and 2D SE-EPI has been missing.

Therefore, this study compares 3D-GRASE and 2D SE-EPI for submillimeter resolution $\mathrm{fMRI}$ experiments at $7 \mathrm{~T}$. We do so by simulating and measuring the imaging point-spread function (referred as to PSF through this manuscript) reflecting the anisotropic spread of information in the image stemming from an idealized point source. The point-spread function is defined here as the magnitude of the Fourier transform of the complex optical transfer function, a function reflecting a modulation of k-space data (i.e. truncation associated with the finite nominal image resolution and signal reduction by relaxation weighting at different positions in k-space), whose magnitude is the so-called modulation transfer function (MTF) (Robson et al., 1997). $\mathrm{T}_{2}$ or $\mathrm{T}_{2}{ }^{*}$ weighting along an echo-train and truncation of $k$-space thus impose limits on the effective resolution of the image. 
Further, we measure temporal stability (temporal signal-to-noise ratio, tSNR), functional responses to visual stimulation, and the cortical depth profiles of the two acquisitions.

\section{Methods}

All measurements were performed on a 7T Siemens Magnetom scanner equipped with a body gradient system $(70 \mathrm{mT} / \mathrm{m}$ maximum amplitude, $200 \mathrm{mT} / \mathrm{m} / \mathrm{s}$ maximum slew rate) and a dedicated surface coil (16-channel receive and a separate 4 channel transmit coil with a pre-determined static $B_{1}$ phase set optimized for homogeneity over the visual cortex; Live Services, Minneapolis, MN, USA) covering the posterior part of the subjects' heads. Compared to a whole brain coil, this RF coil offers better transmit efficiency (reduced SAR) in occipital areas and a wide angle for visual stimulation. Three subjects (two females, age $(28.3 \pm 1.5)$ years) were scanned for imaging point-spread function (PSF) and functional measurements. Three additional male subjects (age $33.3 \pm 3.5$ ) were scanned for the PSF measurements only (see below). All subjects were scanned after giving informed written consent and in compliance with the local ethical board.

\section{Visual stimulation protocol}

Functional data were acquired while the subjects were performing a visual stimulation task. High contrast concentric flickering checkerboard patterns were displayed centrically on a screen in the magnet bore with a visual angle of 39 degrees. A block design was chosen with 14 blocks of 10 seconds stimulation and 12 seconds rest. The subjects were instructed to fixate a point in the middle of the screen.

\section{Simulations}

The signal evolution throughout the echo trains of 3D-GRASE and 2D SE-EPI were simulated in MATLAB (The MATHWORKS Inc., Natick, MA, USA). The same imaging parameters were used in the PSF measurements as in the functional experiments (see below). For the 2D SE-EPI, the signal modulation transfer function (MTF, Robson et al., 1997) was modeled as an exponential decay with a time constant $\left(T_{2}\right)$ of $50 \mathrm{~ms}$ (see Uludag et al., 2009, for review of relaxation times) in addition to an exponential decay with a time constant $\left(T_{2}{ }^{\prime}\right)$ of $63 \mathrm{~ms}$ (where $T_{2}{ }^{*}=1 /\left(1 / T_{2}+1 / T_{2}{ }^{\prime}\right)=27.8 \mathrm{~ms}$ ) 
symmetrically on both sides of the central spin-echo (Bernstein et al., 2004), such that the outer k-space lines experience a stronger modulation. To account for partial Fourier acquisitions (Feinberg et al., 1986), the missing k-space lines were zero-filled. Accelerated imaging was accounted for by assuming the acquisition of missing k-space lines interspersed equidistantly between the acquired lines such that the total echo-train duration was maintained.

The in-plane phase-encoding part of the 3D-GRASE sequence was simulated as in the 2D SE-EPI case, whereas the partition direction was simulated to experience an exponential decay with the same time constant $\left(T_{2}=50 \mathrm{~ms}\right.$, equivalent to perfect $180^{\circ}$ refocusing pulses). Centric reordering was taken into account and the same procedure to mimic partial Fourier acquisition was applied.

In the model it was assumed that the modulation along the phaseencoding direction is separable from the modulation along the partition direction in 3D-GRASE. In order to obtain the simulated PSF at a $1 \mu \mathrm{m}$ resolution the simulated MTF was Fourier transformed after zero-filling to account for the truncated k-space acquisition. This simulation was repeated for the phase-encoding direction of both sequences and the partition direction of 3D-GRASE. All parameter sets used in the experiments were considered. A normal distribution was fitted to the magnitude of each PSF using a linear least squares regression with four free parameters, amplitude, offset, standard deviation and baseline. A Gaussian was chosen because it well approximated the shape of the central lobe of the PSF (i.e. the convolution of the decay filter and a sinc-kernel due to the k-space truncation) and it allows to easily parameterize the full width at half maximum (FWHM).

\section{Point-spread function measurements}

PSF measurements were performed with the same imaging parameters as the functional measurements (see below) and the simulations, except for the number of measurements and a lower image scaling factor to adjust the dynamic range to accommodate the high image intensity in the k-space center. The vendor's product 2D SE-EPI sequence was used after implementing the option to turn off the phase-encoding gradients. Further, the gradient polarity of the refocussing gradient was inverted to allow lowSAR fat suppression (Park et al., 1987; Volk et al., 1987; Gomori et al., 1988; 
Ivanov et al., 2010) in the PSF measurements and the functional measurements.

Four volumes were acquired to reach a steady state. In the fifth repetition, the phase-encoding gradients were switched off, so that the k-space center was acquired in every readout line. This procedure yields non-Fourierencoded images (Fourier-encoded only along the readout direction), which are processed in the image reconstruction pipeline nonetheless. Since the acquired data does not contain any spatial information, but does experience the MTF (i.e. the weighting due $T_{2}$ and $T_{2}{ }^{*}$ decay), the resulting image reflects the point-spread function due to the acquisition and reconstruction (Mugler et al., 1992; Robson et al., 1997; Zaitsev et al., 2004; Park et al., 2007). In the ideal case with no signal decay, all acquisition lines would be identical leading to a reconstructed image with a delta-function like intensity distribution along the non-encoded directions. However, in practice, due to the non-uniformity of the MTF, the peak has a finite width and exhibits side lobes in the case of partial Fourier acquisitions.

The data were analyzed as follows. After standard image reconstruction, individual lines (in phase-encoding or partition encoding direction) were admitted to the analysis if their maximum pixel value was at least $25 \%$ of the global image intensity to avoid noise-only lines. These lines were centered on their respective maximums. Then, data were interpolated using a spline interpolation and a normal distribution was fitted as to the simulated data described above. In the 2D SE-EPI acquisitions, as many peaks as the GRAPPA acceleration factor appear in the data due to the aliasing, but only the maximum peak was used for fitting. This makes the results more comparable to the 3D-GRASE data and reduces an overestimation of the PSF due to the collapsing of the PSF over the entire field of view (FoV, see discussion for details).

In order to assess the slice profile of 2D SE-EPI (i.e. the point spread function along the slice direction), we simulated profiles of the used excitation and refocusing pulses by Fourier transforming the vendorprovided RF pulse shapes. Perfect $90^{\circ}$ and $180^{\circ}$ pulses were assumed the slice center. The resulting slice profiles were calculated as $\sin \left(\alpha_{1}\right) \times$ $\sin ^{2}\left(\alpha_{2} / 2\right)$, where $\alpha_{1}$ and $\alpha_{2}$ denote the spatially dependent excitation and refocusing angles, respectively. The final slice profile was obtained by 
assuming saturation recovery from the excitation pulse during one TR at the original slice location and during half a TR at the location of the neighboring slices (approximating interleaved slice acquisition). Saturation recovery from both a fully relaxed system (longitudinal magnetization $\mathrm{mz}=1$ ) and an Ernst steady state were considered in a range of $\mathrm{T}_{1}$ values between $1200 \mathrm{~ms}$ and $1800 \mathrm{~ms}$ (step size of $100 \mathrm{~ms}$ ).

\section{Functional measurements}

Five different acquisitions were repeated two times in each scanning session in a pseudo-random order. All acquisitions had a nominal isotropic resolution of $(0.8 \mathrm{~mm})^{3}$ of and repetition time (TR) of $2000 \mathrm{~ms}$. All used nominal excitation/refocussing flip angles of $90^{\circ} / 180^{\circ}$. Additional acquisition parameters are listed in Table 2-1. 3D-GRASE 2 is identical to 3D-GRASE 1 except the image volume was rotated about the read-direction such that the phase-encoding direction of 3D-GRASE 1 was the same as partition encoding direction of 3D-GRASE 2 and vice versa. The slices in all measurements except 3D-GRASE 2 were placed parallel to calcarine sulcus (anterior-posterior (ant-pos)), and orthogonally to that in 3D-GRASE 2 (inferior-superior (inf-sup)). Phase-encoding direction left-right was chosen for all 2D SE-EPI acquisitions to keep the FoV to a minimum, while avoiding aliasing in the region of interest. Although the echo spacing of the 2D SEEPI acquisitions had to be slightly increased with respect to 3D-GRASE to avoid critical peripheral nerve stimulation, phase-encoding direction leftright allowed much shorter EPI echo train durations than anterior-posterior in 2D SE-EPI.

Automatic second order shimming routines were used to homogenize the static magnetic field $\left(B_{0}\right)$ in a region of interest big enough to accommodate the 2D SE-EPI FoV. The shim was adjusted manually for further improvements of the homogeneity, until the FWHM of the measured Larmor frequencies was well below $40 \mathrm{~Hz}$. Macroscopic inhomogeneities further cause a reduction in the apparent $\mathrm{T}_{2}{ }^{*}$. We obtained a macroscopic, volumetric $\mathrm{T}_{2}{ }^{*}$ of $17 \pm 2 \mathrm{~ms}$ (mean $\pm \mathrm{SD}$ across all six participants). The individual subjects' volumetric $\mathrm{T}_{2}{ }^{*}$ values were also used in the PSF simulations in addition to the theoretical $\mathrm{T}_{2}{ }^{*}$ values for cortical gray matter. A constant shim was then used throughout all functional and PSF measurements. The transmit field $\left(\mathrm{B}_{1}^{+}\right)$was measured using a pre-saturation based $B_{1}$ mapping sequence provided by the scanner 
vendor. The transmit voltage was adjusted to yield the correct flip angles in the approximate location of the calcarine sulcus.

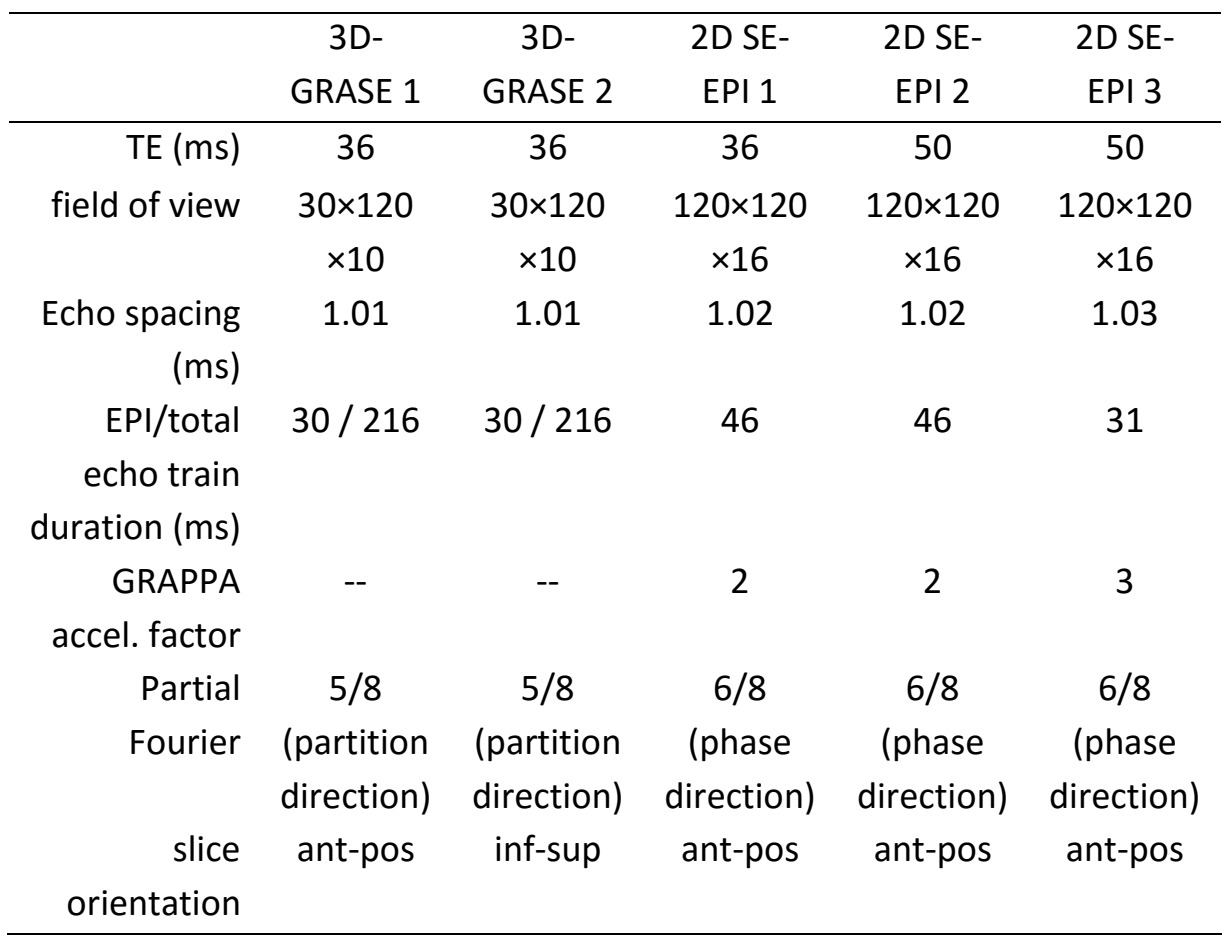

Table 2-1: Acquisition parameters of the five functional imaging protocols

\section{Anatomical measurements}

Structural images were acquired for anatomical reference using a $\mathrm{T}_{1}$ weighted MPRAGE sequence (isotropic resolution of $0.6 \mathrm{~mm}$; $\mathrm{TR}=3100 \mathrm{~ms} ; \mathrm{TI}=1500 \mathrm{~ms} ; \mathrm{TE}=2.52 \mathrm{~ms} ;$ flip angle = 5 degree; GRAPPA acceleration factor $=3 ; \quad F o V=230 \times 230 \times 154 \mathrm{~mm}^{3}$ ). Additional proton density weighted images were acquired using identical imaging parameters (except $T R=1440 \mathrm{~ms}$ ) without the inversion module. These additional images were used to alleviate inhomogeneities across the FoV in postprocessing by dividing the $T_{1}$ weighted image pixel values by those of the proton density weighted images (Van de Moortele et al., 2009). 


\section{Functional data analysis}

Data analysis was performed using BrainVoyager QX 2.8 (Brain Innovation, Maastricht, The Netherlands) and custom-written routines in MATLAB. Standard preprocessing of the $\mathrm{FMRI}$ data consisted of 3D rigid body motion correction and temporal high-pass filtering by a General Linear Model (GLM) with three cosine cycles and a linear trend regressor for each run. The average motion parameters for all acquisitions were $0.32 \pm 0.31 \mathrm{~mm}$ translation and $0.31 \pm 0.28$ degree rotation (mean \pm SD of absolute deviation of both parameters). No difference between 3D-GRASE and 2D SE-EPI acquisitions was observed. SE-EPI data was additionally slice-time corrected using a sinc-weighted interpolation. These data were then coregistered with the structural images using positional information of the acquisitions. Manual adjustments were performed using edge information in the $\mathrm{fMRI}$ data. For this purpose, the fMRI data was averaged across time after preprocessing to yield a single high quality image, with fine-grained structural information (i.e. CSF/gray matter and white matter/gray matter boundaries), for alignment. When image distortions were too strong to guarantee good alignment of the entire volume, multiple alignments were performed specifically in order to optimize the co-registration for the targeted regions, in which cortical depth profiles were generated. Finally, functional images were resampled in the $3 D$ anatomical volume at a resolution of $0.8 \mathrm{~mm}$ using a sinc interpolation.

A standard GLM was used to assess the functional activation. The visual stimulation predictor was created by convolving the visual activation blocks with a standard hemodynamic response function (HRF). For each imaging sequence, both runs were analyzed together on a single subject level without additional confound predictors.

Temporal Signal-to-Noise (tSNR) maps were created by dividing the voxelwise temporal mean intensity by the temporal standard deviation. To produce tSNR maps, preprocessing included only high-pass filtering, using four cosine cycles and linear trend removal. No motion correction or slicetime correction was applied to avoid additional spatial interpolation. The effect of the visual stimulation was removed from the voxels' time course using univariate regression (i.e. GLM).

For the cortical depth profile analysis, high resolution cortical depth grids were created similar to as described in De Martino et al. (2013) based on 
anatomical data after segmenting cortical gray and white matter and delineating the boundary between gray matter and cerebrospinal fluid (CSF). The automatic segmentation in BrainVoyager QX was used and refined manually in the regions of interest to ensure correct classification of white matter, gray matter and other tissue types. In each region of interest, a stack of five cortical grids were created starting from cortical thickness measurements (Jones et al., 2000). Grids were placed at regular intervals of cortical depth, and neighboring sampling points within a grid were regularly spaced. A single grid would sample $8 \times 8 \mathrm{~mm}^{2}$ on a flat piece of cortex. A total of 10 regions of interest were defined within the foldings of the calcarine sulcus. Figure 2-1 displays an exemplary cortical depth sampling region of interest.

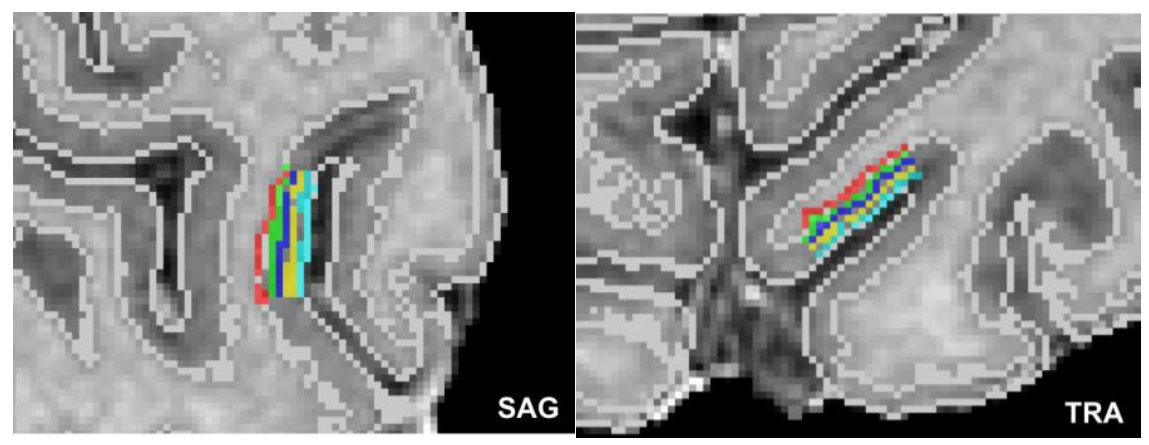

Figure 2-1: Exemplary high resolution cortical grid sampling prescription. Cortical depth dependent grids were prescribed at $10 \%$ (red), $30 \%$ (green), $50 \%$ (dark blue), $70 \%$ (yellow), and $90 \%$ (light blue) cortical depth as defined between the white matter-gray matter boundary and the gram matter-CSF boundary (light gray lines), as segmented based on the anatomical reference images.

After the creation of relative cortical depth grids, the local cortex normal vectors (the vectors orthogonal to the cortical sheet) were approximated linearly by calculating the difference vector between the grid points at $10 \%$ cortical depth and the corresponding grid points at $90 \%$ cortical depth. The angle between these cortex normal vectors and the orientation of the partition directions of both 3D-GRASE 1 and 3D-GRASE 2 were calculated. 


\section{Results}

\section{Imaging point-spread function simulations and measurements}

Figure 2-2 shows the results of the simulations and the imaging PSF measurement for all acquisitions, demonstrating good agreement between experimental data and simulations for both the MTF (top) and the PSF (bottom). Prior to the creation of the MTFs, the raw data from the PSF measurements were coil-combined (sum-of-squares combination). The maximum of each readout line (i.e. the echo) was selected and averaged across the other imaging dimension (slices/partition direction for phaseencoding direction; phase-encoding direction for partition direction), and normalized in the range $[0,1]$. Note the asymmetry in MTF with higher intensities in the echoes before than after the central spin-echo as expected from combined $T_{2}$ and $T_{2}{ }^{\prime}$ modulation. This signal characteristic is often neglected in over-simplified models assuming only a symmetric $\mathrm{T}_{2}{ }^{*}$ signal decay around the spin-echo.

\begin{tabular}{|c|c|c|c|c|}
\hline \multirow[b]{2}{*}{ Acquisition } & \multirow[b]{2}{*}{ Direction } & \multicolumn{3}{|c|}{ FWHM (pixel) } \\
\hline & & Measurement & $\begin{array}{c}\text { Simulation } \\
\mathrm{GM} \mathrm{T}_{2}{ }^{*}\end{array}$ & $\begin{array}{c}\text { Simulation } \\
\text { Exp. } \mathrm{T}_{2}{ }^{*}\end{array}$ \\
\hline 3D-GRASE 1 & $\mathrm{PE}$ & $1.8 \pm 0.3$ & 1.2 & $1.3 \pm 0.0$ \\
\hline 2D SE-EPI 1 & PE & $2.3 \pm 0.2$ & 2.0 & $2.3 \pm 0.1$ \\
\hline 2D SE-EPI 2 & PE & $2.2 \pm 0.1$ & 2.0 & $2.3 \pm 0.1$ \\
\hline 2D SE-EPI 3 & PE & $2.0 \pm 0.1$ & 1.8 & $2.0 \pm 0.1$ \\
\hline 3D-GRASE 1 & partition & $3.6 \pm 0.5$ & 4.4 & \\
\hline
\end{tabular}

Table 2-2: FWHM of the PSFs of the different acquisitions (mean \pm standard deviation).

Table 2-2 reports the mean across lines and subjects of the full width half maximum of the PSF (mean \pm standard deviation). The FWHM of the partition direction in 3D-GRASE 1 was found to be $3.6 \pm 0.5$ pixels, which is about $56 \%$ and $63 \%$ higher than the FWHMs of the phase-encoding direction of 2D SE-EPI 1 and 2. Phase-encoding direction of 2D SE-EPI 3 and 3D-GRASE 1 acquisitions have smaller FWHMs due to the reduced number of phase-encoding steps. The experimental findings are in good agreement with the simulations. The agreement is enhanced when using the experimentally obtained volumetric $\mathrm{T}_{2}{ }^{*}$ values (right column) in the simulation rather than literature values for gray matter (middle column). 


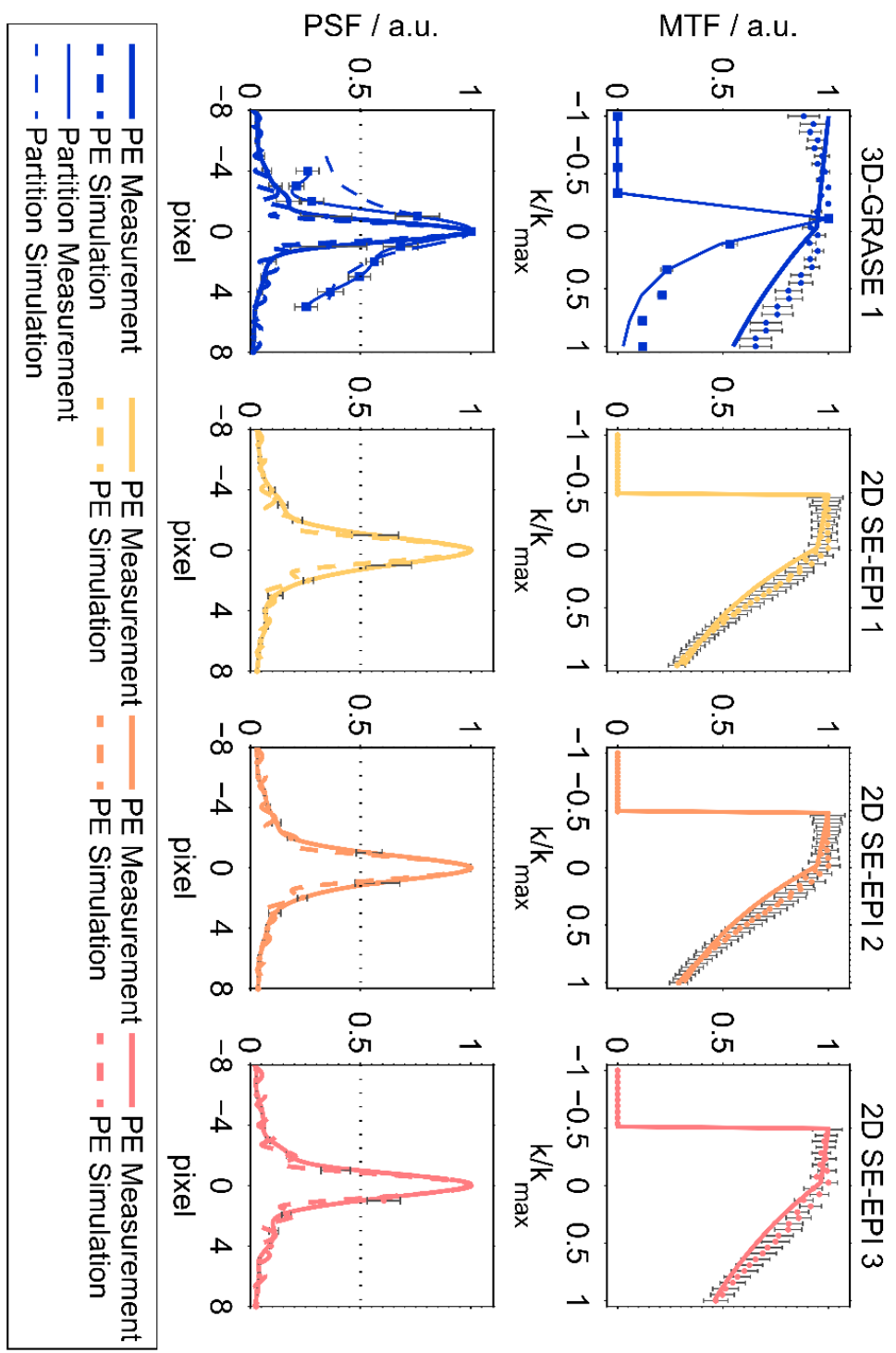

Figure 2-2: Averaged modulation transfer functions (MTFs, top) and pointspread functions (PSFs, bottom). The top panel shows averaged MTFs in phase-encoding direction (thick lines) and partition encoding direction for 3D-GRASE sequences (thin lines). Solid lines represent averaged experimental results across all subjects, dashed lines represent simulations. The dimension of the horizontal axis in the top panel is relative k-space. In the bottom panel, the dimension of the horizontal axis is pixels in absolute numbers (cropped to center) for better comparison. Error bars indicate standard deviation across subjects. 

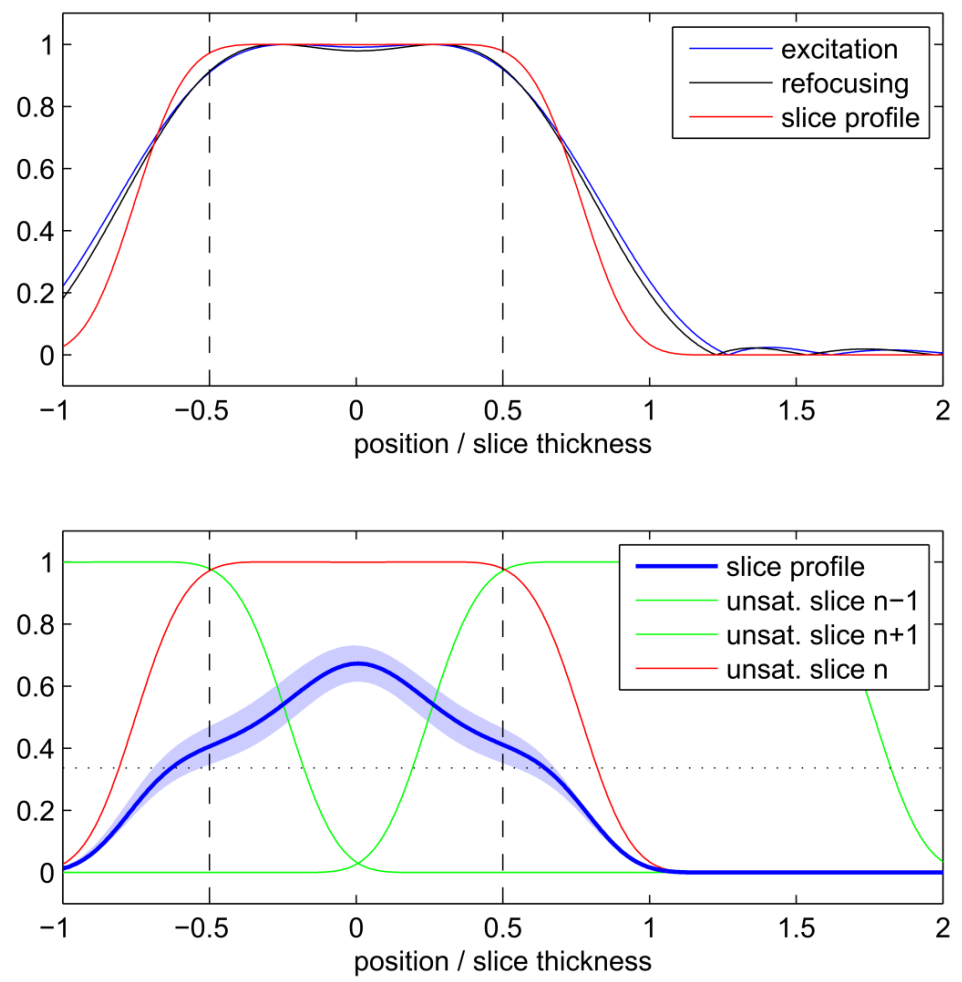

Figure 2-3: Simulated 2D SE-EPI slice profile of a single slice (top) and with consideration of neighboring slices and saturation recovery (bottom). Profiles are displayed relative to nominal slice thickness.

The simulation results of the 2D SE-EPI slice profile are displayed in Figure 2-3. The FWHM (indicated by the vertical dashed line in the figure) was found to be $1.3 \pm 0.2$ times the nominal slice thickness (mean $\pm S D$ across different $T_{1}$ values and saturation scenarios). Due to the wide saturation bands of neighboring slices, the effective slice profile resembles a triangle rather than the desired rectangle. When taking into account the saturation bands of neighboring slices, the integral of the attainable signal is reduced by $49 \pm 5$ percent compared to a single slice without $T_{1}$ saturation effects and by $35 \pm 5$ percent compared to a single slice with $\mathrm{T}_{1}$ saturation effects. 


\section{Temporal signal-to-noise ratio}

Figure 2-4 displays central slices of the tSNR maps and full volume tSNR histograms. The 2D SE-EPI data were cropped to the same in-plane FoV as the 3D-GRASE 1 acquisition (disregarding increased image distortions in 2D SE-EPI). The images and histograms show that the tSNR is substantially higher in 3D-GRASE than in all 2D SE-EPI acquisitions. 3D-GRASE 2 yields similar tSNR as 3D-GRASE 1 (not shown because of its different slice orientation). The $2 D$ SE-EPI 1,2 , and 3 (short TE $(R=2)$, long TE $(R=2)$, long TE $(R=3)$ ) yield tSNR in descending order as expected due to $T_{2}$ relaxation, undersampling $(R)$, and noise amplification (g-factor) from higher acceleration. Similar results were found for all other subjects (not shown).

\section{$\underline{\text { Visual activation }}$}

All functional acquisitions elicited significant responses to the visual stimulation in and around the calcarine sulcus. Figure 2-5 displays results of the functional experiment overlaid on both an anatomical reference (sagittal cut, left), and on averaged functional slices (single central slice). The same statistical threshold was chosen for all acquisitions, and no multiple comparison correction was used in order not to favor the reduced FoV acquisitions. Note the absence of activated voxels in white matter regions in all acquisitions. BOLD sensitivity is significantly lower in the 2D SE-EPI acquisitions as a consequence of the reduced tSNR (see Figure 24).

Figure 2-6 displays histograms of the BOLD signal changes (top) and tvalues (bottom) for all three subjects. For each acquisition, voxels were included in the analysis if they responded significantly $(p<0.05$ uncorrected). Only the mutually covered region was considered. Border voxels were discarded to exclude artefacts from motion or slow scanner drift. The histograms reveal that in each subject, smaller BOLD signal changes surpass the significance threshold using 3D-GRASE, indicative of smaller variability in the responses. In consequence, more active voxels are detected in 3D-GRASE 1 than in any of the 2D SE-EPI acquisitions (see Figure 2-6 bottom). 

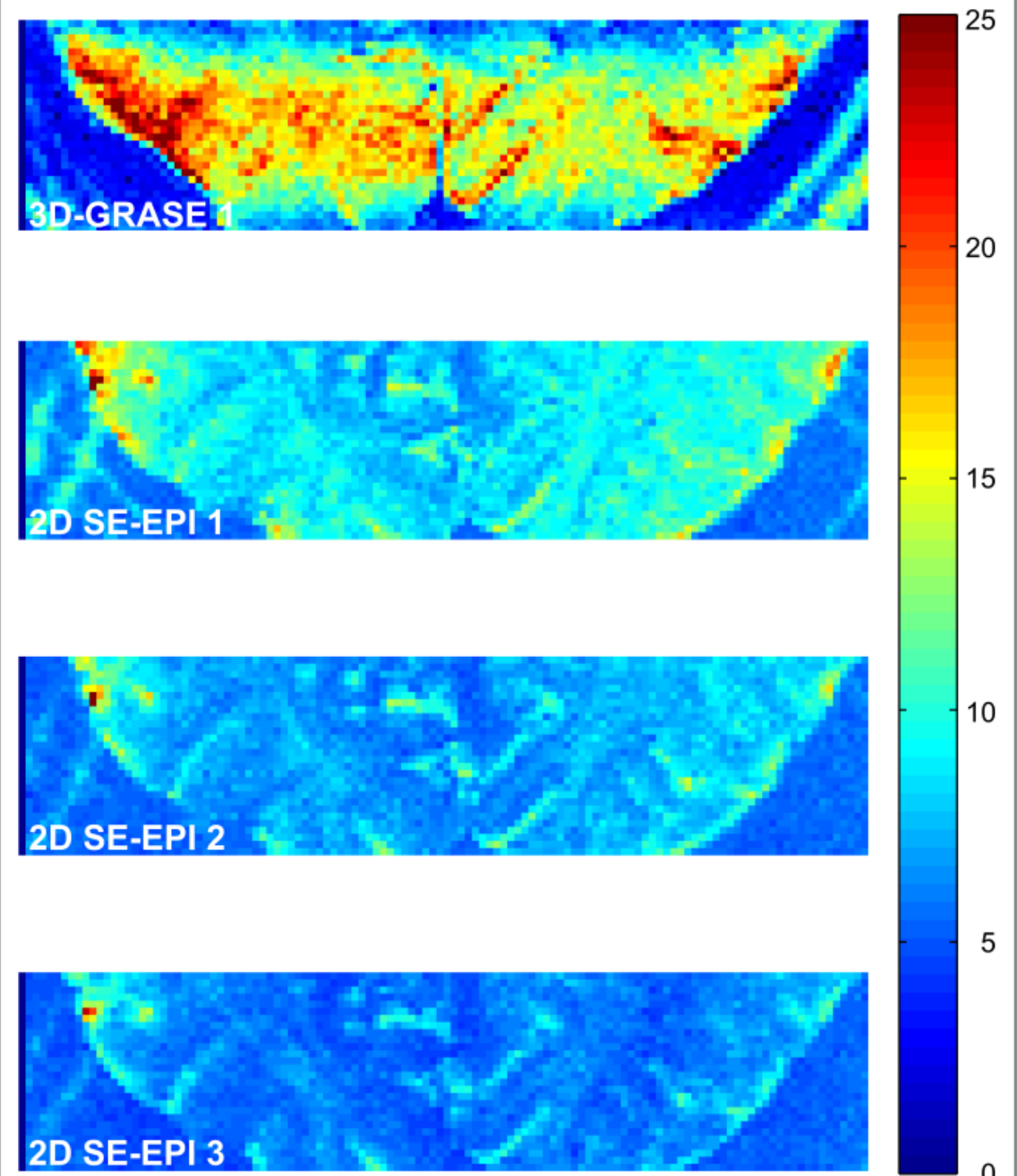

Figure 2-4 (double page): Temporal signal-to-noise-ratio maps. Maps of the five different acquisitions in a single subject (left) and corresponding histograms (right) are displayed. The 2D SE-EPI images are cropped to the same FoV as 3D-GRASE 1. All maps have the same color scale. Histograms display the tSNR distribution of the same in-plane FoV in all 3D-GRASE slices and 2D SE-EPI slices corresponding to 3D-GRASE 1. 


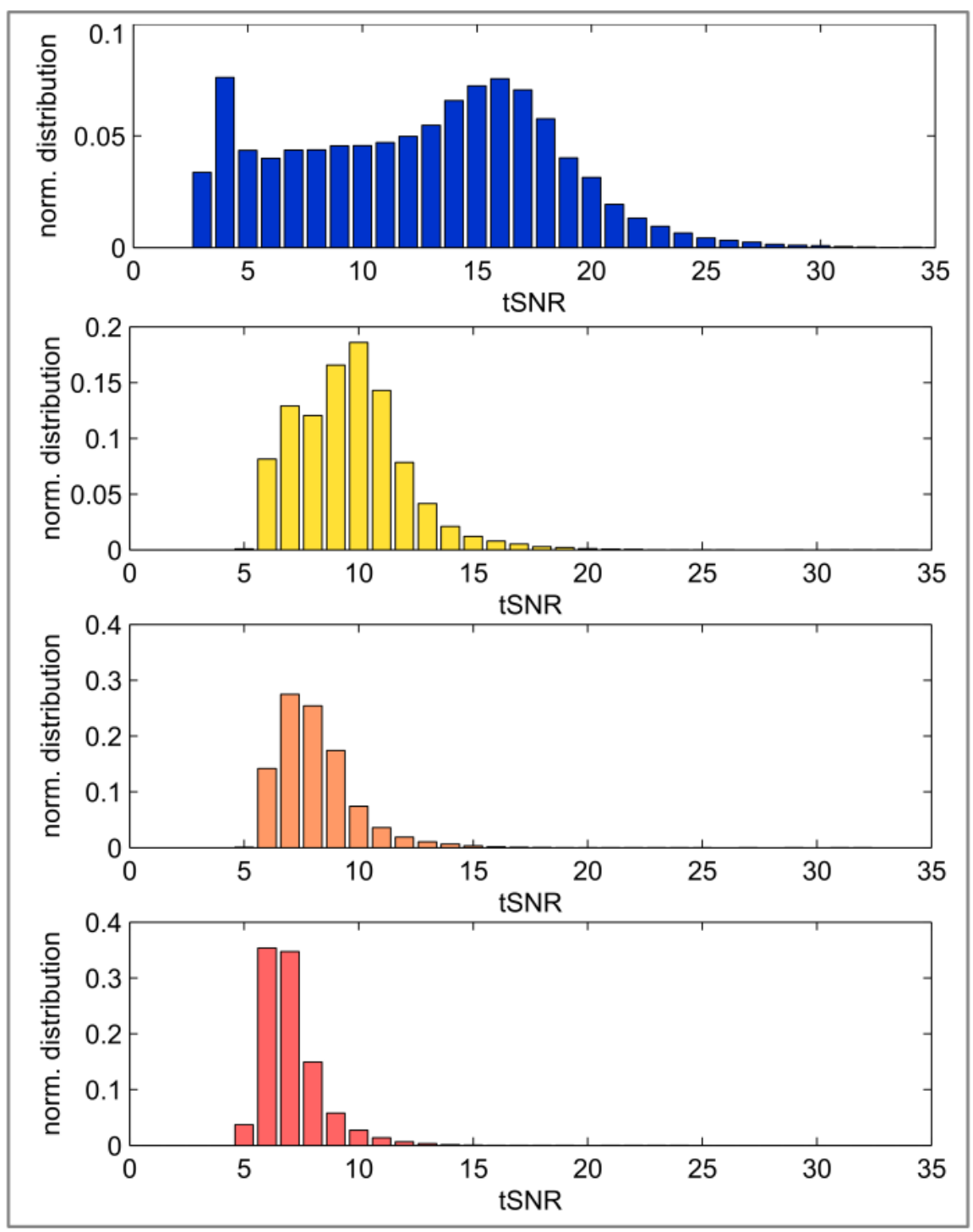



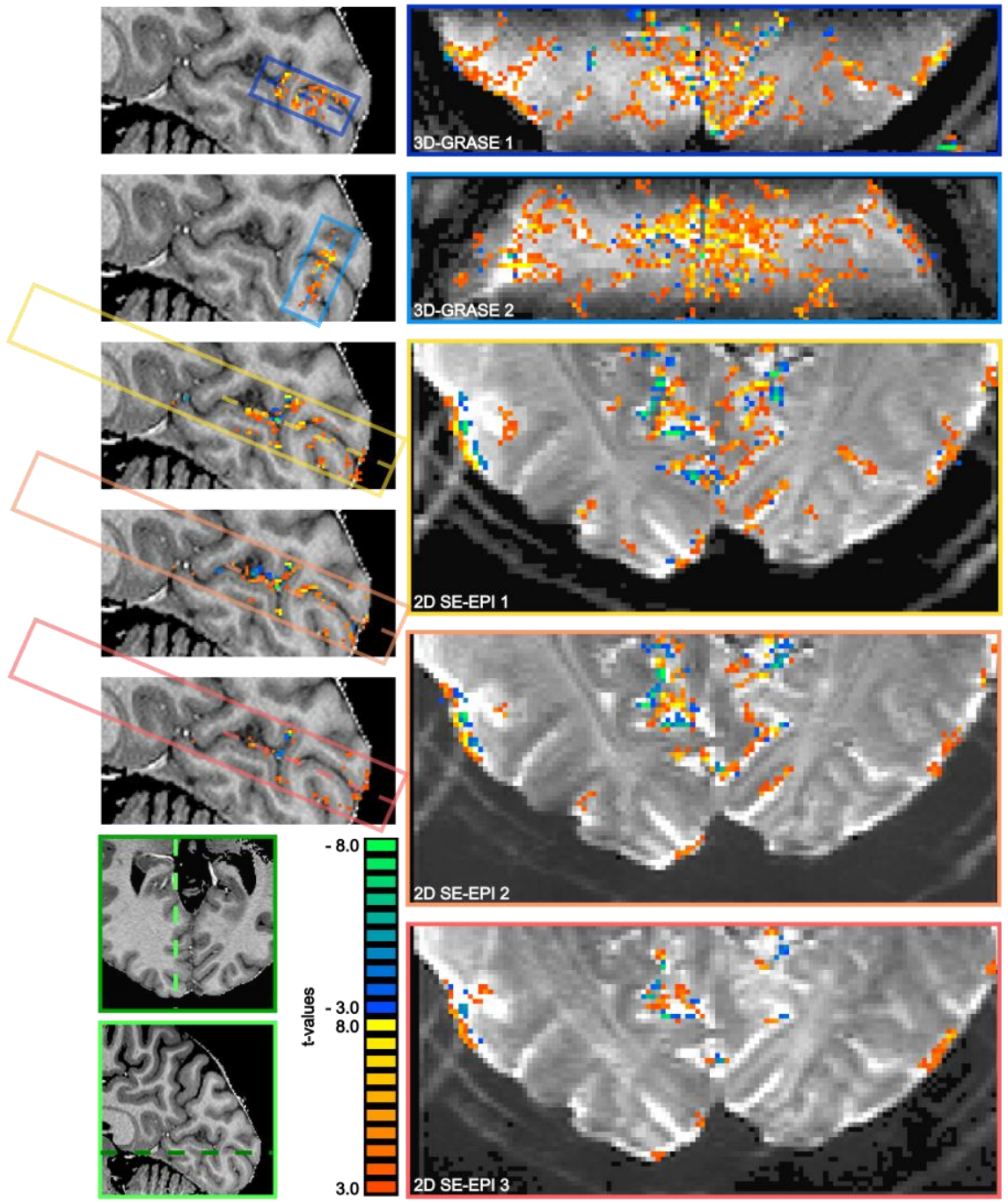

Figure 2-5: BOLD responses overlaid on anatomical reference and averaged functional slices. Mid-sagittal slices of the reference anatomical scan (left) and central functional slices (right) are shown, as indicated by the dashed lines. Note the oblique coronal orientation of 3D-GRASE 2. The same statistical threshold was applied to all acquisitions ( $t>3.0, p<0.003$ twosided t-test on single voxel level, cluster threshold of 4 voxels). The position of the sagittal cut is shown on a transversal slice in the bottom left corner. The extent and orientation of the functional slices as well as the displayed slice position (dashed lines) are indicated by the colored boxes in the sagittal images. 

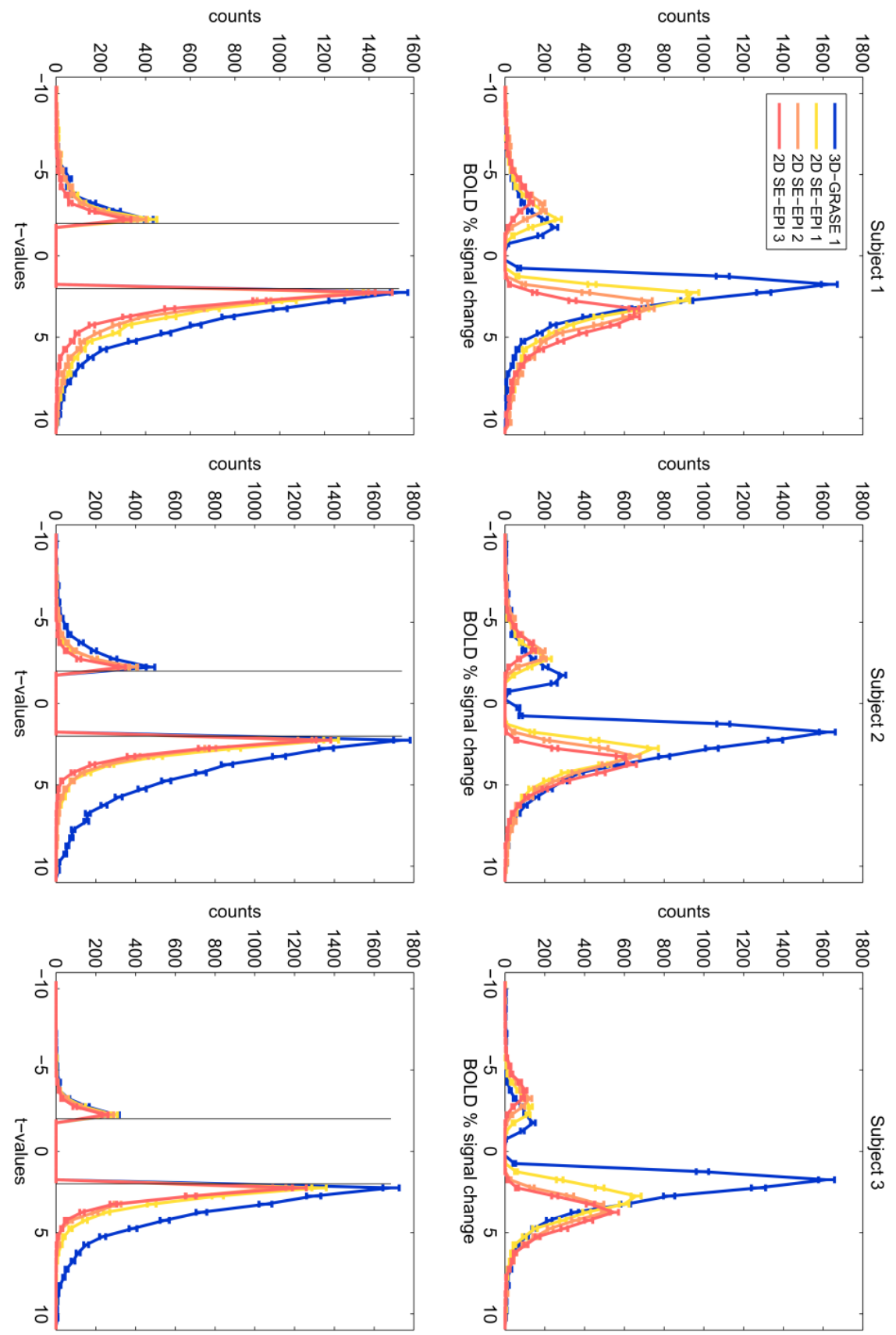

Figure 2-6: Histograms of significant voxels. Counts of BOLD signal changes (top) and t-values are shown for voxels, which responded significantly to the stimulation $(t>2.0, p<0.046)$. 


\section{Cortical depth profiles}

Figure 2-7 displays cortical depth profiles from individual regions and their average from 3D-GRASE 1, 2D SE-EPI 1 and 2D SE-EPI 3. 2D SE-EPI 2 was similar to 2D SE-EPI 1 and 2D-SE-EPI 3 (not shown for clarity). Grid points were included in the spatial average, if at least one of the three displayed acquisitions elicited significant responses ( $p<0.05$, uncorrected) in at least one of the five cortical depths. The responses measured with these protocols are similar showing BOLD signal changes mostly between $1 \%$ and $3 \%$. In both 2D SE-EPI and 3D-GRASE the BOLD signal change is significantly higher at $50 \%$ cortical depth than at $10 \%$ (two-sided Wilcoxon signed-rank test, $p<0.02$, uncorrected). The 2D SE-EPI acquisitions increase further towards the pial surface (signal change is different between $50 \%$ and $90 \%$ at $p<0.02$, two-sided Wilcoxon signed-rank test, for 2D SE-EPI 1 and 2, not significant for 2D SE-EPI 3), whereas the 3D-GRASE profile shows a trend to decrease towards the surface.

In order to assess the potential effect of the imaging PSF on the cortical depth profiles dependent profiles, we repeated the cortical depth profile analysis after smoothing the 2D SE-EPI 1 data using an anisotropic Gaussian kernel. We used two different sizes of the smoothing kernel (FWHM of 2.4 and 4.7 pixels) in slice direction (no additional smoothing in other directions) a range that includes the estimated FWHM of 3D-GRASE 1 in the partition direction. The comparison of normal and blurred data is shown in Figure 2-8. The noted increase towards the pial surface in 2D SE EPI remains despite the smoothing. All profiles differ significantly between $10 \%$ and $90 \%$ cortical depth (two-sided Wilcoxon signed-rank test, $p<0.002$, uncorrected).

We also evaluated the difference between 3D-GRASE 1 and the orthogonally oriented 3D-GRASE 2 in four of the individual regions which were sufficiently covered by both acquisitions (ROIs 2, 5, 7, and 10 in Figure 2-7). Histograms of the angle (limited between $0^{\circ}$ and $90^{\circ}$ ) between the cortex normal vectors and the partition directions of both 3D-GRASE 1 and 2, are presented at the top of the Figure 2-9. The averages of the cortical depth profiles in these regions are displayed at the bottom in the same figure. Despite the difference in acquisition orientation the depth dependent functional profiles are similar to one another, and are similar to 
the 3D-GRASE 1 profile in Figure 2-9 although only a subset of ROIs was used.
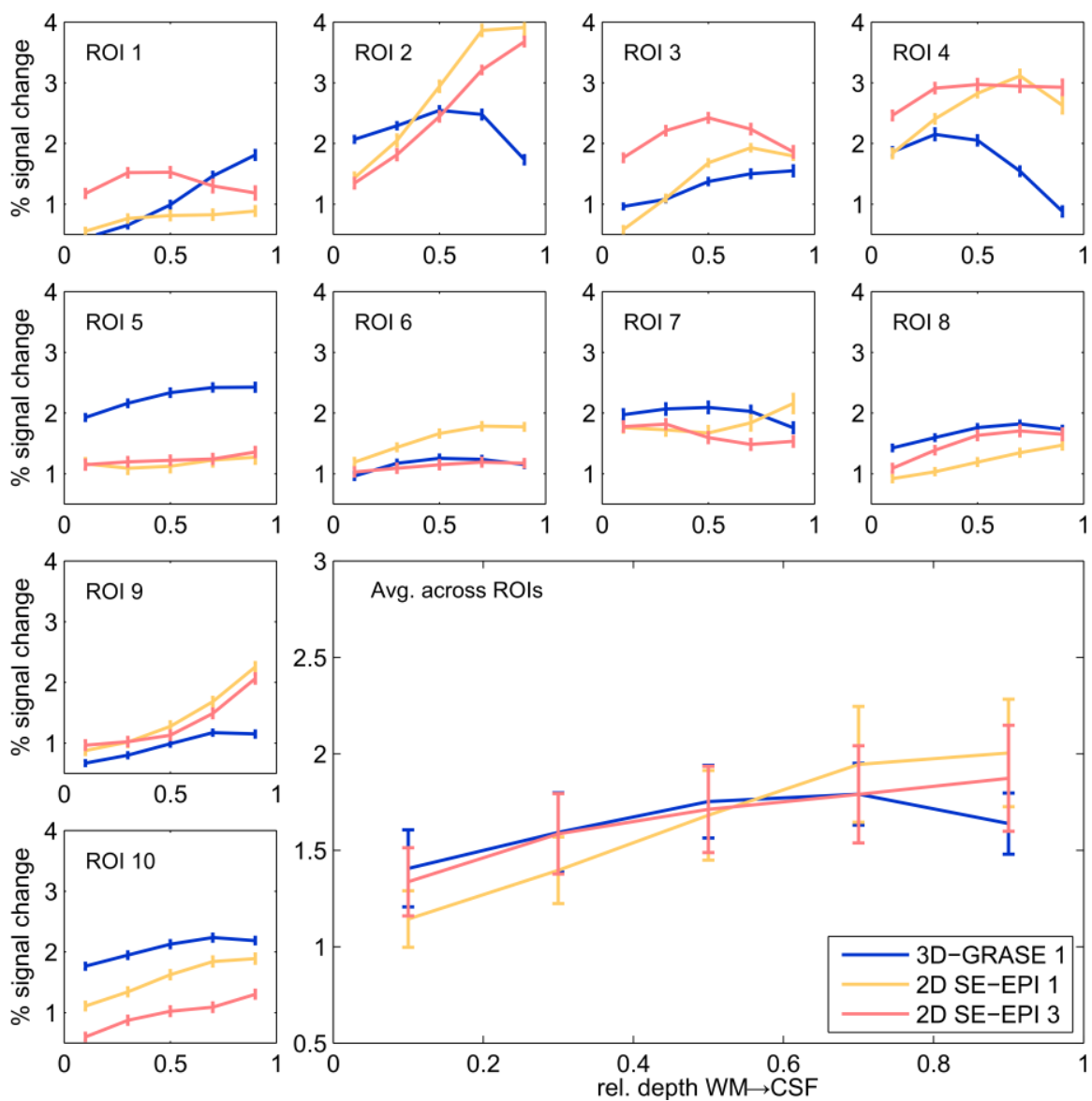

Figure 2-7: Cortical depth profiles in individual regions. Single regions (small graphs) and their average (large graph, bottom right) of 3D-GRASE 1 (blue), 2D SE-EPI 1 (yellow), and 2D SE-EPI 3 (pink). Error bars represent standard errors across grid points (individual ROI graphs) and across regions (average graph). 

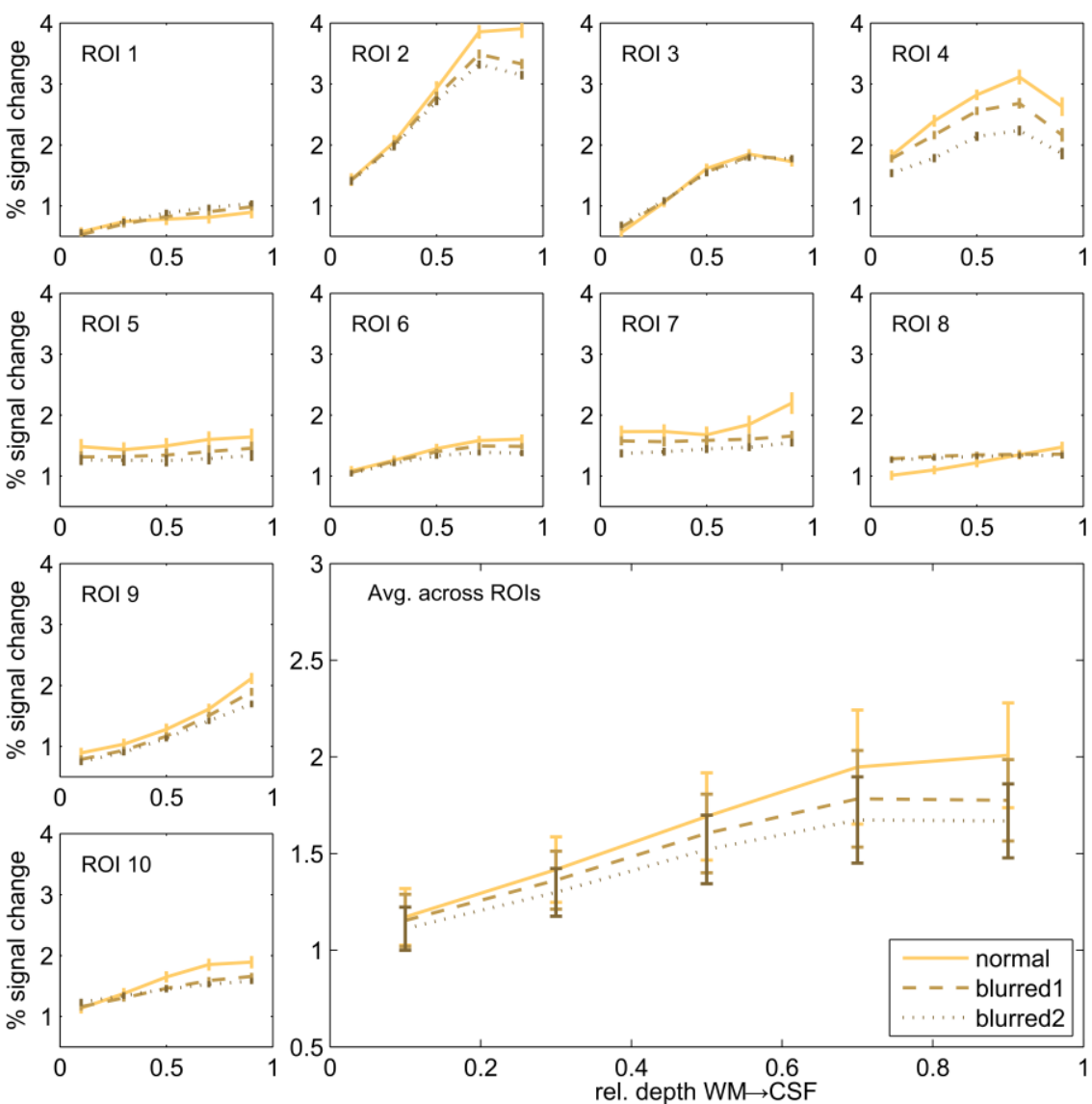

Figure 2-8: Cortical depth profiles in individual regions. Single regions (small graphs) and their average (large graph, bottom right) of 2D SE-EPI 1. Original data is compared to blurred data of two different blurring kernel sizes.

\section{Discussion}

In this study, we compared 3D-GRASE to 2D SE-EPI acquisitions in a high spatial resolution BOLD $\mathrm{FMRI}$ experiment at $7 \mathrm{~T}$. Simulations, PSF measurements and a simple visual activation paradigm with robust functional responses were used to assess differences with regard to voxel blurring, functional sensitivity, and functional (BOLD) specificity as indicated by cortical depth dependent profiles. Our findings suggest that the anisotropic blurring in 3D-GRASE is higher than in 2D SE-EPI. Further, 

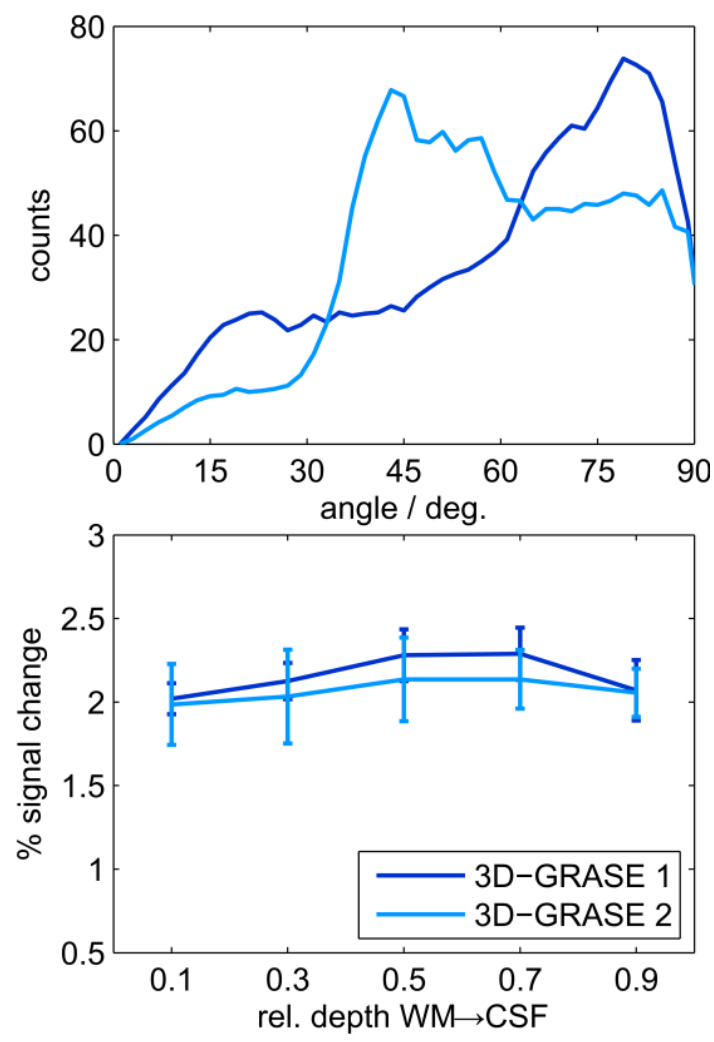

Figure 2-9: Comparison of 3D-GRASE 1 and 3D-GRASE 2. Top: Histograms of angle between cortex normal and partition encoding direction of both, 3DGRASE 1 (dark blue) and 3D-GRASE 2 (light blue), in degrees. Bottom: Mean cortical depth profiles. Error bars represent standard errors across regions.

3D-GRASE yields a more sensitive BOLD signal and less macrovascular weighting as indicated by the smaller superficial effect in the cortical depth profiles. Based on these findings, we conclude that with the used experimental set-up 3D-GRASE yields valuable advantages over 2D SE-EPI for high-resolution $\mathrm{FMRI}$ applications provided the more limited coverage is sufficient to the experimental setup. The ability to discriminate small, differential signals at a length-scale on the order of $1 \mathrm{~mm}$ depends on the product of tSNR and effect-size (Murphy et al., 2007), which is proportional to the contrast-to-noise ratio (CNR). The measured effect-size (i.e. difference in BOLD signal) depends on the image blurring, and hence depends on the orientation of the structure with respect to the anisotropic image blurring. It can be shown that, for the protocols employed here, the 
superior tSNR of 3D-GRASE outweighs detrimental effects of the stronger image blurring, and that the CNR will be below that of 2D SE-EPI only if the investigated structure is closely aligned ( $\sim$ deg.) with the partition direction of 3D-GRASE.

Our results are in general agreement with previous studies investigating 3D-GRASE (De Martino et al., 2013) or 2D SE-EPI (Goense and Logothetis, 2006a; Harel et al., 2006; Jin and Kim, 2008a), independently. The different protocols we used followed practical considerations and were performed similarly to previously published studies while also matching parameters such as resolution and echo time. Compared to previous studies, the imaging FoV of the 3D-GRASE acquisitions was reduced to account for the slower switching of the body gradient coil (SC72) that was used here.

Inner-volume selection was previously proposed to reduce the echo train length in 2D SE-EPI using spatially orthogonal refocusing pulses to acquire a single slice (Yacoub et al., 2007; Yacoub et al., 2008). Multi-slice acquisitions with this approach are sub-optimal because of saturation effects. Outer-volume suppression (OVS, Heidemann et al., 2012) and 2D spatially selective RF pulses (Pauly et al., 1989; Finsterbusch, 2010; Finsterbusch, 2013) have also been proposed. However, magnetization transfer effects in OVS would reduce the tSNR of 2D SE-EPI further, while additional RF-pulses would tighten the SAR constraints (Pfeuffer et al., 2002; Wargo and Gore, 2013) leading to a reduced number of slices. The use of segmented acquisitions would require techniques to mitigate the effects of subject motion (e.g. the use of bite-bars or advanced image reconstruction techniques) and would be significantly less time efficient. Finally, a surface receive coil with an even more limited FoV (Koning et al., 2013) was not available to us and may represent an alternative way to achieve zoomed imaging in 2D multi-slice imaging.

\section{Imaging point-spread function}

3D-GRASE was found to exhibit a wider PSF in partition direction than the 2D SE-EPI acquisitions in phase-encoding direction or slice direction (as expected from simulations). The blurring in the phase-encoding direction of 3D-GRASE was minimal because of the short EPI echo train duration due to the reduced FoV. 
The PSF was assessed based on non-phase-encoded data from the entire FoV. Two concomitant sources of possible confounds should be taken into consideration. First, different spatial locations have different PSF properties due to the relaxation properties of their underlying anatomical tissue. Since $\mathrm{T}_{2}$ of white matter and gray matter is almost identical at $7 \mathrm{~T}$ (Yacoub et al., 2003; Deistung et al., 2008; Cox and Gowland, 2010) and $T_{2}{ }^{*}$ of white matter is only slightly shorter (Peters et al., 2007; Deistung et al., 2008), one can expect this influence to be small. CSF, which has a much longer $T_{2}$, occupies only a small volume fraction in the chosen FoV and is partly saturated due to its long $\mathrm{T}_{1}\left(\mathrm{~T}_{1} \sim \mathrm{TR}\right)$. Other tissue compartments, which have much shorter $\mathrm{T}_{2}$, contribute relatively little to the signal at TE. It can therefore be expected that the presence of various tissue types does not significantly affect the PSF estimation. Second, static field inhomogeneities across the FoV lead to an overestimation of the global PSF by a broadening of the measured signal. Local off-resonances shift the voxel-by-voxel peak along the phase-encoding direction. The sum of the shifted peaks is broader than an individual one would be. Also, the effective volumetric $\mathrm{T}_{2}{ }^{*}$ is shortened. Either of these effects (tissue or field specific inhomogeneities) would lead to an overestimation or larger PSF. As such, our measurements can be regarded as a worst-case approximation of the local blurring. The simulations using gray matter $\mathrm{T}_{2}{ }^{*}$, on the other hand, reflect the ideal case and can be seen as a lower bound of the local blurring, not subject to either experimental imperfections affecting the local blurring or the global overestimation affecting only our measurements. The reasonable agreement of these numbers suggests that the detrimental effects of the global estimation are limited. An alternative approach, circumventing these possible confounds, would be to employ an acquisition scheme similar to the one by (Zeng and Constable, 2002; Chung et al., 2011), which was used for distortion correction in EPI images. In this study, we chose an approach that did not depend on image reconstruction, which is desirable when comparing different imaging pulse sequences.

This work focused on imaging related effects on the PSF and we did not consider the additional effect of the BOLD related spatial spread of the signal (i.e. the functional PSF), both of which need to be accounted for in determining the ultimate resolution of the fMRI signals. The latter is normally measured by considering differential paradigms with a known spatial distribution of responses and comparing it to the measured spatial 
distribution (Shmuel et al., 2007). In light of the results presented here, it is worth noting that a wider spatial imaging PSF will not cancel out high spatial frequency information in differential fMRI paradigms. Rather, when adjacent areas respond to different functional conditions, a wider spatial imaging PSF has a similar effect as a wider functional PSF and results in a reduction of CNR between the functional conditions (Yacoub et al., 2008). Thus, the combination of a differential fMRI paradigm, along with a high functional CNR with reduced contamination from pial veins, explain why columnar or cortical depth dependent fMRI analyses (Zimmermann et al., 2011; Olman et al., 2012; De Martino et al., 2013), with blurring levels (in 3D GRASE acquisitions) comparable to the data presented here, are possible.

\section{tSNR and BOLD sensitivity}

The sensitivity to detect small, significant BOLD changes was found to be clearly superior in 3D-GRASE in this study (Figures 2-5 and 2-6). This is a straight-forward consequence of the higher tSNR (Figure 2-4). The tSNR advantage of 3D-GRASE is likely to stem from two sources: First, being a 3D sequence 3D-GRASE is more SNR efficient because of the longer effective sampling time per volume (Edelstein et al., 1986). This is particularly relevant since such high-resolution $\mathrm{fMRI}$ typically operates in the thermally dominated noise regime (Triantafyllou et al., 2005), in which tSNR scales linearly with SNR. Second, the inner volume selection in 3D-GRASE obviated the need for parallel imaging in 3D-GRASE, whereas it was necessary in 2D SE-EPI causing a loss in static SNR and hence tSNR by under-sampling and g-factor penalty. Additionally, although the FWHM of the 2D SE-EPI acquisitions was wider in the slice direction than expected $(1.3 \pm 0.2$ times the nominal slice thickness) the large overlap of neighboring slices in the multi-slice $2 \mathrm{D}$ acquisitions reduces the available magnetization because of saturation effects (Figure 2-3). Improved slice profiles could therefore improve both $\mathrm{tSNR}$ and point-spread.

The origin of the greater sensitivity of the 3D-GRASE sequence seems not to originate from differences in functional contrast (i.e. 3D-GRASE did not exhibit greater response amplitudes, as seen in the functional response profiles, which is also consistent with less contribution from pial vessels), but rather the higher sensitivity seems to originate from a more robust signal (i.e. less noise). The higher sensitivity permitted 3D-GRASE to detect 
more subtle BOLD signal changes than 2D SE-EPI, while larger responses were detected about equally.

\section{Contrast mechanisms and functional specificity}

All acquisitions employed in this study elicit primarily $T_{2}$ weighted contrast, with varying contributions from other mechanisms. As such, the bulk of the detected BOLD signal changes have absolute amplitudes below five percent, in agreement with the literature (Yacoub et al., 2005), however, it should be noted that differences in tasks, acquisition parameters (e.g. echo time, Koopmans et al., 2011), or sampling strategies may all result in differences in the observed functional signal changes. Contributions from $\mathrm{T}_{2}{ }^{*}\left(\mathrm{~T}_{2}{ }^{\prime}\right)$ are not negligible due to the duration of the EPI echo trains, which was on the order of magnitude of the $\mathrm{T}_{2}{ }^{*}$ of the brain tissue. The $\mathrm{T}_{2}{ }^{*}$ contribution is expected to be higher in the 2D SE-EPI acquisitions than in 3D-GRASE because of the longer EPI echo trains and in-plane partial Fourier acquisitions, as discussed in the section on the image point-spread function. The later (non-center) partitions in the 3D-GRASE echo-train are sampled at a later effective TE than the nominal TE, which was matched with the TE of 2D SE-EPI 1. Therefore, the $T_{2}$ contribution is higher in 3DGRASE because later partitions experience more $T_{2}$ weighting and additional $T_{2}$ weighted stimulated echo weighting than the center partition (Goerke et al., 2007). In addition, blood vessels have a particularly sharp spatial profile (compared to smoother gray or white matter structures), which manifests itself in the k-space regions corresponding to high spatial frequencies. High k-space lines of an EPI readout, in turn, experience the strongest $\mathrm{T}_{2}{ }^{*}$ weighting. Note that, in reduced FoV acquisitions, the same effect takes place. However, given the shorter EPI readout times, the high k-space lines are acquired closer to the spin-echo, thus reducing the effect size.

Finally, more diffusion weighting is aggregated along the readout train of 3D-GRASE due to the readout and crusher gradients. Moderate diffusion weighting has been shown to suppress macrovascular signals (Lee et al., 1999; Duong et al., 2003; Yacoub et al., 2003).

In consequence of the aforementioned differences in contrast between 3DGRASE and 2D SE-EPI, the cortical depth profiles differ such that 2D SE-EPI exhibits an increase of the signal change towards the pial surface, whereas 3D-GRASE remains mostly flat. Such increasing cortical depth profiles have 
previously been associated with reduced functional specificity in SE-EPI (Goense and Logothetis, 2006a) and GE-EPI (Polimeni et al., 2010; De Martino et al., 2013), because they reflect $\mathrm{T}_{2}{ }^{*}$ induced weighting of macrovasculature. We follow this line of argument and conclude that under the experimental conditions employed here, 3D-GRASE is less biased by large superficial effects as indicated by the layer profiles (Figure 2-7).

Our imaging PSF results raise the question of whether the observed difference in layer profiles is a mere consequence of the stronger blurring in the partition direction of 3D-GRASE. We show that this is not the case in two ways. First, we smoothed the 2D SE-EPI 1 data with anisotropic Gaussian filters exceeding the width of the PSF of 3D-GRASE along the partition direction. The characteristics of cortical depth profiles, and more specifically the increase towards the pial surface, are preserved (Figure 28). Second, when looking at the angle between the partition encoding direction and the direction of the cortical depth (Figure 2-9 top), on average there is not a bias of alignment between the partition direction and the grid direction (i.e. smaller angles between the directions). The curvature of the cortex prevents this from occurring and ultimately protects the cortical depth measures from the partition blurring of both $3 \mathrm{D}$ GRASE 1 and 3D GRASE 2, despite their orthogonal prescription. Further, the differences in angle distributions between the two acquisitions did not even elicit any significant difference in the layer profiles (Figure 2-9).

\section{Acknowledgement}

This study was supported by European Research Council (ERC) grant 269853, National Institute of Neurological Disorders and Stroke (NINDS) P30 NS076408, Biomedical Technology Resource Centers (BTRC), National Center for Research Resources (NCRR) P41 RR08079, and the National Institute of Biomedical Imaging and Bioengineering (NIBIB) P41 EB015894. F.D.M. was funded by NWO VIDI (grant 864-13-012). 


\section{References}

Auerbach, E.J., Heberlein, K., and Hu, X. (2002). High-resolution T2 fMRI at high magnetic fields using PSIF. Proceedings of the 10th Annual Meeting of ISMRM, Honolulu, Hawaii, 2345.

Bandettini, P.A., Wong, E.C., Hinks, R.S., Tikofsky, R.S., and Hyde, J.S. (1992). Time course EPI of human brain function during task activation. Magn Reson Med 25, 390-397. doi: 10.1002/mrm.1910250220.

Bandettini, P.A., Wong, E.C., Jesmanowicz, A., Hinks, R.S., and Hyde, J.S. (1994). Spin-Echo and Gradient-Echo Epi of Human Brain Activation Using Bold Contrast - a Comparative-Study at $1.5 \mathrm{~T}$. Nmr in Biomedicine 7, 12-20. doi: 10.1002/nbm.1940070104.

Barth, M., Meyer, H., Kannengiesser, S.A., Polimeni, J.R., Wald, L.L., and Norris, D.G. (2010). T2-weighted 3D fMRI using S2-SSFP at 7 tesla. Magn Reson Med 63, 1015-1020. doi: 10.1002/mrm.22283.

Bernstein, M.A., King, K.F., and Zhou, X.J. (2004). Handbook of MRI pulse sequences. Elsevier.

Bieri, O., and Scheffler, K. (2007). Effect of diffusion in inhomogeneous magnetic fields on balanced steady-state free precession. NMR in Biomedicine 20, 1-10. doi: 10.1002/nbm.1079.

Boxerman, J.L., Hamberg, L.M., Rosen, B.R., and Weisskoff, R.M. (1995b). $\mathrm{Mr}$ Contrast Due to Intravascular Magnetic-Susceptibility Perturbations. Magnetic Resonance in Medicine 34, 555-566. doi: 10.1002/mrm.1910340412.

Boyacioglu, R., Schulz, J., Muller, N.C., Koopmans, P.J., Barth, M., and Norris, D.G. (2014). Whole brain, high resolution multiband spinecho EPI fMRI at 7T: A comparison with gradient-echo EPI using a color-word Stroop task. Neuroimage 97, 142-150. doi: 10.1016/j.neuroimage.2014.04.011.

Chung, J.Y., In, M.H., Oh, S.H., Zaitsev, M., Speck, O., and Cho, Z.H. (2011). An improved PSF mapping method for EPI distortion correction in human brain at ultra high field (7T). MAGMA 24, 179-190. doi: 10.1007/s10334-011-0251-1.

Constable, R.T., and Gore, J.C. (1992). The loss of small objects in variable TE imaging: implications for FSE, RARE, and EPI. Magn Reson Med 28, 9-24. doi: 10.1002/mrm.1910280103.

Constable, R.T., Kennan, R.P., Puce, A., Mccarthy, G., and Gore, J.C. (1994). Functional NMR imaging using fast spin echo at 1.5 T. Magn Reson Med 31, 686-690. doi: 10.1002/mrm.1910310617.

Cox, E.F., and Gowland, P.A. (2010). Simultaneous quantification of T2 and T'2 using a combined gradient echo-spin echo sequence at 
ultrahigh field. Magn Reson Med 64, 1440-1445. doi: 10.1002/mrm.22522.

De Martino, F., Zimmermann, J., Muckli, L., Ugurbil, K., Yacoub, E., and Goebel, R. (2013). Cortical depth dependent functional responses in humans at 7T: improved specificity with 3D GRASE. PLoS One 8, e60514. doi: 10.1371/journal.pone.0060514.

Deistung, A., Rauscher, A., Sedlacik, J., Stadler, J., Witoszynskyj, S., and Reichenbach, J.R. (2008). Susceptibility weighted imaging at ultra high magnetic field strengths: theoretical considerations and experimental results. Magnetic Resonance in Medicine 60, 11551168. doi: $10.1002 / \mathrm{mrm} .21754$.

Duong, T.Q., Yacoub, E., Adriany, G., Hu, X., Ugurbil, K., and Kim, S.G. (2003). Microvascular BOLD contribution at 4 and $7 \mathrm{~T}$ in the human brain: gradient-echo and spin-echo fMRI with suppression of blood effects. Magn Reson Med 49, 1019-1027. doi: 10.1002/mrm.10472.

Duong, T.Q., Yacoub, E., Adriany, G., Hu, X., Ugurbil, K., Vaughan, J.T., Merkle, H., and Kim, S.G. (2002). High-resolution, spin-echo BOLD, and CBF fMRI at 4 and 7 T. Magn Reson Med 48, 589-593.

Edelstein, W.A., Glover, G.H., Hardy, C.J., and Redington, R.W. (1986). The intrinsic signal-to-noise ratio in NMR imaging. Magn Reson Med 3, 604-618. doi: 10.1002/mrm.1910030413.

Feinberg, D.A., Hale, J.D., Watts, J.C., Kaufman, L., and Mark, A. (1986). Halving MR imaging time by conjugation: demonstration at $3.5 \mathrm{kG}$. Radiology 161, 527-531. doi: 10.1148/radiology.161.2.3763926.

Feinberg, D.A., Harel, N., Ramanna, S., Ugurbil, K., and Yacoub, E. (2008). Sub-millimeter Single-shot 3D GRASE with Inner Volume Selection for T2 weighted fMRI applications at 7 Tesla. 16th Annual Meeting International Society for Magnetic Resonance in Medicine, 2373.

Finsterbusch, J. (2010). Fast-spin-echo imaging of inner fields-of-view with 2D-selective RF excitations. J Magn Reson Imaging 31, 1530-1537. doi: 10.1002/jmri.22196.

Finsterbusch, J. (2013). Functional neuroimaging of inner fields-of-view with 2D-selective RF excitations. Magnetic Resonance Imaging 31, 1228-1235. doi: 10.1016/j.mri.2013.03.005.

Goa, P.E., Koopmans, P.J., Poser, B.A., Barth, M., and Norris, D.G. (2014). BOLD fMRI signal characteristics of S1- and S2-SSFP at 7 Tesla. Frontiers in Neuroscience 8. doi: 10.3389/fnins.2014.00049.

Goense, J., Merkle, H., and Logothetis, N.K. (2012). High-resolution fMRI reveals laminar differences in neurovascular coupling between positive and negative BOLD responses. Neuron 76, 629-639. doi: 10.1016/j.neuron.2012.09.019. 
Goense, J.B., and Logothetis, N.K. (2006a). Laminar specificity in monkey V1 using high-resolution SE-fMRI. Magn Reson Imaging 24, 381-392. doi: 10.1016/j.mri.2005.12.032.

Goerke, U., Van De Moortele, P.F., and Ugurbil, K. (2007). Enhanced relative BOLD signal changes in $\mathrm{T}(2)$-weighted stimulated echoes. Magn Reson Med 58, 754-762. doi: 10.1002/mrm.21369.

Gomori, J., Holland, G., Grossman, R., Gefter, W., and Lenkinski, R. (1988). Fat suppression by section-select gradient reversal on spin-echo MR imaging. Work in progress. Radiology 168, 493-495. doi: 10.1148/radiology.168.2.3393670.

Harel, N., Lin, J., Moeller, S., Ugurbil, K., and Yacoub, E. (2006). Combined imaging-histological study of cortical laminar specificity of fMRI signals. Neuroimage 29, 879-887. doi: 10.1016/j.neuroimage.2005.08.016.

Harmer, J., Sanchez-Panchuelo, R.M., Bowtell, R., and Francis, S.T. (2012). Spatial location and strength of BOLD activation in high-spatialresolution $\mathrm{fMRI}$ of the motor cortex: a comparison of spin echo and gradient echo fMRI at 7 T. NMR Biomed 25, 717-725. doi: 10.1002/nbm.1783.

Heidemann, R.M., Ivanov, D., Trampel, R., Fasano, F., Meyer, H., Pfeuffer, J., and Turner, R. (2012). Isotropic submillimeter $\mathrm{FMRI}$ in the human brain at $7 \mathrm{~T}$ : combining reduced field-of-view imaging and partially parallel acquisitions. Magn Reson Med 68, 1506-1516. doi: 10.1002/mrm.24156.

Hennig, J., Nauerth, A., and Friedburg, H. (1986). RARE imaging: a fast imaging method for clinical MR. Magn Reson Med 3, 823-833. doi: 10.1002/mrm.1910030602.

Huber, L., Goense, J., Kennerley, A.J., Trampel, R., Guidi, M., Reimer, E., Ivanov, D., Neef, N., Gauthier, C.J., Turner, R., and Moller, H.E. (2015a). Cortical lamina-dependent blood volume changes in human brain at 7 T. Neuroimage 107, 23-33. doi: 10.1016/j.neuroimage.2014.11.046.

Ivanov, D., Schafer, A., Streicher, M.N., Heidemann, R.M., Trampel, R., and Turner, R. (2010). A simple low-SAR technique for chemical-shift selection with high-field spin-echo imaging. Magn Reson Med 64, 319-326. doi: 10.1002/mrm.22518.

Jin, T., and Kim, S.-G. (2008a). Cortical layer-dependent dynamic blood oxygenation, cerebral blood flow and cerebral blood volume responses during visual stimulation. Neuroimage 43, 1-9. doi: 10.1016/j.neuroimage.2008.06.029.

Jones, S.E., Buchbinder, B.R., and Aharon, I. (2000). Three-dimensional mapping of cortical thickness using Laplace's equation. Human 
Brain Mapping 11, 12-32. doi: 10.1002/10970193(200009)11:1<12::aid-hbm20>3.0.co;2-k.

Koning, W., Bluemink, J.J., Langenhuizen, E.A., Raaijmakers, A.J., Andreychenko, A., Van Den Berg, C.A., Luijten, P.R., Zwanenburg, J.J., and Klomp, D.W. (2013). High-resolution MRI of the carotid arteries using a leaky waveguide transmitter and a high-density receive array at 7 T. Magn Reson Med 69, 1186-1193. doi: 10.1002/mrm.24345.

Koopmans, P.J., Barth, M., Orzada, S., and Norris, D.G. (2011). Multi-echo $\mathrm{fMRI}$ of the cortical laminae in humans at 7 T. Neuroimage 56, 1276-1285. doi: 10.1016/j.neuroimage.2011.02.042.

Kwong, K.K., Belliveau, J.W., Chesler, D.A., Goldberg, I.E., Weisskoff, R.M., Poncelet, B.P., Kennedy, D.N., Hoppel, B.E., Cohen, M.S., Turner, R., and Et Al. (1992). Dynamic magnetic resonance imaging of human brain activity during primary sensory stimulation. Proc Natl Acad Sci U S A 89, 5675-5679. doi: 10.1073/pnas.89.12.5675.

Lee, S.-P., Silva, A.C., Ugurbil, K., and Kim, S.-G. (1999). Diffusion-weighted spin-echo fMRI at $9.4 \mathrm{~T}$ : microvascular/tissue contribution to BOLD signal changes. Magnetic Resonance in Medicine 42, 919-928. doi: 10.1002/(SICI)1522-2594(199911)42:5<919::AID-MRM12>3.0.CO;28.

Mansfield, P. (1977). Multi-planar image formation using NMR spin echoes. Journal of Physics C: Solid State Physics 10, L55. doi: 10.1088/00223719/10/3/004.

Miller, K.L., and Jezzard, P. (2008). Modeling SSFP functional MRI contrast in the brain. Magn Reson Med 60, 661-673. doi: 10.1002/mrm.21690.

Mugler, J.P., 3rd, Epstein, F.H., and Brookeman, J.R. (1992). Shaping the signal response during the approach to steady state in threedimensional magnetization-prepared rapid gradient-echo imaging using variable flip angles. Magn Reson Med 28, 165-185. doi: 10.1002/mrm.1910280202.

Murphy, K., Bodurka, J., and Bandettini, P.A. (2007). How long to scan? The relationship between $\mathrm{FMRI}$ temporal signal to noise ratio and necessary scan duration. Neurolmage 34, 565-574. doi: 10.1016/j.neuroimage.2006.09.032.

Norris, D.G. (2012). Spin-echo fMRI: The poor relation? Neuroimage 62, 1109-1115. doi: 10.1016/j.neuroimage.2012.01.003.

Ogawa, S., Lee, T.M., Kay, A.R., and Tank, D.W. (1990). Brain magnetic resonance imaging with contrast dependent on blood oxygenation. Proc Natl Acad Sci U S A 87, 9868-9872. 
Ogawa, S., Tank, D.W., Menon, R., Ellermann, J.M., Kim, S.G., Merkle, H., and Ugurbil, K. (1992). Intrinsic signal changes accompanying sensory stimulation: functional brain mapping with magnetic resonance imaging. Proc Natl Acad Sci U S A 89, 5951-5955.

Olman, C.A., Harel, N., Feinberg, D.A., He, S., Zhang, P., Ugurbil, K., and Yacoub, E. (2012). Layer-specific fMRI reflects different neuronal computations at different depths in human V1. PLoS One 7, e32536. doi: 10.1371/journal.pone.0032536.

Oshio, K., and Feinberg, D.A. (1991). GRASE (Gradient- and spin-echo) imaging: a novel fast MRI technique. Magn Reson Med 20, 344349. doi: 10.1148/radiology.181.2.1924811.

Panchuelo, R.M.S., Schluppeck, D., Harmer, J., Bowtell, R., and Francis, S. (2015). Assessing the Spatial Precision of SE and GE-BOLD Contrast at 7 Tesla. Brain topography 28, 62-65.

Park, H.W., Kim, D.J., and Cho, Z.H. (1987). Gradient reversal technique and its applications to chemical-shift-related NMR imaging. Magn Reson Med 4, 526-536. doi: 10.1002/mrm.1910040604.

Park, J., Mugler, J.P., 3rd, Horger, W., and Kiefer, B. (2007). Optimized T1weighted contrast for single-slab 3D turbo spin-echo imaging with long echo trains: application to whole-brain imaging. Magn Reson Med 58, 982-992. doi: 10.1002/mrm.21386.

Pauly, J., Nishimura, D., and Macovski, A. (1989). A k-space analysis of small-tip-angle excitation. Journal of Magnetic Resonance (1969) 81, 43-56. doi: 10.1016/j.jmr.2011.09.023.

Peters, A.M., Brookes, M.J., Hoogenraad, F.G., Gowland, P.A., Francis, S.T., Morris, P.G., and Bowtell, R. (2007). T2* measurements in human brain at 1.5, 3 and 7 T. Magn Reson Imaging 25, 748-753. doi: 10.1016/j.mri.2007.02.014.

Pfeuffer, J., Van De Moortele, P.-F., Yacoub, E., Shmuel, A., Adriany, G., Andersen, P., Merkle, H., Garwood, M., Ugurbil, K., and Hu, X. (2002). Zoomed Functional Imaging in the Human Brain at 7 Tesla with Simultaneous High Spatial and High Temporal Resolution. Neurolmage 17, 272-286. doi: 10.1006/nimg.2002.1103.

Pflugfelder, D., Vahedipour, K., Uludag, K., Shah, N.J., and Stocker, T. (2011). On the numerically predicted spatial BOLD fMRI specificity for spin echo sequences. Magn Reson Imaging 29, 1195-1204. doi: 10.1016/j.mri.2011.07.015.

Polimeni, J.R., Fischl, B., Greve, D.N., and Wald, L.L. (2010). Laminar analysis of 7T BOLD using an imposed spatial activation pattern in human V1. Neuroimage 52, 1334-1346. doi: 10.1016/j.neuroimage.2010.05.005. 
Poser, B.A., and Norris, D.G. (2007). Fast spin echo sequences for BOLD functional MRI. MAGMA 20, 11-17. doi: 10.1007/s10334-006-0063$x$.

Robson, M.D., Gore, J.C., and Constable, R.T. (1997). Measurement of the point spread function in MRI using constant time imaging. Magn Reson Med 38, 733-740. doi: 10.1002/mrm.1910380509.

Shmuel, A., Yacoub, E., Chaimow, D., Logothetis, N.K., and Ugurbil, K. (2007). Spatio-temporal point-spread function of fMRI signal in human gray matter at 7 Tesla. Neuroimage 35, 539-552. doi: 10.1016/j.neuroimage.2006.12.030.

Triantafyllou, C., Hoge, R.D., Krueger, G., Wiggins, C.J., Potthast, A., Wiggins, G.C., and Wald, L.L. (2005). Comparison of physiological noise at $1.5 \mathrm{~T}, 3 \mathrm{~T}$ and $7 \mathrm{~T}$ and optimization of $\mathrm{fMRI}$ acquisition parameters. Neuroimage 26, 243-250. doi: 10.1016/j.neuroimage.2005.01.007.

Uludag, K., Muller-Bierl, B., and Ugurbil, K. (2009). An integrative model for neuronal activity-induced signal changes for gradient and spin echo functional imaging. Neuroimage 48, 150-165. doi: 10.1016/j.neuroimage.2009.05.051.

Van De Moortele, P.F., Auerbach, E.J., Olman, C., Yacoub, E., Ugurbil, K., and Moeller, S. (2009). T1 weighted brain images at 7 Tesla unbiased for Proton Density, T2* contrast and RF coil receive B1 sensitivity with simultaneous vessel visualization. Neuroimage 46, 432-446. doi: 10.1016/j.neuroimage.2009.02.009.

Volk, A., Tiffon, B., Mispelter, J., and Lhoste, J.-M. (1987). Chemical shiftspecific slice selection. A new method for chemical shift imaging at high magnetic field. Journal of Magnetic Resonance (1969) 71, 168174. doi: 10.1016/0022-2364(87)90141-7.

Wargo, C.J., and Gore, J.C. (2013). Localized high-resolution DTI of the human midbrain using single-shot EPI, parallel imaging, and outervolume suppression at 7 T. Magnetic Resonance Imaging 31, 810819. doi: 10.1016/j.mri.2013.01.013.

Yacoub, E., Duong, T.Q., Van De Moortele, P.F., Lindquist, M., Adriany, G., Kim, S.G., Ugurbil, K., and Hu, X. (2003). Spin-echo fMRI in humans using high spatial resolutions and high magnetic fields. Magn Reson Med 49, 655-664. doi: 10.1002/mrm.10433.

Yacoub, E., Harel, N., and Ugurbil, K. (2008). High-field fMRI unveils orientation columns in humans. Proc Natl Acad Sci U S A 105, 10607-10612. doi: 10.1073/pnas.0804110105.

Yacoub, E., Shmuel, A., Logothetis, N., and Ugurbil, K. (2007). Robust detection of ocular dominance columns in humans using Hahn Spin 
Echo BOLD functional MRI at 7 Tesla. Neuroimage 37, 1161-1177. doi: 10.1016/j.neuroimage.2007.05.020.

Yacoub, E., Van De Moortele, P.F., Shmuel, A., and Ugurbil, K. (2005). Signal and noise characteristics of Hahn SE and GE BOLD fMRI at $7 \mathrm{~T}$ in humans. Neuroimage 24, 738-750. doi: 10.1016/j.neuroimage.2004.09.002.

Zaitsev, M., Hennig, J., and Speck, O. (2004). Point spread function mapping with parallel imaging techniques and high acceleration factors: fast, robust, and flexible method for echo-planar imaging distortion correction. Magn Reson Med 52, 1156-1166. doi: 10.1002/mrm.20261.

Zeng, H., and Constable, R.T. (2002). Image distortion correction in EPI: comparison of field mapping with point spread function mapping. Magn Reson Med 48, 137-146. doi: 10.1002/mrm.10200.

Zimmermann, J., Goebel, R., De Martino, F., Van De Moortele, P.F., Feinberg, D., Adriany, G., Chaimow, D., Shmuel, A., Ugurbil, K., and Yacoub, E. (2011). Mapping the organization of axis of motion selective features in human area MT using high-field fMRI. PLoS One 6, e28716. doi: 10.1371/journal.pone.0028716. 
Chapter 2 


\section{Chapter 3}

\section{Variable flip angle 3D-GRASE for high resolution $\mathrm{fMRI}$ at $7 \mathrm{~T}$}

Corresponding publication: Kemper, Valentin G.; De Martino, Federico; Yacoub, Essa; Goebel, Rainer (2015). "Variable flip angle 3D-GRASE for high resolution fMRI at 7 T.", Magn. Res. Med. 


\section{Abstract}

This study evaluates the use of variable flip angle refocusing pulse trains in single-shot 3D gradient and spin-echo (3D-GRASE) in order to reduce blurring and increase the spatial coverage for high spatial resolution $\mathrm{T}_{2}$ weighted functional MRI at $7 \mathrm{~T}$. Variable flip angle refocusing schemes in 3D-GRASE were calculated based on extended phase graph theory. The blurring along the slice (partition) direction was evaluated in simulations, as well as phantom and in-vivo experiments. Further, temporal stability and functional sensitivity at $0.8 \mathrm{~mm}$ isotropic resolution were assessed. Variable flip angle refocusing schemes yielded significantly reduced blurring compared to conventional refocusing schemes, with the full width at half maximum being about $30-40 \%$ narrower. Simultaneously, spatial coverage could be increased by $80 \%$. The temporal signal-to-noise ratio was slightly reduced, but functional sensitivity was largely maintained due to increased functional contrast in the variable flip angle acquisitions. Signal-to-noise ratio and functional sensitivity were reduced more strongly in areas with insufficient RF transmission indicating higher sensitivity to experimental imperfections. We conclude that variable flip angle refocusing schemes increase usability of 3D-GRASE for high-resolution fMRI by reducing blurring and allowing increased spatial coverage. 


\section{Introduction}

Simulations and experiments have shown that at ultra-high field (i.e. $7 \mathrm{~T}$ and above) the $T_{2}$ weighted blood oxygen level dependent (BOLD) signal is more specific to the site of neuronal activity than $\mathrm{T}_{2}{ }^{*}$ weighted BOLD (at either high or low field), because $T_{2}$ weighted BOLD is less sensitive to large draining veins (Duong et al., 2003; Yacoub et al., 2005; Uludag et al., 2009). However, regardless of the increased signal to noise ratio available at higher field (Edelstein et al., 1986; Pohmann et al., 2015), purely $\mathrm{T}_{2}$ weighted acquisition strategies (Constable et al., 1994; Poser and Norris, 2007; Barth et al., 2010; Goerke et al., 2011; Hua et al., 2013b; Goa et al., 2014) have been hindered in high-resolution applications by their long acquisition time, lower sensitivity, and/or the high specific absorption rate (SAR). The temporal efficiency and (relative) SAR parsimony of spin-echo echo planar imaging (SE-EPI, Bandettini et al., 1994) partly alleviate these issues. However, EPI readouts introduce $\mathrm{T}_{2}{ }^{*}$ contrast, depending on the echo train length, compromising purely $\mathrm{T}_{2}$ weighted contrast (Goense and Logothetis, 2006b). To counteract this, outer-volume suppression (Pfeuffer et al., 2002; Heidemann et al., 2012), inner-volume selection by orthogonal radio-frequency (RF) pulse plain prescriptions (Feinberg et al., 1985), and segmented EPI trains have been used (Yacoub et al., 2005).

In the study of human brain function, the successful use of the latter two methods has been showcased by a single-slice 2D SE-EPI with anisotropic resolution (Yacoub et al., 2007; Yacoub et al., 2008). Despite this success, the method did not find applicability outside a flat piece of cortex in primary visual areas. Extending this approach, to overcome the limitation of acquiring a single anisotropic slice, 3D inner-volume gradient and spin echo (3D-GRASE, Feinberg and Oshio, 1991; Feinberg et al., 2008) has been proposed. 3D-GRASE acquires a full 3D volume after a single excitation pulse by sampling k-space with a combination of EPI echo trains and multiple spin-echoes. The refocusing RF pulses select a perpendicular slab achieving inner-volume selection. We have recently shown that 3D-GRASE is functionally more specific and more sensitive than 2D SE-EPI with a large field of view (FoV) (Kemper et al., 2015b), however, blurring along the secondary phase-encoding direction (partition direction) and the small FoV of 3D-GRASE still limit its usability. In particular, the FoV of 3D-GRASE cannot easily be extended as doing so introduces $T_{2}$ decay induced blurring (additionally $\mathrm{T}_{2}{ }^{*}$ blurring and $\mathrm{T}_{2}{ }^{*}$ functional contrast when increasing the in- 
plane FoV). To alleviate the blurring introduced by $T_{2}$ decay, the signal strength would have to be sustained throughout the echo train (Qin, 2012). This can be achieved by variable flip angle refocusing RF pulse trains, which introduce alternative signal pathways (stimulated echoes and additional primary spin-echoes of various pairs of RF pulses). They are designed based on extended phase graph theory (Hennig, 1988) and are routinely employed in $\mathrm{T}_{2}$ weighted turbo spin echo (RARE/FSE/TSE) (Hennig et al., 1986) structural imaging (known as SPACE, VISTA or CUBE) (Busse et al., 2006; Busse et al., 2008).

In this proof-of-concept study, we apply VFA refocusing trains in high spatial resolution single-shot 3D-GRASE. We validate the method in phantom acquisitions and demonstrate two cases of successful in-vivo application to (a) reduce the blurring and to (b) simultaneously reduce blurring and extend the imaging FoV. Accelerated imaging (Pruessmann et al., 1999; Griswold et al., 2002) as a potential alternative to increase the inplane FoV is also explored.

\section{Methods}

All measurements were performed on a 7T Magnetom Siemens scanner equipped with a body gradient system $(70 \mathrm{mT} / \mathrm{m}$ maximum amplitude, $200 \mathrm{mT} / \mathrm{m} / \mathrm{s}$ maximum slew rate). A 32-channel phased-array head coil (Nova Medical, Wilmington, MA, USA) was used in phantom measurements and a 16-channel phased array surface coil (Live Services, Minneapolis, MN, USA) covering the posterior part of the subjects' heads was used for in-vivo experiments. Eight healthy volunteers ( 5 female; mean age $27 \pm 4$ years) were scanned after giving informed written consent and in compliance with the local ethical board. One subject ( $(5)$ was excluded from functional analyses, because she reported sleepiness after the measurements. Second order automatic static field shim routines were applied and refined manually to reach good static field homogeneity (in-vivo: Larmor frequency full width at half maximum $\leq 45 \mathrm{~Hz}, \mathrm{~T}_{2}{ }^{*}=15.1 \pm 1.4 \mathrm{~ms}$ ). RF transmission voltage was adjusted based on a pre-saturation based $B_{1}$ mapping sequence provided by the scanner vendor. $A$ small region of interest in the center of the imaging volume was used to determine the correct voltage. 


\section{Variable flip angle refocusing design}

The variable flip angle pulse trains were calculated offline using MATLAB (The MATHWORKS Inc., Natick, MA, USA). It was assumed that the signal evolution along the partition encoding direction could be treated separately from the decay due to $T_{2}$ and $T_{2}{ }^{\prime}$, which affects the phase encoding direction. Further, it was assumed that the Carr-PurcellMeiboom-Gill (CPMG) condition was met for the central spin-echoes of the EPI echo trains. An exponential target signal decay was calculated taking into account TE, number of slices (also accounting for k-space truncation), partial Fourier (assuming zero-filling), the desired maximum blurring along the partition-direction, and the relaxation properties of the target tissue ( $\mathrm{T}_{2}=40 \mathrm{~ms}, \mathrm{~T}_{1}=400 \mathrm{~ms}$ for agar phantom, $\mathrm{T}_{2}=50 \mathrm{~ms}, \mathrm{~T}_{1}=1800 \mathrm{~ms}$ for gray matter). The calculation of the VFA echo train for a given target signal shape was then done as suggested in (Busse et al., 2006) based on (Hennig, 1988; Hennig et al., 2003). Finally, the flip angles of the last third of the echo train were raised quadratically in the in-vivo scans to maximize the signal intensity. The last refocusing flip angle was raised to the maximum of the echo train rather than to $180^{\circ}$ that would otherwise require longer refocusing pulses. Care was taken that the resulting signal intensities of the alternated last refocusing flip angles were all higher than in the exponential decay to ensure blurring would not become stronger due to the alternation.

\section{Point-spread function measurements}

Global point-spread function measurements were performed by acquiring non-Fourier-encoded images (Kemper et al., 2015b). After four volume acquisitions in order to reach a steady state, the phase-encoding gradients in the in-plane and partition directions were switched off for the fifth repetition. Instead of sampling a full k-space, the k-space center line was acquired repeatedly in every read-out, modulated by the modulation transfer function (MTF). The resultant data can be reconstructed in the reconstruction pipeline exhibiting the anisotropic image point-spread function (PSF), reflecting effects of the acquisition (i.e. signal modulation due to $\mathrm{T}_{2}$ and $\mathrm{T}_{2}{ }^{*}$ decay) and the reconstruction (i.e. filtering) (Mugler et al., 1992; Zaitsev et al., 2004; Park et al., 2007). The ideal case of a homogeneous MTF would result in a sharply peaked intensity distribution along the non-encoded directions. However, in practice, the obtained PSF 
has a finite width. In order to characterize the PSF, we computed the full width at half maximum (FWHM) by interpolating the magnitude of the PSF using a spline interpolation. This approach was chosen rather than a Gaussian fit (Kemper et al., 2015b) because it characterized the different peak shapes of the VFA acquisitions better.

\section{Phantom measurements}

A spherical agar phantom was used to test the VFA approach systematically in a wide range of parameter combinations. VFA pulse trains were calculated for each of the $4 \times 3 \times 3$ combinations of 1 ) FWHM of PSFs of 2 , $2.5,3$, and 4 pixels, 2) coverage of 10, 16, and 20 slices, and 3) partial Fourier acquisition of $5 / 8,7 / 8$, and $8 / 8$ (except Partial Fourier $5 / 8$ at 2 pixel FWHM was not performed with 10 slices because it would require a constant MTF). Other imaging parameters were as in the in-vivo REF measurement (see below).

\section{$\underline{\text { In-vivo measurements }}$}

Four different 3D-GRASE measurements were acquired with each subject: 1) a conventional 3D-GRASE acquisition with nominal $180^{\circ}$ refocusing pulses (REF); 2) a variable flip angle acquisition with otherwise same protocol as REF with reduced blurring (VFA1); 3) a variable flip angle acquisition to extend the FoV in partition-direction and with reduced blurring (VFA2); 4) the same protocol as REF, except GRAPPA factor 2 and doubled FoV in in-plane phase encoding direction (R2). TE in VFA1 and VFA2 could be slightly reduced compared to REF because of the shorter RF pulse lengths required for the smaller flip angles. See Table 3-1 for additional imaging parameters. The nominal refocusing flip angles used for VFA1 were $\left(109^{\circ}, 63^{\circ}, 62^{\circ}, 63^{\circ}, 75^{\circ}, 109^{\circ}\right)$, and for VFA2 $\left(90^{\circ}, 49^{\circ}, 45^{\circ}, 43^{\circ}\right.$, $43^{\circ}, 43^{\circ}, 42^{\circ}, 43^{\circ}, 46^{\circ}, 51^{\circ}, 58^{\circ}, 67^{\circ}, 77^{\circ}, 90^{\circ}$ ). All acquisitions used $90^{\circ}$ nominal excitation flip angles, linear phase ordering in in-plane direction, and centric ordering of the partition direction. 


\begin{tabular}{rcccc}
\hline & REF & VFA1 & VFA2 & R2 \\
\hline TE / TR (ms) & $37.9 /$ & $36.9 /$ & $35.9 /$ & $37.9 /$ \\
& 2000 & 2000 & 2000 & 2000 \\
Matrix size & $120 \times 30 \times 1$ & $120 \times 30 \times 1$ & $120 \times 30 \times 1$ & $120 \times 60 \times 1$ \\
& 0 & 0 & 8 & 0 \\
Image volume $\left(\mathrm{mm}^{3}\right.$ ) & $96 \times 24 \times 8$ & $96 \times 24 \times 8$ & $96 \times 24 \times 14$. & $96 \times 48 \times 8$ \\
& & & 4 & \\
Turbo factor & 6 & 6 & 14 & 6 \\
Echo train duration & 227 & 221 & 504 & 227 \\
(ms) & & & & \\
Nominal refocusing flip & $180^{\circ}$ & $62^{\circ}$ to & $42^{\circ}$ to $90^{\circ}$ & $180^{\circ}$ \\
angles & & $109^{\circ}$ & & \\
Echo-spacing (ms) & 1.03 & 1.03 & 1.03 & 1.03 \\
\hline
\end{tabular}

Table 3-1: Imaging parameters used in in-vivo measurements.

A $T_{1}$ weighted MPRAGE sequence (isotropic resolution of $0.6 \mathrm{~mm}$; $\mathrm{TR}=3100 \mathrm{~ms} ; \quad \mathrm{TI}=1500 \mathrm{~ms} ; \quad \mathrm{TE}=2.52 \mathrm{~ms} ; \quad$ Flip angle $=5^{\circ} ; \quad$ GRAPPA acceleration factor $=3$; FoV $=230 \times 230 \times 154 \mathrm{~mm}^{3}$; duration $8: 49 \mathrm{~min}$.) was used to acquire structural images for anatomical reference. Additionally, a proton density weighted MPRAGE with identical imaging parameters except without an inversion module (and: TR=1440 ms, duration 4:06 min.) was acquired to alleviate inhomogeneities across the FoV in postprocessing by dividing the $\mathrm{T}_{1}$ weighted image by the proton density weighted images (Van de Moortele et al., 2009).

Visual stimulation paradigm and functional data analysis

Subjects fixated a central point on a screen in the magnet bore while high contrast concentric flickering checkerboard patterns were displayed (visual angle of 39 degrees) via an angulated mirror attached to the RF coil. One run consisted of 36 blocks of 8 seconds stimulation interspersed with 10 to 14 seconds rest totaling a duration of 12:12 minutes.

Data analysis was performed using BrainVoyager QX 2.8.2 (Brain Innovation, Maastricht, The Netherlands) and custom-written routines in MATLAB. Preprocessing of the FMRI data included 3D rigid body motion correction and temporal high-pass filtering by a GLM with four cosine cycles and linear trend. The data were then co-registered with the structural images using positional information of the acquisitions. Manual 
adjustments were performed using edge information of the fMRI data. Finally, functional images were resampled in the 3D volume at a resolution of $0.8 \mathrm{~mm}$ using sinc interpolation.

A standard GLM was used to assess the functional activation. The visual stimulation regressor was created by convolving the visual activation blocks with a standard 2-gamma-function hemodynamic response function. Each run was analyzed on a single subject level with confound motion regressors ( 3 translational and 3 rotational).

To produce temporal signal-to-noise ratio (tSNR) maps, preprocessing included only high-pass filtering. No motion correction was applied to avoid additional spatial interpolation. The effect of the visual stimulation was removed from the voxels' time course using a GLM. We then computed tSNR dividing the voxels' temporal mean by the temporal standard deviation.

\section{Results}

\section{Phantom measurements}

Figure 3-1 displays the employed RF pulse trains used for the phantom measurements and the corresponding measured and simulated echo signal intensities. Measured data were coil-combined using sum-of-squares. As expected, smaller flip angles are employed in order to sustain the signal longer throughout the echo train. Good agreement between theoretical prediction and experimental results can be observed for all parameter combinations.

$\rightarrow$ Figure 3-1: (a) RF pulse flip angles employed in the phantom measurements for different numbers of slices (rows), different partial Fourier factors (columns) and different target PSF FWMH (in pixels, graph colors, annotated in top right graph). (b) Corresponding signal intensities. Markers and lines represent experimental data and simulations, respectively. Simulations and experimental data were scaled by their individual means. Size: Double-column width. 

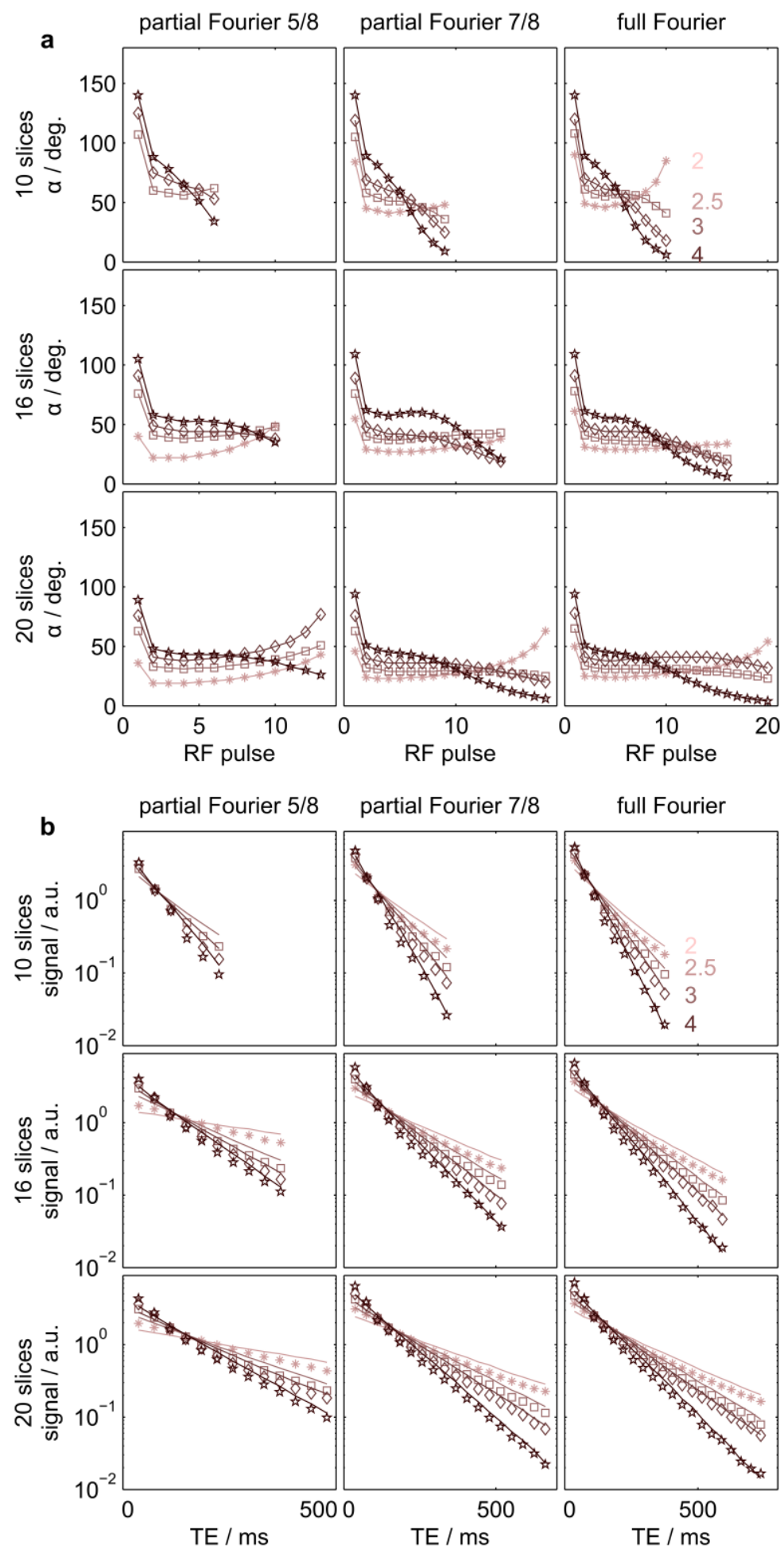
$\underline{\text { Point-spread function simulations and measurements }}$

The results of the simulations and measurements of in-vivo PSF in partition direction are summarized in Table 3-2. As expected, VFA1 and VFA2 have narrower PSFs than the REF acquisition in both measurements and simulations.

\begin{tabular}{lll}
\hline Acquisition & Measurement & Simulation \\
\hline REF & $4.0 \pm 0.5$ & 4.8 \\
VFA1 & $2.6 \pm 0.3$ & 2.3 \\
VFA2 & $2.9 \pm 0.3$ & 2.3 \\
\hline
\end{tabular}

Table 3-2: FWHM (pixel, mean \pm std) of PSF in in-vivo measurements.

\section{Temporal signal-to-noise ratio}

Mean signal and tSNR maps of a representative subject are shown in Figure 3-2 (all subjects in Figure 3-3). The reference acquisition, REF, yields the strongest tSNR; VFA1 and VFA2 have slightly reduced tSNRs. The GRAPPA acquisition, R2, has poor tSNR in comparison to the other acquisitions. All maps reveal reduced tSNR values in the left hemisphere (right side of the image, radiological convention) associated with RF transmission asymmetry. This reduction is more pronounced in the VFA acquisitions. 

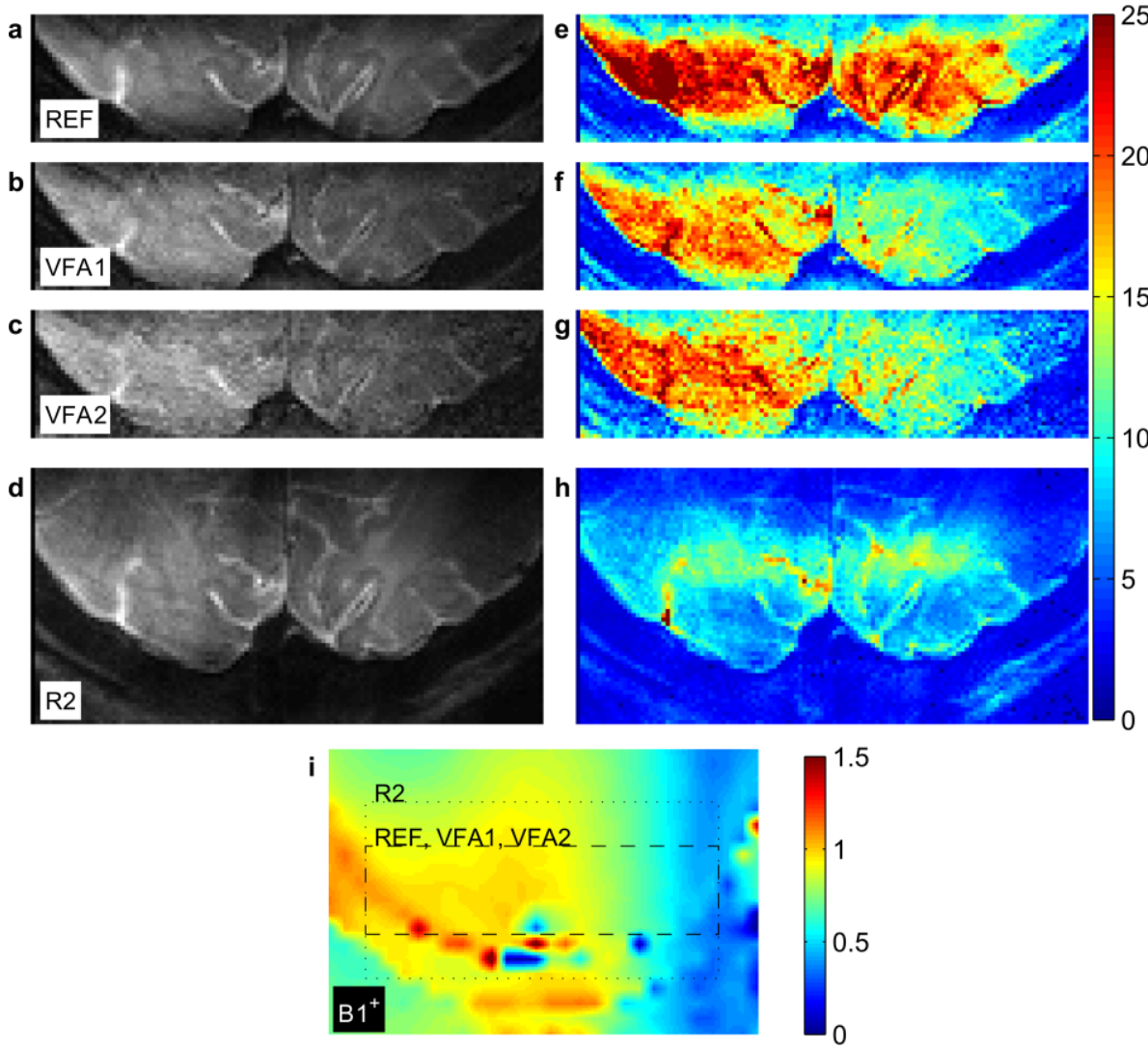

Figure 3-2: (a-d) Mean signal maps of REF (top), VFA1 (top middle), VFA2 (bottom middle), and R2 (bottom). A single, transversal oblique central slice is shown for each acquisition (identical anatomical position). (e-h) Temporal signal to noise ratio maps at position corresponding to maps in ad. The same color scale is used for all maps. (i) Relative RF transmission $\left(\mathrm{B}_{1}{ }^{+}\right)$map of same location and interpolated to same resolution. The inplane imaging FoVs of the functional acquisitions are indicated by the dashed (REF, VFA1, VFA2) and dotted (R2) boxes, respectively. 

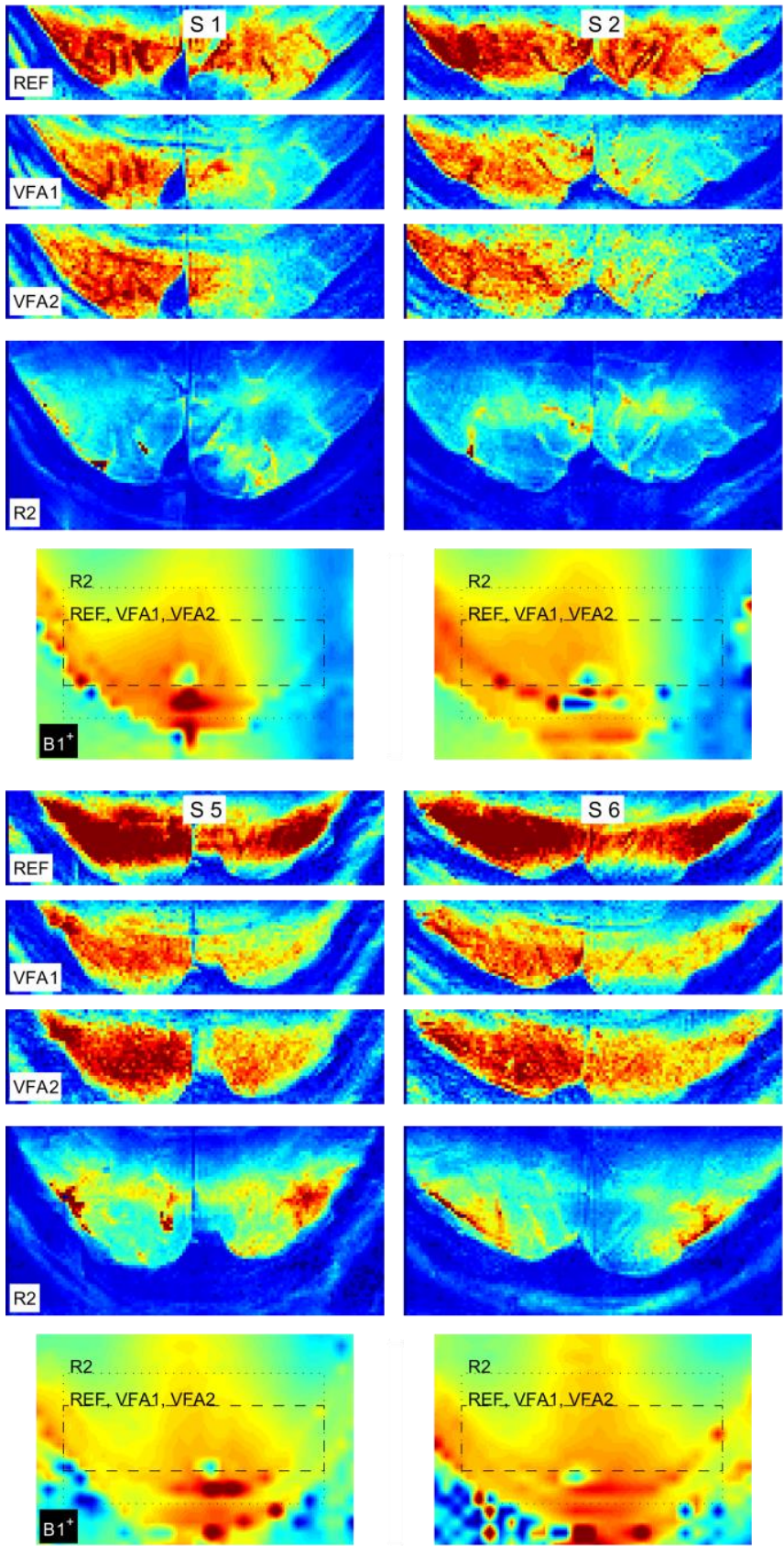

Figure 3-3 (double page): Temporal signal to noise ratio maps of all subjects (S $1-\mathrm{S} 8$ ) and all acquisitions. Single, transversal oblique central slices (identical anatomical position, same color scale) are shown for each acquisition. 

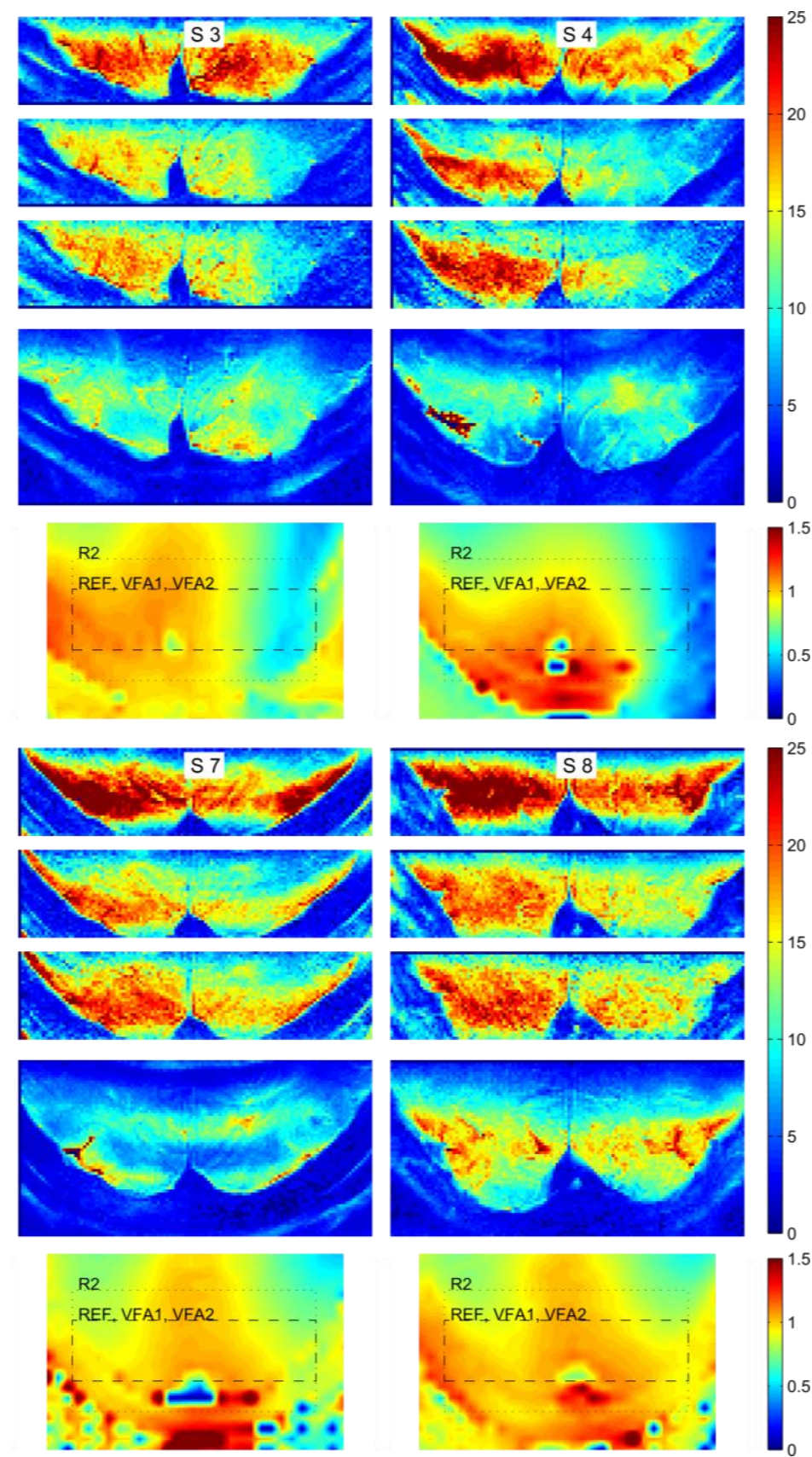

Bottom: Relative RF transmission $\left(\mathrm{B}_{1}^{+}\right)$maps of same location and interpolated to same resolution. The in-plane imaging FoVs of the functional acquisitions are indicated by the dashed (REF, VFA1, VFA2) and dotted (R2) boxes, respectively. 


\section{Functional activation}

Maps of functional activation of a representative subject are displayed in Figure 3-4. All displayed acquisitions, REF (top), VFA1 (middle), and VFA2 (bottom), show robust activation patterns. The strongest activation is confined to regions of gray matter and (in smaller proportion) CSF. The GRAPPA acquisition, R2, is not shown because it revealed very little functional responses. Figure 3-5 displays activation maps of all subjects.

To quantify the differences, we calculated within-subject medians of absolute percent signal changes and absolute t-values for all acquisitions using a gray matter mask derived from the anatomical reference data in the mutually covered volume. BOLD signal changes were found to be $14 \%$ (18\%) higher in VFA1 (VFA2) than in REF $(p=0.02$, uncorr., two-sided Wilcoxon sign rank test). Further, VFA1 and VFA2 did not reveal significantly different $t$-values than REF. R2 had significantly lower t-values than the other acquisitions $(p<0.02)$, although BOLD signal changes were not significantly different from REF, indicating the same contrast mechanism as in REF but lower tSNR. Individual subject data is provided in Table 3-3 and Figure 3-6.

$\rightarrow$ Figure 3-4: Visual activation patterns (t-values, two-tailed Student's ttest, $p<0.01$, uncorrected, 4-voxel cluster-threshold) overlaid on anatomical reference in sagittal, transversal, and coronal cuts. The imaging volume cross section is indicated by the colored boxes in the sagittal cuts and the anatomical reference image at the top (blue: REF, light green: VFA1, dark green: VFA2). Size: Double-column width. 
Variable flip angle 3D-GRASE
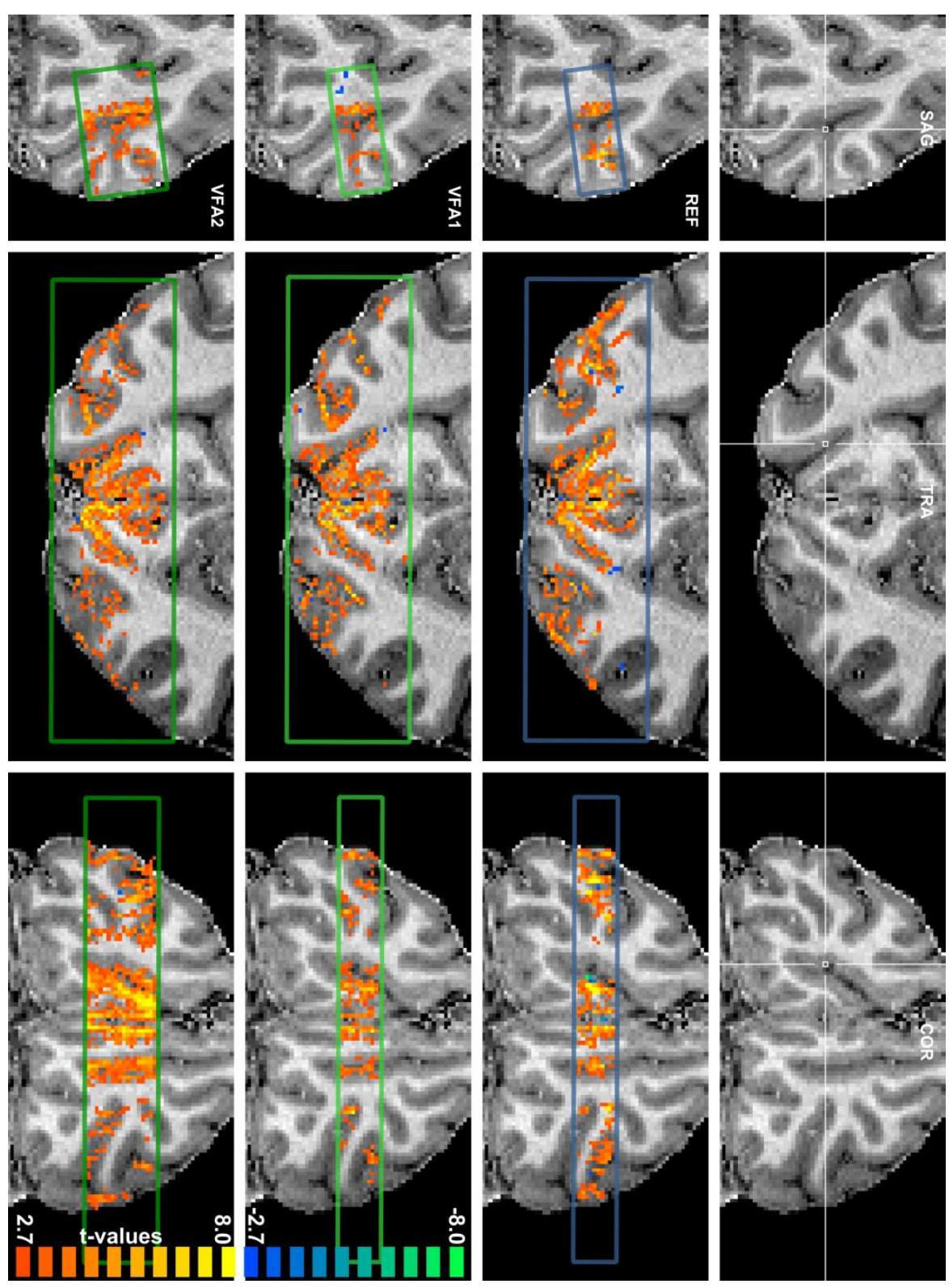


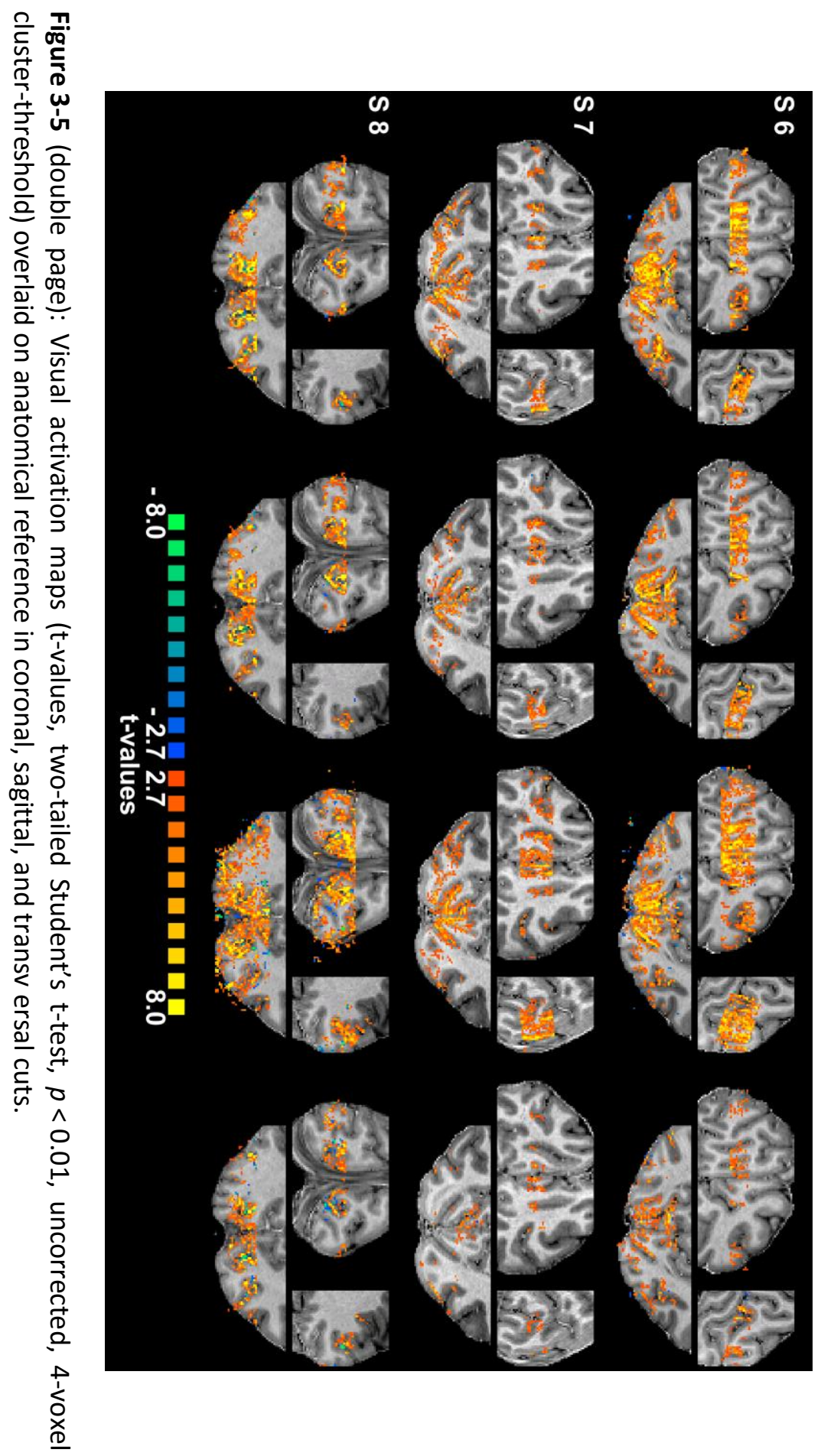




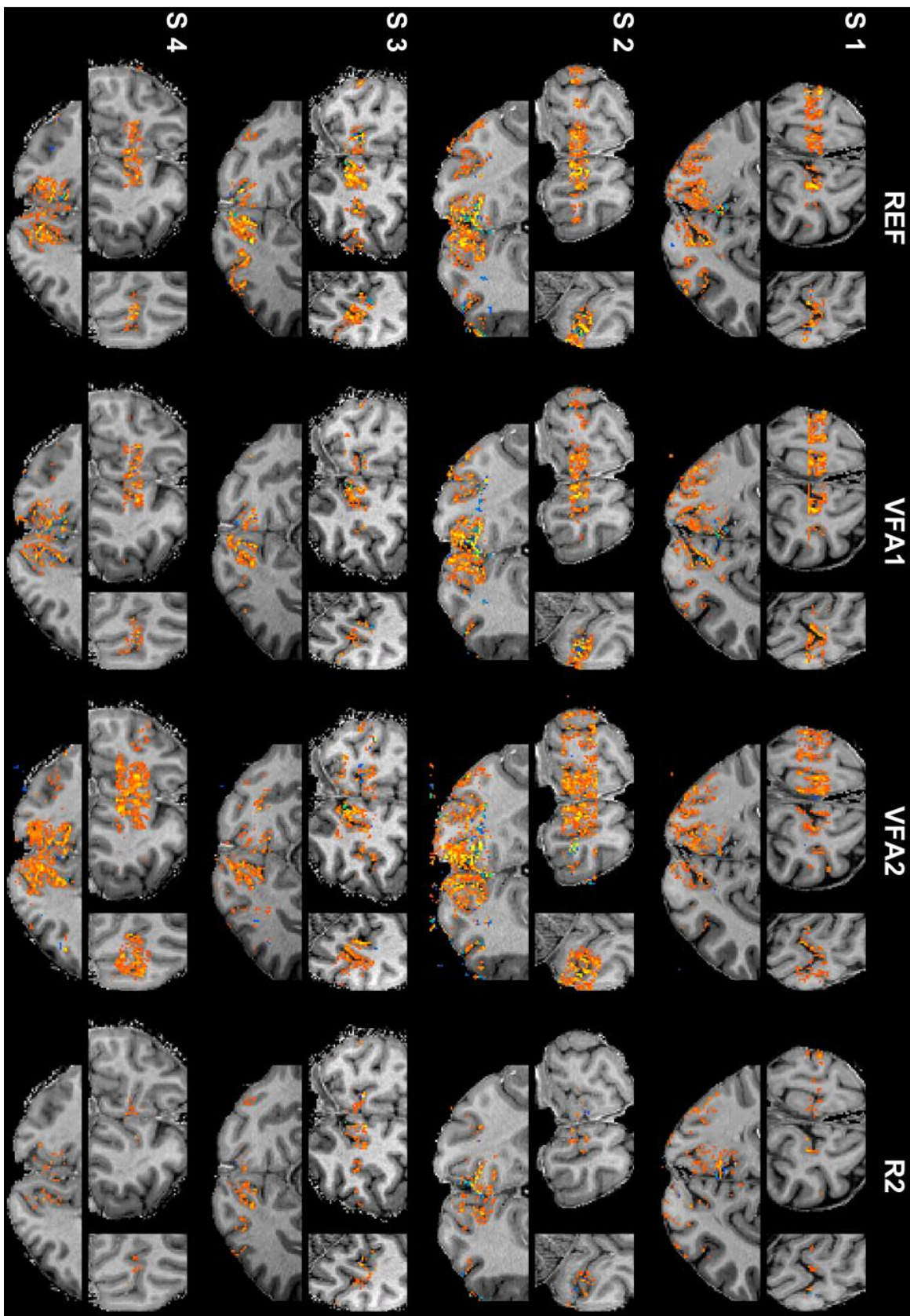




\section{Discussion and Conclusions}

We demonstrate the application of variable flip angle refocusing schemes in high resolution 3D-GRASE acquisitions in order to improve the image PSF and spatial coverage in $T_{2}$ weighted submillimeter $\mathrm{fMRI}$ applications at $7 \mathrm{~T}$. In short, the variable flip angle refocusing approach reduces the signal intensity at early echo times and increases it at later echo times. This results in the following: a) Reduced blurring across partitions and/or increased coverage. b) reduced tSNR (in center-out reordering). c) Increased BOLD contrast. We showed that the enhanced BOLD contrast largely counterbalances the reduced tSNR in VFA while image blurring was reduced and therefore conclude that VFA refocusing is a valuable improvement of the single-shot inner-volume 3D-GRASE sequence.

Our method is similar to that of Liang et al. (2014), who recently presented a VFA 3D-GRASE technique for low-resolution whole-brain perfusion measurements using arterial spin labeling at lower field to improve the PSF. Although the scope is different, the employed VFA refocusing RF pulse trains are similar because the ratio of sequence timings and tissue $T_{2}$ at $3 T$ and $7 \mathrm{~T}$ are comparable. However, it should be noted that the stronger $\mathrm{T}_{2}$ weighted stimulated-echo contrast induced by the VFA refocusing has different implications in BOLD imaging than in perfusion imaging, where minimal $\mathrm{T}_{2}$ contamination is desirable. Further, our study extends the quantitative assessment of the blurring by direct, empirical in-vivo measurements of the PSF.

\begin{tabular}{lcccccccc}
\hline & \multicolumn{3}{c}{ t-values } & \multicolumn{4}{c}{ Percent signal changes } \\
\cline { 5 - 8 } Subject & REF & VFA1 & VFA2 & R2 & REF & VFA1 & VFA2 & R2 \\
& & & & & & & & \\
\hline 1 & 1.61 & 1.65 & 1.43 & 1.15 & 1.20 & 1.46 & 1.47 & 1.29 \\
2 & 1.85 & 1.69 & 1.95 & 1.07 & 1.26 & 1.40 & 1.58 & 1.37 \\
3 & 1.48 & 1.15 & 1.27 & 1.06 & 1.10 & 1.11 & 1.19 & 1.06 \\
4 & 1.15 & 1.18 & 1.48 & 0.88 & 0.80 & 1.08 & 0.81 & 1.00 \\
6 & 2.12 & 1.95 & 2.10 & 1.44 & 1.22 & 1.40 & 1.44 & 1.31 \\
7 & 1.62 & 1.50 & 1.70 & 1.11 & 1.06 & 1.19 & 1.25 & 1.24 \\
8 & 2.26 & 2.36 & 2.57 & 1.83 & 1.33 & 1.59 & 1.74 & 1.28 \\
\hline
\end{tabular}

Table 3-3: Individual subject medians of $t$-values and percent signal changes of all acquisitions. 


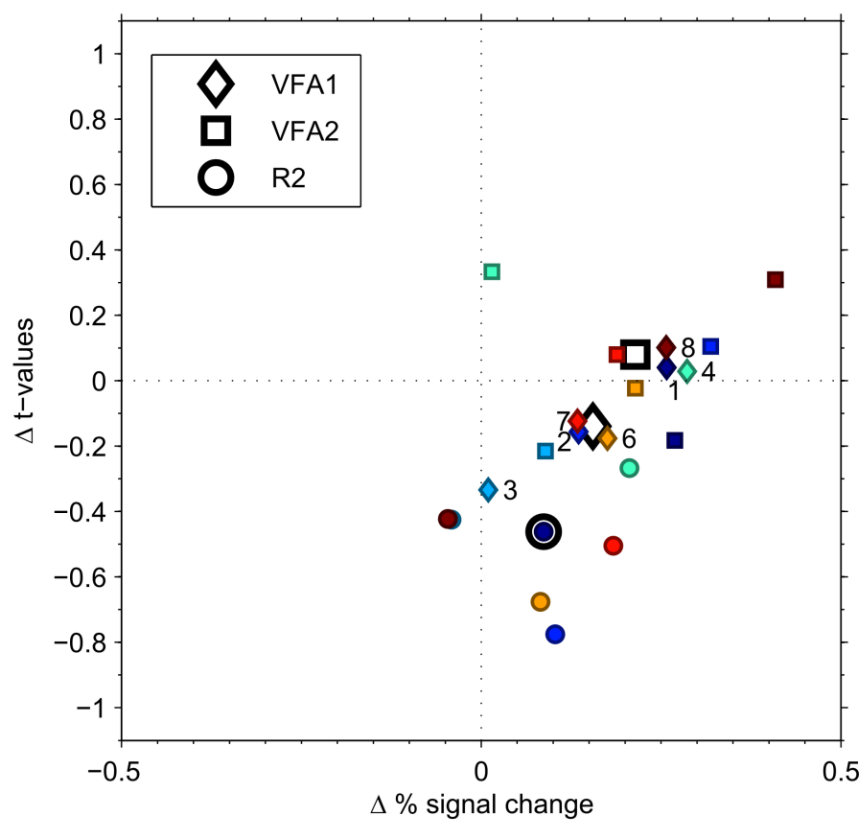

Figure 3-6: Scatterplot of differences of individual subject medians with respect to REF acquisition. Colors represent different subjects, open markers represent median across subjects.

\section{Image point-spread function}

Our method was validated using phantom and in vivo measurements. In the phantom scans we noted excellent agreement of simulations and measurements. Compared to conventional refocusing schemes, in-vivo VFA measurements exhibit significantly reduced blurring. With the VFA approach we were able to obtain a similar PSF to that of large FoV 2D SEEPI along the phase-encoding direction (Kemper et al., 2015b). In addition, the coverage of VFA2 was almost doubled compared to REF. REF is in good agreement with (Kemper et al., 2015b). Deviations stem from the longer TE and the different fitting approaches. Note that experimental imperfections in the $180^{\circ}$ refocusing pulses can be advantageous for the PSF while this is not necessarily the case for lower flip angles. This explains why in REF, the experimental blurring is smaller than theoretically expected. VFA2 exhibits slightly higher blurring than theoretically expected and compared to VFA1. This may be because of physiological noise sources introducing phase shifts 
throughout the much longer echo train, which could potentially be mitigated using more sophisticated image-reconstruction algorithms (Kim et al., 2015). Finally, constant flip angle schemes employing angles below $180^{\circ}$ (Fernandez-Seara et al., 2005) would yield a much smaller improvement over $180^{\circ}$ pulses than the presented VFA acquisitions. For $140^{\circ}$ constant refocusing flip angles we found a reduction of blurring FWHM of $8 \%$ in simulations and $3-8 \%$ in measurements (tested in four subjects), compared to $180^{\circ}$ (REF).

\section{tSNR and functional sensitivity}

The tSNR in both VFA acquisitions was smaller compared to REF. The reduction was most apparent in regions experiencing insufficient $R F$ transmission (see Figure 3-2 bottom), to which the VFA acquisitions were more sensitive than the conventional measurement. Importantly, less RF efficiency, which is typically linked to improved homogeneity (e.g. in $\mathrm{B}_{1}$ shimming approaches (Metzger et al., 2008; De Martino et al., 2012)), would be acceptable, because of the low SAR of the VFA acquisitions.

Image SNR is commonly known to be dominated by the signal intensity of k-space center (in our case proportional to $\sin \left(\alpha_{0}\right) \times \sin ^{2}\left(\alpha_{1} / 2\right)$, where $\alpha_{0}$ is the excitation flip angle and $\alpha_{1}$ the first refocusing angle) and to scale with the square root of the sampling time (depending on the number of slices and partial Fourier acquisition (Edelstein et al., 1986)). This explains the reduction of tSNR in VFA1 while the latter partly recovers TSNR in VFA2, because it employs a longer sampling time.

Higher functional signal changes were observed in both VFA acquisitions, thereby preserving the contrast-to-noise ratio and hence functional sensitivity. Higher signal changes are expected because a higher fraction of the sampled signal experiences long echo times (in spin-echo pathways) or long mixing times (in stimulated echo pathways). Thus, in both signal pathways spins experience longer diffusion times in the presence of microscopic magnetic field gradients surrounding capillaries and venules containing deoxygenated blood (dynamic averaging) (Yacoub et al., 2003; Goerke et al., 2007; Uludag et al., 2009). This mechanism increases the BOLD contrast. Differential responses from small, adjacent cortical patches or cortical layers can be clouded by image blurring reducing the contrastto-noise ratio (Yacoub et al., 2008; Kemper et al., 2015b). The reduced blurring using VFA will therefore improve the functional sensitivity in 
differential functional paradigms (when the areas are aligned with the partition direction of the imaging slab).

\section{Alternative ways to increase FoV}

For comparison, in-plane accelerated data were acquired, as an alternative approach to increase the FoV. These did not yield satisfying results. The likely reason is insufficiently distinct RF receive profiles. A different coil design might be advantageous for this application (Koning et al., 2013), allowing for reduced g-factors. Further, a high resolution receive coil grid with smaller elements might allow for acceleration along the smaller partition direction. Alternatively, independent multi-slab as well as multibanded (Larkman et al., 2001) multi-slab acquisitions have been demonstrated (Chen and Feinberg, 2013; Vu et al., 2013). These approaches allow imaging multiples of the FoV in separate locations; however, acquiring an increased, contiguous FoV might be limited by saturation effects from imperfect slab profiles and insufficiently distinct receive RF coil sensitivity profiles, for the separation of the multi-banded slabs in high spatial resolution acquisitions. A larger, contiguous FoV is preferable for applications that focus on imaging larger cortical areas. Note, however, that the VFA technique could straightforwardly be combined with in-plane accelerated imaging and/or these multi-slab approaches.

\section{Acknowledgements}

This study was supported by European Research Council (ERC) grant 269853, and the National Institute of Biomedical Imaging and Bioengineering (NIBIB) P41 EB015894. F.D.M. was funded by NWO VIDI (grant 864-13-012). 


\section{References}

Bandettini, P.A., Wong, E.C., Jesmanowicz, A., Hinks, R.S., and Hyde, J.S. (1994). Spin-Echo and Gradient-Echo Epi of Human Brain Activation Using Bold Contrast - a Comparative-Study at $1.5 \mathrm{~T}$. Nmr in Biomedicine 7, 12-20. doi: 10.1002/nbm.1940070104.

Barth, M., Meyer, H., Kannengiesser, S.A., Polimeni, J.R., Wald, L.L., and Norris, D.G. (2010). T2-weighted 3D fMRI using S2-SSFP at 7 tesla. Magn Reson Med 63, 1015-1020. doi: 10.1002/mrm.22283.

Busse, R.F., Brau, A.C., Vu, A., Michelich, C.R., Bayram, E., Kijowski, R., Reeder, S.B., and Rowley, H.A. (2008). Effects of refocusing flip angle modulation and view ordering in 3D fast spin echo. Magn Reson Med 60, 640-649. doi: 10.1002/mrm.21680.

Busse, R.F., Hariharan, H., Vu, A., and Brittain, J.H. (2006). Fast spin echo sequences with very long echo trains: design of variable refocusing flip angle schedules and generation of clinical T2 contrast. Magn Reson Med 55, 1030-1037. doi: 10.1002/mrm.20863.

Chen, L., and Feinberg, D. (Year). "Simultaneous Multi-Volume GRASE Imaging", in: Proceedings of the 21st Annual Meeting of ISMRM), 2365.

Constable, R.T., Kennan, R.P., Puce, A., Mccarthy, G., and Gore, J.C. (1994). Functional NMR imaging using fast spin echo at 1.5 T. Magn Reson Med 31, 686-690. doi: 10.1002/mrm.1910310617.

De Martino, F., Schmitter, S., Moerel, M., Tian, J., Ugurbil, K., Formisano, E., Yacoub, E., and Van De Moortele, P.-F. (2012). Spin echo functional MRI in bilateral auditory cortices at $7 \mathrm{~T}$ : An application of B-1 shimming. Neuroimage 63, 1313-1320. doi: 10.1016/j.neuroimage.2012.08.029.

Duong, T.Q., Yacoub, E., Adriany, G., Hu, X., Ugurbil, K., and Kim, S.G. (2003). Microvascular BOLD contribution at 4 and $7 \mathrm{~T}$ in the human brain: gradient-echo and spin-echo fMRI with suppression of blood effects. Magn Reson Med 49, 1019-1027. doi: 10.1002/mrm.10472.

Edelstein, W.A., Glover, G.H., Hardy, C.J., and Redington, R.W. (1986). The intrinsic signal-to-noise ratio in NMR imaging. Magn Reson Med 3, 604-618. doi: 10.1002/mrm.1910030413.

Feinberg, D.A., Harel, N., Ramanna, S., Ugurbil, K., and Yacoub, E. (2008). Sub-millimeter Single-shot 3D GRASE with Inner Volume Selection for T2 weighted fMRI applications at 7 Tesla. 16th Annual Meeting International Society for Magnetic Resonance in Medicine, 2373.

Feinberg, D.A., Hoenninger, J.C., Crooks, L.E., Kaufman, L., Watts, J.C., and Arakawa, M. (1985). Inner volume MR imaging: technical concepts 
and their application. Radiology 156, 743-747. doi: 10.1148/radiology.156.3.4023236.

Feinberg, D.A., and Oshio, K. (1991). GRASE (gradient- and spin-echo) MR imaging: a new fast clinical imaging technique. Radiology 181, 597602. doi: 10.1148/radiology.181.2.1924811.

Fernandez-Seara, M.A., Wang, Z., Wang, J., Rao, H.Y., Guenther, M., Feinberg, D.A., and Detre, J.A. (2005). Continuous arterial spin labeling perfusion measurements using single shot 3D GRASE at 3 T. Magn Reson Med 54, 1241-1247. doi: 10.1002/mrm.20674.

Goa, P.E., Koopmans, P.J., Poser, B.A., Barth, M., and Norris, D.G. (2014). BOLD fMRI signal characteristics of S1- and S2-SSFP at 7 Tesla. Frontiers in Neuroscience 8. doi: 10.3389/fnins.2014.00049.

Goense, J.B.M., and Logothetis, N.K. (2006b). Laminar specificity in monkey V1 using high-resolution SE-fMRI. Magnetic Resonance Imaging 24, 381-392. doi: 10.1016/j.mri.2005.12.032.

Goerke, U., Garwood, M., and Ugurbil, K. (2011). Functional magnetic resonance imaging using RASER. Neuroimage 54, 350-360.

Goerke, U., Van De Moortele, P.F., and Ugurbil, K. (2007). Enhanced relative BOLD signal changes in $\mathrm{T}(2)$-weighted stimulated echoes. Magn Reson Med 58, 754-762. doi: 10.1002/mrm.21369.

Griswold, M.A., Jakob, P.M., Heidemann, R.M., Nittka, M., Jellus, V., Wang, J., Kiefer, B., and Haase, A. (2002). Generalized autocalibrating partially parallel acquisitions (GRAPPA). Magn Reson Med 47, 1202-1210. doi: 10.1002/mrm.10171.

Heidemann, R.M., Ivanov, D., Trampel, R., Fasano, F., Meyer, H., Pfeuffer, J., and Turner, R. (2012). Isotropic submillimeter fMRI in the human brain at $7 \mathrm{~T}$ : combining reduced field-of-view imaging and partially parallel acquisitions. Magn Reson Med 68, 1506-1516. doi: $10.1002 / \mathrm{mrm} .24156$.

Hennig, J. (1988). Multiecho imaging sequences with low refocusing flip angles. Journal of Magnetic Resonance (1969) 78, 397-407.

Hennig, J., Nauerth, A., and Friedburg, H. (1986). RARE imaging: a fast imaging method for clinical MR. Magn Reson Med 3, 823-833. doi: $10.1002 / \mathrm{mrm} .1910030602$.

Hennig, J., Weigel, M., and Scheffler, K. (2003). Multiecho sequences with variable refocusing flip angles: optimization of signal behavior using smooth transitions between pseudo steady states (TRAPS). Magn Reson Med 49, 527-535. doi: 10.1002/mrm.10391.

Hua, J., Qin, Q., Van Zijl, P.C., Pekar, J.J., and Jones, C.K. (2013b). Wholebrain three-dimensional T2-weighted BOLD functional magnetic resonance imaging at 7 Tesla. Magn Reson Med. doi: $10.1002 / \mathrm{mrm} .25055$. 
Kemper, V.G., De Martino, F., Vu, A.T., Poser, B.A., Feinberg, D.A., Goebel, R., and Yacoub, E. (2015b). Sub-Millimeter T2 Weighted fMRI at 7 $\mathrm{T}$ : Comparison of 3D-GRASE and 2D SE-EPI. Frontiers in Neuroscience 9. doi: 10.3389/fnins.2015.00163.

Kim, H., Kim, D.H., and Park, J. (2015). Variable-flip-angle single-slab 3D GRASE imaging with phase-independent image reconstruction. Magn Reson Med 73, 1041-1052. doi: 10.1002/mrm.25223.

Koning, W., Bluemink, J.J., Langenhuizen, E.A., Raaijmakers, A.J., Andreychenko, A., Van Den Berg, C.A., Luijten, P.R., Zwanenburg, J.J., and Klomp, D.W. (2013). High-resolution MRI of the carotid arteries using a leaky waveguide transmitter and a high-density receive array at 7 T. Magn Reson Med 69, 1186-1193. doi: 10.1002/mrm.24345.

Larkman, D.J., Hajnal, J.V., Herlihy, A.H., Coutts, G.A., Young, I.R., and Ehnholm, G. (2001). Use of multicoil arrays for separation of signal from multiple slices simultaneously excited. Journal of Magnetic Resonance Imaging 13, 313-317.

Liang, X., Connelly, A., Tournier, J.D., and Calamante, F. (2014). A variable flip angle-based method for reducing blurring in 3D GRASE ASL. Phys Med Biol 59, 5559-5573. doi: 10.1088/00319155/59/18/5559.

Metzger, G.J., Snyder, C., Akgun, C., Vaughan, T., Ugurbil, K., and Van De Moortele, P.F. (2008). Local B1+ shimming for prostate imaging with transceiver arrays at 7T based on subject-dependent transmit phase measurements. Magn Reson Med 59, 396-409. doi: 10.1002/mrm.21476.

Mugler, J.P., 3rd, Epstein, F.H., and Brookeman, J.R. (1992). Shaping the signal response during the approach to steady state in threedimensional magnetization-prepared rapid gradient-echo imaging using variable flip angles. Magn Reson Med 28, 165-185. doi: 10.1002/mrm.1910280202.

Park, J., Mugler, J.P., 3rd, Horger, W., and Kiefer, B. (2007). Optimized T1weighted contrast for single-slab 3D turbo spin-echo imaging with long echo trains: application to whole-brain imaging. Magn Reson Med 58, 982-992. doi: 10.1002/mrm.21386.

Pfeuffer, J., Van De Moortele, P.-F., Yacoub, E., Shmuel, A., Adriany, G., Andersen, P., Merkle, H., Garwood, M., Ugurbil, K., and Hu, X. (2002). Zoomed Functional Imaging in the Human Brain at 7 Tesla with Simultaneous High Spatial and High Temporal Resolution. Neurolmage 17, 272-286. doi: 10.1006/nimg.2002.1103.

Pohmann, R., Speck, O., and Scheffler, K. (2015). Signal-to-noise ratio and MR tissue parameters in human brain imaging at 3, 7, and 9.4 tesla 
using current receive coil arrays. Magn Reson Med. doi: $10.1002 / \mathrm{mrm} .25677$.

Poser, B.A., and Norris, D.G. (2007). Fast spin echo sequences for BOLD functional MRI. MAGMA 20, 11-17. doi: 10.1007/s10334-006-0063$x$.

Pruessmann, K.P., Weiger, M., Scheidegger, M.B., and Boesiger, P. (1999). SENSE: sensitivity encoding for fast MRI. Magnetic resonance in medicine 42, 952-962.

Qin, Q. (2012). Point spread functions of the T2 decay in k-space trajectories with long echo train. Magn Reson Imaging 30, 11341142. doi: 10.1016/j.mri.2012.04.017.

Uludag, K., Muller-Bierl, B., and Ugurbil, K. (2009). An integrative model for neuronal activity-induced signal changes for gradient and spin echo functional imaging. Neuroimage 48, 150-165. doi: 10.1016/j.neuroimage.2009.05.051.

Van De Moortele, P.F., Auerbach, E.J., Olman, C., Yacoub, E., Ugurbil, K., and Moeller, S. (2009). T1 weighted brain images at 7 Tesla unbiased for Proton Density, $\mathrm{T} 2 *$ contrast and RF coil receive B1 sensitivity with simultaneous vessel visualization. Neuroimage 46, 432-446. doi: 10.1016/j.neuroimage.2009.02.009.

Vu, A., Feinberg, D., Harel, N., Ugurbil, K., and Yacoub, E. (2013). Diagonal multi-slab inner volume 3D GRASE imaging for high resolution T2 weighted fMRI. Proceedings of the 21st Annual Meeting of ISMRM 21, 2364.

Yacoub, E., Duong, T.Q., Van De Moortele, P.F., Lindquist, M., Adriany, G., Kim, S.G., Ugurbil, K., and Hu, X. (2003). Spin-echo fMRI in humans using high spatial resolutions and high magnetic fields. Magn Reson Med 49, 655-664. doi: 10.1002/mrm.10433.

Yacoub, E., Harel, N., and Ugurbil, K. (2008). High-field fMRI unveils orientation columns in humans. Proc Natl Acad Sci U S A 105, 10607-10612. doi: 10.1073/pnas.0804110105.

Yacoub, E., Shmuel, A., Logothetis, N., and Ugurbil, K. (2007). Robust detection of ocular dominance columns in humans using Hahn Spin Echo BOLD functional MRI at 7 Tesla. Neuroimage 37, 1161-1177. doi: 10.1016/j.neuroimage.2007.05.020.

Yacoub, E., Van De Moortele, P.F., Shmuel, A., and Ugurbil, K. (2005). Signal and noise characteristics of Hahn SE and GE BOLD fMRI at $7 \mathrm{~T}$ in humans. Neuroimage 24, 738-750. doi: 10.1016/j.neuroimage.2004.09.002.

Zaitsev, M., Hennig, J., and Speck, O. (2004). Point spread function mapping with parallel imaging techniques and high acceleration factors: fast, robust, and flexible method for echo-planar imaging distortion 
Chapter 3

correction. Magn Reson Med 52, 1156-1166. doi: 10.1002/mrm.20261. 


\section{Chapter 4}

\subsection{T anatomical and functional}

investigation of human primary and

secondary visual cortex

Corresponding publication: Kemper, Valentin G.; De Martino, Federico; Emmerling, Thomas; Yacoub, Essa; Goebel, Rainer. "9.4 T anatomical and functional investigation of human primary and secondary visual cortex" (in preparation). 


\begin{abstract}
Ultra-high field functional magnetic resonance imaging (fMRI) employing the $T_{2}$ weighted blood oxygenation dependent (BOLD) effect provide sufficient specificity and sensitivity to sample cortical structures at the submillimeter scale. Anatomical MRI at ultra-high field affords novel applications because of stronger contrasts and higher resolution. Thus far, little experience exists for human fMRI at field strengths above 7 Tesla. This study provides preliminary evidence for the potential of field strengths higher than 7T in the delineation of functional and anatomical properties of visual cortical areas. We employed a human 9.4 $\mathrm{T}$ scanner to obtain $\mathrm{T}_{2}{ }^{*}$ and $T_{2}$ weighted BOLD functional data and high resolution $T_{1}$ and $T_{2}{ }^{*}$ weighted anatomical images. We delineated visual areas V1 and V2 using population receptive field mapping and gradient-echo echo planar imaging. Within these retinotopically defined cortical regions (corresponding to Brodmann areas 17 and 18, respectively) we analyzed the anatomical contrast from $\mathrm{T}_{2}{ }^{*}$ weighted data $(0.35 \mathrm{~mm}$ isotropic resolution) and found distinct differences in their cortical depth dependent contrast profiles. Further, we report preliminary results of ocular preference in early visual cortex from monocular stimulation and $T_{2}$ weighted inner-volume single-shot 3DGRASE acquisitions. Our preliminary analysis shows that high resolution $\mathrm{T}_{2}$ weighted functional data allow detecting functional differences in organization of ocular dominance between V1 and V2. Primary visual cortex exhibits higher functional specificity to either eye and the specificity was spatially organized in one of two subjects. The organization in V1 was consistent across cortical depth forming ocular dominance columns.
\end{abstract}




\section{Introduction}

Blood oxygenation level dependent (BOLD, Ogawa et al., 1990) functional magnetic resonance imaging ( $\mathrm{fMRI}$ ) allows investigating human brain function non-invasively and has greatly advanced the knowledge about its organization. One of the early applications of fMRI was the functional segregation of individual visual areas, such as primary visual cortex (V1) and secondary visual cortex (V2). To date, more than twenty visual areas (Silver and Kastner, 2009; Wandell and Winawer, 2011) can be identified by the means of retinotopic mapping (DeYoe et al., 1996; Engel et al., 1997) which defines the preferred polar angle and eccentricity of a given cortical location. Recently, population receptive field (pRF) mapping has been introduced to obtain the receptive field size of neuronal populations and more accurate estimates of preferred retinotopic location (Dumoulin and Wandell, 2008).

A characterization of early visual areas can also be obtained using anatomical contrast in vivo. Early visual cortex, V1-V3 (Brodmann areas 17 and 18), is known to be highly myelinated and this information can be mapped using cortical myelin mapping techniques that use (combinations of) $T_{1}$ weighted, $T_{2}$ and/or $T_{2}{ }^{*}$ weighted imaging (Glasser and Van Essen, 2011; De Martino et al., 2014; Lutti et al., 2014). Overall myelination allows the distinction between early and higher level visual cortices as validated with post mortem probabilistic atlases (Abdollahi et al., 2014). However, the average myelination across cortical depth does not allow the distinction of areas within early visual cortex (e.g. V1 from V2). To do so myelin information needs to be extracted in a cortical depth dependent manner by $\mathrm{T}_{2}{ }^{*}$ weighted magnitude or phase imaging or susceptibility weighted imaging (Duyn et al., 2007; Fukunaga et al., 2010; Budde et al., 2011; Budde et al., 2014a) as laminar profiles are clearly different both in terms of myelin and cytoarchitecture (Ramón y Cajal, 1899; Brodmann, 1909; Vogt and Vogt, 1919; Nieuwenhuys, 2013). Good agreement between cortical depth dependent anatomical contrast in V1 and V2 and retinotopically defined borders has been demonstrated (Bridge et al., 2005; Sánchez-Panchuelo et al., 2012).

At the functional level V1 can be characterized on the basis of the preference to stimulus features other than retinotopic position albeit at a smaller spatial scale. Besides preference with respect to right or left eye 
(ocular dominance columns, ODCs, Hubel and Wiesel, 1965; 1977; Grinvald et al., 1991), preference to spatial and temporal frequency (Shoham et al., 1997), and to orientation (Blasdel and Salama, 1986; Bonhoeffer and Grinvald, 1991) have been observed in animals. ODCs form stripes of varying width and with bifurcations and other irregularities (LeVay et al., 1985). They tend to be oriented orthogonally to the V1/V2 border, where they end abruptly (Ts'o et al., 1990). In human V1, ODCs have been shown in post-mortem samples using cytochrome oxidase staining in monocular blind (Horton and Hedley-Whyte, 1984; Horton et al., 1990; Adams et al., 2007). The mean width of a single column is approximately $0.8-1.1 \mathrm{~mm}$ (Adams et al., 2007; Yacoub et al., 2007).

ODCs have been mapped in-vivo using ultra-high field (i.e. $\geq 7$ Tesla) fMRI. Early experiments targeting ODCs mostly used segmented gradient-echo echo-planar imaging (EPI) techniques fMRI (Menon et al., 1997; Dechent and Frahm, 2000; Cheng et al., 2001; Goodyear and Menon, 2001). At ultrahigh field, the $T_{2}$ weighted (spin-echo) BOLD effect is less dominated by large veins which drain larger portions of gray matter than $\mathrm{T}_{2}{ }^{*}$ weighted (gradient-echo) BOLD. Thus, spin-echo BOLD is more specific to changes on a small spatial scale, i.e. the functional point spread function is narrower. This has been confirmed in experiments and simulations (Duong et al., 2003; Yacoub et al., 2005; Yacoub et al., 2007; Uludag et al., 2009). Spinecho based imaging approaches have yielded more precise maps of ODCs despite the lower contrast-to-noise ratio of $T_{2}$ weighted $f M R I$ in single conditions without differential contrasts (Yacoub et al., 2007). In either case, $\mathrm{T}_{2}$ weighted or $\mathrm{T}_{2}{ }^{*}$ weighted imaging, researchers have used thick slices with high in-plane resolution positioned tangentially to small, flat pieces of calcarine sulcus. For this reason, ODCs have not been studied systematically in extended portions of V1 or its surroundings using fMRI. 3D inner-volume gradient- and spin-echo (3D-GRASE, Feinberg and Oshio, 1991; Feinberg et al., 2008) overcomes the limitation of the anisotropic resolution and could therefore be used to map the functional organization also in folded cortical regions outside V1 (Zimmermann et al., 2011; De Martino et al., 2013). It yields a $T_{2}$ weighted contrast similar to the segmented inner-volume $T_{2}$ weighted BOLD imaging approach chosen in imaging ODCs and orientation columns in V1 (Yacoub et al., 2008). Moreover, it was demonstrated to yield better specificity than full-field-of- 
view spin-echo EPI (due to the shorter EPI echo train duration of 3D-GRASE (Kemper et al., 2015b)).

Both anatomical and functional studies targeting fine-scaled structures have been greatly facilitated by the continuous improvements of spatial resolution as well as sensitivity at higher magnetic field strengths. As 7 Tesla magnets become increasingly available to neuroscientists, higher field strengths such as $9.4 \mathrm{~T}$ bear the potential to push the limits even further (Pohmann et al., 2015). Thus far, only few anatomical or functional studies have been performed at 9.4 T human systems (Budde et al., 2011; Ehses et al., 2013; Budde et al., 2014a; Budde et al., 2014b; Ehses et al., 2014; Bause et al., 2015; Scheffler and Ehses, 2015) due to the limited accessibility and technical difficulties. The challenges are similar as at lower field strength, e.g. $7 \mathrm{~T}$, but more pressing. The RF wavelength is shorter at higher field (higher Larmor frequency) leading to increased power deposition and increased spatial excitation inhomogeneity. Static field inhomogeneity due to susceptibility differences is enhanced, transverse relaxation times $\left(T_{2}, T_{2}{ }^{*}\right)$ are shorter, and the longitudinal relaxation time is longer. Only after overcoming these hindrances, the increased SNR of 9.4 T can be exploited. Variable flip angle 3D-GRASE can meet these challenges. $\mathrm{T}_{2}{ }^{*}$ contamination is minimized by the use of inner-volume selection in conjunction with fast gradient switching of a dedicated head gradient system. $T_{2}$ blurring and SAR can be reduced by the application of a variable refocusing flip-angle approach (Liang et al., 2014; Kemper et al., 2015c). Therefore, this study sets out to extend the experience in high-resolution functional and anatomical imaging of human visual cortex. We report pRF mapping using standard gradient-echo EPI, high-resolution $\mathrm{T}_{2}{ }^{*}$ weighted anatomical imaging of cortical depth dependent signals in V1 and V2, and mapping of ocular dominance volumetrically at isotropic resolution using $\mathrm{T}_{2}$ weighted 3D-GRASE.

\section{Methods}

Two healthy female volunteers (mean age $25 \pm 1$ ) were scanned in two sessions on a 9.4 T MRI (Siemens, Erlangen, Germany) equipped with a head-only gradient coil (max. $80 \mathrm{mT} / \mathrm{m}$ at $400 \mathrm{mT} / \mathrm{m} / \mathrm{s}$ slew rate) and an 8channel transmit 24-channel receive surface coil (Life Services, Minneapolis, MN, USA) covering the posterior part of the subjects head. A static, non-subject-specific $\mathrm{B}_{1}^{+}$phase shim was used throughout the 
measurements, after it had been validated that this approach generated sufficiently efficient and homogeneous RF fields in early visual areas in various subjects (Kemper et al., 2015a) using DREAM $B_{1}$ mapping (Nehrke and Börnert, 2012; Tse et al., 2014). Only the global scaling factor (reference voltage) was adjusted to the individual subjects after acquiring a pre-saturation approach based flip angle map. SAR supervision was maintained by imposing the maximum local SAR level observed in simulations as global RF power deposition limits.

Second order shimming was applied to homogenize the $B_{0}$ magnetic field in a manually drawn region of interest in early visual areas using a custommade dual-echo field mapping GRE sequence and MATLAB (The MATHWORKS Inc., Natick, MA, USA) routines.

\section{$\underline{\text { Visual stimulation }}$}

Visual stimulation was provided using a projector and a frosted screen near the subject's head, seen via a tilted mirror. Population receptive field mapping was performed using randomly moving bars (Senden et al., 2014; Goebel, 2015). Horizontal, vertical, and oblique full contrast flickering checkerboards bars (flickering rate $7.5 \mathrm{~Hz}$ ) were presented at 12 different positions spanning $0.63^{\circ}$ visual angle each with maximum eccentricity of $7.5^{\circ}$ (square-shaped). During anatomical acquisitions, subjects watched animated cartoon movies.

For binocular eye stimulation, subjects wore anaglyph glasses (Lee filters, Andover, Hampshire, UK; red \#026, cyan \#116) throughout the entire second experimental session. The stimulus colors were adjusted such that the eyes could be stimulated individually using the pass-band optical wavelength of the filter plates. Subjects were instructed to fixate a central fixation dot, while full field concentric flickering checkerboard patterns were presented. The fixation dot was moved $2.5^{\circ}$ upwards, such that more of the lower visual field was stimulated. A block design switching between right and left eye every 48 seconds was chosen (Yacoub et al., 2007). In the beginning and end 24 seconds of gray screen were presented the same luminance as the non-stimulated eyes perceived. Seven runs of 7:12 min duration each were performed. Figure 4-1 shows a drawing of the binocular stimulation set-up. 

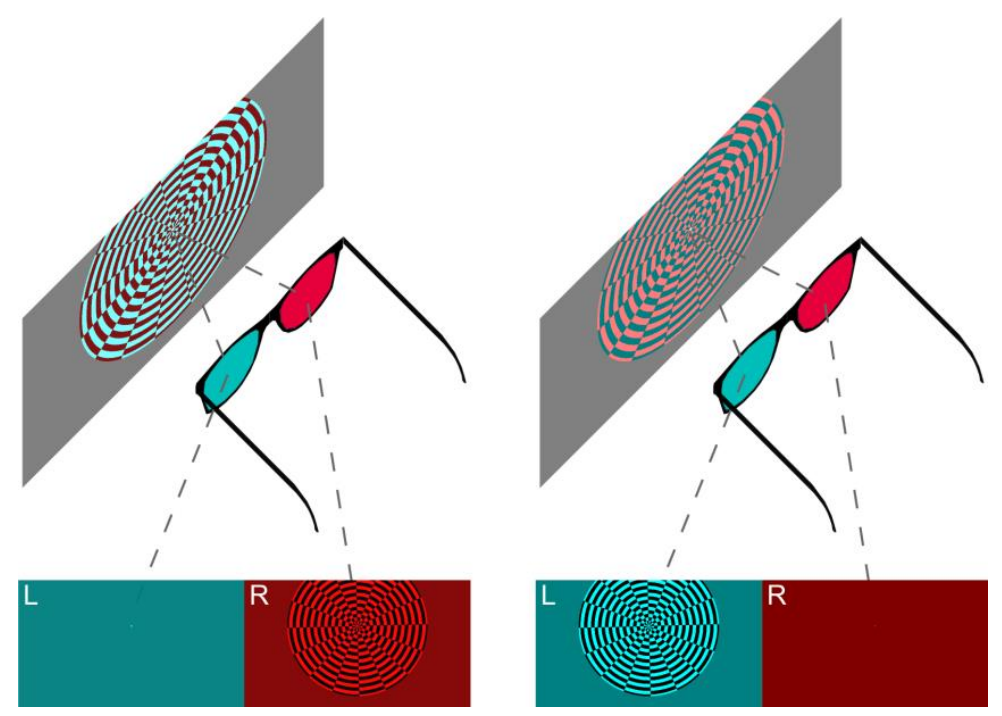

Figure 4-1: Cartoon display of binocular stimulation using anaglyph glasses.

\section{Imaging pulse sequences}

Anatomical reference images were acquired with a $T_{1}$ weighted MPRAGE sequence $\left(T E / T I / T R=2.5 / 1200 / 3600 \mathrm{~ms}\right.$; nominal flip angle $=4^{\circ}$; matrix size $=384 \times 384 \times 256$; GRAPPA acceleration factor 3, partial Fourier factor 6/8 (partition direction); total duration 8:54 min.). In addition, a proton density weighted MPRAGE without the inversion module (identical imaging parameters except $T R=1620 \mathrm{~ms}$ and total duration $=4: 01 \mathrm{~min}$.) was acquired to correct for transmission and receive coil biases (Van de Moortele et al., 2009). TI and flip angle were adapted from $7 \mathrm{~T}$ protocols based on literature values for $T_{1}$ and proton density in white and gray matter at 7 and 9.4 T and the equations in Deichmann et al. (2000) and Marques et al. (2010).

Population receptive fields mapping was performed using a gradient-echo EPI sequence $(1.05 \mathrm{~mm}$ isotropic nominal resolution; $\mathrm{TE} / \mathrm{TR}=14.6 / 2000 \mathrm{~ms}$; in-plane FoV $105 \times 105 \mathrm{~mm}$; 54 coronal slices; nominal flip angle $70^{\circ}$; echo spacing $0.64 \mathrm{~ms}$; GRAPPA 2, partial Fourier 7/8; $13: 20$ min. duration, 3 repetitions)

Anatomical high resolution $\mathrm{T}_{2}{ }^{*}$ weighted images were acquired using a 2D GRE sequence with limited FoV $0.35 \mathrm{~mm}$ isotropic nominal resolution $\left(0.043 \mathrm{~mm}^{3}\right) ; \mathrm{TE} / \mathrm{TR}=20 / 1500 \mathrm{~ms}$; in-plane FoV $100 \times 100 \mathrm{~mm}$; 48 slices; 
nominal flip angle; $70^{\circ}$.; bandwidth $80 \mathrm{~Hz} /$ Pixel; no parallel imaging, no partial Fourier; 7:09 min. duration, 6 averages).

Ocular dominance imaging was performed using a high-resolution innervolume 3D-GRASE sequence (TE/TR $=30.85 / 2000 \mathrm{~ms}$; in-plane FoV 22.4×105 mm; 12 slices; nominal flip angles (90-107-60-56-53-54-54-58-70107) ${ }^{\circ}$; echo spacing $0.75 \mathrm{~ms}$; partial Fourier 6/8 (partition direction); 8:00 min. duration, 7 repetitions). Images were acquired at a nominal isotropic resolution of $0.7 \mathrm{~mm}$, and were then interpolated to $0.35 \mathrm{~mm}$ isotropic resolution using the complex, uncombined coil data. This was done in order not to introduce any resolution losses due to co-registration or motioncorrection. Refocusing flip angles were adjusted based on extended phase graph theory simulations to yield reduced across-slice blurring of estimated 2.3 voxels (Kemper et al., 2015c).

\section{Data analysis}

Functional data analysis was performed using BrainVoyager QX 2.8.4 (Brain Innovation, Maastricht, the Netherlands) and MATLAB routines. Preprocessing of the population receptive field data included slice-time correction, 3D-motion correction, and high-pass filtering. Additionally, data was distortion-corrected using acquisitions with reversed phase encoding direction and the COPE plug-in (v0.5) in BrainVoyager (Andersson et al., 2003; Fritz et al., 2014). The three runs were averaged and analyzed to generate pRF estimates for each voxel. Model time courses for pRFs of receptive field positions between $\pm 7.5^{\circ}$ eccentricity in horizontal and vertical position and size (Gaussian full width at half maximum) between $0.2^{\circ}$ and $10^{\circ}$ were calculated, to which the experimental data was compared to find the best match (highest correlation). Functional data were co-registered to the intra-session reference anatomical data using positional and edge information. Anatomical alignment across sessions was used to align all (functional and anatomical) data to the anatomical reference image of the second (ocular dominance) session. The resulting maps for voxel-wise retinotopic eccentricity, polar angle, and pRF size were projected onto the surface representations of the hemispheres (see below). Visual areas V1 and V2 were delineated by the reversal of polar angle at the vertical meridian. Delineation was possible down to an eccentricity of approximately $2^{\circ}$. Below this limit, the polar angle did not yield sufficient information. 
Preprocessing of the ocular dominance functional data only included 3Dmotion correction and linear trend removal. Individual runs were aligned with one-another and co-registered with the $\mathrm{T}_{1}$ weighted anatomical acquisition using positional information. Fine-tuning of the alignment was performed manually using image edge information. A general linear model was calculated using the right and left eye condition predictors after convolution with a standard 2-gamma-function hemodynamic response function. Additionally, motion parameters were used as confound predictors (three translational and three rotational per run). As a measure for eye preference, we calculated selectivity, $s$, as the normalized relative contribution between the BOLD responses to the right and left eye $(s=$ $\left.\left(B_{R}-b_{L}\right) /\left(B_{R}+b_{L}\right)\right)$. The following three criteria were used to identify voxels with reasonable characteristics to analyze eye preference on: 1 ) Voxels had to have a small eccentricity in the population receptive field measurement below $5^{\circ}$ visual angle. This limit was chosen because subjects reported ocular rivalry "creeping in" from peripheral visual field areas, such that, at times, subjects would perceive the color-shaded gray background in the non-stimulated eye more pronounced than the checkerboards in the stimulated eye. Such effects are more likely to occur at higher eccentricity (Blake et al., 1992). 2) BOLD responses to right or left eye individually had to be significant $(p<0.001)$ and both positive to rule out areas of negative BOLD responses. 3) BOLD signal changes above a threshold of $7 \%$ were discarded as likely macrovascular contributions. The group of voxels that fulfilled the criteria 1-3 (small eccentricity, small BOLD \% signal change, significant activation for right or left eye individually) was considered to have sufficient signal quality for ocular dominance to be observed if present.

Permuting the spatial distribution of the selectivity index $s$ allowed us to assess the null distribution of the median absolute $s$ (median $(|s|))$ in V1 and V2 and thus the significance of the difference between V1 and V2. Spatial permutations were created by swapping at random $\mathrm{N}$ voxels from V1 and V2 (where $\mathrm{N}$ was determined as $70 \%$ of the smaller number of voxels fulfilling criteria 1-3 in V1 and V2). This procedure was repeated 1000 times to obtain the empirical null distribution of selectivity values against which the selectivity of V1 and V2 were compared.

The anatomical high-resolution $\mathrm{T}_{2}{ }^{*}$ weighted images were co-registered to one-another and then averaged. Prior to that, the images were cropped 
such that no fold-in in phase-encoding direction was present. The averaged dataset was divided by a strongly spatially smoothed version of it (Garcia, 2010) this step retained local anatomical contrast but eliminated lowspatial-frequency image inhomogeneity stemming from the RF coil profiles. The brain was masked for this step based on intensity so that the local average was not reduced at the edges. The resulting image was coregistered with the MPRAGE anatomical reference.

The $T_{1}$ weighted anatomical reference images were divided by the proton density weighted images. Further, low-spatial frequency inhomogeneity correction was applied. Automatic image segmentation of white matter (WM) and gray matter (GM) was performed and refined manually. Finally, the hemispheres were separated and individual surface representations were created from the mid-GM sheet.

\section{Results}

\section{Population receptive fields}

Figure 4-2 summarizes the results of the population receptive field mapping. Early visual areas could be readily identified. Multiple polar angle reversal, which mark the transitions from V1 to V2 and from V2 to V3, are readily observable in the surface maps, both ventrally and dorsally. Additionally, at least hV4, LO1, and LO2 can be distinguished.

\section{Anatomical cortical depth profiles}

Figure 4-3 shows an exemplary transversal cut along with the $T_{1}$ weighted reference and an overlay of the segmentation from subject 1 . The Stria of Gennari was visible in the $\mathrm{T}_{2}{ }^{*}$ weighted high resolution anatomical images in both subjects. Average cortical depth profiles of the $\mathrm{T}_{2}{ }^{*}$ weighted anatomical contrast were generated after grouping the data according to the functional delineation from the population receptive field mapping. The results for both participants are shown in Figure 4-4. The average V1 profile exhibits a marked reduction of signal intensity in the middle of the cortical depth, whereas the V2 profile is rather continuously increasing.

$\rightarrow$ Figure 4-2: Population receptive field results of both subjects and both hemispheres (columns). Surface maps from left to right: Eccentricity, polar angles, prf size, and delineation of areas V1 and V2. Only the dorsal part of V2 was labeled. 

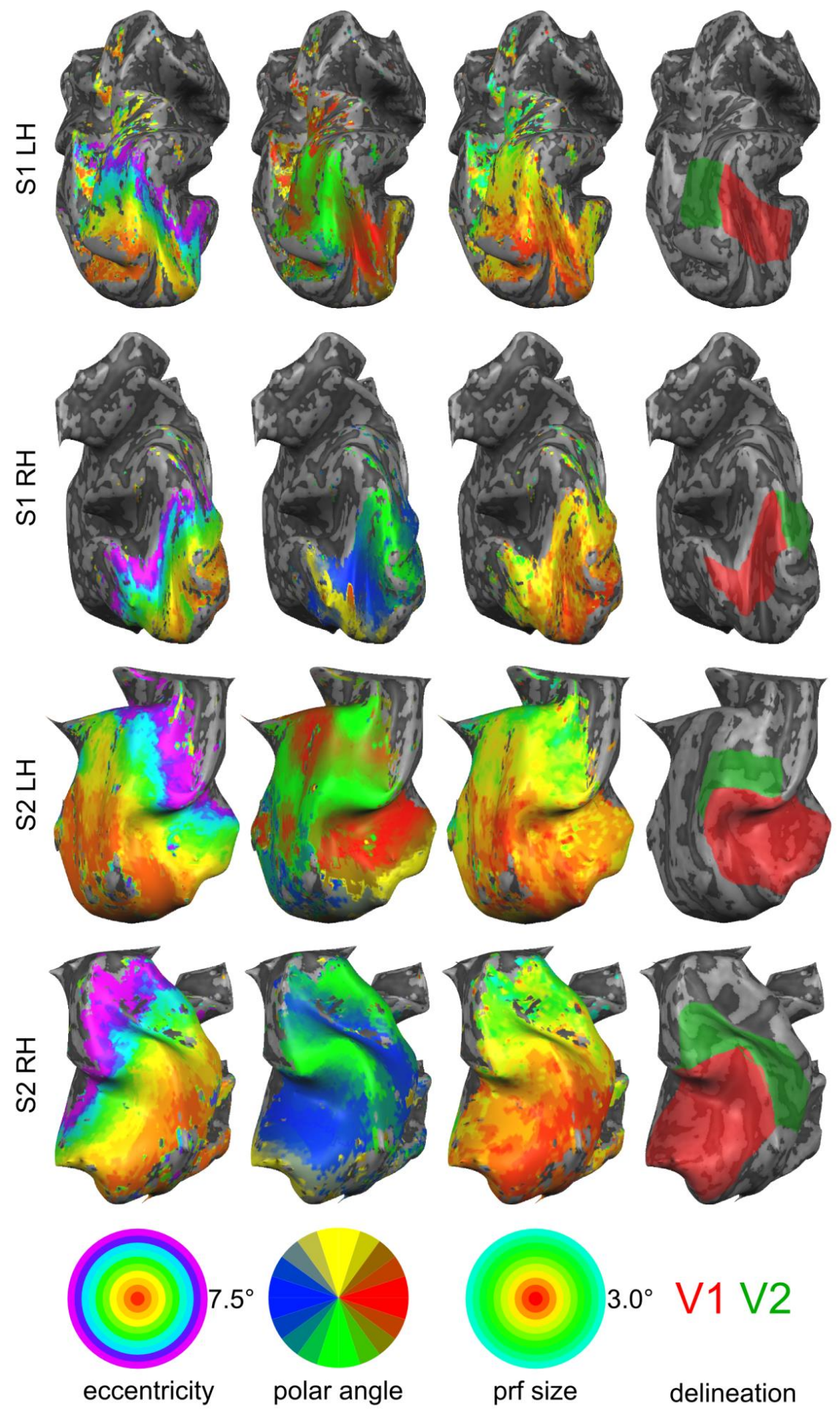

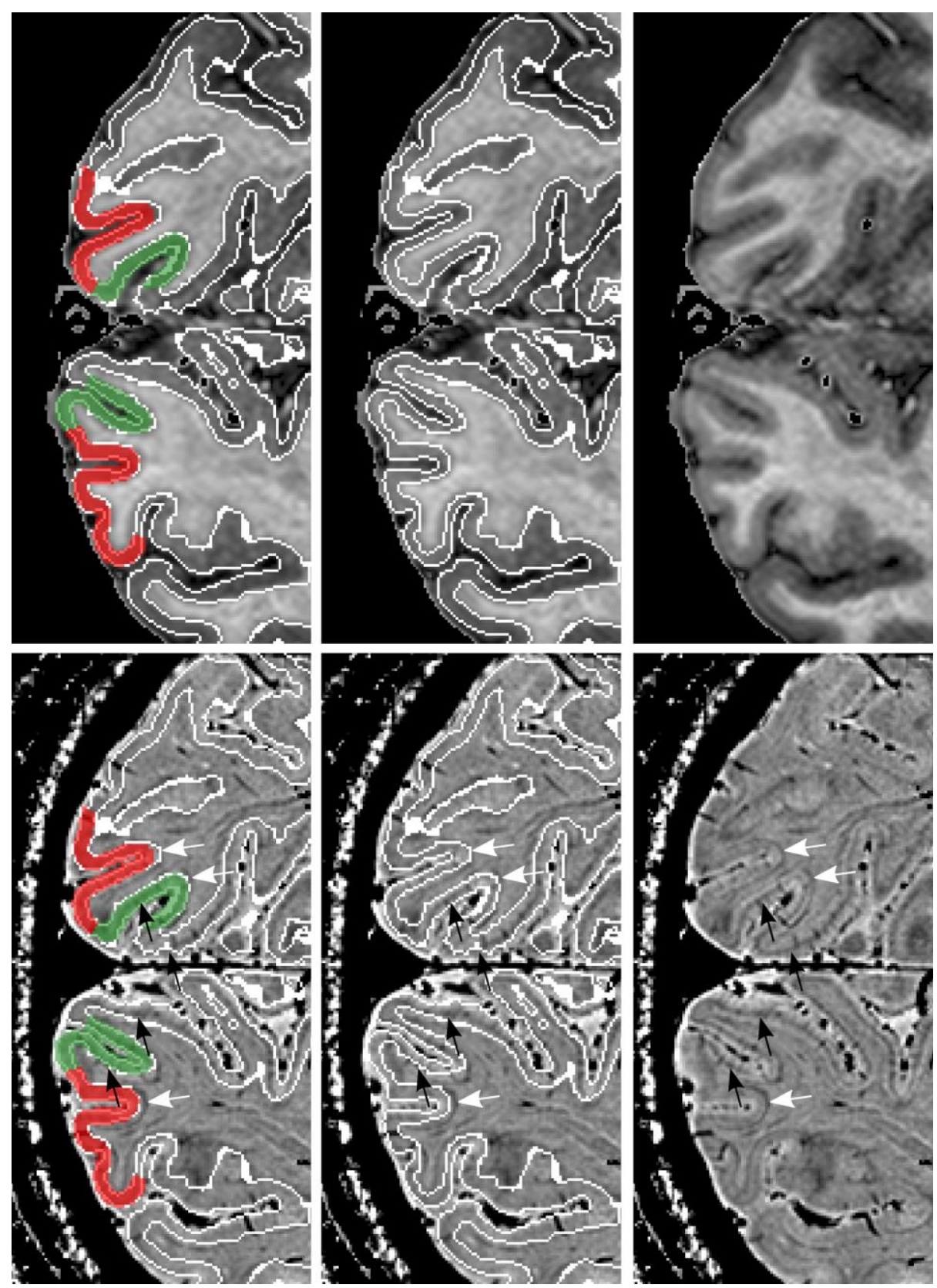

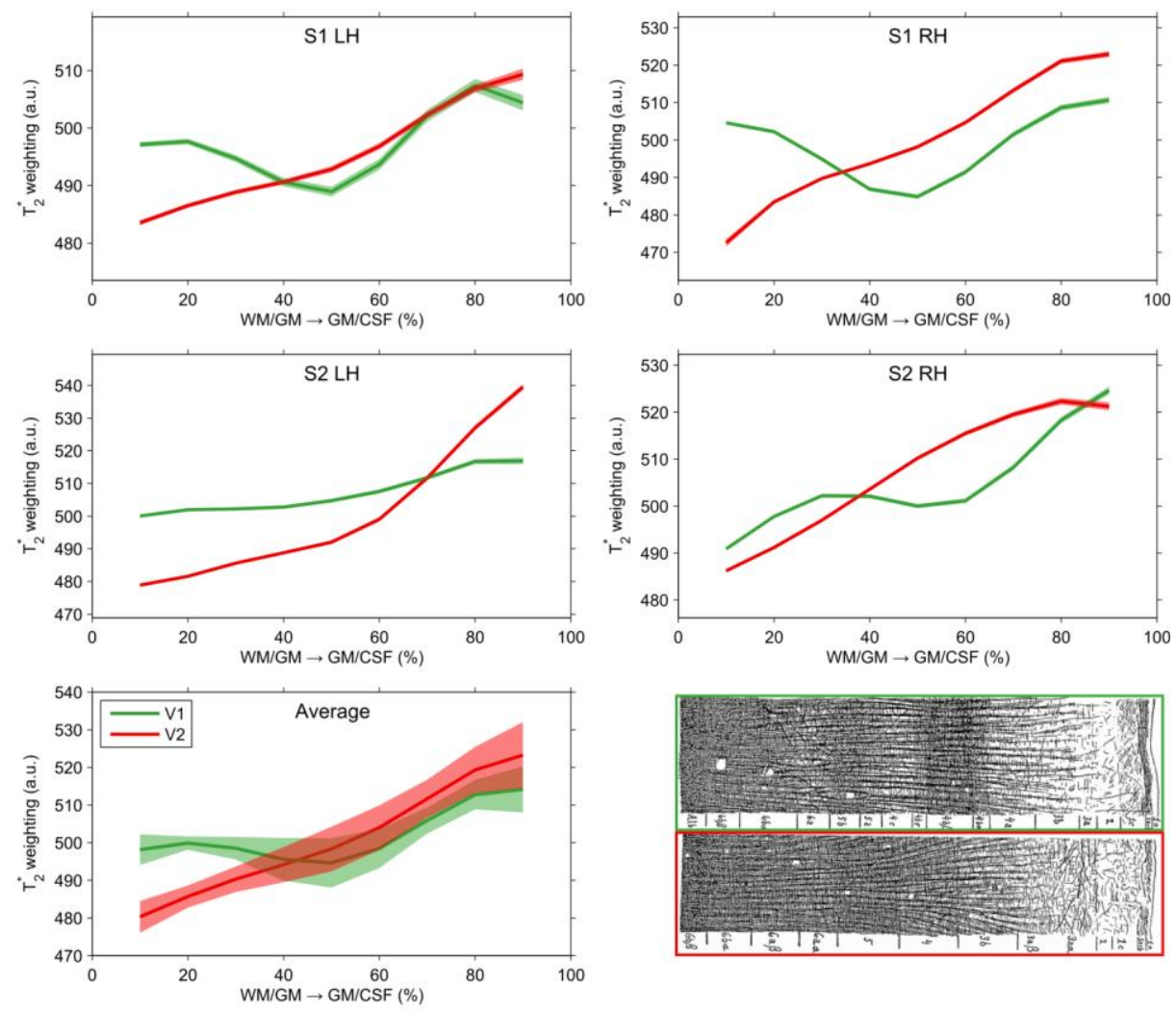

Figure 4-4: Cortical depth profiles of high resolution $\mathrm{T}_{2}{ }^{*}$ weighted anatomical data sampled within V1 (green) and V2 (red) as determined from the population receptive field measurements. Both subjects and both hemispheres are shown as well as their average (average \pm std. error). For comparison, myelin stains were taken from Nieuwenhuys (2013) Fig. 29.

\&Figure 4-3: Transversal cuts through anatomical data. Left column: $\mathrm{T}_{1}$ weighted MPRAGE divided by proton density weighted MPRAGE. Right column: $\mathrm{T}_{2}{ }^{*}$ weighted GRE data. Middle row: Overlaid boundary of cortical gray matter (white lines). Bottom row: Visual areas V1 (green) and V2 (red) as determined from the population receptive field measurements overlaid. Note the clear appeance of the Stria of Gennari in the $\mathrm{T}_{2}{ }^{*}$ weighted image (black arrows) and dark lines just underneath the gray matter in white matter (white arrows). 


\section{Ocular dominance}

Figure 4-5 displays the selectivity as extracted from the 3D GRASE data at middle cortical depth on surface maps. A patchy organization can be observed in all hemispheres, but only little preference for the right eye is registered in subject 1 . Selectivity for both eyes is similarly distributed in V1 of subject 2 .

The bar plots in Figure 4-5 show the ratio of the subset of selective voxels (right vs. left significant, student's t-test $p<0.05$, uncor.) within the group of voxels fulfilling criteria 1-3. This analysis was performed in the volume space. When the chance level of criterion 3 (significant activation for right or left eye individually) is reduced (lower $p$, higher $t$-value), the ratios increase as expected, because significant differences between left and right eye are more easily detected in more robust voxels. In all hemispheres, the ratio of selective to non-selective voxels is higher in V1 than in V2 across a wide range of chance levels. Figure 4-6 shows the results of the spatial permutation tests. The selectivity was found to be significantly different between the retionotopically defined V1, V2, and the permuted region of interest in all hemispheres.

Figure 4-7 displays volumetric representations of high resolution cortical depth sampling grids from a region of interest within V1 of subject 2 . For both hemispheres, maps of selectivity and of binarized ocular dominance are shown. Selectivity maps were only masked using criteria 1-3, but no significance thresholding for the difference right versus left was used. The binary ocular dominance maps were thresholded using the same statistical values as in (Yacoub et al., 2007). A high degree of consistency across cortical depth is observed in both types of maps and in both hemispheres. The maps are split up to facilitate comparison across cortical depth. 

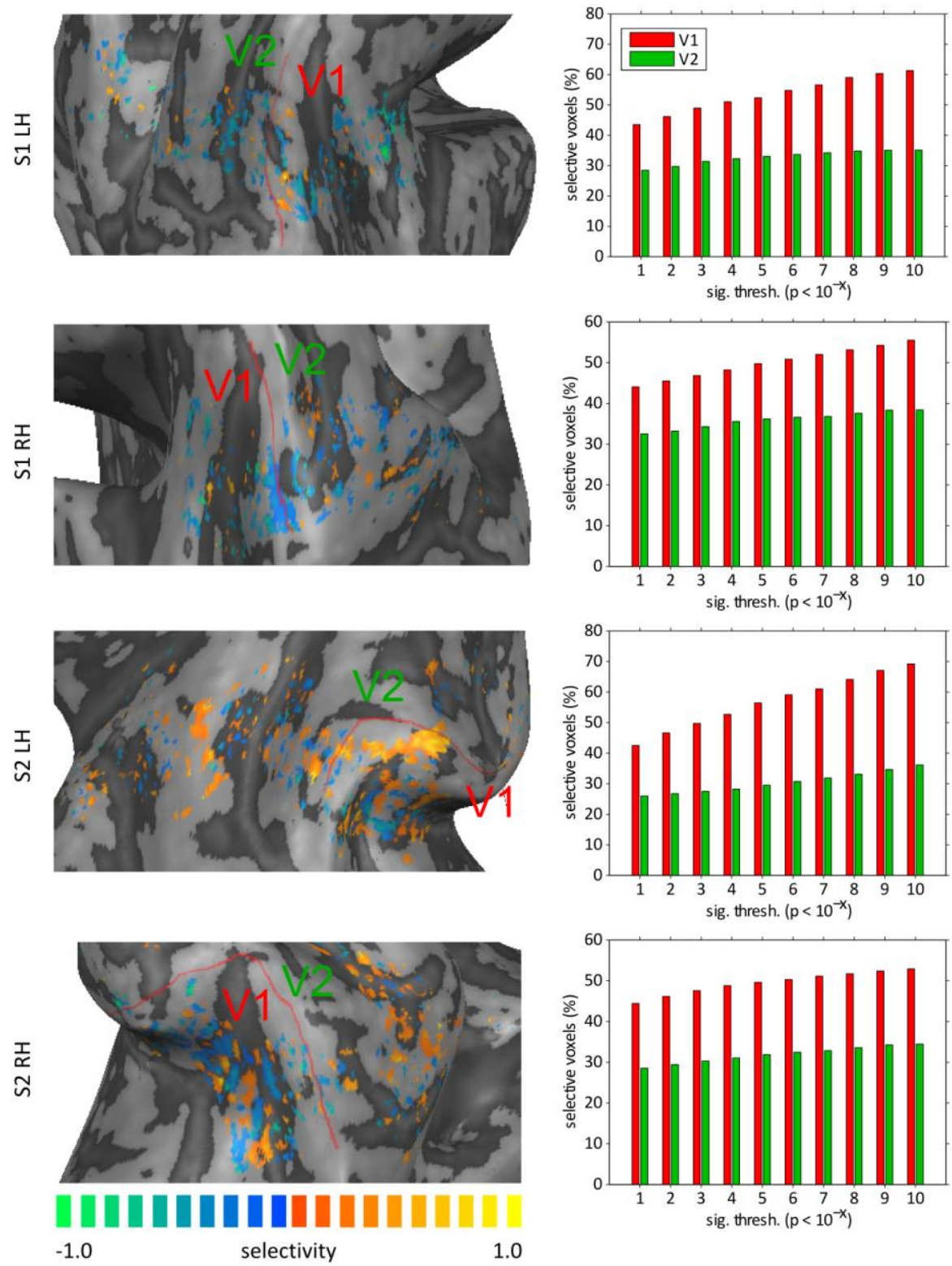

Figure 4-5: Surface maps of selectivity index for right versus left eye $\left(\left(B_{R}-B_{L}\right) /\left(B_{R}+B_{L}\right)\right)$. Right side: bar plots for the same hemispheres of significant eye preference within group of voxels fulfilling criteria 1-3 (see text for details). 

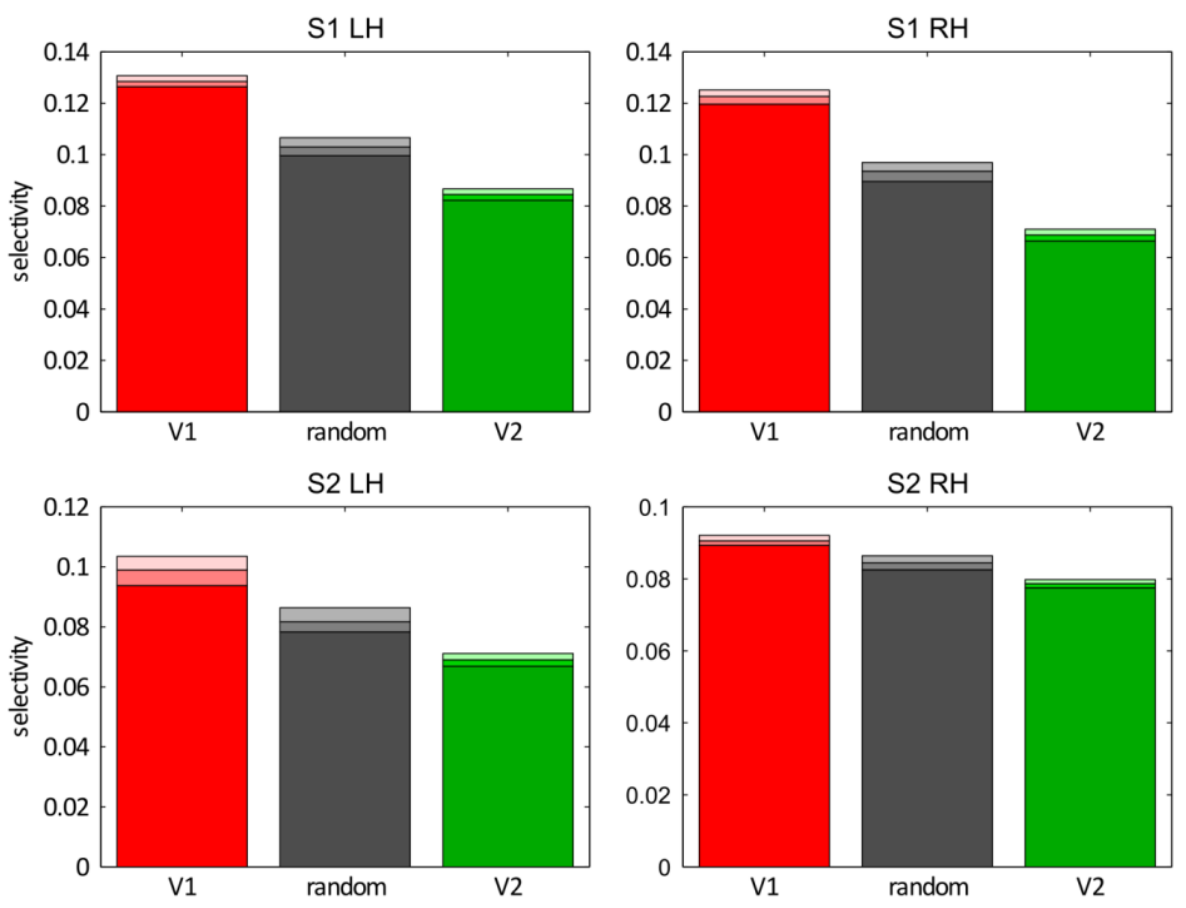

Figure 4-6: Spatial permutation test results. Bar graph of median absolute selectivity index $s$ when sampled from $V 1, V 2$, or from both ROls at random. Bar colors indicate medians and $95 \%$ confidence intervals as obtained from the repeated random subset drawings from the ROIs (1000 repetitions).

\section{Discussion}

The present study investigated human early visual cortex anatomically and functionally at high spatial resolution using 9.4 T MRI. Few functional studies have thus far been performed in-vivo in humans at 9.4 $\mathrm{T}$ (see introduction for references) due to the technical challenges associated with it. In our study, the limitation to early visual areas allowed for the use of an $\mathrm{RF}$ coil with transmit and receive sensitivity confined to a small region for better $\mathrm{B}_{1}{ }^{+}$efficiency and high SNR. In fact, SAR was at such a low level that it did not impede the temporal efficiency of the imaging protocols or the achievable flip angles. In the case of the $T_{2}$ weighted acquisitions, this is also due to the variable flip angle 3D-GRASE approach. 


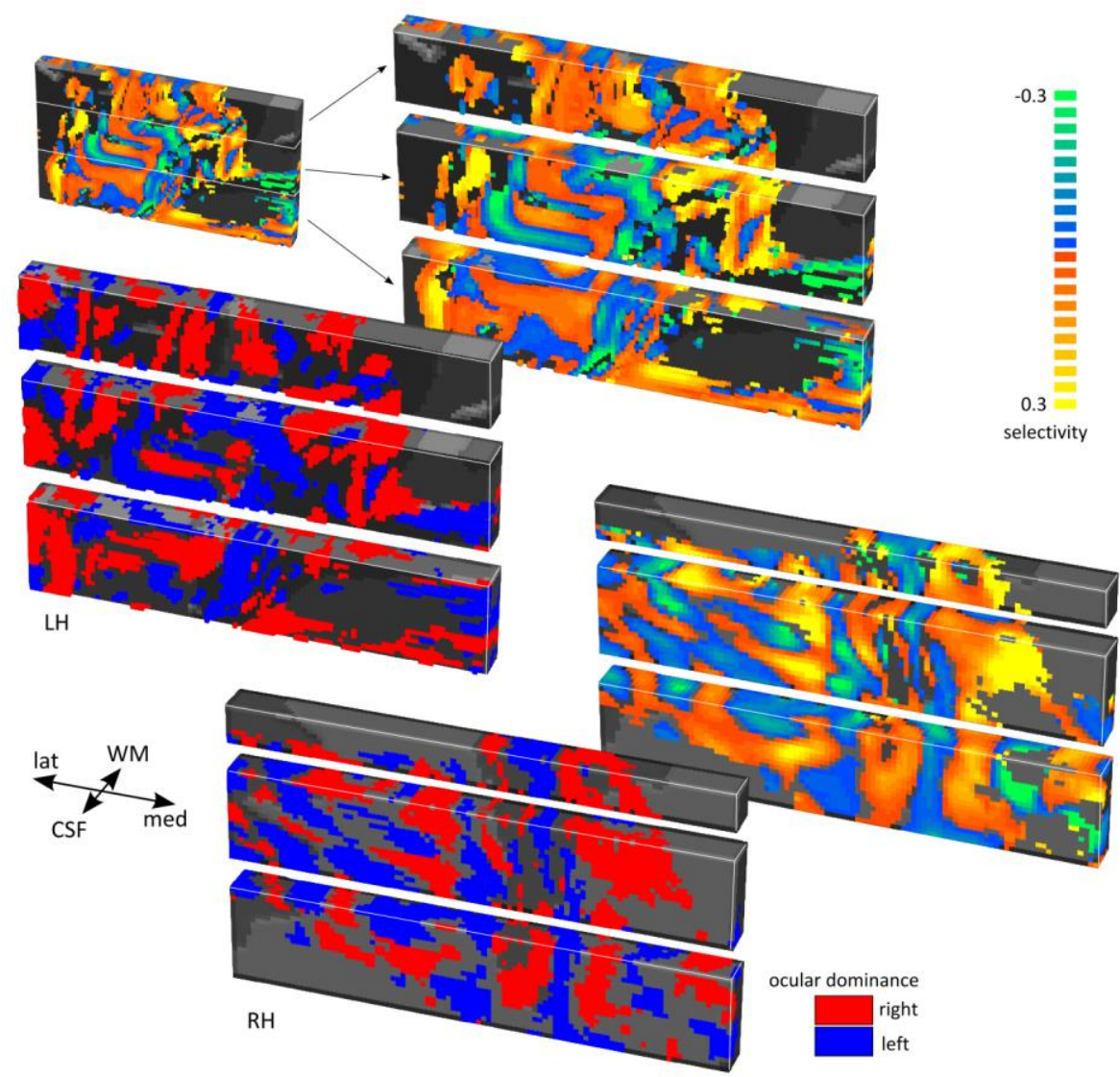

Figure 4-7: 3D representation of selectivity maps and binary ocular dominance maps from high resolution cortical depth sampling grids in V1 of subject 2. Maps are shown between 10 and $90 \%$ cortical depth. The orientation is similar to surface maps in Figure 4-5 (lat: lateral; med: medial; CSF cerebrospinal fluid; WM: white matter). For the binary maps, the same statistical thresholds were used as in (Yacoub et al., 2007).

A routine visual experiment (i.e. retinotopic mapping) was successfully conducted at a relatively high resolution (1.05 $\mathrm{mm}$ isotropic) and yielded excellent results. Further, we demonstrated the achievement of high quality anatomical images. These were obtained at $0.35 \mathrm{~mm}$ nominal isotropic resolution isotropic $\left(0.043 \mathrm{~mm}^{3}\right)$, which has rarely been reported previously in-vivo (Budde et al., 2014a; Forstmann et al., 2014). Cortical depth profiles obtained from these $\mathrm{T}_{2}{ }^{*}$ weighted anatomical data reflected differences in the laminarization between $\mathrm{V} 1$ and $\mathrm{V} 2$ as defined by the 
population receptive field retinotopic measurements. We also present preliminary findings of ocular dominance employing 3D-GRASE for $0.7 \mathrm{~mm}$ nominal isotropic resolution $\left(0.34 \mathrm{~mm}^{3}\right)$. In both subjects, the specificity of response to either eye was different between $\mathrm{V} 1$ and $\mathrm{V} 2$. In one of the two subjects, ocular dominance maps in V1 were characterized by spatially clustered responses that alternated in preference for either eye when moving tangentially to the cortex. This organization was consistent across cortical depth suggestive of ocular dominance columns.

\section{Population receptive fields}

We presented population receptive field mapping at $1.05 \mathrm{~mm}$ isotropic nominal resolution using a gradient-echo EPI sequence. The purpose of these measurements was to delineate V1 and V2 accurately and precisely, which we succeeded in due to the high SNR of the $9.4 \mathrm{~T}$. Our results are in agreement with the literature results from lower field strengths, e.g. (Goebel et al., 1998; Dumoulin and Wandell, 2008; Silver and Kastner, 2009; Winawer et al., 2010; Wandell and Winawer, 2011). The presented surface maps have a small, uncharted area at the occipital poles. This could be the consequence of two factors. First, imperfections in the EPI data due to acquisition strategy (i.e. distortions because of the left-right phaseencoding direction) may degrade the signal in this region. Studies typically choose anterior-posterior phase-encoding, which yields more benign image distortions in this anatomical location. Our particular choice is justified in order to shorten the echo train duration and exploit the RF coil geometry for better SNR. Second, because the location of the uncharted area coincides with the foveal confluence, where the cortical magnification factor is extreme, attention effects and micro-saccades in conjunction with the fact that the fixation dot was static might impede the pRF mapping (Schira et al., 2009; Wandell and Winawer, 2011).

\section{Anatomical cortical depth profiles}

Our high resolution $\mathrm{T}_{2}{ }^{*}$ weighted anatomical data revealed a cortical depthspecific difference in contrast between V1 and V2, and the results are in agreement with the literature (Duyn et al., 2007; Fukunaga et al., 2010; Budde et al., 2011). A dark band in middle cortical depth can be identified by visual inspection in all hemispheres. This signature seizes to exist beyond the retinotopically defined boundary between V1 and V2. This dark band likely corresponds to the Stria of Gennari. $\mathrm{T}_{2}{ }^{*}$ weighted images are 
sensitive to differences in iron concentration, which has been demonstrated to be closely co-localized with myelin (Fukunaga et al., 2010). Further, Lee et al. (2010) have shown that endogenous deoxyhemoglobin has a negligible effect, which allows the conclusion that the dark band is not related to the denser capillary bed in the middle cortical vascular layer 3.

When analyzing the average cortical depth profile throughout V1 and V2 broadening or blurring of the profiles takes place because of local differences in cortical depth, curvature, segmentation, and alignment inaccuracies. The laminar depth profiles in Figure 4-4 appear fuzzier than in previous publications because the data was averaged across a large region of interest, while localized profiles have been presented in publications of other groups. Alignment inaccuracies could be (virtually) eliminated if the gray/white matter segmentation could be performed on the data itself, or at least a dataset with the same distortions (Clare and Jezzard, 2001; Renvall et al., 2014). However, the image contrast in the present data was not sufficient, likely being the cause for the dissimilar V1 profile in the left hemisphere of subject 2 . An additional acquisition with $T_{1}$ weighting with otherwise same imaging modalities would be beneficial. Alternatively, even a combined $\mathrm{T}_{1}-\mathrm{T}_{2}{ }^{*}$ weighted acquisition with varied TEs, TRs and/or Tls would be an option. Further, it has been demonstrated (Waehnert et al., 2014) that cortical layers follow cortical thickness more closely when assessed in an isovolumetric approach than in the approach based on the Laplace equation chosen here (Jones et al., 2000; Zimmermann et al., 2011). How much this change in "laminar" assignment would affect our average profiles will be matter of future investigations. The application of prospective motion correction might further increase the spatial resolution (Zaitsev et al., 2006; Callaghan et al., 2015) by reducing intra-run motion. Eventually, obtaining high image quality that allows classifying whether a small cortical patch belongs to V1, V2, or other cortical areas would be desirable, as has been done with histological data (Annese et al., 2004) and post-mortem diffusion weighted data (Bastiani et al., 2015). A border between V1 and V2 defined in such an approach would be more accurate than curvature-based (Hinds et al., 2008) or post-mortem atlas based approaches (Wohlschläger et al., 2005; Hinds et al., 2009; Wilms et al., 2010) and potentially more reliable than visually identified borders. It has to be noted that the V1/V2 border identified based on retinotopy and 
cortical depth dependent myelination have been shown to be in good agreement, although small deviations exist (Bridge et al., 2005; SánchezPanchuelo et al., 2012).

\section{Ocular dominance}

The ocular dominance measurements using 3D-GRASE with variable flip angle refocusing revealed that the selectivity in V1 was significantly higher than in V2. This suggests that fMRI is able to detect functional differences between these two areas on the basis of eye preference. In one subject the spatial organization of selectivity alternated between both eyes (subject 2) resembling the patterns that have been reported in previous studies (Menon et al., 1997; Cheng et al., 2001; Goodyear and Menon, 2001; Yacoub et al., 2007), and this organization was consistent across cortical depth. For the first subject instead we found an overall bias for the left eye. This could have been caused by the different eye dominance of the subjects. Subject 1 was left eye dominant (as measured from behavioral performance). If the experimental stimuli were not balanced sufficiently but had a bias towards the left eye, the representation of the left eye may have been enhanced in subject 1 maps. Such an imbalance of stimulation might be caused by the application of anaglyph glasses (for the lack of a monocular stimulation device in the narrow 9.4 $\mathrm{T}$ scanner bore), or the stimulation paradigm, which, however, closely followed the paradigm employed in earlier, successful studies (Yacoub et al., 2007).

Previous fMRI studies of ocular dominance have mostly ignored responses in areas other than V1 by masking (Cheng et al., 2001) or were limited spatially to V1 (Menon et al., 1997; Goodyear and Menon, 2001; Yacoub et al., 2007). We investigated eye preference also in V2. Despite the significant difference in overall selectivity between V1 and V2, our data showed regions with high selectivity to either eye outside of V1. Hubel and Wiesel (1965) reported that a majority of the investigated neurons in cat V2 had a medium to strong preference for the contralateral eye, in fact more pronounced than in V1. However, while neurons in V1 cluster spatially according to ocular dominance in ODCs and develop more lateral connections to neurons with the same eye preference (Katz et al., 1989; Malach et al., 1993; Yoshioka et al., 1996), in V2, neither seems to be the case (Matsubara et al., 1987; Adams et al., 2007). The fact that ODCs end abruptly at the V1/V2 boundary has been observed in optical imaging (Ts'o 
et al., 1990) and post-mortem cytochrome oxidase staining studies (Horton et al., 1990; Adams et al., 2007). The observed stripy pattern of cytochrome oxidase in V2 has a different spatial frequency and is associated with different stimulus properties than ocular input.
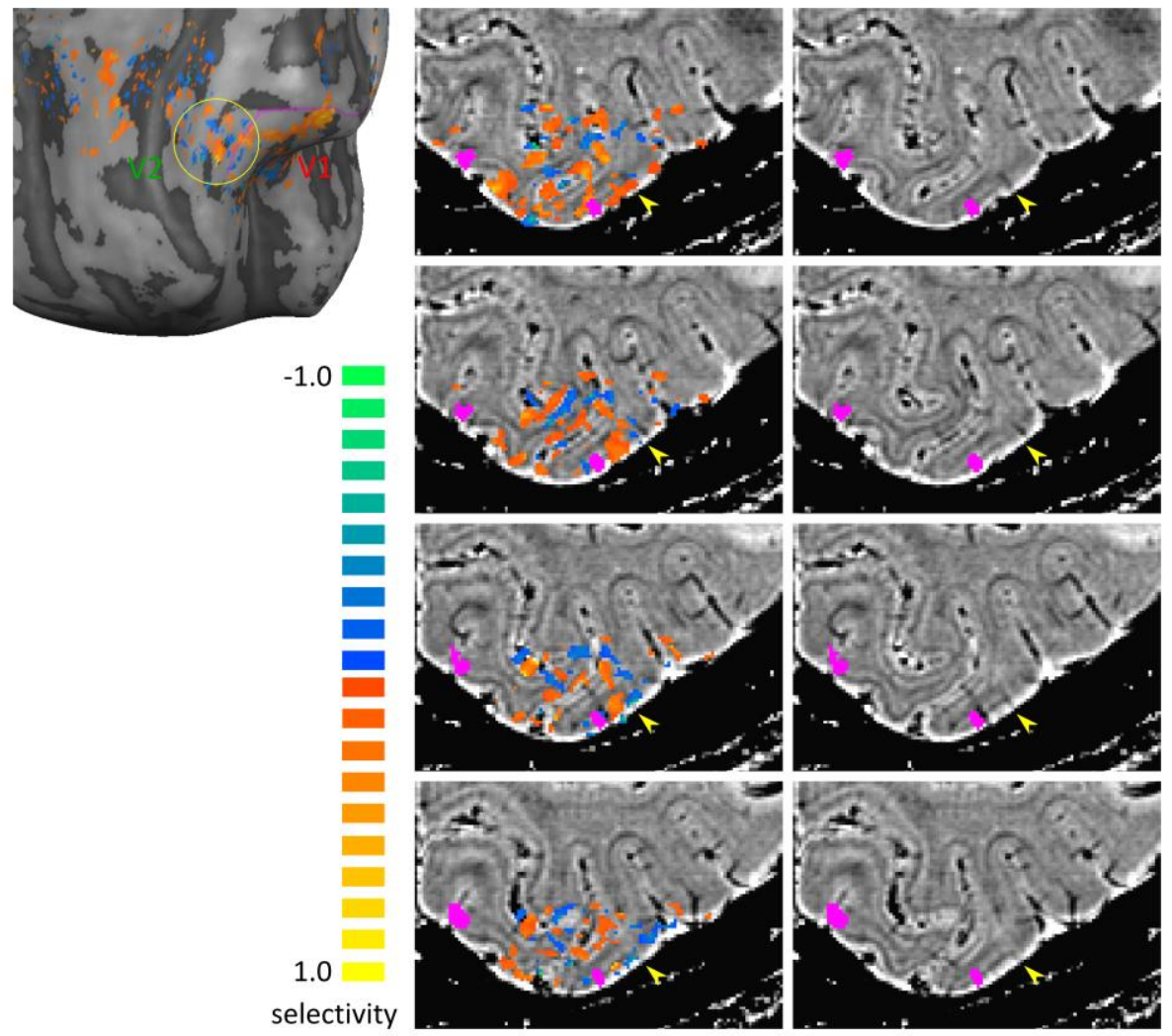

Figure 4-8: Observed mismatch between ocular dominance pattern and V1/V2 boundary as defined by the pRF mapping and accordance with high resolution $\mathrm{T}_{2}{ }^{*}$ weighted anatomical data. Left: Surface map of S2 RH (lateral view). Consecutive transversal cuts (top to bottom: superior to inferior position) with superimposed V1/V2 border from pRF mapping (magenta) with (middle column) and without (right column) overlay of selectivity. The yellow arrowheads indicate the visually identified end of the Stria of Gennari representing the anatomical boundary between V1 and V2.

The expected orthogonal organization of ODCs with respect to the V1/V2 border could not be confirmed based on our data. We did, however, notice a patch of orthogonal ODCs on the side of $\mathrm{V} 2$ as defined by the pRF results 
(see Figure 4-8). In closer inspection we found that these coincided with areas, which still revealed the V1 typical cortical depth profile in the $\mathrm{T}_{2}{ }^{*}$ weighted anatomical data. This suggests a close spatial correspondence of the anatomical definition of $\mathrm{V} 1$ and the presence of ODCs and an erroneous definition of the V1/V2 border based on the GE-EPI based retinotopy. Although preliminary, our results show the potential of having three independent functional and anatomical markers of V1 and V2.

Methodologically, the ocular dominance measurements continue our effort of high isotropic resolution $\mathrm{T}_{2}$ weighted BOLD fMRI. As such, we succeeded in exploiting the higher signal strength afforded by the $9.4 \mathrm{~T}$ scanner to increase the spatial resolution as compared to previous $7 \mathrm{~T}$ experiments using 3D-GRASE or GE-EPI. The fast switching dedicated head gradient coil, high SNR RF coil optimized for occipital cortex, and the variable flip angle 3D-GRASE sequence allowed us to obtain fMRI data of unique quality at such high resolution.

\section{Acknowledgements}

The authors would like to thank Dr. Desmond Tse, Dr. Benedikt Poser, and Dr. Christopher Wiggins for their support in the data acquisition. This study was supported by European Research Council (ERC) grant 269853, and the National Institute of Biomedical Imaging and Bioengineering (NIBIB) P41 EB015894. F.D.M. was funded by NWO VIDI (grant 864-13-012). 


\section{References}

Abdollahi, R.O., Kolster, H., Glasser, M.F., Robinson, E.C., Coalson, T.S., Dierker, D., Jenkinson, M., Van Essen, D.C., and Orban, G.A. (2014). Correspondences between retinotopic areas and myelin maps in human visual cortex. Neuroimage 99, 509-524. doi: 10.1016/j.neuroimage.2014.06.042.

Adams, D.L., Sincich, L.C., and Horton, J.C. (2007). Complete pattern of ocular dominance columns in human primary visual cortex. Journal of Neuroscience 27, 10391-10403. doi: 10.1523/jneurosci.292307.2007.

Andersson, J.L., Skare, S., and Ashburner, J. (2003). How to correct susceptibility distortions in spin-echo echo-planar images: application to diffusion tensor imaging. Neuroimage 20, 870-888. doi: 10.1016/s1053-8119(03)00336-7.

Annese, J., Pitiot, A., Dinov, I., and Toga, A. (2004). A myelo-architectonic method for the structural classification of cortical areas. Neuroimage 21, 15-26.

Bastiani, M., Oros-Peusquens, A.M., Seehaus, A., Brenner, D., Möllenhoff, K., Celik, A., Felder, J., Bratzke, H., Shah, N.J., Galuske, R., Goebel, R., and Roebroeck, A. (2015). "Automatic classification of human cortical layers and areas using high resolution diffusion MRI," in Multi-scale brain connectivity with diffusion MRI (phD thesis).).

Bause, J., Ehses, P., Mirkes, C., Shajan, G., Scheffler, K., and Pohmann, R. (2015). Quantitative and functional pulsed arterial spin labeling in the human brain at $9.4 \mathrm{t}$. Magnetic Resonance in Medicine.

Blake, R., O'shea, R.P., and Mueller, T. (1992). Spatial zones of binocular rivalry in central and peripheral vision. Visual neuroscience 8, 469478.

Blasdel, G.G., and Salama, G. (1986). Voltage-sensitive dyes reveal a modular organization in monkey striate cortex. Nature 321, 579585.

Bonhoeffer, T., and Grinvald, A. (1991). Iso-orientation domains in cat visual cortex are arranged in pinwheel-like patterns. Nature 353, 429-431.

Bridge, H., Clare, S., Jenkinson, M., Jezzard, P., Parker, A.J., and Matthews, P.M. (2005). Independent anatomical and functional measures of the V1/V2 boundary in human visual cortex. Journal of Vision 5, 1.

Brodmann, K. (1909). Vergleichende Lokalisationslehre der Groshirnrinde. Leipzig: Barth.

Budde, J., Shajan, G., Hoffmann, J., Ugurbil, K., and Pohmann, R. (2011). Human imaging at $9.4 \mathrm{~T}$ using $\mathrm{T}(2)^{*}{ }_{-}$, phase-, and susceptibility- 
weighted contrast. Magn Reson Med 65, 544-550. doi: 10.1002/mrm.22632.

Budde, J., Shajan, G., Scheffler, K., and Pohmann, R. (2014a). Ultra-high resolution imaging of the human brain using acquisition-weighted imaging at 9.4T. Neuroimage 86, 592-598. doi: 10.1016/j.neuroimage.2013.08.013.

Budde, J., Shajan, G., Zaitsev, M., Scheffler, K., and Pohmann, R. (2014b). Functional MRI in human subjects with gradient-echo and spinecho EPI at 9.4 T. Magnetic Resonance in Medicine 71, 209-218.

Callaghan, M.F., Josephs, O., Herbst, M., Zaitsev, M., Todd, N., and Weiskopf, N. (2015). An evaluation of prospective motion correction (PMC) for high resolution quantitative MRI. Frontiers in Neuroscience 9, 97. doi: 10.3389/fnins.2015.00097.

Cheng, K., Waggoner, R.A., and Tanaka, K. (2001). Human ocular dominance columns as revealed by high-field functional magnetic resonance imaging. Neuron 32, 359-374. doi: 10.1016/s08966273(01)00477-9.

Clare, S., and Jezzard, P. (2001). Rapid T(1) mapping using multislice echo planar imaging. Magn Reson Med 45, 630-634.

De Martino, F., Moerel, M., Xu, J., Van De Moortele, P.F., Ugurbil, K., Goebel, R., Yacoub, E., and Formisano, E. (2014). High-Resolution Mapping of Myeloarchitecture In Vivo: Localization of Auditory Areas in the Human Brain. Cereb Cortex. doi: 10.1093/cercor/bhu150.

De Martino, F., Zimmermann, J., Muckli, L., Ugurbil, K., Yacoub, E., and Goebel, R. (2013). Cortical depth dependent functional responses in humans at 7T: improved specificity with 3D GRASE. PLoS One 8, e60514. doi: 10.1371/journal.pone.0060514.

Dechent, P., and Frahm, J. (2000). Direct mapping of ocular dominance columns in human primary visual cortex. Neuroreport 11, 32473249.

Deichmann, R., Good, C.D., Josephs, O., Ashburner, J., and Turner, R. (2000). Optimization of 3-D MP-RAGE sequences for structural brain imaging. Neuroimage 12, 112-127. doi: 10.1006/nimg.2000.0601.

Deyoe, E.A., Carman, G.J., Bandettini, P., Glickman, S., Wieser, J., Cox, R., Miller, D., and Neitz, J. (1996). Mapping striate and extrastriate visual areas in human cerebral cortex. Proceedings of the National Academy of Sciences 93, 2382-2386.

Dumoulin, S.O., and Wandell, B.A. (2008). Population receptive field estimates in human visual cortex. Neuroimage 39, 647-660. doi: 10.1016/j.neuroimage.2007.09.034. 
Duong, T.Q., Yacoub, E., Adriany, G., Hu, X., Ugurbil, K., and Kim, S.G. (2003). Microvascular BOLD contribution at 4 and $7 \mathrm{~T}$ in the human brain: gradient-echo and spin-echo fMRI with suppression of blood effects. Magn Reson Med 49, 1019-1027. doi: 10.1002/mrm.10472.

Duyn, J.H., Van Gelderen, P., Li, T.-Q., De Zwart, J.A., Koretsky, A.P., and Fukunaga, M. (2007). High-field MRI of brain cortical substructure based on signal phase. Proceedings of the National Academy of Sciences of the United States of America 104, 11796-11801. doi: 10.1073/pnas.0610821104.

Ehses, P., Bause, J., Shajan, G., and Scheffler, K. (2014). Efficient generation of $\mathrm{T}^{*}$-weighted contrast by interslice echo-shifting for human functional and anatomical imaging at 9.4 Tesla. Magnetic Resonance in Medicine.

Ehses, P., Budde, J., Shajan, G., and Scheffler, K. (2013). T2-weighted BOLD fMRI at 9.4 T using a S2-SSFP-EPI sequence. 21st Annual Meeting of ISMRM 21, 0414.

Engel, S.A., Glover, G.H., and Wandell, B.A. (1997). Retinotopic organization in human visual cortex and the spatial precision of functional MRI. Cerebral cortex 7, 181-192.

Feinberg, D.A., Harel, N., Ramanna, S., Ugurbil, K., and Yacoub, E. (2008). Sub-millimeter Single-shot 3D GRASE with Inner Volume Selection for T2 weighted fMRI applications at 7 Tesla. 16th Annual Meeting International Society for Magnetic Resonance in Medicine, 2373.

Feinberg, D.A., and Oshio, K. (1991). GRASE (gradient- and spin-echo) MR imaging: a new fast clinical imaging technique. Radiology 181, 597602. doi: 10.1148/radiology.181.2.1924811.

Forstmann, B.U., Keuken, M.C., Schafer, A., Bazin, P.-L., Alkemade, A., and Turner, R. (2014). Multi-modal ultra-high resolution structural 7Tesla MRI data repository. Scientific data 1.

Fritz, L., Mulders, J., Bremann, H., Peters, J., Bastiani, M., Roebroeck, A., Andersson, J.L., Ashburner, J., Weiskopf, N., and Goebel, R. (Year). "Comparison of EPI distortion correction methods at 3T and 7T", in: OHBM Annual Meeting).

Fukunaga, M., Li, T.-Q., Van Gelderen, P., De Zwart, J.A., Shmueli, K., Yao, B., Lee, J., Maric, D., Aronova, M.A., Zhang, G., Leapman, R.D., Schenck, J.F., Merkle, H., and Duyn, J.H. (2010). Layer-specific variation of iron content in cerebral cortex as a source of MRI contrast. Proceedings of the National Academy of Sciences of the United States of America 107, 3834-3839. doi: 10.1073/pnas.0911177107. 
Garcia, D. (2010). Robust smoothing of gridded data in one and higher dimensions with missing values. Computational statistics \& data analysis 54, 1167-1178.

Glasser, M.F., and Van Essen, D.C. (2011). Mapping human cortical areas in vivo based on myelin content as revealed by T1-and T2-weighted MRI. The Journal of Neuroscience 31, 11597-11616.

Goebel, R. (2015). "Functional Organization of the Primary Visual Cortex," in Brain Mapping: An Encyclopedic Reference, ed. A.W. Toga. Academic Press: Elsevier), 287-291.

Goebel, R., Khorram-Sefat, D., Muckli, L., Hacker, H., and Singer, W. (1998). The constructive nature of vision: direct evidence from functional magnetic resonance imaging studies of apparent motion and motion imagery. Eur J Neurosci 10, 1563-1573.

Goodyear, B.G., and Menon, R.S. (2001). Brief visual stimulation allows mapping of ocular dominance in visual cortex using fMRI. Human Brain Mapping 14, 210-217. doi: 10.1002/hbm.1053.

Grinvald, A., Frostig, R.D., Siegel, R.M., and Bartfeld, E. (1991). Highresolution optical imaging of functional brain architecture in the awake monkey. Proceedings of the National Academy of Sciences 88, 11559-11563.

Hinds, O., Polimeni, J.R., Rajendran, N., Balasubramanian, M., Amunts, K., Zilles, K., Schwartz, E.L., Fischl, B., and Triantafyllou, C. (2009). Locating the functional and anatomical boundaries of human primary visual cortex. Neuroimage 46, 915-922.

Hinds, O.P., Rajendran, N., Polimeni, J.R., Augustinack, J.C., Wiggins, G., Wald, L.L., Rosas, H.D., Potthast, A., Schwartz, E.L., and Fischl, B. (2008). Accurate prediction of V1 location from cortical folds in a surface coordinate system. Neuroimage 39, 1585-1599.

Horton, J., and Hedley-Whyte, E.T. (1984). Mapping of cytochrome oxidase patches and ocular dominance columns in human visual cortex. Philosophical Transactions of the Royal Society of London. Series B, Biological Sciences, 255-272.

Horton, J.C., Dagi, L.R., Mccrane, E.P., and De Monasterio, F.M. (1990). Arrangement of ocular dominance columns in human visual cortex. Arch Ophthalmol 108, 1025-1031.

Hubel, D.H., and Wiesel, T.N. (1965). Receptive fields and functional architecture in two nonstriate visual areas (18 and 19) of the cat. Journal of neurophysiology 28, 229-289.

Hubel, D.H., and Wiesel, T.N. (1977). Ferrier lecture: Functional architecture of macaque monkey visual cortex. Proceedings of the Royal Society of London B: Biological Sciences 198, 1-59. 
Jones, S.E., Buchbinder, B.R., and Aharon, I. (2000). Three-dimensional mapping of cortical thickness using Laplace's equation. Human Brain Mapping 11, 12-32. doi: 10.1002/10970193(200009)11:1<12::aid-hbm20>3.0.co;2-k.

Katz, L.C., Gilbert, C.D., and Wiesel, T.N. (1989). Local circuits and ocular dominance columns in monkey striate cortex. The Journal of Neuroscience 9, 1389-1399.

Kemper, V.G., De Martino, F., Tse, D.H., Poser, B.A., Yacoub, E., and Goebel, R. (2015a). T2 weighted high-resolution $\mathrm{fMRI}$ in human visual cortex at 9.4 T using 3D-GRASE. 23nd Annual Meeting of ISMRM 23, 1944.

Kemper, V.G., De Martino, F., Vu, A.T., Poser, B.A., Feinberg, D.A., Goebel, R., and Yacoub, E. (2015b). Sub-Millimeter T2 Weighted fMRI at 7 T: Comparison of 3D-GRASE and 2D SE-EPI. Frontiers in Neuroscience 9. doi: 10.3389/fnins.2015.00163.

Kemper, V.G., De Martino, F., Yacoub, E., and Goebel, R. (2015c). Variable flip angle 3D-GRASE for high resolution fMRI at 7 tesla. Magn Reson Med. doi: 10.1002/mrm.25979.

Lee, J., Hirano, Y., Fukunaga, M., Silva, A.C., and Duyn, J.H. (2010). On the contribution of deoxy-hemoglobin to MRI gray-white matter phase contrast at high field. Neuroimage 49, 193-198.

Levay, S., Connolly, M., Houde, J., and Van Essen, D. (1985). The complete pattern of ocular dominance stripes in the striate cortex and visual field of the macaque monkey. The Journal of neuroscience 5, 486501.

Liang, X., Connelly, A., Tournier, J.D., and Calamante, F. (2014). A variable flip angle-based method for reducing blurring in 3D GRASE ASL. Phys Med Biol 59, 5559-5573. doi: 10.1088/00319155/59/18/5559.

Lutti, A., Dick, F., Sereno, M.I., and Weiskopf, N. (2014). Using highresolution quantitative mapping of $\mathrm{R} 1$ as an index of cortical myelination. Neuroimage 93 Pt 2, 176-188. doi: 10.1016/j.neuroimage.2013.06.005.

Malach, R., Amir, Y., Harel, M., and Grinvald, A. (1993). Relationship between intrinsic connections and functional architecture revealed by optical imaging and in vivo targeted biocytin injections in primate striate cortex. Proceedings of the National Academy of Sciences 90, 10469-10473.

Marques, J.P., Kober, T., Krueger, G., Van Der Zwaag, W., Van De Moortele, P.F., and Gruetter, R. (2010). MP2RAGE, a self bias-field corrected sequence for improved segmentation and T1-mapping at high field. 
Neuroimage 49, 1271-1281. doi: 10.1016/j.neuroimage.2009.10.002.

Matsubara, J., Cynader, M., and Swindale, N. (1987). Anatomical properties and physiological correlates of the intrinsic connections in cat area 18. The Journal of neuroscience 7, 1428-1446.

Menon, R.S., Ogawa, S., Strupp, J.P., and Ugurbil, K. (1997). Ocular dominance in human V1 demonstrated by functional magnetic resonance imaging. Journal of Neurophysiology 77, 2780-2787.

Nehrke, K., and Börnert, P. (2012). DREAM - a novel approach for robust, ultrafast, multislice B1 mapping. Magnetic Resonance in Medicine 68, 1517-1526.

Nieuwenhuys, R. (2013). "The myeloarchitectonic studies on the human cerebral cortex of the Vogt-Vogt school, and their significance for the interpretation of functional neuroimaging data," in Microstructural Parcellation of the Human Cerebral Cortex. Springer), 55-125.

Ogawa, S., Lee, T.M., Kay, A.R., and Tank, D.W. (1990). Brain magnetic resonance imaging with contrast dependent on blood oxygenation. Proc Natl Acad Sci U S A 87, 9868-9872.

Pohmann, R., Speck, O., and Scheffler, K. (2015). Signal-to-noise ratio and MR tissue parameters in human brain imaging at 3, 7, and 9.4 tesla using current receive coil arrays. Magn Reson Med. doi: $10.1002 / \mathrm{mrm} .25677$.

Ramón Y Cajal, S. (1899). Comparative study of the sensory areas of the human cortex.

Renvall, V., Witzel, T., Wald, L.L., and Polimeni, J.R. (2014). Fast variable inversion-recovery time EPI for anatomical reference and quantitative T1 mapping. 22nd Annual Meeting of ISMRM 22, 4282.

Sánchez-Panchuelo, R.M., Francis, S.T., Schluppeck, D., and Bowtell, R.W. (2012). Correspondence of human visual areas identified using functional and anatomical MRI in vivo at $7 \mathrm{~T}$. Journal of Magnetic Resonance Imaging 35, 287-299.

Scheffler, K., and Ehses, P. (2015). High-resolution mapping of neuronal activation with balanced SSFP at 9.4 tesla. Magnetic resonance in medicine.

Schira, M.M., Tyler, C.W., Breakspear, M., and Spehar, B. (2009). The foveal confluence in human visual cortex. The Journal of Neuroscience 29, 9050-9058.

Senden, M., Reithler, J., Gijsen, S., and Goebel, R. (2014). Evaluating population receptive field estimation frameworks in terms of robustness and reproducibility. PLoS One 9, e114054. doi: 10.1371/journal.pone.0114054. 
Shoham, D., Hübener, M., Schulze, S., Grinvald, A., and Bonhoeffer, T. (1997). Spatio-temporal frequency domains and their relation to cytochrome oxidase staining in cat visual cortex. Nature 385, 529533.

Silver, M.A., and Kastner, S. (2009). Topographic maps in human frontal and parietal cortex. Trends Cogn Sci 13, 488-495. doi: 10.1016/j.tics.2009.08.005.

Ts'o, D.Y., Frostig, R.D., Lieke, E.E., and Grinvald, A. (1990). Functional organization of primate visual cortex revealed by high resolution optical imaging. Science 249, 417-420.

Tse, D.H., Poole, M.S., Magill, A.W., Felder, J., Brenner, D., and Jon Shah, N. (2014). Encoding methods for B1(+) mapping in parallel transmit systems at ultra high field. J Magn Reson 245, 125-132. doi: 10.1016/j.jmr.2014.06.006.

Uludag, K., Muller-Bierl, B., and Ugurbil, K. (2009). An integrative model for neuronal activity-induced signal changes for gradient and spin echo functional imaging. Neuroimage 48, 150-165. doi: 10.1016/j.neuroimage.2009.05.051.

Van De Moortele, P.F., Auerbach, E.J., Olman, C., Yacoub, E., Ugurbil, K., and Moeller, S. (2009). T1 weighted brain images at 7 Tesla unbiased for Proton Density, $\mathrm{T} 2 *$ contrast and RF coil receive B1 sensitivity with simultaneous vessel visualization. Neuroimage 46, 432-446. doi: 10.1016/j.neuroimage.2009.02.009.

Vogt, C., and Vogt, O. (1919). Allgemeine ergebnisse unserer hirnforschung. JA Barth.

Waehnert, M.D., Dinse, J., Weiss, M., Streicher, M.N., Waehnert, P., Geyer, S., Turner, R., and Bazin, P.L. (2014). Anatomically motivated modeling of cortical laminae. Neuroimage 93 Pt 2, 210-220. doi: 10.1016/j.neuroimage.2013.03.078.

Wandell, B.A., and Winawer, J. (2011). Imaging retinotopic maps in the human brain. Vision Res 51, 718-737. doi: 10.1016/j.visres.2010.08.004.

Wilms, M., Eickhoff, S.B., Homke, L., Rottschy, C., Kujovic, M., Amunts, K., and Fink, G.R. (2010). Comparison of functional and cytoarchitectonic maps of human visual areas V1, V2, V3d, V3v, and V4(v). Neuroimage 49, 1171-1179. doi: 10.1016/j.neuroimage.2009.09.063.

Winawer, J., Horiguchi, H., Sayres, R.A., Amano, K., and Wandell, B.A. (2010). Mapping hV4 and ventral occipital cortex: the venous eclipse. Journal of Vision 10, 1.

Wohlschläger, A.M., Specht, K., Lie, C., Mohlberg, H., Wohlschläger, A., Bente, K., Pietrzyk, U., Stöcker, T., Zilles, K., and Amunts, K. (2005). 
Linking retinotopic $\mathrm{fMRI}$ mapping and anatomical probability maps of human occipital areas V1 and V2. Neuroimage 26, 73-82.

Yacoub, E., Harel, N., and Ugurbil, K. (2008). High-field fMRI unveils orientation columns in humans. Proc Natl Acad Sci U S A 105, 10607-10612. doi: 10.1073/pnas.0804110105.

Yacoub, E., Shmuel, A., Logothetis, N., and Ugurbil, K. (2007). Robust detection of ocular dominance columns in humans using Hahn Spin Echo BOLD functional MRI at 7 Tesla. Neuroimage 37, 1161-1177. doi: 10.1016/j.neuroimage.2007.05.020.

Yacoub, E., Van De Moortele, P.F., Shmuel, A., and Ugurbil, K. (2005). Signal and noise characteristics of Hahn SE and GE BOLD fMRI at $7 \mathrm{~T}$ in humans. Neuroimage 24, 738-750. doi: 10.1016/j.neuroimage.2004.09.002.

Yoshioka, T., Blasdel, G.G., Levitt, J.B., and Lund, J.S. (1996). Relation between patterns of intrinsic lateral connectivity, ocular dominance, and cytochrome oxidase-reactive regions in macaque monkey striate cortex. Cerebral Cortex 6, 297-310.

Zaitsev, M., Dold, C., Sakas, G., Hennig, J., and Speck, O. (2006). Magnetic resonance imaging of freely moving objects: prospective real-time motion correction using an external optical motion tracking system. Neuroimage 31, 1038-1050.

Zimmermann, J., Goebel, R., De Martino, F., Van De Moortele, P.F., Feinberg, D., Adriany, G., Chaimow, D., Shmuel, A., Ugurbil, K., and Yacoub, E. (2011). Mapping the organization of axis of motion selective features in human area MT using high-field fMRI. PLOS One 6, e28716. doi: 10.1371/journal.pone.0028716. 
Chapter 5

Summary and discussion 
MRI acquisitions present an abundance of trade-offs and compromises between competing factors for optimal results, and fMRI techniques constitute no exception to this rule. The choice of the optimal functional contrast mechanism $\left(T_{2}{ }^{*}\right.$ weighted $B O L D, T_{2}$ weighted $\left.B O L D, C B V, C B F\right)$ for a given application is fundamental and by no means trivial (but often dictated by practical considerations). After opting for a basic experimental design and imaging sequence type, the imaging parameters need to be set to satisfy the needs of a given application (e.g. high spatial resolution mapping, encoding/decoding of brain states, whole-brain resting state) for subject safety (SAR and peripheral nerve stimulation limits), spatial resolution, temporal resolution, spatial coverage, distortions, short experiment duration, functional specificity, and functional sensitivity all at the same time (see Figure 5-1). All current high resolution fMRI approaches have their pros and cons, and the fact that a remedy for a typical downside of a given approach in special cases might be at hand makes things only more confusing. The methodological contributions of this thesis extend the knowledge in the field of $T_{2}$ weighted ultra-high field $\mathrm{fMRI}$, exploring possibilities and limitations of the acquisition and analysis techniques for this contrast mechanism. In this chapter, we will summarize our empirical findings and then explore two aspects of particular relevance to high resolution fMRI further: Avoidance of macrovascular signals and precise coregistration with anatomical reference images. Finally, we will give an account of personally presumed future developments and open challenges.

\section{Summary}

In Chapter 2 we have shown how two $T_{2}$ weighted $\mathrm{fMRI}$ sequences, 3DGRASE and 2D SE-EPI compare at 7T in a submillimeter resolution experiment. 3D-GRASE yielded superior specificity and sensitivity over SEEPI with large field of view. Spatial coverage and image blurring were better in SE-EPI. The superior sensitivity was reflected in more robust activations in a visual task. Besides the SNR-beneficial 3D acquisition in 3DGRASE, the 2D multi-slice slice profiles were identified to hamper the tSNR in 2D SE-EPI. In high resolution cortical depth profiles, 2D SE-EPI revealed an increase of BOLD signal changes towards superficial layers, resembling previously observed GE-EPI profiles but with a smaller slope. The fact that in deep cortical gray matter the signal changes were similar suggested that principally, similar contrast mechanisms were at work, however, the 
increase towards the surface was in line with a higher $T_{2}{ }^{*}$ contrast contamination in 2D SE-EPI. Moreover, we demonstrated that the difference in superficial layers was not a mere consequence of blurring by an analysis of smoothed 2D SE-EPI data and of the angle between the cortex normal and the 3D-GRASE slabs in two orthogonal orientations. We concluded that 3D-GRASE is the preferred method for the investigation of fine functional structures, however acknowledging the limited spatial coverage and strong slice blurring.

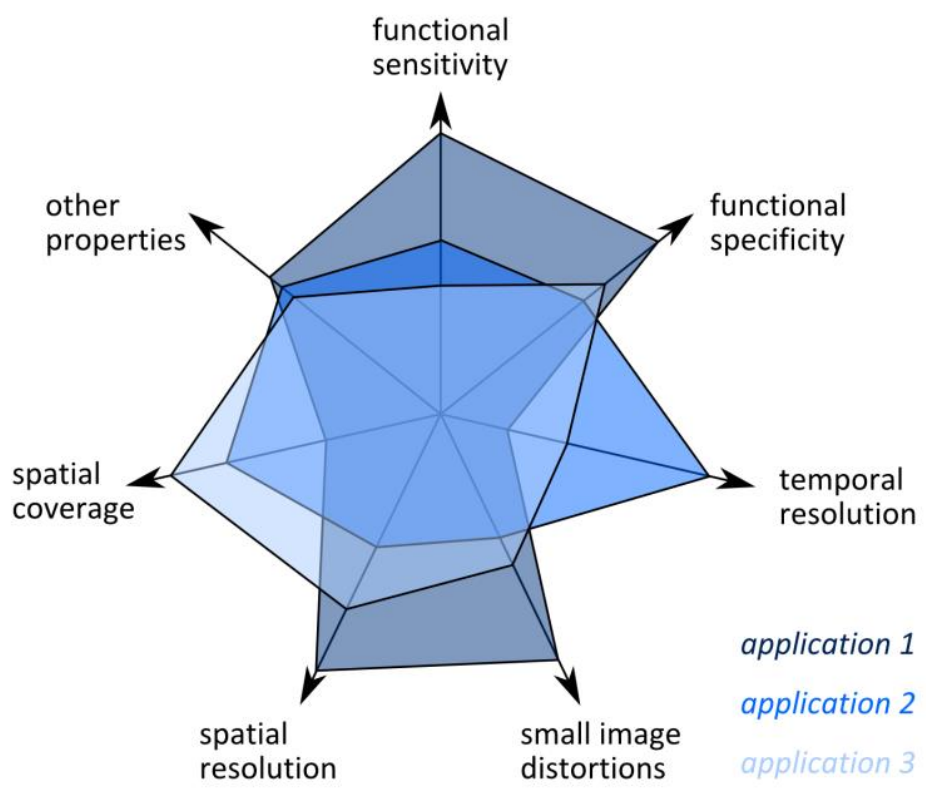

Figure 5-1: Example profiles of required characteristics of fMRI applications. Multiple properties (dimensions) are displayed in a polar diagram.

In Chapter 3, we addressed these issues of 3D-GRASE by introducing a variable flip angle refocusing approach for 3D-GRASE based on extended phase graph theory. This made a simultaneous improvement of coverage and image blurring possible. The VFA approach grants more flexibility in the use of 3D-GRASE to find balance points of the acquisition properties mentioned above which suit the application best. Specifically, coverage and image blurring could be significantly improved compared to the standard 
$180^{\circ}$ refocusing approach at little cost in functional sensitivity. Moreover, SAR becomes significantly less of a limitation using the smaller refocusing angles. Despite the fact that TSNR was reduced in the VFA approach, functional sensitivity was partly maintained due to a small observed increase in functional contrast in the functional experiment at $7 \mathrm{~T}$. This is mediated by a shift of signal towards the longer TE pathways in stimulated echo pathways. The agreement of simulations and experimental data was first validated in phantom measurements. In-vivo, we were able to obtain blurring similar to that observed in 2D SE-EPI in chapter 1 . The VFA approach was more susceptible to $\mathrm{B}_{1}{ }^{+}$inhomogeneity yielding reduced tSNR and functional sensitivity when the transmission was insufficient.

In Chapter 4, we explored the possibilities of human anatomical and functional MRI at 9.4 T further. With a specialized RF coil with spatially limited transmit- and receive profiles, we were able to conduct the experiments well within the SAR limits using a non-subject-specific $B_{1}$ shim with high RF efficiency and sufficient homogeneity. Good anatomical contrast was observed in $T_{1}$ weighted MPRAGE acquisitions adapted to the relaxation times at $9.4 \mathrm{~T}$. Further, high resolution $(0.35 \mathrm{~mm}$ isotropic) $\mathrm{T}_{2}{ }^{*}$ weighted data yielded sufficient information to observe clear differences in cortical depth dependent profiles between visual areas V1 and V2 associated with the co-localization of iron with myelin in the Stria of Gennari. A routine population receptive field experiment yielded excellent results affirming the expected SNR advantage of ultra-high field beyond $7 \mathrm{~T}$. By the means of the VFA approach, we were able to apply 3D-GRASE also at $9.4 \mathrm{~T}$ for a functional mapping experiment at $0.7 \mathrm{~mm}$ isotropic nominal resolution. These measurements showed significant differences in ocular dominance between retinotopically defined V1 and V2. Further, our preliminary results revealed a patchy organization of ocular dominance in V1 of one of the two volunteers. This organization was found to be consistent across cortical depth.

\section{Avoiding macrovascular signals in $\mathrm{T}_{2}{ }^{*}$ and $\mathrm{T}_{2}$ weighted BOLD data}

Functional specificity is the key advantage of high field $T_{2}$ weighted BOLD over $\mathrm{T}_{2}{ }^{*}$ weighted BOLD fMRI, which has previously been demonstrated extensively (in methodological and application studies, see Introduction for details). However, in most imaging aspects aside of functional specificity, GE-EPI sets high standards, particularly functional sensitivity. Could it be 
that the overall higher sensitivity over-compensates the lower specificity, or is the higher specificity of $T_{2}$ more beneficial? A few aspects of this question will be outlined in the following section.

To begin with, taking a purely quantitative stand-point, it must be noted that the expected signal changes from micro-vasculature are not fundamentally different between $\mathrm{T}_{2}$ and $\mathrm{T}_{2}{ }^{*}$ weighted BOLD imaging. For both, extravascular signal changes are simulated to be between 1 and $2 \%$ at ultra-high field (Uludag et al., 2009). The intravascular component is negligible. Empirically, Yacoub et al. (2005) reported the $\mathrm{T}_{2}{ }^{*}$ signal to be on average $1.3 \pm 0.1$ times higher than the $T_{2}$ signal in gray matter at high spatial resolution at $7 \mathrm{~T}$. Stronger differences in sensitivity must be the result of lower tSNR, and in fact, lower tSNR is reported in comparative studies when different TEs and relaxation times are taken into consideration (Harmer et al., 2012; Boyacioglu et al., 2014). This reduction in tSNR in $T_{2}$ weighted data could be a consequence of poor slice profiles and saturation effects, as discussed in Chapter 2 of this thesis. The spinecho signal suffers more strongly from imperfect slice profiles because of the combined $\sin (\pi \cdot f / 2)^{3}$-dependence on the RF scaling factor $f$ of excitation and refocusing pulse, as compared to $\sin \left(\alpha_{E} \cdot f\right)$ in gradient-echo, where $\alpha_{E}$ is the Ernst angle. Technical imperfections could thus explain a significant part of the difference in sensitivity - irrespective of potential differences in physiological noise contributions. The discrepancy of results between Yacoub et al. (2005) and Triantafyllou et al. (2009) with regard to physiological noise in $T_{2}$ weighted data is however noted: While the former reported a constant ratio of physiological to thermal noise with increasing voxel size, the latter reported a similar increase as in $\mathrm{T}_{2}{ }^{*}$ weighted data. Panchuelo et al. (2015) used retinotopic response patterns to demonstrate that the CNRs of $\mathrm{T}_{2}$ and $\mathrm{T}_{2}{ }^{*}$ weighted BOLD imaging converge when the cortical distance between neighboring responses is reduced to $1 \mathrm{~mm}$. Unfortunately, the study did not investigate smaller distances, which would reveal whether $T_{2}$ weighted BOLD excels at higher resolution.

One argument for the utility of a sensitive but unspecific approach is that non-specific signals should cancel each other out in a differential fMRI approach. However, this is only true for balanced conditions, in which the unspecific signals are zero-mean (Polimeni et al., 2010). Further, unrelated signal changes impede the quality of activation estimates (e.g. predictors in a GLM are less efficient). As a remedy, it has been suggested to mask image 
positions which are known to contain large vessels, particularly large pial vessels (Barth and Norris, 2007; Koopmans et al., 2010) in $\mathrm{T}_{2}{ }^{*}$ weighted data, or, in a more drastic form to ignore all data except from middle cortical depth altogether (Goncalves et al., 2015). This approach exploits that large vessels appear dark and tend to have high dynamic signal changes (Olman et al., 2007), or the position of large vessels is obtained from other, co-registered scans. This approach is viable under two conditions: 1) The spatial resolution is sufficiently high to rule out partial volume effects from surface vessels in middle layers (and below). 2) It can be afforded to discard the tissue affected by the vessels, which may be near or far away. This can be the case, e.g. when the aim of the study is to find evidence for the presence of information in a given region or cortical layer using multi-variate pattern analysis (Haxby et al., 2001; Smith and Muckli, 2010; Muckli et al., 2015), or when data can be averaged across a larger cortical region of interest. However, if the intention is to map a given region, the approach does not present a solution. In particular, ignoring signals from superficial layers preempts the possibility to draw any conclusions about columnar (that is vertical, depth-dependent) organization or to target feedback connections which terminate in superficial layers (Muckli et al., 2015). At best, the knowledge about the presence of macrovasculature may give a good estimate of the reliability of the functional data at a given location, as has also been demonstrated at lower resolution (Winawer et al., 2010). Nonetheless, Muckli et al. (2015) were able to decode, which partly occluded visual scene subjects viewed, from superficial cortical layers using GE-EPI in a retinotopic quadrant, which did not receive any visual input. The decoding accuracy was improved after removing non-specific venous signals. $T_{2}$ weighted 3DGRASE measurements in the same subjects yielded lower decoding accuracies. This underscores the potential of $\mathrm{T}_{2}{ }^{*}$ weighted BOLD for decoding applications, even in superficial layers.

Recent experiments (Moerel et al., under review) show that at high resolution the fidelity of gray matter voxels is inversely correlated with the distance to the nearest (medium-sized to large) blood vessel (both tangentially or radially to the cortex) as detected by a time of flight angiography. This is also in line with the observation that physiological noise affects superficial cortical layers stronger than deeper layers (Polimeni et al., 2015) and the concomitantly reduced spatial specificity in 
superficial layers (Polimeni et al., 2010). To what extent responses in middle and deep layers are affected by pial vein reactions is not clear yet. When critically assessing the successful early reports of ODCs and orientation columns using thick tangential slices (Menon et al., 1997; Cheng et al., 2001; Yacoub et al., 2007; Yacoub et al., 2008) it must be stated that the columnarity was informed from knowledge from invasive animal research and post-mortem human data. Which cortical depths (or laminae) contributed to the collapsed maps is unknown, and without the similarity to primate cortical organization, strictly speaking one could only draw the very limited conclusion that tuning e.g. for ocular preference is not opposite at different cortical depths and hence does not cancel out. Importantly, such inference from animal literature is not given for higher cognitive function, where the non-invasiveness of $\mathrm{fMRI}$ truly unfolds its power. Whether sampling only deep layers using GE-EPI would yield spatial maps more similar to those obtained using more specific SE-EPI is unknown, however, experimental findings showed that the specificity of GE-EPI is slightly lower than that of 3D-GRASE at deep and middle cortical depth, and biases may be introduced in GE-EPI independent of cortical depth (De Martino et al., 2013). This may be either due to long-range effects of large pial vessels, or due to the contribution of intracortical veins, which drain a territory of several square millimeters in deep layers (types V4 and V5), and even transport venous blood from the white matter underneath (type V5) across the cortex (Duvernoy et al., 1981).

A relevant controversy for this context was initiated when it was shown that the orientation of visually presented grating stimuli could be decoded from responses in early visual cortex using multivariate pattern analysis (Kamitani and Tong, 2005), although orientation columns are much smaller than the used low imaging resolution in the GE-EPI experiment. The hypothesis was that the origin of the differential signals were slight sampling biases of orientation columns, but it was countered by showing that a broad-scaled organization could explain the high decoding accuracy (Freeman et al., 2011). Similarly, at higher resolution, GE-EPI might provide enough overall sensitivity to provide information about the brain state, however, resulting spatial maps must be treated very carefully because of the functional point-spread. This was demonstrated by Shmuel et al. (2010) reanalyzing the GE-EPI ODC data from Yacoub et al. (2007). Test-retest stability is an important indicator for the reliability of obtained maps. 
Mixed results with GE-EPI might point at insufficient functional resolution for the investigated structure (Yacoub et al., 2007). As a consequence, more recent studies investigating fine-grained structures (Zimmermann et al., 2011; Goncalves et al., 2015) have adapted a modeling approach similar to as by Chaimow et al. (2011) to examine the possibility of picking up biases rather than real topographic representations of unknown spatial scale. In this modelling approach, a putative columnar organization of various sizes and other properties is modelled, and the effects of BOLD point-spread and image sampling are considered.

Finally, the question has to be addressed carefully to what extent larger vessels go unnoticed and are included in the analysis. Draining venules in the cortex have a diameter of 20 to $150 \mu \mathrm{m}$ (Duvernoy et al., 1981; Polimeni et al., 2010). In this regime, there are already distinct differences in the sensitivity of $T_{2}$ and $T_{2}{ }^{*}$ weighted BOLD due to the static broadening effect (Uludag et al., 2005), however, despite the signal reduction also surrounding the vessel in $\mathrm{T}_{2}{ }^{*}$ weighted imaging, these may not be seen even at high spatial resolution fMRI. Hence, fine-grained laminar functional organization might still be clouded by the non-differential response stemming from these. Finally, the notion that cortical vascularization is at least linked to the functional architecture which it supports, has been proposed (Gardner, 2010). If future research proves this hypothesis right to a finer scale than currently known (Harrison et al., 2002), researchers may worry a little less about sensitivity to medium sized blood vessels in the cortex.

\section{Co-registration with structural images}

The study reported in Chapter 4 pushes the spatial resolution both in functional and anatomical acquisitions. In this high resolution domain, the co-registration with structural images with strong anatomical contrast, particularly WM/GM contrast has proven useful, but distortions of high spatial resolution functional data impose difficulties. The reduced distortions of short EPI trains as in zoomed 3D-GRASE have been mentioned at the appropriate places in the previous chapters. However, when larger areas require a precise alignment, the residual distortions will again impede the analysis. Sequences which are virtually free of distortions may present a solution. Promising candidates, which also provide a contrast similar to $\mathrm{T}_{2}$ weighting have recently been presented (Auerbach et 
al., 2002; Ehses et al., 2013; Goa et al., 2013; Hua et al., 2013b; Goa et al., 2014; Scheffler and Ehses, 2015). It should, however, be noted that even these sequences have distortions because of gradient nonlinearities and susceptibility induced $B_{0}$ inhomogeneity. When they are acquired with a different slice prescription than the reference images, residual distortions still hamper the alignment. Moreover, the structural reference scans are also slightly distorted (although the PSF has been improved by the application of variable flip angle echo trains and improved acquisition order (Mugler et al., 1992; Deichmann et al., 2000; Stöcker and Shah, 2006) and accelerated imaging (Van der Kouwe et al., 2014)). Nonetheless, the coregistration might not fulfill the strict requirements of high-resolution $\mathrm{fMRI}$ so that segmentation on the basis of the functional data itself (e.g. averaged across time for better SNR) may be the only alternative. This is possible when the functional data has sufficient anatomical contrast. If this is not the case, the closest proxy to this is an anatomical acquisition with the same distortions and same slice prescription. This approach has recently been revived for $T_{1}$ weighted inversion recovery EPI data (Clare and Jezzard, 2001; Renvall et al., 2014), and may hold great potential for high resolution $\mathrm{fMRI}$.

\section{Challenges and future directions}

The number of research centers operating high field MRI scanners has increased steadily in recent years. Soon, commercial $7 \mathrm{~T}$ systems with clinical CE and FDA approval will be on the market. The growing user group will hopefully nourish further methodological advances in the field of high resolution $\mathrm{fMRI}$ and enable exciting applications in the human brain. Experimental set-ups are exploring double digit field strengths (10.5 T operational at CMRR, Minnesota), and chances are that technical developments necessitated by higher field also translate to improved imaging at lower field strength, as it was the case between $7 \mathrm{~T}$ and $3 \mathrm{~T}$. Time and again, innovations have revolutionized the way MRI is performed - compressed sensing (Lustig et al., 2007) and MR fingerprinting (Ma et al., 2013) to name just two recent examples. If one ventures a prediction, one can only start out from the currently conceivable techniques. In the following, we will therefore try to identify areas of development in the domain of high resolution $\mathrm{fMRI}$ acquisition. 


\section{Image acquisition and reconstruction}

The importance of the imaging PSF has been stressed in this thesis. The blurring effect of signal decay throughout an echo train in EPI, 3D-GRASE, or TSE has been well characterized in the literature, but an additional blurring effect occurs from inconsistencies of the phase along the direction in question. Characterizing and correcting such phase inconsistencies caused by subject motion or other physiological contributions would further improve the PSF of 3D-GRASE. FMRI sequences with better point spread have been mentioned in the above section. They are promising for precise measurements; however, for serious application in submillimeter resolution fMRI, sufficiently high functional sensitivity remains to be demonstrated as of now. The lower temporal efficiency than in echo-train sequences impedes their sensitivity and typically requires moderately high acceleration factors for acceptable temporal resolution (Hua et al., 2013b; Scheffler and Ehses, 2015). Application of reduced field of view imaging to T2prep-GRE or SSFP approaches might overcome these hindrances.

Such reduced field of view techniques can be realized using parallel transmission (pTX) (Katscher et al., 2003; Zhu, 2004), which will see wider application in high field $\mathrm{fMRI}$, especially in $\mathrm{T}_{2}$ weighted BOLD imaging. Zoomed imaging approaches can benefit from pTX, whether in the form of more efficient outer-volume suppression or of 2D or even 3D spatially selective pulses. Tightly linked to improved RF coil designs, PTX has great potential to improve the image acquisition because of the crucial dependence on the precision of RF pulses in spin-echo sequences (particularly echo-train sequences such as 3D-GRASE), and the currently limiting factor of power deposition (SAR). PTX pulse design can accommodate both aspects (Wu et al., 2013). This way, SNR and spatial coverage in multi-slice imaging will be improved. Further, pTX will improve simultaneous multi-slice acquisitions (Poser et al., 2014), which have undergone substantial developments themselves to set new standards in imaging speed (Setsompop et al., 2013; Ugurbil et al., 2013; Xu et al., 2013).

Concomitantly, the supervision of safe operation with regard to power deposition and tissue heating is in need of future improvement at two levels. Fundamentally, possible divergence of SAR and tissue heating currently require additional safety margins (Shrivastava et al., 2008 and 
references therein). Improvements in this aspect would be highly desirable, e.g. direct, precise, and accurate temperature measurements. However, given the slow pace in this domain thus far, this may not be within reach soon. Practically, the current supervision by virtual observation points (Eichfelder and Gebhardt, 2011) slows down the system operation hindering the effective use of multiple different PTX pulses altogether, and improved computations would facilitate the application.

\section{Comparison of different acquisition approaches}

Competing characteristics of the image acquisition such as spatial coverage, functional specificity, and functional sensitivity have been mentioned in the beginning of this discussion. In the multi-dimensional space spanned by these characteristics, different applications require very different profiles (see Figure 5-1). Methodological improvements help to extent the accessible volume in this space. Ideally, one axis, e.g. spatial resolution, could be extended thanks to an innovation without compromising another one, but realistically, most if not all novel approaches have their drawbacks (at least in the moment of their introduction). Over time, a large diversity of different functional contrasts and acquisition methods has developed and to this end, no overall optimal strategy for all high resolution $\mathrm{FMRI}$ studies in humans has been found. 2D GE-EPI is the de facto standard, and comparisons between divergent "branches" of methods are scarce. More comparisons would be desirable to obtain a more coherent, informed picture of the pros and cons of different approaches. Research collaborations between centers to fuse the expertise in the various approaches seem to be a necessary requirement for the successful and neutral assessment of different techniques. Establishment of a reference experiment with a known "gold standard" would be desirable. Such a gold standard would have to be based on wellknown examples of neuronal activity.

The assessment of different approaches should go hand in hand with an advance in theoretical modeling at multiple levels. To this end, many experimental design choices are still led by intuition rather than solid simulations. For example, when intending to map a columnar structure, to what extend are partial Fourier and/or accelerated imaging acquisitions beneficial given the difference in SNR, distortions, and image blurring? Given the profile of BOLD activations in k-space (which differs from that of 
static images), is tSNR the optimal target property to be maximized or could the acquisition be tailored to capture BOLD patterns best? Can the slightly higher sensitivity to capillaries of GE-EPI afford its lower specificity compared to $T_{2}$ weighted BOLD, or should researchers invest more time in techniques with even lower sensitivity and higher specificity such as perfusion or CBV based contrasts? Given the spatio-temporal BOLD PSF, would joint changes in stimulus presentation and data acquisition produce better results? It is evident that the answers to such questions differ depending on the specific application, e.g. mapping versus decoding. However, answers could only be found in synergy of simulations and empirical studies such that eventually, critical balance point values of diverse imaging characteristics might be found. 


\section{References}

Auerbach, E.J., Heberlein, K., and Hu, X. (2002). High-resolution T2 fMRI at high magnetic fields using PSIF. Proceedings of the 10th Annual Meeting of ISMRM, Honolulu, Hawaii, 2345.

Barth, M., and Norris, D.G. (2007). Very high-resolution three-dimensional functional MRI of the human visual cortex with elimination of large venous vessels. NMR Biomed 20, 477-484. doi: 10.1002/nbm.1158.

Boyacioglu, R., Schulz, J., Muller, N.C., Koopmans, P.J., Barth, M., and Norris, D.G. (2014). Whole brain, high resolution multiband spinecho EPI fMRI at 7T: A comparison with gradient-echo EPI using a color-word Stroop task. Neuroimage 97, 142-150. doi: 10.1016/j.neuroimage.2014.04.011.

Chaimow, D., Yacoub, E., Ugurbil, K., and Shmuel, A. (2011). Modeling and analysis of mechanisms underlying fMRI-based decoding of information conveyed in cortical columns. Neuroimage 56, 627642. doi: 10.1016/j.neuroimage.2010.09.037.

Cheng, K., Waggoner, R.A., and Tanaka, K. (2001). Human ocular dominance columns as revealed by high-field functional magnetic resonance imaging. Neuron 32, 359-374. doi: 10.1016/s08966273(01)00477-9.

Clare, S., and Jezzard, P. (2001). Rapid T(1) mapping using multislice echo planar imaging. Magn Reson Med 45, 630-634.

De Martino, F., Zimmermann, J., Muckli, L., Ugurbil, K., Yacoub, E., and Goebel, R. (2013). Cortical depth dependent functional responses in humans at 7T: improved specificity with 3D GRASE. PLoS One 8, e60514. doi: 10.1371/journal.pone.0060514.

Deichmann, R., Good, C.D., Josephs, O., Ashburner, J., and Turner, R. (2000). Optimization of 3-D MP-RAGE sequences for structural brain imaging. Neuroimage 12, 112-127. doi: 10.1006/nimg.2000.0601.

Duvernoy, H.M., Delon, S., and Vannson, J. (1981). Cortical blood vessels of the human brain. Brain research bulletin 7, 519-579.

Ehses, P., Budde, J., Shajan, G., and Scheffler, K. (2013). T2-weighted BOLD fMRI at 9.4 T using a S2-SSFP-EPI sequence. 21st Annual Meeting of ISMRM 21, 0414.

Eichfelder, G., and Gebhardt, M. (2011). Local specific absorption rate control for parallel transmission by virtual observation points. Magnetic Resonance in Medicine 66, 1468-1476.

Freeman, J., Brouwer, G.J., Heeger, D.J., and Merriam, E.P. (2011). Orientation decoding depends on maps, not columns. J Neurosci 31, 4792-4804. doi: 10.1523/JNEUROSCI.5160-10.2011. 
Gardner, J.L. (2010). Is cortical vasculature functionally organized? Neuroimage 49, 1953-1956. doi: 10.1016/j.neuroimage.2009.07.004.

Goa, P.E., Koopmans, P.J., Poser, B.A., Barth, M., and Norris, D.G. (2014). BOLD fMRI signal characteristics of S1- and S2-SSFP at 7 Tesla. Frontiers in Neuroscience 8. doi: 10.3389/fnins.2014.00049.

Goa, P.E., Poser, B.A., and Barth, M. (2013). Modeling and suppression of respiration induced $\mathrm{BO}$-fluctuations in non-balanced steady-state free precession sequences at 7 Tesla. Magnetic Resonance Materials in Physics Biology and Medicine 26, 377-387. doi: 10.1007/s10334-012-0343-6.

Goncalves, N.R., Ban, H., Sanchez-Panchuelo, R.M., Francis, S.T., Schluppeck, D., and Welchman, A.E. (2015). 7 tesla FMRI reveals systematic functional organization for binocular disparity in dorsal visual cortex. J Neurosci 35, 3056-3072. doi: 10.1523/jneurosci.3047-14.2015.

Harmer, J., Sanchez-Panchuelo, R.M., Bowtell, R., and Francis, S.T. (2012). Spatial location and strength of BOLD activation in high-spatialresolution $\mathrm{fMRI}$ of the motor cortex: a comparison of spin echo and gradient echo fMRI at 7 T. NMR Biomed 25, 717-725. doi: 10.1002/nbm.1783.

Harrison, R.V., Harel, N., Panesar, J., and Mount, R.J. (2002). Blood capillary distribution correlates with hemodynamic-based functional imaging in cerebral cortex. Cerebral Cortex 12, 225-233.

Haxby, J.V., Gobbini, M.I., Furey, M.L., Ishai, A., Schouten, J.L., and Pietrini, P. (2001). Distributed and overlapping representations of faces and objects in ventral temporal cortex. Science 293, 2425-2430.

Hua, J., Qin, Q., Van Zijl, P.C., Pekar, J.J., and Jones, C.K. (2013b). Wholebrain three-dimensional T2-weighted BOLD functional magnetic resonance imaging at 7 Tesla. Magn Reson Med. doi: 10.1002/mrm.25055.

Kamitani, Y., and Tong, F. (2005). Decoding the visual and subjective contents of the human brain. Nat Neurosci 8, 679-685. doi: $10.1038 / \mathrm{nn} 1444$.

Katscher, U., Bornert, P., Leussler, C., and Van Den Brink, J.S. (2003). Transmit SENSE. Magn Reson Med 49, 144-150. doi: 10.1002/mrm.10353.

Koopmans, P.J., Barth, M., and Norris, D.G. (2010). Layer-Specific BOLD Activation in Human VI. Human Brain Mapping 31, 1297-1304. doi: 10.1002/hbm.20936. 
Lustig, M., Donoho, D., and Pauly, J.M. (2007). Sparse MRI: The application of compressed sensing for rapid MR imaging. Magn Reson Med 58, 1182-1195. doi: 10.1002/mrm.21391.

Ma, D., Gulani, V., Seiberlich, N., Liu, K., Sunshine, J.L., Duerk, J.L., and Griswold, M.A. (2013). Magnetic resonance fingerprinting. Nature 495, 187-192. doi: 10.1038/nature11971.

Menon, R.S., Ogawa, S., Strupp, J.P., and Ugurbil, K. (1997). Ocular dominance in human V1 demonstrated by functional magnetic resonance imaging. Journal of Neurophysiology 77, 2780-2787.

Muckli, L., De Martino, F., Vizioli, L., Petro, L.S., Smith, F.W., Ugurbil, K., Goebel, R., and Yacoub, E. (2015). Contextual Feedback to Superficial Layers of V1. Current Biology.

Mugler, J.P., 3rd, Epstein, F.H., and Brookeman, J.R. (1992). Shaping the signal response during the approach to steady state in threedimensional magnetization-prepared rapid gradient-echo imaging using variable flip angles. Magn Reson Med 28, 165-185. doi: 10.1002/mrm.1910280202.

Olman, C.A., Inati, S., and Heeger, D.J. (2007). The effect of large veins on spatial localization with GE BOLD at $3 \mathrm{~T}$ : Displacement, not blurring. Neuroimage 34, 1126-1135.

Panchuelo, R.M.S., Schluppeck, D., Harmer, J., Bowtell, R., and Francis, S. (2015). Assessing the Spatial Precision of SE and GE-BOLD Contrast at 7 Tesla. Brain topography 28, 62-65.

Polimeni, J.R., Bianciardi, M., Keil, B., and Wald, L.L. (2015). Cortical depth dependence of physiological fluctuations and whole-brain restingstate functional connectivity at 7T. 23nd Annual Meeting of ISMRM 23, 592.

Polimeni, J.R., Fischl, B., Greve, D.N., and Wald, L.L. (2010). Laminar analysis of 7T BOLD using an imposed spatial activation pattern in human V1. Neuroimage 52, 1334-1346. doi: 10.1016/j.neuroimage.2010.05.005.

Poser, B.A., Anderson, R.J., Guérin, B., Setsompop, K., Deng, W., Mareyam, A., Serano, P., Wald, L.L., and Stenger, V.A. (2014). Simultaneous multislice excitation by parallel transmission. Magnetic Resonance in Medicine 71, 1416-1427.

Renvall, V., Witzel, T., Wald, L.L., and Polimeni, J.R. (2014). Fast variable inversion-recovery time EPI for anatomical reference and quantitative T1 mapping. 22nd Annual Meeting of ISMRM 22, 4282.

Scheffler, K., and Ehses, P. (2015). High-resolution mapping of neuronal activation with balanced SSFP at 9.4 tesla. Magnetic resonance in medicine. 
Setsompop, K., Kimmlingen, R., Eberlein, E., Witzel, T., Cohen-Adad, J., Mcnab, J.A., Keil, B., Tisdall, M.D., Hoecht, P., and Dietz, P. (2013). Pushing the limits of in vivo diffusion MRI for the Human Connectome Project. Neuroimage 80, 220-233.

Shmuel, A., Chaimow, D., Raddatz, G., Ugurbil, K., and Yacoub, E. (2010). Mechanisms underlying decoding at $7 \mathrm{~T}$ : ocular dominance columns, broad structures, and macroscopic blood vessels in V1 convey information on the stimulated eye. Neuroimage 49, 19571964. doi: 10.1016/j.neuroimage.2009.08.040.

Shrivastava, D., Hanson, T., Schlentz, R., Gallaghar, W., Snyder, C., Delabarre, L., Prakash, S., laizzo, P., and Vaughan, J.T. (2008). Radiofrequency heating at 9.4T: in vivo temperature measurement results in swine. Magn Reson Med 59, 73-78. doi: 10.1002/mrm.21425.

Smith, F.W., and Muckli, L. (2010). Nonstimulated early visual areas carry information about surrounding context. Proc Natl Acad Sci U S A 107, 20099-20103. doi: 10.1073/pnas.1000233107.

Stöcker, T., and Shah, N.J. (2006). MP-SAGE: A new MP-RAGE sequence with enhanced SNR and CNR for brain imaging utilizing squarespiral phase encoding and variable flip angles. Magnetic resonance in medicine 56, 824-834.

Triantafyllou, C., Polimeni, J.R., Elschot, M., and Wald, L.L. (2009). Physiological Noise in Gradient Echo and Spin Echo EPI at 3T and 7T. 17th Annual Meeting International Society for Magnetic Resonance in Medicine, 122.

Ugurbil, K., Xu, J., Auerbach, E.J., Moeller, S., Vu, A.T., Duarte-Carvajalino, J.M., Lenglet, C., Wu, X., Schmitter, S., Van De Moortele, P.F., Strupp, J., Sapiro, G., De Martino, F., Wang, D., Harel, N., Garwood, M., Chen, L., Feinberg, D.A., Smith, S.M., Miller, K.L., Sotiropoulos, S.N., Jbabdi, S., Andersson, J.L., Behrens, T.E., Glasser, M.F., Van Essen, D.C., and Yacoub, E. (2013). Pushing spatial and temporal resolution for functional and diffusion $\mathrm{MRI}$ in the Human Connectome Project. Neuroimage 80, 80-104. doi: 10.1016/j.neuroimage.2013.05.012.

Uludag, K., Dubowitz, D.J., and Buxton, R.B. (2005). "Principles of functional imaging of the brain.," in Clinical Magnetic Resonance Imaging (3rd Ed), eds. R.R. Edelmann, J.R. Hesselink \& M.B. Zlatkin. (England).

Uludag, K., Muller-Bierl, B., and Ugurbil, K. (2009). An integrative model for neuronal activity-induced signal changes for gradient and spin echo functional imaging. Neuroimage 48, 150-165. doi: 10.1016/j.neuroimage.2009.05.051. 
Van Der Kouwe, A.J., Tisdall, M.D., Bhat, H., Fischl, B., and Polimeni, J.R. (2014). Multiple Echo and Inversion Time MPRAGE with Inner Loop GRAPPA Acceleration and Prospective Motion Correction for Minimally Distorted Multispectral Brain Morphometry. 22nd Annual Meeting of ISMRM 22, 120.

Winawer, J., Horiguchi, H., Sayres, R.A., Amano, K., and Wandell, B.A. (2010). Mapping hV4 and ventral occipital cortex: the venous eclipse. Journal of Vision 10, 1.

Wu, X., Schmitter, S., Auerbach, E.J., Moeller, S., Uğurbil, K., and De Moortele, V. (2013). Simultaneous multislice multiband parallel radiofrequency excitation with independent slice-specific transmit B1 homogenization. Magnetic Resonance in Medicine 70, 630-638.

Xu, J., Moeller, S., Auerbach, E.J., Strupp, J., Smith, S.M., Feinberg, D.A., Yacoub, E., and Ugurbil, K. (2013). Evaluation of slice accelerations using multiband echo planar imaging at 3 T. Neuroimage 83, 9911001. doi: 10.1016/j.neuroimage.2013.07.055.

Yacoub, E., Harel, N., and Ugurbil, K. (2008). High-field fMRI unveils orientation columns in humans. Proc Natl Acad Sci U S A 105, 10607-10612. doi: 10.1073/pnas.0804110105.

Yacoub, E., Shmuel, A., Logothetis, N., and Ugurbil, K. (2007). Robust detection of ocular dominance columns in humans using Hahn Spin Echo BOLD functional MRI at 7 Tesla. Neuroimage 37, 1161-1177. doi: 10.1016/j.neuroimage.2007.05.020.

Yacoub, E., Van De Moortele, P.F., Shmuel, A., and Ugurbil, K. (2005). Signal and noise characteristics of Hahn SE and GE BOLD fMRI at $7 \mathrm{~T}$ in humans. Neuroimage 24, 738-750. doi: 10.1016/j.neuroimage.2004.09.002.

Zhu, Y. (2004). Parallel excitation with an array of transmit coils. Magnetic Resonance in Medicine 51, 775-784.

Zimmermann, J., Goebel, R., De Martino, F., Van De Moortele, P.F., Feinberg, D., Adriany, G., Chaimow, D., Shmuel, A., Ugurbil, K., and Yacoub, E. (2011). Mapping the organization of axis of motion selective features in human area MT using high-field fMRI. PLoS One 6, e28716. doi: 10.1371/journal.pone.0028716. 


\section{Knowledge Valorization}

According to the "National Valorization Committee", the term knowledge valorization refers to "the process of creating value from knowledge, by making knowledge suitable and/or available for social (and/or economic) use and by making knowledge suitable for translation into competitive products, services, processes and new commercial activities" (Regulations governing the attainment of doctoral degree, UM, App. 4, §23), and the doctoral candidate is requested to write "an approximately five-page addendum" on the topic, which, however, shall not be a part of the dissertation itself.

So, how can the insights of the present thesis be "valorized"? The present thesis is mostly methodological in nature, that is, for the most part it deals with the question of how T2 weighted high resolution BOLD FMRI in visual cortex can be performed better for cognitive neuroscientific applications. Since these applications lie within the field of fundamental natural sciences, the thesis can also be allocated in this domain. Fundamental sciences and the concept of "valorization" are considered diametrically opposed to one another by many, and this conflict has been pointed out in the valorization addendums appended to recent dissertations in the department for cognitive neuroscience (see e.g. R. L. Rademaker, "Internal representations of the brain" (2015); G. Lange, "Psychophysical investigations of perceptual learning and attention" (2016)). However, the results of fundamental research may yield immeasurable value to society in the future via complex and unforeseeable paths. In this light, the answer to the question posed above can impossibly be answered exhaustively. It is clear to see that the degree to which the knowledge is straight-forwardly applicable scales inversely with the "distance" of the specific application to the centers of attention of the present work. In an attempt to illustrate this view, Figure 1 displays a classification of various applications of magnetic resonance imaging. It is needless to say that this binary hierarchical classification is extremely simplified for the purpose of clarity and underestimates the variety and complexity of applications. Some levels may as well be swapped, e.g. the distinctions in species and purposes (otherwise e.g. neuroscientific research on rodents or a diffusion scan for the diagnosis of a brain tumor in a race horse could not be 
accommodated). Further, the branches which do not lead to the BOLD technique are kept very general and are not detailed further.

To the first order, the considerations with regard to "valorization" may be limited to the displayed realm of applications of MRI. The core claim of this chapter will be that the concepts presented in this thesis are applicable in other side branches at all hierarchical levels in figure 1. In other words, any information provided in this thesis may influence decision-making with regard to the techniques for any applications in magnetic resonance imaging. As stated above, the direct applicability to different nodes of the graph then scales inversely with the "distance" (i.e. shortest path in the graph). In the following, we will provide some examples of this.

The most direct application of the findings of this thesis is in other modalities or other functional domains. 3D-GRASE has been used for arterial spin labeling $\mathrm{fMRI}$, and the variable flip angle technique (Chapter 3 ) can improve the data quality in this application, as has also recently been shown by other authors. The thesis discusses advantages and disadvantages of $\mathrm{T} 2$ - and $\mathrm{T} 2 *$-weighted BOLD $\mathrm{fMRI}$ at length (e.g. Chapter 5), and these apply to other functional domains, e.g, any sensory domains, memory, emotions, decision-making etc. just like in the visual domain. The suitability of 2D SE-EPI or 3D-GRASE for these applications may be guided by the findings in Chapter 2 .

The application of the variable flip angle technique proposed for the 3DGRASE imaging pulse sequence in Chapter 3 is most beneficial at long spinecho train duration compared to $\mathrm{T} 2$ and long $\mathrm{T} 1$. These conditions are not only given in brain imaging (functional and structural (anatomical), for which 3D-GRASE is also employed), but may occur in various applications, in various materials and organic, human or non-human tissue types. Examples range from human lung imaging and musculoskeletal imaging to non-hydrogen ("X-Nuclei") imaging in soft tissue, e.g. sodium imaging in knee cartilage, or imaging in solid inorganic materials. The additional degrees of freedom enabled by the variable flip angles may be exploited in larger spatial coverage, reduced image blurring, or higher nominal spatial resolution. Further, the significantly reduced power deposition may enable new applications, especially when SAR is a delicate issue. 

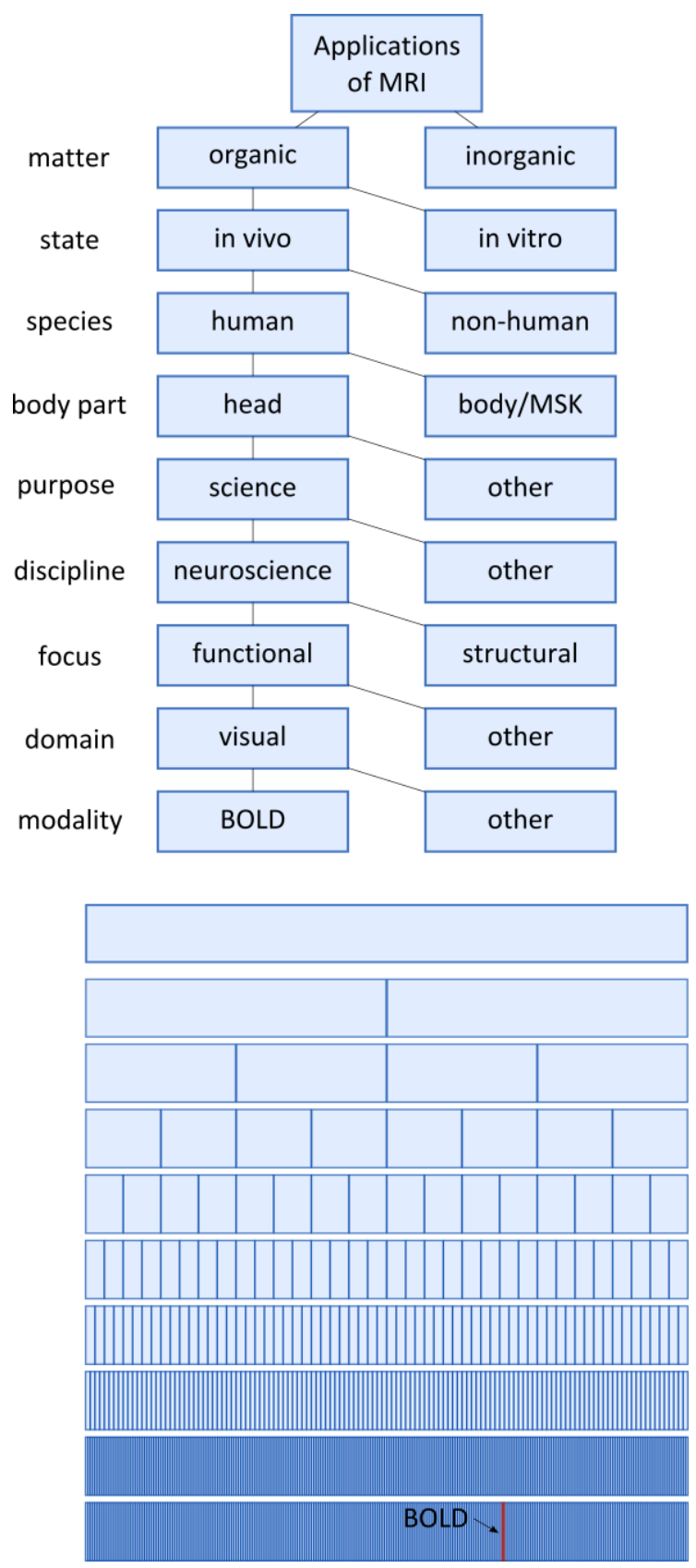

Figure 1: Classification of various applications of magnetic resonance imaging (strongly simplified). Top: Hierarchical binary tree, bottom: Same structure displayed to scale under the assumption of equal binary composition and a random placement of BOLD imaging among other applications. 
The work presented in this thesis relies on ultra-high field imaging. By the methodological advancements in it, it makes ultra-high field imaging more accessible to the various applications mentioned above. The improved signal-to-noise ratio at higher field strength is unquestioned and may be translated to stronger contrast-to-noise ratio, shorter imaging time, and/or higher spatial resolution, all of which may be helpful in clinical routines. These factors would facilitate the diagnosis of known pathologies, but moreover enable diagnosis of previously elusive diseases. Ultimately, these factors will help, e.g. to start patient treatment earlier, and improve its monitoring throughout the process, thereby improving quality of life and life expectancy. Economically, this potential is showcased by the introduction of the first commercially available 7T scanner with CE and FDA approval, the "Terra" manufactured by Siemens Healthcare about fifteen years after the world's first human 7 Tesla MRI scanner was installed at University of Minnesota in 1999. In this context we may also refer to the development of novel RF transmission and receive coil designs. A close link between manufacturers (e.g. Life Services, Minneapolis, MN, USA) and the scientific research institute enabled the customized design for the RF coils used in the studies reported here. Based on this experience (e.g. with regard to the spatial distribution of coil sensitivities and coupling) additional RF coils have been and will be designed for advanced imaging applications, which pushes research forward worldwide. Finally it is worth mentioning that innovations from the ultra-high field domain have previously also translated to improvements at lower field strength, and this process is likely to continue. Given that $1.5 \mathrm{~T}$ and 3.0 $\mathrm{T}$ machines are the mainstay of clinical MRI, methodological improvements at ultra-high field already enhance clinical routine to the benefit of patient care.

Currently, there are merely a handful of operational human MRI systems worldwide which exceed 7T field strength, and our anatomical and functional study at 9.4 T (Chapter 4) is still one of the first of its kind. Whether this even higher field strength will eventually also find its way into clinical routine remains to be seen. However, our study is a door opener in the sense that it demonstrated the feasibility of $9.4 \mathrm{~T}$ scanning in the human brain without the requirement of complicated parallel transmission pulses. This circumstance facilitates the access to 9.4 $\mathrm{T}$ for application in different fields and possibly without the close monitoring by MR physicists after appropriate training of non-expert users. 
It was stated above that the limitation of the "valorization" considerations may be limited to the organization according to Figure 1 to the first order. What was meant by this is that the "valorization" is most direct in this framework. As though this would not already present a (perhaps infinitely) large amount of improved techniques and new possibilities, they themselves open up new paths for improved techniques and new possibilities in other domains - second order - and so on. This cascade describes the sum of marginal utility and leads eventually to an infinite "valorization" potential, as held by all scientific projects. 


\section{Acknowledgements}

I would like to express my gratitude to a number of people who have helped directly or indirectly with the completion of this thesis. Needless to say that it would not have been possible without the contribution of many. My supervisors, Rainer, Essa, and Federico, have done a great job supporting and helping me with every step along the way in the past years. Your dedication to our work is so inspiring. Rainer, your enthusiasm and optimism are truly contagious and were a constant source of motivation for me. Essa, you will always be a role model for me for your calm way of evaluating experimental evidence to then point right to the most critical aspects of them. I knew that a project was good only when it passed your scrutiny. Federico, besides the invaluable feedback and hands-on knowledge you taught me lessons about two important things: passion for what we do, and priorities. Keeping an eye on the overall development of our projects, you also gave me the push I needed time and again to make a decision and move on.

I truly enjoyed the time in the department of cognitive neuroscience, which is characterized by such a positive, constructive spirit of partnership and joint effort. The list of people who contribute to this pleasant atmosphere is almost endless, but I would like to say "thank you" explicitly to the people working in the background for the smooth operation, most of all to Christl, Riny, and Eva. I enjoyed also the work with the Scannexus technicians Lotty, Esther, and the others. Also, I appreciate the companionship of all fellow scientists in Rainer's (ERC) group, in the MR physics group, the CN R\&D team, Brain Innovation, and diverse lunch- and coffee break groups. Please rest assured that our exchange - whether professional, private, or (as often the case) anything in between - is highly valued. This goes particularly for my paranymphs Helen and Thomas, and office mates Sanae, Britta, Jochen, Jessica, and Amaia. You guys make coming to Uni fun. Finally, I would like to state my deep thankfulness to my parents, brothers, and Maren. Life would not be the same without you. 


\section{Curriculum Vitae}

Valentin was born on October 19, 1985, in Coesfeld, Germany. After finishing his secondary education at Cypress Bay High School, Weston, FL., USA, and Schillergymnasium Münster, Germany, in 2005 (Abitur grade 1.3), he studied Psychology and Physics at WWU University of Münster. Valentin graduated from RWTH Aachen University (Diplom-Physiker) in solid-state physics minoring in computer science and biomedical engineering. His diploma thesis under the supervision of Prof.dr. N. Jon Shah and dr. AnaMaria Oros-Peusquens at INM-4 of Jülich Research Centre dealt with "Decomposition of Signal Decay and Recovery of T2* Information in Quantitative MRI". In 2011, he started a PhD project under the supervision of Prof.dr. Rainer Goebel and dr. Federico De Martino of Maastricht University, and Prof.dr. Essa Yacoub of University of Minnesota. After finishing his thesis entitled "Submillimeter BOLD fMRI of human visual cortex" Valentin will continue working in the department for Cognitive Neuroscience in Maastricht under the supervision of dr. De Martino and Prof.dr. Elia Formisano. 


\section{Publication List}

\section{Peer reviewed journal articles}

Kemper, V.G., De Martino, F., Vu, A.T., Poser, B.A., Feinberg, D.A., Goebel, R., and Yacoub, E. (2015). Sub-Millimeter T2 Weighted fMRI at $7 \mathrm{~T}$ : Comparison of 3D-GRASE and 2D SE-EPI. Frontiers in Neuroscience 9. doi: 10.3389/fnins.2015.00163.

Kemper, V.G., De Martino, F., Yacoub, E., and Goebel, R. (2015). Variable flip angle 3D-GRASE for high resolution fMRI at 7 tesla. Magn Reson Med. doi: 10.1002/mrm.25979.

Riecke, L., Peters, J.C., Valente, G., Kemper, V.G., Formisano, E., and Sorger, B. (under review). Frequency-selective attention in auditory scenes recruits frequency representations throughout human superior temporal cortex.

Sengupta, S., Kemper, V.G., Roebroeck, A., Poser, B.A., Zimmermann, J., De Martino, F., Goebel, R., and Adriany, G. (in preparation). A 4 channel phased array Tx and 16 channel receive coil for high resolution visual cortical imaging at $7 \mathrm{~T}$.

Kemper, V.G., De Martino, F., Emmerling, T., Yacoub, E., and Goebel, R. (in preparation). 9.4 $\mathrm{T}$ anatomical and functional investigation of human primary and secondary visual cortex.

Emmerling, T.C., Valente, G., Zimmermann, J., Kemper, V.G., Frost, M., and Goebel, R. (in preparation). Overlapping feature representations in human visual cortex: Binocular disparity and axis-of-motion tuning mapped in human area MT using ultra-high field fMRI.

Moerel, M., De Martino, F., Kemper, V.G., Schmitter, S., Vu, A.T., Ugurbil, K., Formisano, E., and Yacoub, E. (in preparation) Sensitivity and specificity considerations for fMRI encoding, decoding, and mapping of voxel preferences at ultra-high field.

Reithler, J., Kashyap, S., Ivanov, D., Kemper, V.G., de Weerd, P., and Goebel, R. (in preparation) Linking hippocampal pattern separation to subsequent recognition memory performance: a $7 \mathrm{~T} \mathrm{fMRI} \mathrm{study.}$

\section{Conference Contributions}

Kemper, V.G., Oros-Peusquens, A-M., and Shah, N.J. (2011). Spatially resolved two-dimentional T1-T2 relaxometry in the human brain using inversion recovery spin-echo measurements and NNLS. 19th Annual Meeting of ISMRM, 2757. 
Poser, B.A., Kemper, V.G., Ivanov, D., Kannengiesser, S.A., and Barth, M. (2013). CAIPIRINHA-accelerated 3D EPI for high temporal and/or spatial resolution EPI acquisitions. Annual Meeting of ESMRMB, 287.

Riecke, L., Peters, J.C., Valente, G., Kemper, V.G., Formisano, E., and Sorger, B. Attention on tonotopy: Task- and stimulus-driven audiofrequency representations in human supratemporal cortex.

Plantinga, B., Roebroeck, A., Bastiani, M., Kemper, V.G., Kuijf, M., Uludağ, K., ter Haar Romeny, B., and Temel, Y. (2014). Ultra-high resolution ex vivo connectivity and parcellation of the human subthalamic nucleus; XXI Congress of the European Society of Stereotactic and Functional Neurosurgery.

Goebel, R., Vu, A.T., Kemper, V.G., Castelo-Branco, M., Ugurbil, K., de Martino, F., and Yacoub, E. (2014). Revealing columnar-level neural correlates of perceptual switches in area hMT using fMRI at 7 Tesla. Annual meeting of the society for neuroscience.

Moerel, M., De Martino, F., Vu, A.T., Kemper, V.G., Ugurbil, K., Formisano E., and Yacoub, E. (2014) Laminar and columnar features of cortical natural sound processing in humans. 22nd Annual Meeting of ISMRM.

Kemper, V.G., De Martino, F., Vu, A.T., Poser, B.A., Feinberg, D.A., Yacoub, E., and Goebel, R. (2014). Evaluation of Point Spread Function and Functional Sensitivity of 3D-GRASE and 2D Spin-Echo EPI for SubMillimeter-Resolution fMRI at 7 T. 22nd Annual Meeting of ISMRM, 7493.

Kemper, V.G., De Martino, F., Vu, A.T., Feinberg, D.A., Yacoub, E., and Goebel, R. (2014). Variable Flip Angle 3D-GRASE for Increased Spatial Coverage and Improved Point Spread Function in High Resolution fMRI at 7T. 22nd Annual Meeting of ISMRM, 7676.

Tse, D.H.Y., Boer, V.O., Kemper, V.G., Klomp, D.W.J., and Jansen, J.F.A. (2015). High resolution MR Spectroscopic imaging of the visual cortex at 9.4T with minimal chemical shift displacement artefact, 23nd Annual Meeting of ISMRM, 3204.

Kemper, V.G., De Martino, F., Tse, D.H., Poser, B.A., Yacoub, E., and Goebel, R. (2015). T2 weighted high-resolution fMRI in human visual cortex at $9.4 \mathrm{~T}$ using 3D-GRASE. 23rd Annual Meeting of ISMRM, 1944.

Plantinga, B., Roebroeck, A., Bastiani, M., Kemper, V.G., Kuijf, M., Uludağ, K., ter Haar Romeny, B., and Temel, Y. (2015). Structural Connectivity Mapping and Parcellation of the Human Subthalamic Nucleus Using Ultra-High Field Diffusion MRI. 23rd Annual Meeting of ISMRM, 3514. 
Sengupta, S., Adriany, G., Kemper, V.G., Zimmermann, J., Goebel, R., and Roebroeck, A. (2015). An Open 4ch. Transmit / 16 Ch. Receive Coil for High Resolution Occipital and Temporal Visual Cortex Imaging at 7T. 23rd Annual Meeting of ISMRM, 1777.

Schneider, M., Goebel, R., Vu, A.T., Kemper, V.G., Castelo-Branco, M., Ugurbil, K., De Martino, F., and Yacoub, E. (2015). Revealing columnar-level neural correlates of perceptual switches in area hMT using fMRI at 7 Tesla. Annual Meeting of the ASSC, 7.

Plantinga, B., Roebroeck, A., Bastiani, M., Kemper, V.G., Kuijf, M., Uludağ, K., Temel, Y., and ter Haar Romeny, B. (2015). High Resolution Imaging of the Human Subthalamic Nucleus Network. 2nd Belgian Neuroinformatics congress.

Schneider, M.*, Goebel, R.*, Kemper, V.G., Vu, A.T., Castelo Branco, M., Ugurbil, K., Yacoub, E., and De Martino, F. (2016). Responses in Mesoscopic Clusters of Human MT+ Reflect the Perceived Direction of Ambiguous Motion. Annual meeting of the organization for human brain mapping (* authors contributed equally).

Sousa, T., Kemper, V.G., Costa G., Duarte, J.V., Martins R., Goebel R., and Castelo-Branco, M. (2016). Long-range perceptual integration of visual motion revealed at high resolution $7 \mathrm{~T}$ fMRI. Annual meeting of the organization of human brain mapping.

Sengupta, S., Adriany, G., Kemper, V.G., Goebel, R., and Roebroeck, A. (2016). A larger subject Field-of-View and denser coil arrays at UHF: What do we gain?. 24th Annual Meeting of ISMRM, 3520. 


\section{References}

Abdollahi, R.O., Kolster, H., Glasser, M.F., Robinson, E.C., Coalson, T.S., Dierker, D., Jenkinson, M., Van Essen, D.C., and Orban, G.A. (2014). Correspondences between retinotopic areas and myelin maps in human visual cortex. Neuroimage 99, 509-524. doi: 10.1016/j.neuroimage.2014.06.042.

Adams, D.L., and Horton, J.C. (2009). Ocular dominance columns: enigmas and challenges. Neuroscientist 15, 62-77. doi: $10.1177 / 1073858408327806$.

Adams, D.L., Sincich, L.C., and Horton, J.C. (2007). Complete pattern of ocular dominance columns in human primary visual cortex. Journal of Neuroscience 27, 10391-10403. doi: 10.1523/jneurosci.292307.2007.

Andersson, J.L., Skare, S., and Ashburner, J. (2003). How to correct susceptibility distortions in spin-echo echo-planar images: application to diffusion tensor imaging. Neuroimage 20, 870-888. doi: 10.1016/s1053-8119(03)00336-7.

Annese, J., Pitiot, A., Dinov, I., and Toga, A. (2004). A myelo-architectonic method for the structural classification of cortical areas. Neuroimage 21, 15-26.

Attwell, D., Buchan, A.M., Charpak, S., Lauritzen, M., Macvicar, B.A., and Newman, E.A. (2010). Glial and neuronal control of brain blood flow. Nature 468, 232-243.

Auerbach, E.J., Heberlein, K., and Hu, X. (2002). High-resolution T2 fMRI at high magnetic fields using PSIF. Proceedings of the 10th Annual Meeting of ISMRM, Honolulu, Hawaii, 2345.

Bandettini, P.A., Wong, E.C., Hinks, R.S., Tikofsky, R.S., and Hyde, J.S. (1992). Time course EPI of human brain function during task activation. Magn Reson Med 25, 390-397. doi: 10.1002/mrm.1910250220.

Bandettini, P.A., Wong, E.C., Jesmanowicz, A., Hinks, R.S., and Hyde, J.S. (1994). Spin-Echo and Gradient-Echo Epi of Human Brain Activation Using Bold Contrast - a Comparative-Study at $1.5 \mathrm{~T}$. Nmr in Biomedicine 7, 12-20. doi: 10.1002/nbm.1940070104.

Barth, M., Meyer, H., Kannengiesser, S.A., Polimeni, J.R., Wald, L.L., and Norris, D.G. (2010). T2-weighted 3D fMRI using S2-SSFP at 7 tesla. Magn Reson Med 63, 1015-1020. doi: 10.1002/mrm.22283. 
Barth, M., and Norris, D.G. (2007). Very high-resolution three-dimensional functional MRI of the human visual cortex with elimination of large venous vessels. NMR Biomed 20, 477-484. doi: 10.1002/nbm.1158.

Bastiani, M., Oros-Peusquens, A.M., Seehaus, A., Brenner, D., Möllenhoff, K., Celik, A., Felder, J., Bratzke, H., Shah, N.J., Galuske, R., Goebel, R., and Roebroeck, A. (2015). "Automatic classification of human cortical layers and areas using high resolution diffusion MRI," in Multi-scale brain connectivity with diffusion MRI (phD thesis).).

Bause, J., Ehses, P., Mirkes, C., Shajan, G., Scheffler, K., and Pohmann, R. (2015). Quantitative and functional pulsed arterial spin labeling in the human brain at $9.4 \mathrm{t}$. Magnetic Resonance in Medicine.

Belliveau, J.W., Kennedy, D.N., Jr., Mckinstry, R.C., Buchbinder, B.R., Weisskoff, R.M., Cohen, M.S., Vevea, J.M., Brady, T.J., and Rosen, B.R. (1991). Functional mapping of the human visual cortex by magnetic resonance imaging. Science 254, 716-719.

Bernstein, M.A., King, K.F., and Zhou, X.J. (2004). Handbook of MRI pulse sequences. Elsevier.

Besle, J., Sanchez-Panchuelo, R.M., Bowtell, R., Francis, S., and Schluppeck, D. (2014). Event-related fMRI at 7T reveals overlapping cortical representations for adjacent fingertips in $\mathrm{S} 1$ of individual subjects. Hum Brain Mapp 35, 2027-2043. doi: 10.1002/hbm.22310.

Bianciardi, M., Fukunaga, M., Van Gelderen, P., De Zwart, J.A., and Duyn, J.H. (2011). Negative BOLD-fMRI signals in large cerebral veins. J Cereb Blood Flow Metab 31, 401-412. doi: 10.1038/jcbfm.2010.164.

Bieri, O., and Scheffler, K. (2007). Effect of diffusion in inhomogeneous magnetic fields on balanced steady-state free precession. NMR in Biomedicine 20, 1-10. doi: 10.1002/nbm.1079.

Bilgic, B., Gagoski, B.A., Cauley, S.F., Fan, A.P., Polimeni, J.R., Grant, P.E., Wald, L.L., and Setsompop, K. (2015). Wave-CAIPI for highly accelerated 3D imaging. Magnetic Resonance in Medicine 73, 21522162.

Birn, R.M., Smith, M.A., Jones, T.B., and Bandettini, P.A. (2008). The respiration response function: the temporal dynamics of $\mathrm{fMRI}$ signal fluctuations related to changes in respiration. Neuroimage 40, 644-654. doi: 10.1016/j.neuroimage.2007.11.059.

Blake, R., O'shea, R.P., and Mueller, T. (1992). Spatial zones of binocular rivalry in central and peripheral vision. Visual neuroscience 8,469 478.

Blamire, A.M., Ogawa, S., Ugurbil, K., Rothman, D., Mccarthy, G., Ellermann, J.M., Hyder, F., Rattner, Z., and Shulman, R.G. (1992). Dynamic mapping of the human visual cortex by high-speed 
magnetic resonance imaging. Proceedings of the National Academy of Sciences 89, 11069-11073.

Blasdel, G.G. (1992). Differential imaging of ocular dominance and orientation selectivity in monkey striate cortex. J Neurosci 12, 3115-3138.

Blasdel, G.G., and Salama, G. (1986). Voltage-sensitive dyes reveal a modular organization in monkey striate cortex. Nature 321, 579585.

Bonhoeffer, T., and Grinvald, A. (1991). Iso-orientation domains in cat visual cortex are arranged in pinwheel-like patterns. Nature 353, 429-431.

Boxerman, J.L., Bandettini, P.A., Kwong, K.K., Baker, J.R., Davis, T.L., Rosen, B.R., and Weisskoff, R.M. (1995a). The Intravascular Contribution to Fmri Signal Change - Monte-Carlo Modeling and DiffusionWeighted Studies in-Vivo. Magnetic Resonance in Medicine 34, 410. doi: DOI 10.1002/mrm.1910340103.

Boxerman, J.L., Hamberg, L.M., Rosen, B.R., and Weisskoff, R.M. (1995b). $\mathrm{Mr}$ Contrast Due to Intravascular Magnetic-Susceptibility Perturbations. Magnetic Resonance in Medicine 34, 555-566. doi: 10.1002/mrm.1910340412.

Boyacioglu, R., Schulz, J., Muller, N.C., Koopmans, P.J., Barth, M., and Norris, D.G. (2014). Whole brain, high resolution multiband spinecho EPI fMRI at 7T: A comparison with gradient-echo EPI using a color-word Stroop task. Neuroimage 97, 142-150. doi: 10.1016/j.neuroimage.2014.04.011.

Boynton, G.M., Engel, S.A., Glover, G.H., and Heeger, D.J. (1996). Linear systems analysis of functional magnetic resonance imaging in human V1. The journal of neuroscience 16, 4207-4221.

Breuer, F.A., Blaimer, M., Mueller, M.F., Seiberlich, N., Heidemann, R.M., Griswold, M.A., and Jakob, P.M. (2006). Controlled aliasing in volumetric parallel imaging (2D CAIPIRINHA). Magn Reson Med 55, 549-556. doi: 10.1002/mrm.20787.

Bridge, H., Clare, S., Jenkinson, M., Jezzard, P., Parker, A.J., and Matthews, P.M. (2005). Independent anatomical and functional measures of the V1/V2 boundary in human visual cortex. Journal of Vision 5, 1.

Broca, P. (1865). Sur le siège de la faculté du langage articulé. Bulletins de la Société d'Anthropologie de Paris 6, 377-393.

Brodmann, K. (1909). Vergleichende Lokalisationslehre der Groshirnrinde. Leipzig: Barth.

Buccino, G., Binkofski, F., Fink, G.R., Fadiga, L., Fogassi, L., Gallese, V., Seitz, R.J., Zilles, K., Rizzolatti, G., and Freund, H.J. (2001). Action observation activates premotor and parietal areas in a somatotopic 
manner: an fMRI study. European journal of neuroscience 13, 400404.

Budde, J., Shajan, G., Hoffmann, J., Ugurbil, K., and Pohmann, R. (2011). Human imaging at 9.4 $\mathrm{T}$ using $\mathrm{T}(2){ }^{*}$-, phase-, and susceptibilityweighted contrast. Magn Reson Med 65, 544-550. doi: 10.1002/mrm.22632.

Budde, J., Shajan, G., Scheffler, K., and Pohmann, R. (2014a). Ultra-high resolution imaging of the human brain using acquisition-weighted imaging at 9.4T. Neuroimage 86, 592-598. doi: 10.1016/j.neuroimage.2013.08.013.

Budde, J., Shajan, G., Zaitsev, M., Scheffler, K., and Pohmann, R. (2014b). Functional MRI in human subjects with gradient-echo and spinecho EPI at 9.4 T. Magnetic Resonance in Medicine 71, 209-218.

Busse, R.F., Brau, A.C., Vu, A., Michelich, C.R., Bayram, E., Kijowski, R., Reeder, S.B., and Rowley, H.A. (2008). Effects of refocusing flip angle modulation and view ordering in 3D fast spin echo. Magn Reson Med 60, 640-649. doi: 10.1002/mrm.21680.

Busse, R.F., Hariharan, H., Vu, A., and Brittain, J.H. (2006). Fast spin echo sequences with very long echo trains: design of variable refocusing flip angle schedules and generation of clinical T2 contrast. Magn Reson Med 55, 1030-1037. doi: 10.1002/mrm.20863.

Buxton, R.B. (2001). The elusive initial dip. Neuroimage 13, 953-958.

Buxton, R.B. (2012). Dynamic models of BOLD contrast. Neurolmage 62, 953-961. doi: 10.1016/j.neuroimage.2012.01.012.

Buxton, R.B., Wong, E.C., and Frank, L.R. (1998). Dynamics of blood flow and oxygenation changes during brain activation: the balloon model. Magnetic resonance in medicine 39, 855-864.

Callaghan, M.F., Josephs, O., Herbst, M., Zaitsev, M., Todd, N., and Weiskopf, N. (2015). An evaluation of prospective motion correction (PMC) for high resolution quantitative MRI. Frontiers in Neuroscience 9, 97. doi: 10.3389/fnins.2015.00097.

Chaimow, D., Yacoub, E., Ugurbil, K., and Shmuel, A. (2011). Modeling and analysis of mechanisms underlying fMRI-based decoding of information conveyed in cortical columns. Neuroimage 56, 627642. doi: 10.1016/j.neuroimage.2010.09.037.

Chang, C., and Glover, G.H. (2009). Relationship between respiration, endtidal CO2, and BOLD signals in resting-state fMRI. Neuroimage 47, 1381-1393. doi: 10.1016/j.neuroimage.2009.04.048.

Chen, L., and Feinberg, D. (Year). "Simultaneous Multi-Volume GRASE Imaging", in: Proceedings of the 21st Annual Meeting of ISMRM), 2365. 
Cheng, K., Waggoner, R.A., and Tanaka, K. (2001). Human ocular dominance columns as revealed by high-field functional magnetic resonance imaging. Neuron 32, 359-374. doi: 10.1016/s08966273(01)00477-9.

Cho, Z., and Ro, Y. (1992). Reduction of susceptibility artifact in gradientecho imaging. Magnetic resonance in medicine 23, 193-200.

Chung, J.Y., In, M.H., Oh, S.H., Zaitsev, M., Speck, O., and Cho, Z.H. (2011). An improved PSF mapping method for EPI distortion correction in human brain at ultra high field (7T). MAGMA 24, 179-190. doi: 10.1007/s10334-011-0251-1.

Clare, S., and Jezzard, P. (2001). Rapid T(1) mapping using multislice echo planar imaging. Magn Reson Med 45, 630-634.

Constable, R.T., and Gore, J.C. (1992). The loss of small objects in variable TE imaging: implications for FSE, RARE, and EPI. Magn Reson Med 28, 9-24. doi: 10.1002/mrm.1910280103.

Constable, R.T., Kennan, R.P., Puce, A., Mccarthy, G., and Gore, J.C. (1994). Functional NMR imaging using fast spin echo at $1.5 \mathrm{~T}$. Magn Reson Med 31, 686-690. doi: 10.1002/mrm.1910310617.

Cox, E.F., and Gowland, P.A. (2010). Simultaneous quantification of T2 and T'2 using a combined gradient echo-spin echo sequence at ultrahigh field. Magn Reson Med 64, 1440-1445. doi: 10.1002/mrm.22522.

Cusack, R., Brett, M., and Osswald, K. (2003). An evaluation of the use of magnetic field maps to undistort echo-planar images. Neuroimage $18,127-142$.

Da Costa, N.M., and Martin, K.A. (2010). Whose Cortical Column Would that Be? Front Neuroanat 4, 16. doi: 10.3389/fnana.2010.00016.

Damadian, R. (1971). Tumor detection by nuclear magnetic resonance. Science 171, 1151-1153.

Damadian, R., Minkoff, L., and Goldsmith, M. (1977). NMR in cancer: XXI. FONAR scan of the live human abdomen. Physiological chemistry and physics 10, 561-563.

Damasio, H., Grabowski, T., Frank, R., Galaburda, A.M., and Damasio, A.R. (1994). The return of Phineas Gage: clues about the brain from the skull of a famous patient. Science 264, 1102-1105.

De Martino, F., Esposito, F., Van De Moortele, P.F., Harel, N., Formisano, E., Goebel, R., Ugurbil, K., and Yacoub, E. (2011). Whole brain highresolution functional imaging at ultra high magnetic fields: an application to the analysis of resting state networks. Neuroimage 57, 1031-1044. doi: 10.1016/j.neuroimage.2011.05.008.

De Martino, F., Moerel, M., Xu, J., Van De Moortele, P.F., Ugurbil, K., Goebel, R., Yacoub, E., and Formisano, E. (2014). High-Resolution 
Mapping of Myeloarchitecture In Vivo: Localization of Auditory Areas in the Human Brain. Cereb Cortex. doi: 10.1093/cercor/bhu150.

De Martino, F., Schmitter, S., Moerel, M., Tian, J., Ugurbil, K., Formisano, E., Yacoub, E., and Van De Moortele, P.-F. (2012). Spin echo functional MRI in bilateral auditory cortices at $7 \mathrm{~T}$ : An application of B-1 shimming. Neuroimage 63, 1313-1320. doi: 10.1016/j.neuroimage.2012.08.029.

De Martino, F., Zimmermann, J., Muckli, L., Ugurbil, K., Yacoub, E., and Goebel, R. (2013). Cortical depth dependent functional responses in humans at 7T: improved specificity with 3D GRASE. PLoS One 8, e60514. doi: 10.1371/journal.pone.0060514.

Dechent, P., and Frahm, J. (2000). Direct mapping of ocular dominance columns in human primary visual cortex. Neuroreport 11, 32473249.

Deichmann, R., Good, C.D., Josephs, O., Ashburner, J., and Turner, R. (2000). Optimization of 3-D MP-RAGE sequences for structural brain imaging. Neuroimage 12, 112-127. doi: 10.1006/nimg.2000.0601.

Deistung, A., Rauscher, A., Sedlacik, J., Stadler, J., Witoszynskyj, S., and Reichenbach, J.R. (2008). Susceptibility weighted imaging at ultra high magnetic field strengths: theoretical considerations and experimental results. Magnetic Resonance in Medicine 60, 11551168. doi: 10.1002/mrm.21754.

Deyoe, E.A., Carman, G.J., Bandettini, P., Glickman, S., Wieser, J., Cox, R., Miller, D., and Neitz, J. (1996). Mapping striate and extrastriate visual areas in human cerebral cortex. Proceedings of the National Academy of Sciences 93, 2382-2386.

Donahue, M.J., Stevens, R.D., De Boorder, M., Pekar, J.J., Hendrikse, J., and Van Zijl, P.C. (2009). Hemodynamic changes after visual stimulation and breath holding provide evidence for an uncoupling of cerebral blood flow and volume from oxygen metabolism. Journal of Cerebral Blood Flow \& Metabolism 29, 176-185.

Douglas, R.J., and Martin, K. (1991). A functional microcircuit for cat visual cortex. The Journal of Physiology 440, 735-769.

Douglas, R.J., and Martin, K.A. (2004). Neuronal circuits of the neocortex. Annu. Rev. Neurosci. 27, 419-451.

Downing, P.E., Jiang, Y., Shuman, M., and Kanwisher, N. (2001). A cortical area selective for visual processing of the human body. Science 293, 2470-2473. doi: 10.1126/science.1063414.

Dragonu, I., Lange, T., Baxan, N., Snyder, J., Hennig, J., and Zaitsev, M. (2013). Accelerated point spread function mapping using signal 
modeling for accurate echo-planar imaging geometric distortion correction. Magn Reson Med 69, 1650-1656. doi: 10.1002/mrm.24396.

Dumoulin, S.O., and Wandell, B.A. (2008). Population receptive field estimates in human visual cortex. Neuroimage 39, 647-660. doi: 10.1016/j.neuroimage.2007.09.034.

Duong, T.Q., Kim, D.S., Ugurbil, K., and Kim, S.G. (2001). Localized cerebral blood flow response at submillimeter columnar resolution. Proc Natl Acad Sci U S A 98, 10904-10909. doi: 10.1073/pnas.191101098.

Duong, T.Q., Kim, D.S., Uğurbil, K., and Kim, S.G. (2000). Spatiotemporal dynamics of the BOLD fMRI signals: toward mapping submillimeter cortical columns using the early negative response. Magnetic resonance in medicine 44, 231-242.

Duong, T.Q., Yacoub, E., Adriany, G., Hu, X., Ugurbil, K., and Kim, S.G. (2003). Microvascular BOLD contribution at 4 and $7 \mathrm{~T}$ in the human brain: gradient-echo and spin-echo fMRI with suppression of blood effects. Magn Reson Med 49, 1019-1027. doi: 10.1002/mrm.10472.

Duong, T.Q., Yacoub, E., Adriany, G., Hu, X., Ugurbil, K., Vaughan, J.T., Merkle, H., and Kim, S.G. (2002). High-resolution, spin-echo BOLD, and CBF FMRI at 4 and 7 T. Magn Reson Med 48, 589-593.

Duvernoy, H.M., Delon, S., and Vannson, J. (1981). Cortical blood vessels of the human brain. Brain research bulletin 7, 519-579.

Duyn, J.H., Van Gelderen, P., Li, T.-Q., De Zwart, J.A., Koretsky, A.P., and Fukunaga, M. (2007). High-field MRI of brain cortical substructure based on signal phase. Proceedings of the National Academy of Sciences of the United States of America 104, 11796-11801. doi: 10.1073/pnas.0610821104.

Edelstein, W.A., Glover, G.H., Hardy, C.J., and Redington, R.W. (1986). The intrinsic signal-to-noise ratio in NMR imaging. Magn Reson Med 3, 604-618. doi: 10.1002/mrm.1910030413.

Ehses, P., Bause, J., Shajan, G., and Scheffler, K. (2014). Efficient generation of $\mathrm{T}^{*}$-weighted contrast by interslice echo-shifting for human functional and anatomical imaging at 9.4 Tesla. Magnetic Resonance in Medicine.

Ehses, P., Budde, J., Shajan, G., and Scheffler, K. (2013). T2-weighted BOLD fMRI at 9.4 T using a S2-SSFP-EPI sequence. 21st Annual Meeting of ISMRM 21, 0414.

Eichfelder, G., and Gebhardt, M. (2011). Local specific absorption rate control for parallel transmission by virtual observation points. Magnetic Resonance in Medicine 66, 1468-1476. 
Engel, S.A., Glover, G.H., and Wandell, B.A. (1997). Retinotopic organization in human visual cortex and the spatial precision of functional MRI. Cerebral cortex 7, 181-192.

Engel, S.A., Rumelhart, D.E., Wandell, B.A., Lee, A.T., Glover, G.H., Chichilnisky, E.J., and Shadlen, M.N. (1994). fMRI of human visual cortex. Nature 369, 525. doi: 10.1038/369525a0.

Epstein, R., and Kanwisher, N. (1998). A cortical representation of the local visual environment. Nature 392, 598-601.

Ernst, T., and Hennig, J. (1994). Observation of a fast response in functional MR. Magnetic resonance in medicine 32, 146-149.

Feinberg, D.A., Hale, J.D., Watts, J.C., Kaufman, L., and Mark, A. (1986). Halving MR imaging time by conjugation: demonstration at $3.5 \mathrm{kG}$. Radiology 161, 527-531. doi: 10.1148/radiology.161.2.3763926.

Feinberg, D.A., Harel, N., Ramanna, S., Ugurbil, K., and Yacoub, E. (2008). Sub-millimeter Single-shot 3D GRASE with Inner Volume Selection for T2 weighted fMRI applications at 7 Tesla. 16th Annual Meeting International Society for Magnetic Resonance in Medicine, 2373.

Feinberg, D.A., Hoenninger, J.C., Crooks, L.E., Kaufman, L., Watts, J.C., and Arakawa, M. (1985). Inner volume MR imaging: technical concepts and their application. Radiology 156, 743-747. doi: 10.1148/radiology.156.3.4023236.

Feinberg, D.A., Moeller, S., Smith, S.M., Auerbach, E., Ramanna, S., Gunther, M., Glasser, M.F., Miller, K.L., Ugurbil, K., and Yacoub, E. (2010). Multiplexed echo planar imaging for sub-second whole brain FMRI and fast diffusion imaging. PLoS One 5, e15710. doi: 10.1371/journal.pone.0015710.

Feinberg, D.A., and Oshio, K. (1991). GRASE (gradient- and spin-echo) MR imaging: a new fast clinical imaging technique. Radiology 181, 597602. doi: 10.1148/radiology.181.2.1924811.

Felleman, D.J., and Van Essen, D.C. (1991). Distributed hierarchical processing in the primate cerebral cortex. Cereb Cortex 1, 1-47.

Fernandez-Seara, M.A., Wang, Z., Wang, J., Rao, H.Y., Guenther, M., Feinberg, D.A., and Detre, J.A. (2005). Continuous arterial spin labeling perfusion measurements using single shot 3D GRASE at 3 T. Magn Reson Med 54, 1241-1247. doi: 10.1002/mrm.20674.

Finsterbusch, J. (2010). Fast-spin-echo imaging of inner fields-of-view with 2D-selective RF excitations. J Magn Reson Imaging 31, 1530-1537. doi: 10.1002/jmri.22196.

Finsterbusch, J. (2013). Functional neuroimaging of inner fields-of-view with 2D-selective RF excitations. Magnetic Resonance Imaging 31, 1228-1235. doi: 10.1016/j.mri.2013.03.005. 
Formisano, E., Kim, D.S., Di Salle, F., Van De Moortele, P.F., Ugurbil, K., and Goebel, R. (2003). Mirror-symmetric tonotopic maps in human primary auditory cortex. Neuron 40, 859-869.

Forstmann, B.U., Keuken, M.C., Schafer, A., Bazin, P.-L., Alkemade, A., and Turner, R. (2014). Multi-modal ultra-high resolution structural 7Tesla MRI data repository. Scientific data 1.

Fox, P.T., and Raichle, M.E. (1986). Focal physiological uncoupling of cerebral blood flow and oxidative metabolism during somatosensory stimulation in human subjects. Proceedings of the National Academy of Sciences 83, 1140-1144.

Frahm, J., Bruhn, H., Merboldt, K.D., and Hänicke, W. (1992). Dynamic MR imaging of human brain oxygenation during rest and photic stimulation. Journal of Magnetic Resonance Imaging 2, 501-505.

Frahm, J., Haase, A., and Matthaei, D. (1986). Rapid three-dimensional MR imaging using the FLASH technique. Journal of computer assisted tomography 10, 363-368.

Freeman, J., Brouwer, G.J., Heeger, D.J., and Merriam, E.P. (2011). Orientation decoding depends on maps, not columns. J Neurosci 31, 4792-4804. doi: 10.1523/JNEUROSCI.5160-10.2011.

Friston, K.J., Fletcher, P., Josephs, O., Holmes, A., Rugg, M.D., and Turner, R. (1998). Event-related fMRI: characterizing differential responses. Neuroimage 7, 30-40. doi: 10.1006/nimg.1997.0306.

Fritz, L., Mulders, J., Bremann, H., Peters, J., Bastiani, M., Roebroeck, A., Andersson, J.L., Ashburner, J., Weiskopf, N., and Goebel, R. (Year). "Comparison of EPI distortion correction methods at 3T and 7T", in: OHBM Annual Meeting).

Frost, M.A., and Goebel, R. (2012). Measuring structural-functional correspondence: spatial variability of specialised brain regions after macro-anatomical alignment. Neuroimage 59, 1369-1381. doi: 10.1016/j.neuroimage.2011.08.035.

Fukunaga, M., Li, T.-Q., Van Gelderen, P., De Zwart, J.A., Shmueli, K., Yao, B., Lee, J., Maric, D., Aronova, M.A., Zhang, G., Leapman, R.D., Schenck, J.F., Merkle, H., and Duyn, J.H. (2010). Layer-specific variation of iron content in cerebral cortex as a source of MRI contrast. Proceedings of the National Academy of Sciences of the United States of America 107, 3834-3839. doi: 10.1073/pnas.0911177107.

Garcia, D. (2010). Robust smoothing of gridded data in one and higher dimensions with missing values. Computational statistics \& data analysis 54, 1167-1178. 
Gardner, J.L. (2010). Is cortical vasculature functionally organized? Neuroimage 49, 1953-1956. doi: 10.1016/j.neuroimage.2009.07.004.

Gati, J.S., Menon, R.S., Uğurbil, K., and Rutt, B.K. (1997). Experimental determination of the BOLD field strength dependence in vessels and tissue. Magnetic resonance in medicine 38, 296-302.

Geißler, A., Fischmeister, F.P.S., Grabner, G., Wurnig, M., Rath, J., Foki, T., Matt, E., Trattnig, S., Beisteiner, R., and Robinson, S.D. (2013). Comparing the microvascular specificity of the 3-and 7-T BOLD response using ICA and susceptibility-weighted imaging. Frontiers in human neuroscience 7.

Gilbert, C.D. (1983). Microcircuitry of the visual cortex. Annual review of neuroscience 6, 217-247.

Glasser, M.F., and Van Essen, D.C. (2011). Mapping human cortical areas in vivo based on myelin content as revealed by T1-and T2-weighted MRI. The Journal of Neuroscience 31, 11597-11616.

Glover, G.H., Li, T.Q., and Ress, D. (2000). Image-based method for retrospective correction of physiological motion effects in $\mathrm{fMRI}$ : RETROICOR. Magnetic Resonance in Medicine 44, 162-167.

Goa, P.E., Koopmans, P.J., Poser, B.A., Barth, M., and Norris, D.G. (2014). BOLD fMRI signal characteristics of S1- and S2-SSFP at 7 Tesla. Frontiers in Neuroscience 8. doi: 10.3389/fnins.2014.00049.

Goa, P.E., Poser, B.A., and Barth, M. (2013). Modeling and suppression of respiration induced $\mathrm{B} 0$-fluctuations in non-balanced steady-state free precession sequences at 7 Tesla. Magnetic Resonance Materials in Physics Biology and Medicine 26, 377-387. doi: 10.1007/s10334-012-0343-6.

Goebel, R. (2015). "Functional Organization of the Primary Visual Cortex," in Brain Mapping: An Encyclopedic Reference, ed. A.W. Toga. Academic Press: Elsevier), 287-291.

Goebel, R., Khorram-Sefat, D., Muckli, L., Hacker, H., and Singer, W. (1998). The constructive nature of vision: direct evidence from functional magnetic resonance imaging studies of apparent motion and motion imagery. Eur J Neurosci 10, 1563-1573.

Goense, J., Merkle, H., and Logothetis, N.K. (2012). High-resolution fMRI reveals laminar differences in neurovascular coupling between positive and negative BOLD responses. Neuron 76, 629-639. doi: 10.1016/j.neuron.2012.09.019.

Goense, J.B., and Logothetis, N.K. (2006a). Laminar specificity in monkey V1 using high-resolution SE-fMRI. Magn Reson Imaging 24, 381-392. doi: 10.1016/j.mri.2005.12.032. 
Goense, J.B.M., and Logothetis, N.K. (2006b). Laminar specificity in monkey V1 using high-resolution SE-fMRI. Magnetic Resonance Imaging 24, 381-392. doi: 10.1016/j.mri.2005.12.032.

Goerke, U., Garwood, M., and Ugurbil, K. (2011). Functional magnetic resonance imaging using RASER. Neuroimage 54, 350-360.

Goerke, U., Van De Moortele, P.F., and Ugurbil, K. (2007). Enhanced relative BOLD signal changes in $\mathrm{T}(2)$-weighted stimulated echoes. Magn Reson Med 58, 754-762. doi: 10.1002/mrm.21369.

Gomori, J., Holland, G., Grossman, R., Gefter, W., and Lenkinski, R. (1988). Fat suppression by section-select gradient reversal on spin-echo MR imaging. Work in progress. Radiology 168, 493-495. doi: 10.1148/radiology.168.2.3393670.

Goncalves, N.R., Ban, H., Sanchez-Panchuelo, R.M., Francis, S.T., Schluppeck, D., and Welchman, A.E. (2015). 7 tesla FMRI reveals systematic functional organization for binocular disparity in dorsal visual cortex. J Neurosci 35, 3056-3072. doi: 10.1523/jneurosci.3047-14.2015.

Gonzalez-Castillo, J., Roopchansingh, V., Bandettini, P.A., and Bodurka, J. (2011). Physiological noise effects on the flip angle selection in BOLD fMRI. Neuroimage 54, 2764-2778. doi: 10.1016/j.neuroimage.2010.11.020.

Goodyear, B.G., and Menon, R.S. (2001). Brief visual stimulation allows mapping of ocular dominance in visual cortex using fMRI. Human Brain Mapping 14, 210-217. doi: 10.1002/hbm.1053.

Grinvald, A., Frostig, R.D., Siegel, R.M., and Bartfeld, E. (1991). Highresolution optical imaging of functional brain architecture in the awake monkey. Proceedings of the National Academy of Sciences 88, 11559-11563.

Grinvald, A., Lieke, E., Frostig, R.D., Gilbert, C.D., and Wiesel, T.N. (1986). Functional architecture of cortex revealed by optical imaging of intrinsic signals.

Griswold, M.A., Jakob, P.M., Heidemann, R.M., Nittka, M., Jellus, V., Wang, J., Kiefer, B., and Haase, A. (2002). Generalized autocalibrating partially parallel acquisitions (GRAPPA). Magn Reson Med 47, 1202-1210. doi: $10.1002 / \mathrm{mrm} .10171$.

Gu, H., Feng, H., Zhan, W., Xu, S., Silbersweig, D.A., Stern, E., and Yang, Y. (2002). Single-shot interleaved z-shim EPI with optimized compensation for signal losses due to susceptibility-induced field inhomogeneity at 3 T. Neuroimage 17, 1358-1364.

Haacke, E., Lindskogj, E., and Lin, W. (1991). A fast, iterative, partial-Fourier technique capable of local phase recovery. Journal of Magnetic Resonance (1969) 92, 126-145. 
Haacke, E.M., Brown, R., Thompson, M., and Venkatesan, R. (1999). Magnetic resonance imaging: physical principles and sequence design. 1999. New York: A John Wiley and Sons.

Hahn, E. (1950a). Nuclear induction due to free Larmor precession. Physical Review 77, 297.

Hahn, E.L. (1950b). Spin echoes. Physical review 80, 580.

Hall, C.N., Reynell, C., Gesslein, B., Hamilton, N.B., Mishra, A., Sutherland, B.A., O'farrell, F.M., Buchan, A.M., Lauritzen, M., and Attwell, D. (2014). Capillary pericytes regulate cerebral blood flow in health and disease. Nature 508, 55-60. doi: 10.1038/nature13165.

Harel, N., Lin, J., Moeller, S., Ugurbil, K., and Yacoub, E. (2006). Combined imaging-histological study of cortical laminar specificity of fMRI signals. Neuroimage 29, 879-887. doi: 10.1016/j.neuroimage.2005.08.016.

Harlow, J.M. (1868). Recovery from the passage of an iron bar through the head. Publications of the Massachusetts Medical Society 2, 327246.

Harmer, J., Sanchez-Panchuelo, R.M., Bowtell, R., and Francis, S.T. (2012). Spatial location and strength of BOLD activation in high-spatialresolution $\mathrm{fMRI}$ of the motor cortex: a comparison of spin echo and gradient echo fMRI at 7 T. NMR Biomed 25, 717-725. doi: 10.1002/nbm.1783.

Harrison, R.V., Harel, N., Panesar, J., and Mount, R.J. (2002). Blood capillary distribution correlates with hemodynamic-based functional imaging in cerebral cortex. Cerebral Cortex 12, 225-233.

Haxby, J.V., Gobbini, M.I., Furey, M.L., Ishai, A., Schouten, J.L., and Pietrini, P. (2001). Distributed and overlapping representations of faces and objects in ventral temporal cortex. Science 293, 2425-2430.

Heidemann, R.M., Ivanov, D., Trampel, R., Fasano, F., Meyer, H., Pfeuffer, J., and Turner, R. (2012). Isotropic submillimeter fMRI in the human brain at $7 \mathrm{~T}$ : combining reduced field-of-view imaging and partially parallel acquisitions. Magn Reson Med 68, 1506-1516. doi: $10.1002 / \mathrm{mrm} .24156$.

Hennig, J. (1988). Multiecho imaging sequences with low refocusing flip angles. Journal of Magnetic Resonance (1969) 78, 397-407.

Hennig, J., Nauerth, A., and Friedburg, H. (1986). RARE imaging: a fast imaging method for clinical MR. Magn Reson Med 3, 823-833. doi: 10.1002/mrm.1910030602.

Hennig, J., Weigel, M., and Scheffler, K. (2003). Multiecho sequences with variable refocusing flip angles: optimization of signal behavior using smooth transitions between pseudo steady states (TRAPS). Magn Reson Med 49, 527-535. doi: 10.1002/mrm.10391. 
Hinds, O., Polimeni, J.R., Rajendran, N., Balasubramanian, M., Amunts, K., Zilles, K., Schwartz, E.L., Fischl, B., and Triantafyllou, C. (2009). Locating the functional and anatomical boundaries of human primary visual cortex. Neuroimage 46, 915-922.

Hinds, O.P., Rajendran, N., Polimeni, J.R., Augustinack, J.C., Wiggins, G., Wald, L.L., Rosas, H.D., Potthast, A., Schwartz, E.L., and Fischl, B. (2008). Accurate prediction of V1 location from cortical folds in a surface coordinate system. Neuroimage 39, 1585-1599.

Horton, J., and Hedley-Whyte, E.T. (1984). Mapping of cytochrome oxidase patches and ocular dominance columns in human visual cortex. Philosophical Transactions of the Royal Society of London. Series B, Biological Sciences, 255-272.

Horton, J.C. (1984). Cytochrome oxidase patches: a new cytoarchitectonic feature of monkey visual cortex. Philosophical Transactions of the Royal Society of London. Series B, Biological Sciences, 199-253.

Horton, J.C., and Adams, D.L. (2005). The cortical column: a structure without a function. Philos Trans R Soc Lond B Biol Sci 360, 837-862. doi: 10.1098/rstb.2005.1623.

Horton, J.C., Dagi, L.R., Mccrane, E.P., and De Monasterio, F.M. (1990). Arrangement of ocular dominance columns in human visual cortex. Arch Ophthalmol 108, 1025-1031.

Hu, X., Le, T.H., Parrish, T., and Erhard, P. (1995). Retrospective estimation and correction of physiological fluctuation in functional MRI. Magnetic resonance in medicine 34, 201-212.

$\mathrm{Hu}, \mathrm{X}$. , and Yacoub, E. (2012). The story of the initial dip in fMRI. Neurolmage 62, 1103-1108. doi: 10.1016/j.neuroimage.2012.03.005.

Hua, J., Jones, C.K., Qin, Q., and Van Zijl, P.C. (2013a). Implementation of vascular-space-occupancy MRI at 7T. Magn Reson Med 69, 10031013. doi: $10.1002 / \mathrm{mrm} .24334$.

Hua, J., Qin, Q., Van Zijl, P.C., Pekar, J.J., and Jones, C.K. (2013b). Wholebrain three-dimensional T2-weighted BOLD functional magnetic resonance imaging at 7 Tesla. Magn Reson Med. doi: 10.1002/mrm.25055.

Hubel, D., and Wiesel, T. (1963). Shape and arrangement of columns in cat's striate cortex. The Journal of physiology 165, 559-568.

Hubel, D.H., and Wiesel, T.N. (1962). Receptive fields, binocular interaction and functional architecture in the cat's visual cortex. The Journal of physiology 160, 106.

Hubel, D.H., and Wiesel, T.N. (1965). Receptive fields and functional architecture in two nonstriate visual areas (18 and 19) of the cat. Journal of neurophysiology 28, 229-289. 
Hubel, D.H., and Wiesel, T.N. (1968). Receptive fields and functional architecture of monkey striate cortex. The Journal of physiology 195, 215-243.

Hubel, D.H., and Wiesel, T.N. (1974). Sequence regularity and geometry of orientation columns in the monkey striate cortex. Journal of Comparative Neurology 158, 267-293.

Hubel, D.H., and Wiesel, T.N. (1977). Ferrier lecture: Functional architecture of macaque monkey visual cortex. Proceedings of the Royal Society of London B: Biological Sciences 198, 1-59.

Huber, L., Goense, J., Kennerley, A.J., Trampel, R., Guidi, M., Reimer, E., Ivanov, D., Neef, N., Gauthier, C.J., Turner, R., and Moller, H.E. (2015a). Cortical lamina-dependent blood volume changes in human brain at 7 T. Neuroimage 107, 23-33. doi: 10.1016/j.neuroimage.2014.11.046.

Huber, L., Guidi, M., Goense, J., Mildner, T., Trampel, R., Schulz, J., Eichner, C., Turner, R., and Möller, H.E. (2015b). The magnitude point spread function is an inadequate measure of T2*-blurring in EPI. 23nd Annual Meeting of ISMRM 23, 2056.

Huber, L., Ivanov, D., Krieger, S.N., Streicher, M.N., Mildner, T., Poser, B.A., Moller, H.E., and Turner, R. (2013). Slab-selective, BOLD-corrected VASO at 7 tesla provides measures of cerebral blood volume reactivity with high signal-to-noise ratio. Magn Reson Med. doi: 10.1002/mrm.24916.

Hutton, C., Bork, A., Josephs, O., Deichmann, R., Ashburner, J., and Turner, R. (2002). Image distortion correction in fMRI: A quantitative evaluation. Neuroimage 16, 217-240. doi: 10.1006/nimg.2001.1054.

Ivanov, D., Schafer, A., Streicher, M.N., Heidemann, R.M., Trampel, R., and Turner, R. (2010). A simple low-SAR technique for chemical-shift selection with high-field spin-echo imaging. Magn Reson Med 64, 319-326. doi: 10.1002/mrm.22518.

Jin, T., and Kim, S.-G. (2008a). Cortical layer-dependent dynamic blood oxygenation, cerebral blood flow and cerebral blood volume responses during visual stimulation. Neuroimage 43, 1-9. doi: 10.1016/j.neuroimage.2008.06.029.

Jin, T., and Kim, S.G. (2008b). Improved cortical-layer specificity of vascular space occupancy fMRI with slab inversion relative to spin-echo BOLD at 9.4 T. Neuroimage 40, 59-67. doi: 10.1016/j.neuroimage.2007.11.045.

Jones, S.E., Buchbinder, B.R., and Aharon, I. (2000). Three-dimensional mapping of cortical thickness using Laplace's equation. Human 
Brain Mapping 11, 12-32. doi: 10.1002/10970193(200009)11:1<12::aid-hbm20>3.0.co;2-k.

Kamitani, Y., and Tong, F. (2005). Decoding the visual and subjective contents of the human brain. Nat Neurosci 8, 679-685. doi: $10.1038 / \mathrm{nn} 1444$.

Kanwisher, N., Mcdermott, J., and Chun, M.M. (1997). The fusiform face area: a module in human extrastriate cortex specialized for face perception. The Journal of Neuroscience 17, 4302-4311.

Katscher, U., Bornert, P., Leussler, C., and Van Den Brink, J.S. (2003). Transmit SENSE. Magn Reson Med 49, 144-150. doi: 10.1002/mrm.10353.

Katz, L.C., Gilbert, C.D., and Wiesel, T.N. (1989). Local circuits and ocular dominance columns in monkey striate cortex. The Journal of Neuroscience 9, 1389-1399.

Kemper, V.G., De Martino, F., Tse, D.H., Poser, B.A., Yacoub, E., and Goebel, R. (2015a). T2 weighted high-resolution $\mathrm{fMRI}$ in human visual cortex at 9.4 T using 3D-GRASE. 23nd Annual Meeting of ISMRM 23, 1944.

Kemper, V.G., De Martino, F., Vu, A.T., Poser, B.A., Feinberg, D.A., Goebel, R., and Yacoub, E. (2015b). Sub-Millimeter T2 Weighted fMRI at 7 $\mathrm{T}$ : Comparison of 3D-GRASE and 2D SE-EPI. Frontiers in Neuroscience 9. doi: 10.3389/fnins.2015.00163.

Kemper, V.G., De Martino, F., Yacoub, E., and Goebel, R. (2015c). Variable flip angle 3D-GRASE for high resolution $\mathrm{fMRI}$ at 7 tesla. Magn Reson Med. doi: 10.1002/mrm.25979.

Kim, H., Kim, D.H., and Park, J. (2015). Variable-flip-angle single-slab 3D GRASE imaging with phase-independent image reconstruction. Magn Reson Med 73, 1041-1052. doi: 10.1002/mrm.25223.

Koning, W., Bluemink, J.J., Langenhuizen, E.A., Raaijmakers, A.J., Andreychenko, A., Van Den Berg, C.A., Luijten, P.R., Zwanenburg, J.J., and Klomp, D.W. (2013). High-resolution MRI of the carotid arteries using a leaky waveguide transmitter and a high-density receive array at 7 T. Magn Reson Med 69, 1186-1193. doi: 10.1002/mrm.24345.

Koopmans, P.J., Barth, M., and Norris, D.G. (2010). Layer-Specific BOLD Activation in Human VI. Human Brain Mapping 31, 1297-1304. doi: 10.1002/hbm.20936.

Koopmans, P.J., Barth, M., Orzada, S., and Norris, D.G. (2011). Multi-echo $\mathrm{fMRI}$ of the cortical laminae in humans at $7 \mathrm{~T}$. Neuroimage 56, 1276-1285. doi: 10.1016/j.neuroimage.2011.02.042.

Kumar, A., Welti, D., and Ernst, R. (1975). Imaging of macroscopic objects by NMR Fourier zeugmatography. Naturwissenschaften 62, 34-34. 
Kundu, P., Inati, S.J., Evans, J.W., Luh, W.M., and Bandettini, P.A. (2012). Differentiating BOLD and non-BOLD signals in $\mathrm{FMRI}$ time series using multi-echo EPI. Neuroimage 60, 1759-1770. doi: 10.1016/j.neuroimage.2011.12.028.

Kwong, K.K., Belliveau, J.W., Chesler, D.A., Goldberg, I.E., Weisskoff, R.M., Poncelet, B.P., Kennedy, D.N., Hoppel, B.E., Cohen, M.S., Turner, R., and Et Al. (1992). Dynamic magnetic resonance imaging of human brain activity during primary sensory stimulation. Proc Natl Acad Sci U S A 89, 5675-5679. doi: 10.1073/pnas.89.12.5675.

Larkman, D.J., Hajnal, J.V., Herlihy, A.H., Coutts, G.A., Young, I.R., and Ehnholm, G. (2001). Use of multicoil arrays for separation of signal from multiple slices simultaneously excited. Journal of Magnetic Resonance Imaging 13, 313-317.

Lauterbur, P.C. (1973). Image formation by induced local interactions: examples employing nuclear magnetic resonance. Nature 242, 190 191.

Lauterbur, P.C. (1974). Magnetic resonance zeugmatography. Pure and Applied Chemistry 40, 149-157.

Lee, J., Hirano, Y., Fukunaga, M., Silva, A.C., and Duyn, J.H. (2010). On the contribution of deoxy-hemoglobin to MRI gray-white matter phase contrast at high field. Neuroimage 49, 193-198.

Lee, S.-P., Silva, A.C., Ugurbil, K., and Kim, S.-G. (1999). Diffusion-weighted spin-echo $\mathrm{fMRI}$ at $9.4 \mathrm{~T}$ : microvascular/tissue contribution to BOLD signal changes. Magnetic Resonance in Medicine 42, 919-928. doi: 10.1002/(SICI)1522-2594(199911)42:5<919::AID-MRM12>3.0.CO;28.

Levay, S., Connolly, M., Houde, J., and Van Essen, D. (1985). The complete pattern of ocular dominance stripes in the striate cortex and visual field of the macaque monkey. The Journal of neuroscience 5, 486501.

Liang, X., Connelly, A., Tournier, J.D., and Calamante, F. (2014). A variable flip angle-based method for reducing blurring in 3D GRASE ASL. Phys Med Biol 59, 5559-5573. doi: 10.1088/00319155/59/18/5559.

Lotze, M., Erb, M., Flor, H., Huelsmann, E., Godde, B., and Grodd, W. (2000). $\mathrm{fMRI}$ evaluation of somatotopic representation in human primary motor cortex. Neuroimage 11, 473-481.

Lu, H., Golay, X., Pekar, J.J., and Van Zijl, P. (2003). Functional magnetic resonance imaging based on changes in vascular space occupancy. Magnetic Resonance in Medicine 50, 263-274. 
Lustig, M., Donoho, D., and Pauly, J.M. (2007). Sparse MRI: The application of compressed sensing for rapid MR imaging. Magn Reson Med 58, 1182-1195. doi: 10.1002/mrm.21391.

Lutti, A., Dick, F., Sereno, M.I., and Weiskopf, N. (2014). Using highresolution quantitative mapping of R1 as an index of cortical myelination. Neuroimage 93 Pt 2, 176-188. doi: 10.1016/j.neuroimage.2013.06.005.

Ma, D., Gulani, V., Seiberlich, N., Liu, K., Sunshine, J.L., Duerk, J.L., and Griswold, M.A. (2013). Magnetic resonance fingerprinting. Nature 495, 187-192. doi: 10.1038/nature11971.

Malach, R., Amir, Y., Harel, M., and Grinvald, A. (1993). Relationship between intrinsic connections and functional architecture revealed by optical imaging and in vivo targeted biocytin injections in primate striate cortex. Proceedings of the National Academy of Sciences 90, 10469-10473.

Mansfield, P. (1977). Multi-planar image formation using NMR spin echoes. Journal of Physics C: Solid State Physics 10, L55. doi: 10.1088/00223719/10/3/004.

Margosian, P., Schmitt, F., and Purdy, D. (1986). Faster MR imaging: imaging with half the data. Health Care Instrum 1, 195.

Marques, J.P., Kober, T., Krueger, G., Van Der Zwaag, W., Van De Moortele, P.F., and Gruetter, R. (2010). MP2RAGE, a self bias-field corrected sequence for improved segmentation and T1-mapping at high field. Neuroimage 49, 1271-1281. doi: 10.1016/j.neuroimage.2009.10.002.

Matsubara, J., Cynader, M., and Swindale, N. (1987). Anatomical properties and physiological correlates of the intrinsic connections in cat area 18. The Journal of neuroscience 7, 1428-1446.

Mcgibney, G., Smith, M., Nichols, S., and Crawley, A. (1993). Quantitative evaluation of several partial Fourier reconstruction algorithms used in MRI. Magnetic resonance in medicine 30, 51-59.

Menon, R.S., Ogawa, S., Strupp, J.P., and Ugurbil, K. (1997). Ocular dominance in human V1 demonstrated by functional magnetic resonance imaging. Journal of Neurophysiology 77, 2780-2787.

Metzger, G.J., Snyder, C., Akgun, C., Vaughan, T., Ugurbil, K., and Van De Moortele, P.F. (2008). Local B1+ shimming for prostate imaging with transceiver arrays at 7T based on subject-dependent transmit phase measurements. Magn Reson Med 59, 396-409. doi: $10.1002 / \mathrm{mrm} .21476$.

Miller, K.L., and Jezzard, P. (2008). Modeling SSFP functional MRI contrast in the brain. Magn Reson Med 60, 661-673. doi: 10.1002/mrm.21690. 
Moeller, S., Yacoub, E., Olman, C.A., Auerbach, E., Strupp, J., Harel, N., and Ugurbil, K. (2010). Multiband multislice GE-EPI at 7 tesla, with 16fold acceleration using partial parallel imaging with application to high spatial and temporal whole-brain fMRI. Magn Reson Med 63, 1144-1153. doi: 10.1002/mrm.22361.

Mountcastle, V.B. (1957). Modality and topographic properties of single neurons of cat's somatic sensory cortex. J Neurophysiol 20, 408434.

Muckli, L., De Martino, F., Vizioli, L., Petro, L.S., Smith, F.W., Ugurbil, K., Goebel, R., and Yacoub, E. (2015). Contextual Feedback to Superficial Layers of V1. Current Biology.

Mugler, J.P., 3rd, Epstein, F.H., and Brookeman, J.R. (1992). Shaping the signal response during the approach to steady state in threedimensional magnetization-prepared rapid gradient-echo imaging using variable flip angles. Magn Reson Med 28, 165-185. doi: 10.1002/mrm.1910280202.

Mugler, J.P., and Brookeman, J.R. (1990). Three-dimensional magnetization-prepared rapid gradient-echo imaging (3D MP RAGE). Magnetic Resonance in Medicine 15, 152-157.

Murphy, K., Bodurka, J., and Bandettini, P.A. (2007). How long to scan? The relationship between $\mathrm{fMRI}$ temporal signal to noise ratio and necessary scan duration. Neurolmage 34, 565-574. doi: 10.1016/j.neuroimage.2006.09.032.

Nehrke, K., and Börnert, P. (2012). DREAM-a novel approach for robust, ultrafast, multislice B1 mapping. Magnetic Resonance in Medicine 68, 1517-1526.

Nieuwenhuys, R. (2013). "The myeloarchitectonic studies on the human cerebral cortex of the Vogt-Vogt school, and their significance for the interpretation of functional neuroimaging data," in Microstructural Parcellation of the Human Cerebral Cortex. Springer), 55-125.

Noll, D.C., Nishimura, D.G., and Macovski, A. (1991). Homodyne detection in magnetic resonance imaging. IEEE Trans Med Imaging 10, 154163. doi: $10.1109 / 42.79473$.

Norris, D.G. (2012). Spin-echo fMRI: The poor relation? Neuroimage 62, 1109-1115. doi: 10.1016/j.neuroimage.2012.01.003.

Ogawa, S., Lee, T.M., Kay, A.R., and Tank, D.W. (1990). Brain magnetic resonance imaging with contrast dependent on blood oxygenation. Proc Natl Acad Sci U S A 87, 9868-9872.

Ogawa, S., Tank, D.W., Menon, R., Ellermann, J.M., Kim, S.G., Merkle, H., and Ugurbil, K. (1992). Intrinsic signal changes accompanying 
sensory stimulation: functional brain mapping with magnetic resonance imaging. Proc Natl Acad Sci U S A 89, 5951-5955.

Olman, C.A., Harel, N., Feinberg, D.A., He, S., Zhang, P., Ugurbil, K., and Yacoub, E. (2012). Layer-specific fMRI reflects different neuronal computations at different depths in human V1. PLoS One 7, e32536. doi: 10.1371/journal.pone.0032536.

Olman, C.A., Inati, S., and Heeger, D.J. (2007). The effect of large veins on spatial localization with GE BOLD at $3 \mathrm{~T}$ : Displacement, not blurring. Neuroimage 34, 1126-1135.

Oshio, K., and Feinberg, D.A. (1991). GRASE (Gradient- and spin-echo) imaging: a novel fast MRI technique. Magn Reson Med 20, 344349. doi: 10.1148/radiology.181.2.1924811.

Panchuelo, R.M.S., Schluppeck, D., Harmer, J., Bowtell, R., and Francis, S. (2015). Assessing the Spatial Precision of SE and GE-BOLD Contrast at 7 Tesla. Brain topography 28, 62-65.

Park, H.W., Kim, D.J., and Cho, Z.H. (1987). Gradient reversal technique and its applications to chemical-shift-related NMR imaging. Magn Reson Med 4, 526-536. doi: 10.1002/mrm.1910040604.

Park, J., Mugler, J.P., 3rd, Horger, W., and Kiefer, B. (2007). Optimized T1weighted contrast for single-slab 3D turbo spin-echo imaging with long echo trains: application to whole-brain imaging. Magn Reson Med 58, 982-992. doi: 10.1002/mrm.21386.

Park, J., Ronen, I., Kim, D., and Ugurbil, K. (2005). Spatial specificity of high resolution gradient echo (GE) BOLD and spin echo (SE) BOLD fMRI in cat visual cortex at 9.4 T. 13th Annual Meeting of ISMRM 31.

Parkes, L.M., Schwarzbach, J.V., Bouts, A.A., Deckers, R.H., Pullens, P., Kerskens, C.M., and Norris, D.G. (2005). Quantifying the spatial resolution of the gradient echo and spin echo BOLD response at 3 Tesla. Magn Reson Med 54, 1465-1472. doi: 10.1002/mrm.20712.

Pauly, J., Nishimura, D., and Macovski, A. (1989). A k-space analysis of small-tip-angle excitation. Journal of Magnetic Resonance (1969) 81, 43-56. doi: 10.1016/j.jmr.2011.09.023.

Peters, A.M., Brookes, M.J., Hoogenraad, F.G., Gowland, P.A., Francis, S.T., Morris, P.G., and Bowtell, R. (2007). T2* measurements in human brain at 1.5, 3 and 7 T. Magn Reson Imaging 25, 748-753. doi: 10.1016/j.mri.2007.02.014.

Pfeuffer, J., Van De Moortele, P.-F., Yacoub, E., Shmuel, A., Adriany, G., Andersen, P., Merkle, H., Garwood, M., Ugurbil, K., and Hu, X. (2002). Zoomed Functional Imaging in the Human Brain at 7 Tesla with Simultaneous High Spatial and High Temporal Resolution. Neurolmage 17, 272-286. doi: 10.1006/nimg.2002.1103. 
Pflugfelder, D., Vahedipour, K., Uludag, K., Shah, N.J., and Stocker, T. (2011). On the numerically predicted spatial BOLD fMRI specificity for spin echo sequences. Magn Reson Imaging 29, 1195-1204. doi: 10.1016/j.mri.2011.07.015.

Pohmann, R., Speck, O., and Scheffler, K. (2015). Signal-to-noise ratio and MR tissue parameters in human brain imaging at 3, 7, and 9.4 tesla using current receive coil arrays. Magn Reson Med. doi: 10.1002/mrm.25677.

Polimeni, J.R., Bianciardi, M., Keil, B., and Wald, L.L. (2015). Cortical depth dependence of physiological fluctuations and whole-brain restingstate functional connectivity at 7T. 23nd Annual Meeting of ISMRM 23, 592.

Polimeni, J.R., Fischl, B., Greve, D.N., and Wald, L.L. (2010). Laminar analysis of 7T BOLD using an imposed spatial activation pattern in human V1. Neuroimage 52, 1334-1346. doi: 10.1016/j.neuroimage.2010.05.005.

Poser, B.A., Anderson, R.J., Guérin, B., Setsompop, K., Deng, W., Mareyam, A., Serano, P., Wald, L.L., and Stenger, V.A. (2014). Simultaneous multislice excitation by parallel transmission. Magnetic Resonance in Medicine 71, 1416-1427.

Poser, B.A., Koopmans, P.J., Witzel, T., Wald, L.L., and Barth, M. (2010). Three dimensional echo-planar imaging at 7 Tesla. Neuroimage 51, 261-266. doi: 10.1016/j.neuroimage.2010.01.108.

Poser, B.A., and Norris, D.G. (2007). Fast spin echo sequences for BOLD functional MRI. MAGMA 20, 11-17. doi: 10.1007/s10334-006-0063$x$.

Pruessmann, K.P., Weiger, M., Scheidegger, M.B., and Boesiger, P. (1999). SENSE: sensitivity encoding for fast MRI. Magnetic resonance in medicine 42, 952-962.

Qin, Q. (2012). Point spread functions of the T2 decay in k-space trajectories with long echo train. Magn Reson Imaging 30, 11341142. doi: 10.1016/j.mri.2012.04.017.

Ramón Y Cajal, S. (1899). Comparative study of the sensory areas of the human cortex.

Reichenbach, J.R., Venkatesan, R., Yablonskiy, D.A., Thompson, M.R., Lai, S., and Haacke, E.M. (1997). Theory and application of static field inhomogeneity effects in gradient-echo imaging. Journal of Magnetic Resonance Imaging 7, 266-279. doi: 10.1002/jmri.1880070203.

Renvall, V., Witzel, T., Wald, L.L., and Polimeni, J.R. (2014). Fast variable inversion-recovery time EPI for anatomical reference and quantitative T1 mapping. 22nd Annual Meeting of ISMRM 22, 4282. 
Ress, D., Glover, G.H., Liu, J., and Wandell, B. (2007). Laminar profiles of functional activity in the human brain. Neurolmage 34, 74-84. doi: 10.1016/j.neuroimage.2006.08.020.

Robson, M.D., Gore, J.C., and Constable, R.T. (1997). Measurement of the point spread function in MRI using constant time imaging. Magn Reson Med 38, 733-740. doi: 10.1002/mrm.1910380509.

Roemer, P., Edelstein, W., Hayes, C., Souza, S., and Mueller, O. (1990). The NMR phased array. Magnetic resonance in medicine 16, 192-225.

Roland, E., and Larsen, B. (1976). Focal increase of cerebral blood flow during stereognostic testing in man. Arch Neurol 33, 551-558.

Roland, P.E., Skinhoj, E., Lassen, N.A., and Larsen, B. (1980). Different cortical areas in man in organization of voluntary movements in extrapersonal space. J Neurophysiol 43, 137-150.

Rossmann, K. (1969). Point Spread-Function, Line Spread-Function, and Modulation Transfer Function: Tools for the Study of Imaging Systems 1. Radiology 93, 257-272.

Roy, C.S., and Sherrington, C. (1890). On the regulation of the blood-supply of the brain. The Journal of physiology 11, 85.

Sánchez-Panchuelo, R.M., Francis, S.T., Schluppeck, D., and Bowtell, R.W. (2012). Correspondence of human visual areas identified using functional and anatomical MRI in vivo at $7 \mathrm{~T}$. Journal of Magnetic Resonance Imaging 35, 287-299.

Scheffler, K., and Ehses, P. (2015). High-resolution mapping of neuronal activation with balanced SSFP at 9.4 tesla. Magnetic resonance in medicine.

Schira, M.M., Tyler, C.W., Breakspear, M., and Spehar, B. (2009). The foveal confluence in human visual cortex. The Journal of Neuroscience 29, 9050-9058.

Senden, M., Reithler, J., Gijsen, S., and Goebel, R. (2014). Evaluating population receptive field estimation frameworks in terms of robustness and reproducibility. PLoS One 9, e114054. doi: 10.1371/journal.pone.0114054.

Setsompop, K., Gagoski, B.A., Polimeni, J.R., Witzel, T., Wedeen, V.J., and Wald, L.L. (2012). Blipped-controlled aliasing in parallel imaging for simultaneous multislice echo planar imaging with reduced g-factor penalty. Magn Reson Med 67, 1210-1224. doi: 10.1002/mrm.23097.

Setsompop, K., Kimmlingen, R., Eberlein, E., Witzel, T., Cohen-Adad, J., Mcnab, J.A., Keil, B., Tisdall, M.D., Hoecht, P., and Dietz, P. (2013). Pushing the limits of in vivo diffusion MRI for the Human Connectome Project. Neuroimage 80, 220-233. 
Shapley, R., and Hawken, M.J. (2011). Color in the cortex: single-and double-opponent cells. Vision research 51, 701-717.

Shmuel, A., Chaimow, D., Raddatz, G., Ugurbil, K., and Yacoub, E. (2010). Mechanisms underlying decoding at $7 \mathrm{~T}$ : ocular dominance columns, broad structures, and macroscopic blood vessels in V1 convey information on the stimulated eye. Neuroimage 49, 19571964. doi: 10.1016/j.neuroimage.2009.08.040.

Shmuel, A., Yacoub, E., Chaimow, D., Logothetis, N.K., and Ugurbil, K. (2007). Spatio-temporal point-spread function of $\mathrm{fMRI}$ signal in human gray matter at 7 Tesla. Neuroimage 35, 539-552. doi: 10.1016/j.neuroimage.2006.12.030.

Shoham, D., Hübener, M., Schulze, S., Grinvald, A., and Bonhoeffer, T. (1997). Spatio-temporal frequency domains and their relation to cytochrome oxidase staining in cat visual cortex. Nature 385, 529533.

Shrivastava, D., Hanson, T., Schlentz, R., Gallaghar, W., Snyder, C., Delabarre, L., Prakash, S., laizzo, P., and Vaughan, J.T. (2008). Radiofrequency heating at 9.4T: in vivo temperature measurement results in swine. Magn Reson Med 59, 73-78. doi: $10.1002 / \mathrm{mrm} .21425$.

Siero, J.C., Hendrikse, J., Hoogduin, H., Petridou, N., Luijten, P., and Donahue, M.J. (2015). Cortical depth dependence of the BOLD initial dip and poststimulus undershoot in human visual cortex at 7 Tesla. Magn Reson Med 73, 2283-2295. doi: 10.1002/mrm.25349.

Silver, M.A., and Kastner, S. (2009). Topographic maps in human frontal and parietal cortex. Trends Cogn Sci 13, 488-495. doi: 10.1016/j.tics.2009.08.005.

Sincich, L.C., and Horton, J.C. (2005). The circuitry of V1 and V2: integration of color, form, and motion. Annu. Rev. Neurosci. 28, 303-326.

Smith, F.W., and Muckli, L. (2010). Nonstimulated early visual areas carry information about surrounding context. Proc Natl Acad Sci U S A 107, 20099-20103. doi: 10.1073/pnas.1000233107.

Smith, S.M., Jenkinson, M., Woolrich, M.W., Beckmann, C.F., Behrens, T.E., Johansen-Berg, H., Bannister, P.R., De Luca, M., Drobnjak, I., Flitney, D.E., Niazy, R.K., Saunders, J., Vickers, J., Zhang, Y., De Stefano, N., Brady, J.M., and Matthews, P.M. (2004). Advances in functional and structural MR image analysis and implementation as FSL. Neuroimage 23 Suppl 1, S208-219. doi: 10.1016/j.neuroimage.2004.07.051.

Sodickson, D.K., and Manning, W.J. (1997). Simultaneous acquisition of spatial harmonics (SMASH): fast imaging with radiofrequency coil arrays. Magnetic Resonance in Medicine 38, 591-603. 
Sotiropoulos, S.N., Moeller, S., Jbabdi, S., Xu, J., Andersson, J.L., Auerbach, E.J., Yacoub, E., Feinberg, D., Setsompop, K., Wald, L.L., Behrens, T.E., Ugurbil, K., and Lenglet, C. (2013). Effects of image reconstruction on fiber orientation mapping from multichannel diffusion MRI: reducing the noise floor using SENSE. Magn Reson Med 70, 1682-1689. doi: 10.1002/mrm.24623.

Stöcker, T., and Shah, N.J. (2006). MP-SAGE: A new MP-RAGE sequence with enhanced SNR and CNR for brain imaging utilizing squarespiral phase encoding and variable flip angles. Magnetic resonance in medicine $56,824-834$.

Sun, P., Ueno, K., Waggoner, R.A., Gardner, J.L., Tanaka, K., and Cheng, K. (2007). A temporal frequency-dependent functional architecture in human V1 revealed by high-resolution fMRI. Nat Neurosci 10, 1404-1406. doi: 10.1038/nn1983.

Trampel, R., Reimer, E., Huber, L., Ivanov, D., Heidemann, R.M., Schafer, A., and Turner, R. (2013). Anatomical brain imaging at 7T using twodimensional GRASE. Magn Reson Med. doi: 10.1002/mrm.25047.

Triantafyllou, C., Hoge, R.D., Krueger, G., Wiggins, C.J., Potthast, A., Wiggins, G.C., and Wald, L.L. (2005). Comparison of physiological noise at $1.5 \mathrm{~T}, 3 \mathrm{~T}$ and $7 \mathrm{~T}$ and optimization of $\mathrm{fMRI}$ acquisition parameters. Neuroimage 26, 243-250. doi: 10.1016/j.neuroimage.2005.01.007.

Triantafyllou, C., Polimeni, J.R., Elschot, M., and Wald, L.L. (2009). Physiological Noise in Gradient Echo and Spin Echo EPI at 3T and 7T. 17th Annual Meeting International Society for Magnetic Resonance in Medicine, 122.

Triantafyllou, C., Polimeni, J.R., and Wald, L.L. (2011). Physiological noise and signal-to-noise ratio in $\mathrm{fMRI}$ with multi-channel array coils. Neuroimage 55, 597-606. doi: 10.1016/j.neuroimage.2010.11.084.

Ts'o, D.Y., Frostig, R.D., Lieke, E.E., and Grinvald, A. (1990). Functional organization of primate visual cortex revealed by high resolution optical imaging. Science 249, 417-420.

Ts'o, D.Y., Zarella, M., and Burkitt, G. (2009). Whither the hypercolumn? J Physiol 587, 2791-2805. doi: 10.1113/jphysiol.2009.171082.

Tse, D.H., Poole, M.S., Magill, A.W., Felder, J., Brenner, D., and Jon Shah, N. (2014). Encoding methods for B1(+) mapping in parallel transmit systems at ultra high field. J Magn Reson 245, 125-132. doi: 10.1016/j.jmr.2014.06.006.

Turner, R., Jezzard, P., Wen, H., Kwong, K.K., Le Bihan, D., Zeffiro, T., and Balaban, R.S. (1993). Functional mapping of the human visual cortex at 4 and 1.5 tesla using deoxygenation contrast EPI. Magn Reson Med 29, 277-279. 
Twieg, D.B. (1983). The k-trajectory formulation of the NMR imaging process with applications in analysis and synthesis of imaging methods. Medical Physics 10, 610-621.

Uğurbil, K., Adriany, G., Andersen, P., Chen, W., Garwood, M., Gruetter, R., Henry, P.-G., Kim, S.-G., Lieu, H., and Tkac, I. (2003). Ultrahigh field magnetic resonance imaging and spectroscopy. Magnetic resonance imaging 21, 1263-1281.

Ugurbil, K., Xu, J., Auerbach, E.J., Moeller, S., Vu, A.T., Duarte-Carvajalino, J.M., Lenglet, C., Wu, X., Schmitter, S., Van De Moortele, P.F., Strupp, J., Sapiro, G., De Martino, F., Wang, D., Harel, N., Garwood, M., Chen, L., Feinberg, D.A., Smith, S.M., Miller, K.L., Sotiropoulos, S.N., Jbabdi, S., Andersson, J.L., Behrens, T.E., Glasser, M.F., Van Essen, D.C., and Yacoub, E. (2013). Pushing spatial and temporal resolution for functional and diffusion $M R I$ in the Human Connectome Project. Neuroimage 80, 80-104. doi: 10.1016/j.neuroimage.2013.05.012.

Uludag, K. (2010). To dip or not to dip: reconciling optical imaging and fMRI data. Proc Natl Acad Sci U S A 107, E23; author reply E24. doi: 10.1073/pnas.0914194107.

Uludag, K., Dubowitz, D.J., and Buxton, R.B. (2005). "Principles of functional imaging of the brain.," in Clinical Magnetic Resonance Imaging (3rd $E d)$, eds. R.R. Edelmann, J.R. Hesselink \& M.B. Zlatkin. (England).

Uludag, K., Muller-Bierl, B., and Ugurbil, K. (2009). An integrative model for neuronal activity-induced signal changes for gradient and spin echo functional imaging. Neuroimage 48, 150-165. doi: 10.1016/j.neuroimage.2009.05.051.

Van De Moortele, P.F., Auerbach, E.J., Olman, C., Yacoub, E., Ugurbil, K., and Moeller, S. (2009). T1 weighted brain images at 7 Tesla unbiased for Proton Density, T2* contrast and RF coil receive B1 sensitivity with simultaneous vessel visualization. Neuroimage 46, 432-446. doi: 10.1016/j.neuroimage.2009.02.009.

Van Der Kouwe, A.J., Benner, T., Salat, D.H., and Fischl, B. (2008). Brain morphometry with multiecho MPRAGE. Neuroimage 40, 559-569. doi: 10.1016/j.neuroimage.2007.12.025.

Van Der Kouwe, A.J., Tisdall, M.D., Bhat, H., Fischl, B., and Polimeni, J.R. (2014). Multiple Echo and Inversion Time MPRAGE with Inner Loop GRAPPA Acceleration and Prospective Motion Correction for Minimally Distorted Multispectral Brain Morphometry. 22nd Annual Meeting of ISMRM 22, 120.

Vogt, C., and Vogt, O. (1919). Allgemeine ergebnisse unserer hirnforschung. JA Barth. 
Volk, A., Tiffon, B., Mispelter, J., and Lhoste, J.-M. (1987). Chemical shiftspecific slice selection. A new method for chemical shift imaging at high magnetic field. Journal of Magnetic Resonance (1969) 71, 168174. doi: 10.1016/0022-2364(87)90141-7.

Vu, A., Feinberg, D., Harel, N., Ugurbil, K., and Yacoub, E. (2013). Diagonal multi-slab inner volume 3D GRASE imaging for high resolution T2 weighted fMRI. Proceedings of the 21st Annual Meeting of ISMRM 21, 2364.

Waehnert, M.D., Dinse, J., Weiss, M., Streicher, M.N., Waehnert, P., Geyer, S., Turner, R., and Bazin, P.L. (2014). Anatomically motivated modeling of cortical laminae. Neuroimage 93 Pt 2, 210-220. doi: 10.1016/j.neuroimage.2013.03.078.

Wandell, B.A., Dumoulin, S.O., and Brewer, A.A. (2007). Visual field maps in human cortex. Neuron 56, 366-383.

Wandell, B.A., and Winawer, J. (2011). Imaging retinotopic maps in the human brain. Vision Res 51, 718-737. doi: 10.1016/j.visres.2010.08.004.

Wargo, C.J., and Gore, J.C. (2013). Localized high-resolution DTI of the human midbrain using single-shot EPI, parallel imaging, and outervolume suppression at $7 \mathrm{~T}$. Magnetic Resonance Imaging 31, 810819. doi: 10.1016/j.mri.2013.01.013.

Watanabe, M., Bartels, A., Macke, J.H., Murayama, Y., and Logothetis, N.K. (2013). Temporal jitter of the BOLD signal reveals a reliable initial dip and improved spatial resolution. Curr Biol 23, 2146-2150. doi: 10.1016/j.cub.2013.08.057.

Weiskopf, N., Hutton, C., Josephs, O., and Deichmann, R. (2006). Optimal EPI parameters for reduction of susceptibility-induced BOLD sensitivity losses: a whole-brain analysis at $3 \mathrm{~T}$ and $1.5 \mathrm{~T}$. Neuroimage 33, 493-504. doi: 10.1016/j.neuroimage.2006.07.029.

Weiskopf, N., Hutton, C., Josephs, O., Turner, R., and Deichmann, R. (2007). Optimized EPI for $\mathrm{FMRI}$ studies of the orbitofrontal cortex: compensation of susceptibility-induced gradients in the readout direction. Magnetic Resonance Materials in Physics, Biology and Medicine 20, 39-49.

Weliky, M., Bosking, W.H., and Fitzpatrick, D. (1996). A systematic map of direction preference in primary visual cortex.

Wernicke, C. (1874). Der aphasische Symptomencomplex: eine psychologische Studie auf anatomischer Basis. Cohn.

Wiesel, T., Hubel, D., and Lam, D. (1974). Autoradiographic demonstration of ocular-dominance columns in the monkey striate cortex by means of transneuronal transport. Brain research 79, 273-279. 
Wiggins, G.C., Polimeni, J.R., Potthast, A., Schmitt, M., Alagappan, V., and Wald, L.L. (2009). 96-Channel receive-only head coil for 3 Tesla: design optimization and evaluation. Magn Reson Med 62, 754-762. doi: 10.1002/mrm.22028.

Wilms, M., Eickhoff, S.B., Homke, L., Rottschy, C., Kujovic, M., Amunts, K., and Fink, G.R. (2010). Comparison of functional and cytoarchitectonic maps of human visual areas V1, V2, V3d, V3v, and V4(v). Neuroimage 49, 1171-1179. doi: 10.1016/j.neuroimage.2009.09.063.

Winawer, J., Horiguchi, H., Sayres, R.A., Amano, K., and Wandell, B.A. (2010). Mapping hV4 and ventral occipital cortex: the venous eclipse. Journal of Vision 10, 1.

Wohlschläger, A.M., Specht, K., Lie, C., Mohlberg, H., Wohlschläger, A., Bente, K., Pietrzyk, U., Stöcker, T., Zilles, K., and Amunts, K. (2005). Linking retinotopic $\mathrm{fMRI}$ mapping and anatomical probability maps of human occipital areas V1 and V2. Neuroimage 26, 73-82.

Wu, X., Schmitter, S., Auerbach, E.J., Moeller, S., Uğurbil, K., and De Moortele, V. (2013). Simultaneous multislice multiband parallel radiofrequency excitation with independent slice-specific transmit B1 homogenization. Magnetic Resonance in Medicine 70, 630-638.

$\mathrm{Xu}$, J., Moeller, S., Auerbach, E.J., Strupp, J., Smith, S.M., Feinberg, D.A., Yacoub, E., and Ugurbil, K. (2013). Evaluation of slice accelerations using multiband echo planar imaging at 3 T. Neuroimage 83, 9911001. doi: 10.1016/j.neuroimage.2013.07.055.

Yacoub, E., Duong, T.Q., Van De Moortele, P.F., Lindquist, M., Adriany, G., Kim, S.G., Ugurbil, K., and Hu, X. (2003). Spin-echo fMRI in humans using high spatial resolutions and high magnetic fields. Magn Reson Med 49, 655-664. doi: 10.1002/mrm.10433.

Yacoub, E., Harel, N., and Ugurbil, K. (2008). High-field fMRI unveils orientation columns in humans. Proc Natl Acad Sci U S A 105, 10607-10612. doi: 10.1073/pnas.0804110105.

Yacoub, E., Shmuel, A., Logothetis, N., and Ugurbil, K. (2007). Robust detection of ocular dominance columns in humans using Hahn Spin Echo BOLD functional MRI at 7 Tesla. Neuroimage 37, 1161-1177. doi: 10.1016/j.neuroimage.2007.05.020.

Yacoub, E., Shmuel, A., Pfeuffer, J., Van De Moortele, P.F., Adriany, G., Ugurbil, K., and $\mathrm{Hu}, \mathrm{X}$. (2001). Investigation of the initial dip in $\mathrm{fMRI}$ at 7 Tesla. NMR Biomed 14, 408-412.

Yacoub, E., Ugurbil, K., and Harel, N. (2006). The spatial dependence of the poststimulus undershoot as revealed by high-resolution BOLD- and CBV-weighted fMRI. J Cereb Blood Flow Metab 26, 634-644. doi: 10.1038/sj.jcbfm.9600239. 
Yacoub, E., Van De Moortele, P.F., Shmuel, A., and Ugurbil, K. (2005). Signal and noise characteristics of Hahn SE and GE BOLD fMRI at $7 \mathrm{~T}$ in humans. Neuroimage 24, 738-750. doi: 10.1016/j.neuroimage.2004.09.002.

Yoshioka, T., Blasdel, G.G., Levitt, J.B., and Lund, J.S. (1996). Relation between patterns of intrinsic lateral connectivity, ocular dominance, and cytochrome oxidase-reactive regions in macaque monkey striate cortex. Cerebral Cortex 6, 297-310.

Young, I., Cox, I., Bryant, D., and Bydder, G. (1988). The benefits of increasing spatial resolution as a means of reducing artifacts due to field inhomogeneities. Magnetic resonance imaging 6, 585-590.

Zaitsev, M., Dold, C., Sakas, G., Hennig, J., and Speck, O. (2006). Magnetic resonance imaging of freely moving objects: prospective real-time motion correction using an external optical motion tracking system. Neuroimage 31, 1038-1050.

Zaitsev, M., Hennig, J., and Speck, O. (2004). Point spread function mapping with parallel imaging techniques and high acceleration factors: fast, robust, and flexible method for echo-planar imaging distortion correction. Magn Reson Med 52, 1156-1166. doi: 10.1002/mrm.20261.

Zappe, A.C., Pfeuffer, J., Merkle, H., Logothetis, N.K., and Goense, J.B. (2008). The effect of labeling parameters on perfusion-based fMRI in nonhuman primates. Journal of Cerebral Blood Flow \& Metabolism 28, 640-652.

Zeng, H., and Constable, R.T. (2002). Image distortion correction in EPI: comparison of field mapping with point spread function mapping. Magn Reson Med 48, 137-146. doi: 10.1002/mrm.10200.

Zhang, W.T., Mainero, C., Kumar, A., Wiggins, C.J., Benner, T., Purdon, P.L., Bolar, D.S., Kwong, K.K., and Sorensen, A.G. (2006). Strategies for improving the detection of $\mathrm{FMRI}$ activation in trigeminal pathways with cardiac gating. Neuroimage 31, 1506-1512. doi: 10.1016/j.neuroimage.2006.02.033.

Zhao, F., Wang, P., and Kim, S.G. (2004). Cortical depth-dependent gradient-echo and spin-echo BOLD fMRI at $9.4 \mathrm{~T}$. Magnetic resonance in medicine 51, 518-524.

Zhu, Y. (2004). Parallel excitation with an array of transmit coils. Magnetic Resonance in Medicine 51, 775-784.

Zilles, K., and Amunts, K. (2009). Receptor mapping: architecture of the human cerebral cortex. Current opinion in neurology 22, 331-339.

Zimmermann, J., Goebel, R., De Martino, F., Van De Moortele, P.F., Feinberg, D., Adriany, G., Chaimow, D., Shmuel, A., Ugurbil, K., and Yacoub, E. (2011). Mapping the organization of axis of motion 
selective features in human area MT using high-field fMRI. PLoS One 6, e28716. doi: 10.1371/journal.pone.0028716. 
Notes 
Notes 
Notes 
Notes

201 
Notes

202 
Notes 
Notes 
Notes

205 
Notes 
Notes 
Notes

208 